Supporting information

for the article:

\title{
Pd-NHC Catalytic System for the Efficient Atom-Economic Synthesis of Vinyl Sulfides from Tertiary, Secondary or Primary Thiols
}

Evgeniya S. Degtyareva, ${ }^{a}$ Julia V. Burykina, ${ }^{\text {a }}$ Artem N. Fakhrutdinov, ${ }^{a}$ Evgeniy G. Gordeev, ${ }^{a}$ Victor N. Khrustalev ${ }^{b}$ and Valentine P. Ananikov ${ }^{* a, c}$

${ }^{a}$ Zelinsky Institute of Organic Chemistry, Russian Academy of Sciences, Leninsky Prospect 47, Moscow, 119991, Russia

${ }^{\mathrm{b}}$ Nesmeyanov Institute of Organoelement Compounds, Russian Academy of Sciences, Vavilov str., 28, Moscow, 119991, Russia

${ }^{\mathrm{C}}$ Department of Chemistry, Saint Petersburg State University, Stary Petergof, 198504, Russia

*e-mail: val@ioc.ac.ru 


\section{Tables of Contents:}

S1. General Information 3

S2. Catalyst Screening $\quad 4$

S3. Additional Data on Reaction Scope 6

S4. Mechanistic Studies 6

$\begin{array}{ll}\text { S5. Kinetic Measurements } & 10\end{array}$

S6. Deuterium Labeling Studies 12

S7. Theoretical Calculations of the Key Alkyne Insertion Stage 12

S8. Experimental Procedure 16

S9. X-Ray Crystal Structure Determination of Ila 27

S10. References 36

$\begin{array}{ll}\text { S11. NMR Spectra } & 37\end{array}$ 


\section{S1. General Information}

The reagents were obtained from commercial sources and used as supplied (verified by NMR prior to use). The solvents were purified according to published methods. The solvents for NMR spectroscopy were obtained from Deutero $\mathrm{GmbH}$. Acetonitrile (HPLC-grade) for ESI-MS was obtained from Merck and used as supplied. Unless otherwise noted, the reactions were carried out in PTFE screw-capped tubes that were equipped with a magnetic stirrer bar.

NMR measurements were performed using Avance-400, Bruker DRX-500 and Avance-600 spectrometers operating at $400.1 / 500.1 / 600.1$ and $101 / 125.8 / 150.9 \mathrm{MHz}$ for ${ }^{1} \mathrm{H}$ and ${ }^{13} \mathrm{C}$ nuclei, respectively. The spectra were processed on a Linux workstation using the TopSpin software package. The ${ }^{1} \mathrm{H}$ and ${ }^{13} \mathrm{C}$ chemical shifts are reported relative to the corresponding solvent signals, which were used as internal references. The yields given below were calculated based on the initial thiol amount.

The high-resolution mass spectra (HRMS) were recorded on a Bruker microTOF instrument equipped with an electrospray ionization ion source (ESI). The measurements were performed in + MS mode with an interface capillary voltage of $4500 \mathrm{~V}$ and a scanned range of $m / z$ 50-3000. In addition, external calibration of the instrument was performed with electrospray calibrant solution (Fluka). A direct syringe infusion was used for the acetonitrile solutions (flow rate $3 \mu \mathrm{L} / \mathrm{min}$ ). Nitrogen was applied as a dry gas, and the interface temperature was set to $180^{\circ} \mathrm{C}$. Unless otherwise noted, the samples (acetonitrile solutions) for ESI-HRMS were prepared in $1.5 \mathrm{~mL}$ Eppendorf tubes. The spectra were processed using the Bruker DataAnalysis 4.0 software package. 


\section{S2. Catalyst Screening}

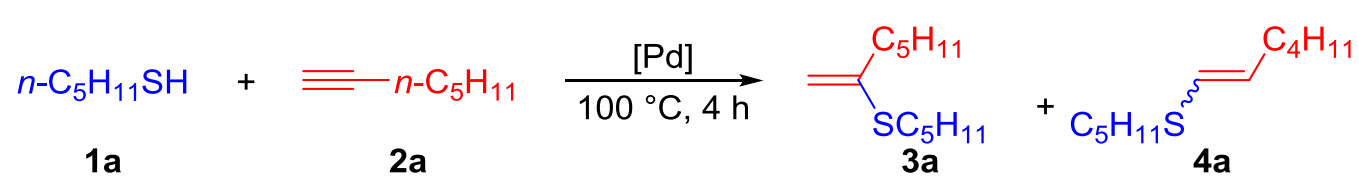

The reaction of 1-pentanethiol (1a) with 1-heptyne (2a) (eq. S1) was chosen as a model for catalyst screening (Table S1, S2). The $\mathrm{Ni}(\mathrm{acac})_{2}, \mathrm{Ni}\left(\mathrm{P}(\mathrm{OPh})_{3}\right)_{4}, \mathrm{Ni}\left(\mathrm{PPh}_{3}\right)_{2} \mathrm{Cl}_{2}$, $\mathrm{Pd}(\mathrm{OAc})_{2}, \mathrm{Pt}\left(\mathrm{PPh}_{3}\right)_{4}, \mathrm{Cu}(\mathrm{acac})_{2}, \mathrm{Cu}\left(\mathrm{PPh}_{3}\right) \mathrm{Br}, \mathrm{Fe}(\mathrm{acac})_{3}, \mathrm{Co}(\mathrm{acac})_{2}, \mathrm{Co}\left(\mathrm{PPh}_{3}\right)_{3} \mathrm{Cl}$, and $\mathrm{Co}\left(\mathrm{PPh}_{3}\right)_{2} \mathrm{Cl}_{2}$ complexes were tested, and no product formation was observed. $\mathrm{Pd}(\mathrm{OAc})_{2}$ was further tested in the model reaction with variable ligands (Table $\mathrm{S1}$ ). $\mathrm{Pd}(\mathrm{OAc})_{2}$ $(2 \mathrm{~mol} \%, 0.0013 \mathrm{~g})$ was placed in a screw-capped tube followed by $8 \mathrm{~mol} \%$ of ligand. Then, 1-heptyne $\left(0.3 \cdot 10^{-3} \mathrm{~mol}, 0.0289 \mathrm{~g}\right)$ and 1 -pentanethiol $\left(0.3 \cdot 10^{-3} \mathrm{~mol}, 0.0313 \mathrm{~g}\right)$ were added. The tube was flushed with Ar and closed. The reaction was carried out for $4 \mathrm{~h}$ at $100{ }^{\circ} \mathrm{C}$. The product yield was estimated by NMR.

Table S1. Evaluation of Ligands in the Model Reaction (eq. S1) with $\operatorname{Pd}(\mathrm{OAc})_{2}$.

\begin{tabular}{|c|c|c|c|}
\hline Ligand & $\begin{array}{l}{ }^{1} \mathrm{H} \text { NMR yield } \\
\text { of } 3 a / 4 a, \%\end{array}$ & Ligand & $\begin{array}{l}{ }^{7} \mathrm{H} \text { NMR yield of } \\
3 \mathrm{a} / 4 \mathrm{a}, \%\end{array}$ \\
\hline $\begin{array}{l}\text { Tris-(m-methoxyphenyl) } \\
\text { phosphine }\end{array}$ & N.R. & Tri(m-tolyl)phosphine & $<1$ \\
\hline $\begin{array}{l}\text { Tris-(p-methoxyphenyl) } \\
\text { phosphine }\end{array}$ & $<1$ & Trimesytulphosphine & $<1$ \\
\hline Tri-o-tolylphosphine & N.R. & Diallylphenylphosphine & 3 \\
\hline Tricyclohexylphosphine & 4 & $\begin{array}{l}\text { Tert- } \\
\text { butyldichlorophosphine }\end{array}$ & N.R. \\
\hline $\begin{array}{l}\text { Dimethylphenyl- } \\
\text { phosphine }\end{array}$ & $<1$ & Tri-(2-furyl)phosphine & N.R. \\
\hline Ethyldiphenylphosphine & N.R. & $\begin{array}{l}\text { Methyldiphenyl } \\
\text { phosphine }\end{array}$ & 5 \\
\hline $\begin{array}{l}\text { Tris-(p-chlorophenyl) } \\
\text { phosphine }\end{array}$ & N.R. & Tributyl phosphite & 10 \\
\hline Triisopropyl phosphite & N.R. & $\begin{array}{l}\text { Bis(diphenylphosphino) } \\
\text { methane }\end{array}$ & 4 \\
\hline $\begin{array}{l}\text { 1,2- } \\
\text { Bis(diphenylphosphino)- } \\
\text { ethane }\end{array}$ & N.R. & Triphenyl phosphite & N.R. \\
\hline
\end{tabular}

The performance of various NHC ligands was investigated using the same model reaction (Table S2). Pd-NHC (1 mol \%) with $\mathrm{Et}_{3} \mathrm{~N}(4 \mathrm{~mol} \%, 0.0012 \mathrm{~g})$ was dissolved in $0.2 \mathrm{~mL}$ of benzene followed by addition of 1-heptyne $\left(0.3 \cdot 10^{-3} \mathrm{~mol}, 0.0289 \mathrm{~g}\right)$ and 1 pentanethiol $\left(0.3 \cdot 10^{-3} \mathrm{~mol}, 0.0313 \mathrm{~g}\right)$. The tube was flushed with $\mathrm{Ar}$ and closed. The reaction mixture was heated for 4 or $18 \mathrm{~h}$ at $100{ }^{\circ} \mathrm{C}$. 
Table S2. Evaluation of the Activity of Pd-NHC Complexes in the Model Reaction.

\begin{tabular}{|c|c|c|c|}
\hline \multirow[t]{2}{*}{ Catalyst } & & \multicolumn{2}{|c|}{ H NMR yield of $3 a / 4 a, \%$} \\
\hline & & $4 \mathrm{~h}$ & $18 h^{\mathrm{a}}$ \\
\hline \multirow{2}{*}{${ }_{R^{-N}}^{\sqrt{-}} Y^{N-R}$} & $\mathrm{R}=2,4,6$-trimethylphenyl & $4 / 12$ & $38 / 0$ \\
\hline & $\mathrm{R}=$ 2,6-diisopropylphenyl & $19 / 18$ & $41 / 18$ \\
\hline \multirow{2}{*}{$\begin{array}{l}{ }^{R-N} Y^{N-R} \\
\mathrm{Pd}^{\mathrm{N}} \mathrm{Cl}\end{array}$} & $R=2,4,6$-trimethylphenyl & $18 / 8$ & $22 / 0$ \\
\hline & $\mathrm{R}=2,6$-diisopropylphenyl & $18 / 20$ & 29/11 \\
\hline \multirow{2}{*}{$\pi_{1}^{N^{-R}}$} & $\mathrm{R}=2,4,6$-trimethylphenyl & $65 / 8$ & $77 / 0$ \\
\hline & $\mathrm{R}=$ 2,6-diisopropylphenyl & 20/11 & $35 / 20$ \\
\hline \multirow{2}{*}{$\mathrm{R}^{-\mathrm{N}} \mathrm{Y}^{\mathrm{N}} \mathrm{R}$} & $\mathrm{R}=2,4,6$-trimethylphenyl & $23 / 7$ & $31 / 0$ \\
\hline & $\mathrm{R}=$ 2,6-diisopropylphenyl & $7 / 32$ & $17 / 4$ \\
\hline \multirow{2}{*}{ 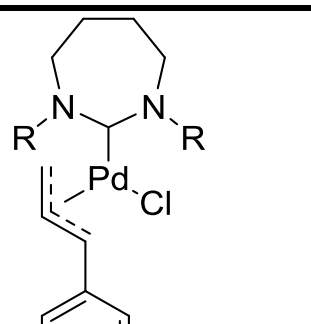 } & $\mathrm{R}=2,4,6$-trimethylphenyl & $0 / 24$ & $18 / 0$ \\
\hline & $\mathrm{R}=2,6$-diisopropylphenyl & $2 / 70$ & $5 / 0$ \\
\hline Without catalyst & - & $0 /$ trace & - \\
\hline
\end{tabular}

a 1 eq. of $y$-terpinene $\left(0.3 \cdot 10^{-3} \mathrm{~mol}\right)$ was added as a radical reaction inhibitor. 


\section{S3. Additional Data on Reaction Scope}

Scheme S1. (IMes) Pd(acac)Cl-Mediated hydrothiolation of alkynes.

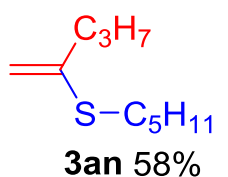

$50{ }^{\circ} \mathrm{C} 4 \mathrm{~h}$

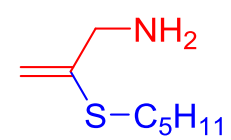

3ao $71 \%$

$100{ }^{\circ} \mathrm{C} 4 \mathrm{~h}$

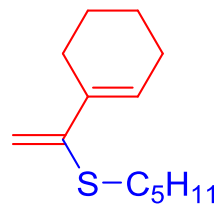

3ap $63 \%$

$100{ }^{\circ} \mathrm{C} 4 \mathrm{~h}$

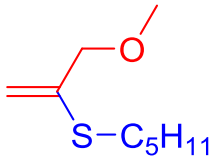

3 aq $63 \%$ $100{ }^{\circ} \mathrm{C} 4 \mathrm{~h}$

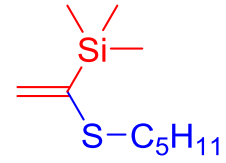

3ar $0 \%$

$100{ }^{\circ} \mathrm{C} 4 \mathrm{~h}$

The data on the reaction scope is provided on Scheme 2 of the manuscript and corresponding discussion is given in the text.

\section{S4. Mechanistic Studies}

Stepwise modeling of the hydrothiolation of alkynes was performed to investigate the reaction mechanism. We found out that the initial catalyst activation proceeds upon reaction with thiol. The equimolar mixing of (IMes) $\mathrm{Pd}(\mathrm{acac}) \mathrm{Cl}(\mathrm{I})$ with $\mathrm{Et}_{3} \mathrm{~N}$ and 2-methyl-2butyl-3-ol (2b) did not lead to any reactivity according to ${ }^{1} \mathrm{H}$ NMR and ESI-HRMS analyses. However, the reaction with thiol yielded a set of new signals in the proton spectrum. A series of test reactions between thiol (i.e., thiophenol (1b), 1-pentanethiol (1a), tert-butylthiol (1c)) and (IMes)Pd(acac)Cl (I) were carried out (Scheme S2). All of the reactions were monitored using NMR and ESI-HRMS.

Scheme S2. Model reaction of thiol and (IMes)Pd(acac)Cl.

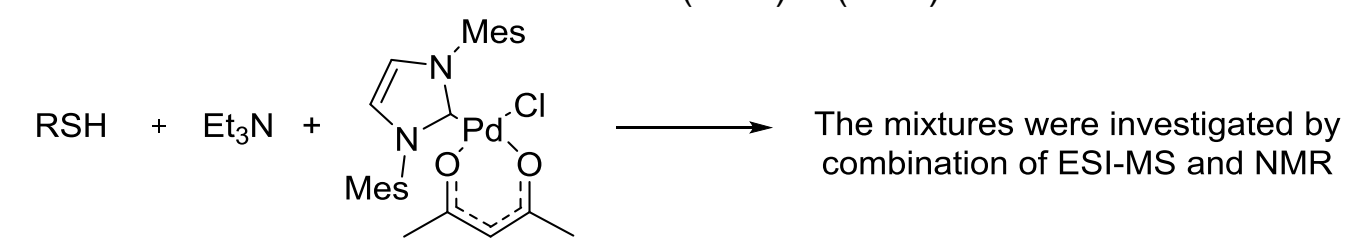

$\mathrm{R}=\mathrm{Ph}(1 \mathrm{~b}) ; n-\mathrm{C}_{5} \mathrm{H}_{11}(1 \mathrm{a}) ; t-\mathrm{Bu}(1 \mathrm{c})$ 


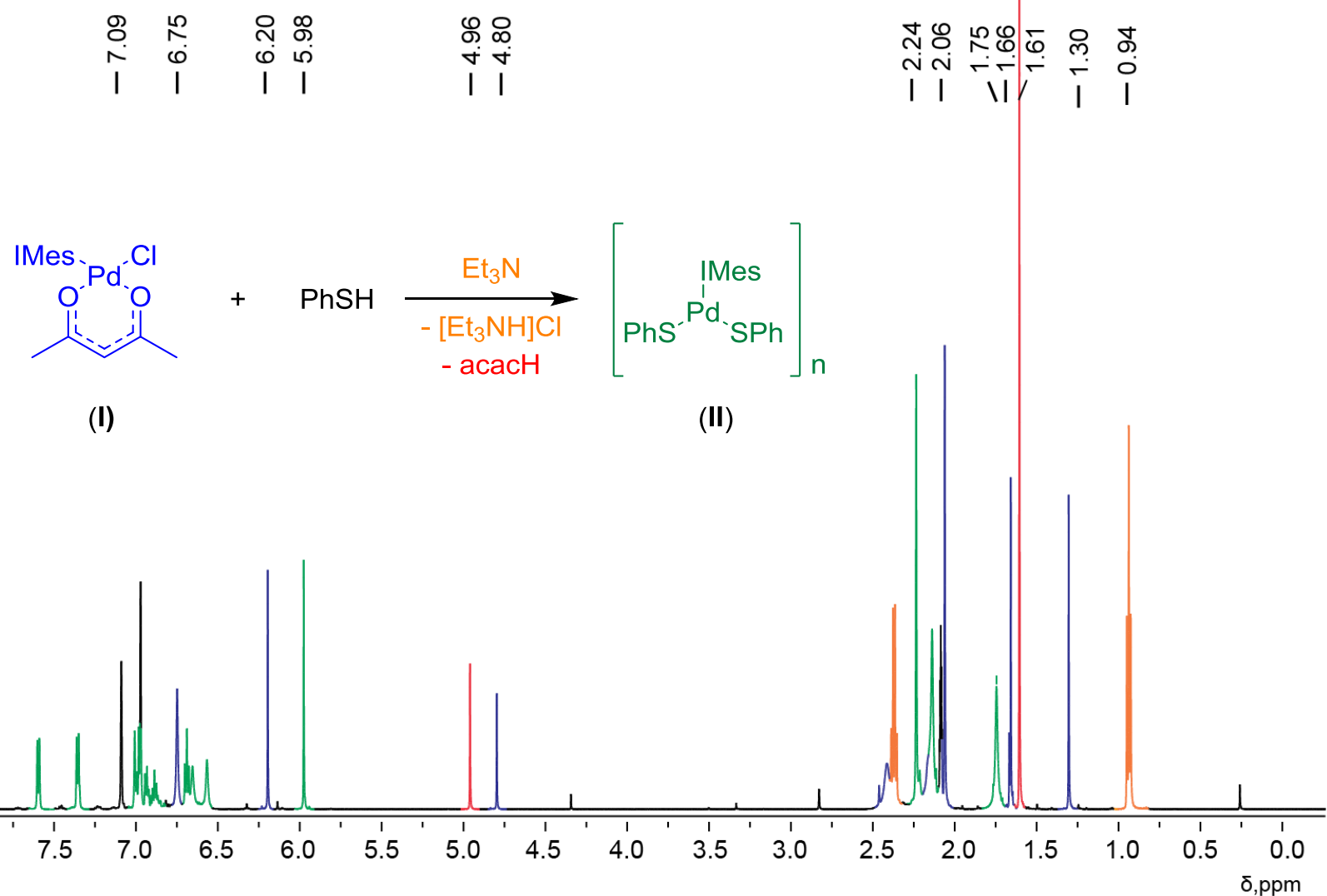

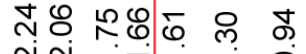

II Tili i i

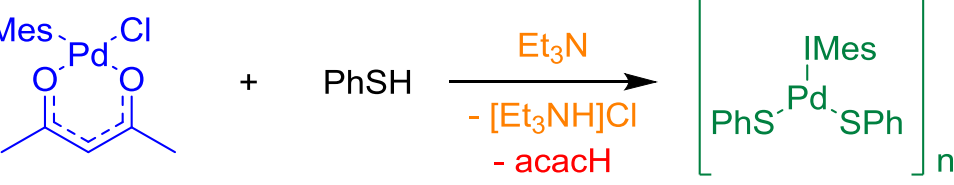

(l)

(II)

Figure S1. ${ }^{1} \mathrm{H}$ NMR spectrum (tol- $d_{8}, 500.1 \mathrm{MHz}$ ) of the equimolar reaction I + PhSH $\rightarrow$ II. Initial catalyst (IMes) $\mathrm{Pd}(\mathrm{acac}) \mathrm{Cl}$ (I) is shown in blue, dithiolate complex (II) is shown in green, $\mathrm{Et}_{3} \mathrm{~N}-$ orange, and red color indicates free actetylacetone.

In the reaction of 1 equiv of $\mathrm{PhSH}(\mathbf{1 b})$ with 1 equiv of (IMes) $\mathrm{Pd}(\mathrm{acac}) \mathrm{Cl}$ and 1 equiv of $\mathrm{Et}_{3} \mathrm{~N}$, half of the initial complex was converted to the dithiolate $\mathrm{Pd}-\mathrm{NHC}$ complex (Figure S1), which indicates substitution of both ligands (i.e., acac and $\mathrm{Cl}$ ) in the initial complex (I). The appearance of free acetylacetone was observed by ${ }^{1} \mathrm{H}$ NMR. After addition of the second equivalent of thiophenol (1) $\mathbf{b})$, the initial complex (I) was completely converted to (II), and this reaction progress was monitored with ${ }^{1} \mathrm{H}$ NMR. The final mixture was also analyzed with ESI-MS (Figure S2. a), where (IMes)Pd-containing species were observed, including $\quad \mathrm{Pd}(\mathrm{IMes})(\mathrm{SPh}), \quad \mathrm{Pd}_{2}(\mathrm{IMes})(\mathrm{SPh}), \quad \mathrm{Pd}_{2}(\mathrm{IMes})_{2}(\mathrm{SPh})_{2}$, $\mathrm{Pd}_{2}(\mathrm{IMes})_{2}(\mathrm{SPh})_{2} \mathrm{Cl}, \mathrm{Pd}_{2}(\mathrm{IMes})_{2}(\mathrm{SPh})_{3}$ and $\mathrm{Pd}_{2}(\mathrm{IMes})_{2}(\mathrm{SPh})_{4}$. 


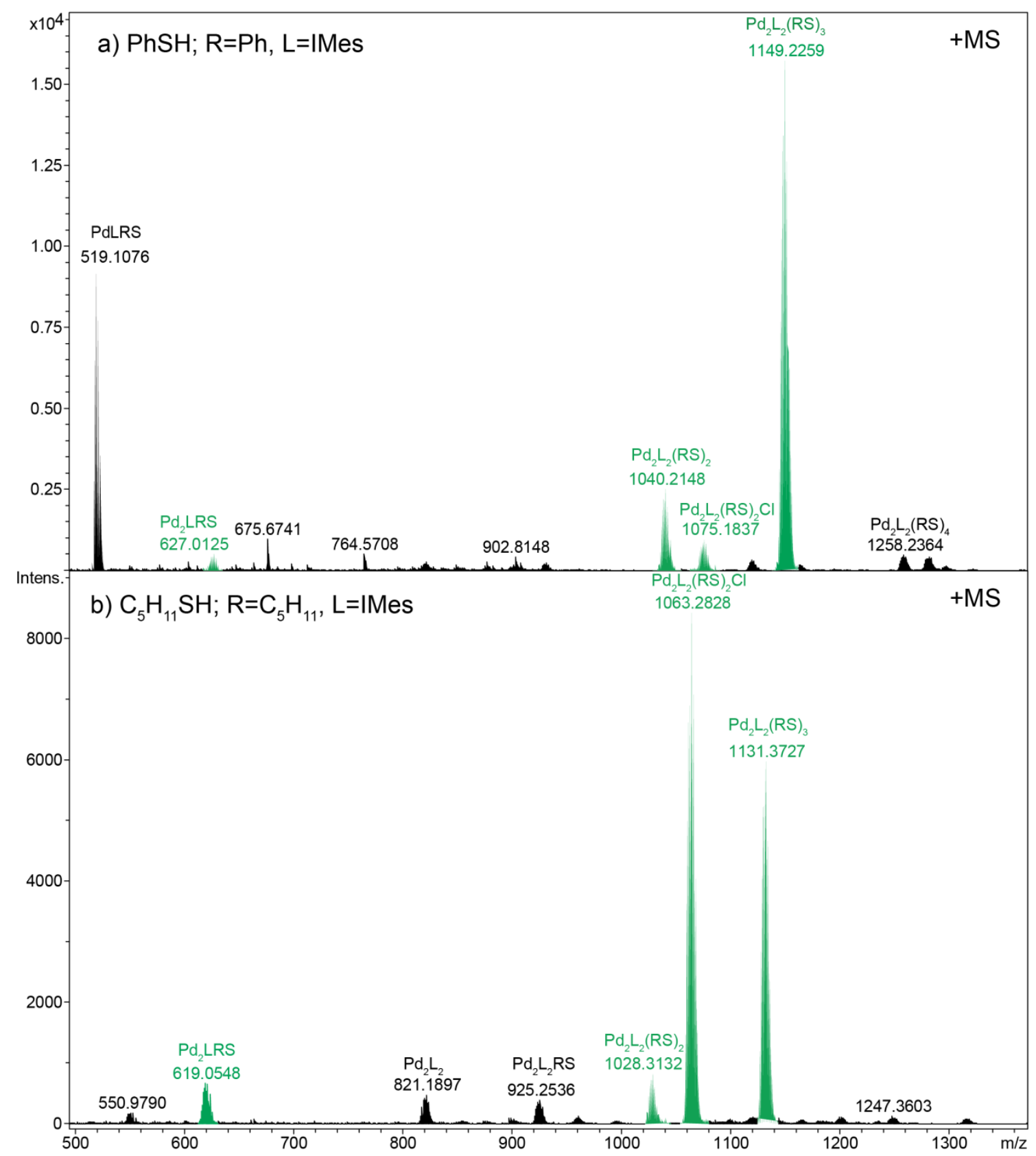

Figure S2. ESI-MS spectrum registered for the a) $\mathrm{PhSH}_{+} \mathrm{Et}_{3} \mathrm{~N}+(\mathrm{IMes}) \mathrm{Pd}(\mathrm{acac}) \mathrm{Cl}$ and b) $\mathrm{C}_{5} \mathrm{H}_{11} \mathrm{SH}+\mathrm{Et}_{3} \mathrm{~N}+(\mathrm{IMes}) \mathrm{Pd}(\mathrm{acac}) \mathrm{Cl}$ mixtures. The Pd-containing ions, which have similar patterns, are show in green. The intensity is given in arbitrary units (a.u.).

The same procedure was employed for aliphatic 1-pentanethiol (1a). The ${ }^{1} \mathrm{H}$ NMR spectrum for the reaction mixture appeared to be complicated but the molecular ions detected in the mass spectrum had similar patterns as compared to the thiophenol (1) case (Figure S2. a, b). A different result was observed during the interaction of tertbutylthiol (1C) and (I) where a new (5.06 ppm) signal appeared in the ${ }^{1} \mathrm{H}$ NMR spectrum. In the ESI-MS spectrum of the reaction mixture (Figure S3), various Pd-containing ions were detected, including similar ions to those observed in the reaction of $\mathrm{PhSH}$. In the 
case of tert-butylthiol (1c), most likely only the chloride ligand was substituted in the initial precatalyst (I) to form a $\left[(\mathrm{IMes}) \mathrm{Pd}(\mathrm{acac})\left(\mathrm{SC}_{4} \mathrm{H}_{9}\right)\right]_{\mathrm{n}}$ type of complex.

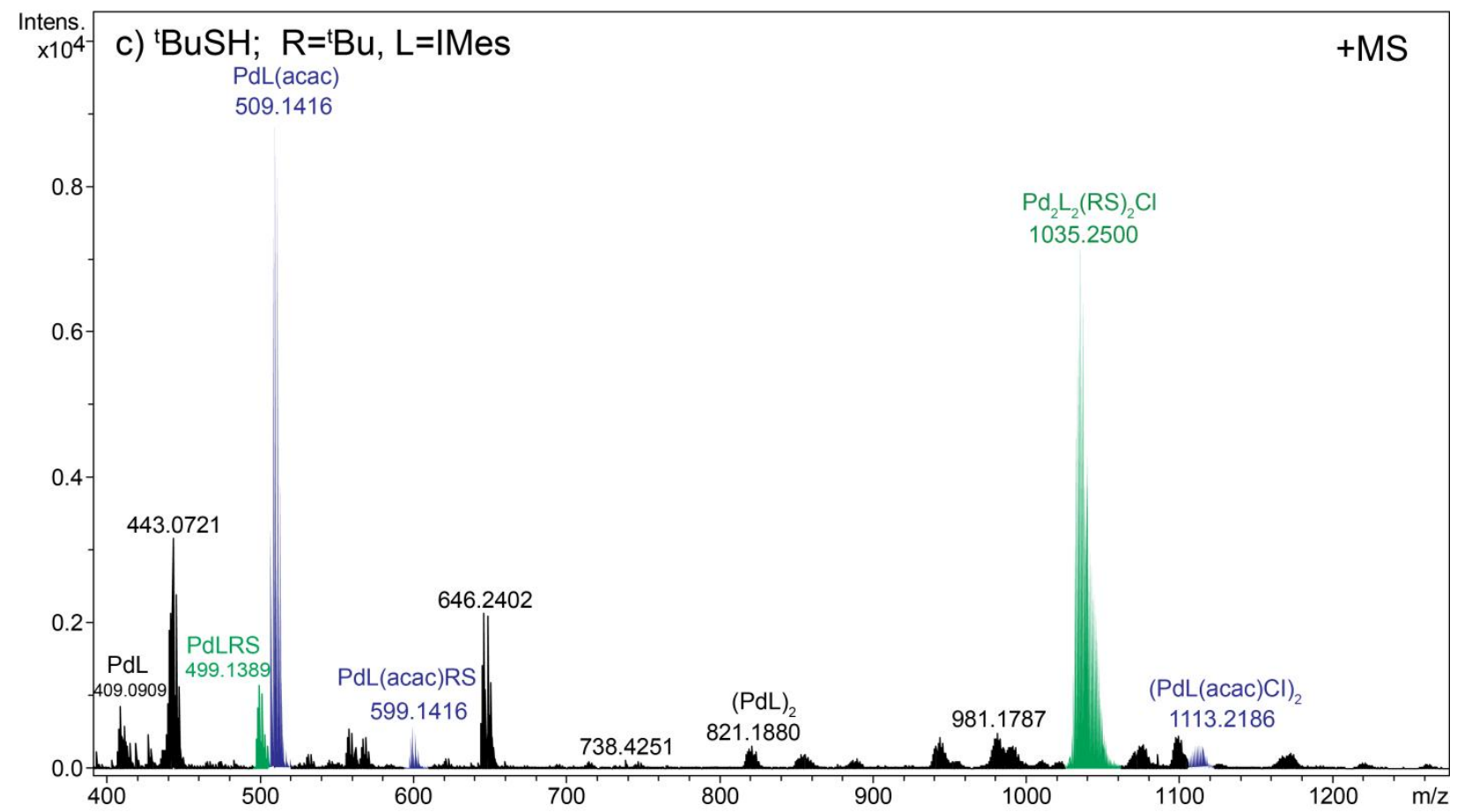

Figure S3. ESI-MS spectrum of the ${ }^{t} \mathrm{BuSH}_{+} \mathrm{Et}_{3} \mathrm{~N}+\mathrm{Pd}(\mathrm{I})$ reaction mixture. $\mathrm{Pd}$-containing ions, which contained acac, are shown in blue, and those ions that are similar to the $\mathrm{PhSH}$ case are shown in green. The intensity is given in arbitrary units (a.u.). 


\section{S5. Kinetic Measurements}

The method of initial rates was used for kinetic studies. The analysis was performed by integration of the ${ }^{1} \mathrm{H}$ NMR spectra with respect to 1,4-dioxane as internal standard. To deduce the hydrothiolation rate the reaction of 1-pentanthiol (1a) with 2methyl-3-butyn-2-ol (2b) mediated by (IMes) $\mathrm{Pd}(\mathrm{acac}) \mathrm{Cl}$ (I) was performed at room temperature in benzene- $d_{6}$, [1] and [2] were set to $0.2 \mathrm{M}$ unless otherwise indicated. The initial concentrations of thiol, alkyne and precatalyst were varied systematically and the data points were collected before the conversion level achieved $25 \%$. Data plot for this period of time demonstrates a linear trend (Figure S4), which indicates that reaction rate can be approximated as pseudo zero-order with respect to substrate concentrations. Reaction rate for each initial concentrations of reagents was determined according to eq. (1) as the slope $(m)$ from plot of [product] versus reaction time (in hours). $N_{t}$ was calculated according to eq. (2), where [catalyst] is initial concentration of precatalyst (I).

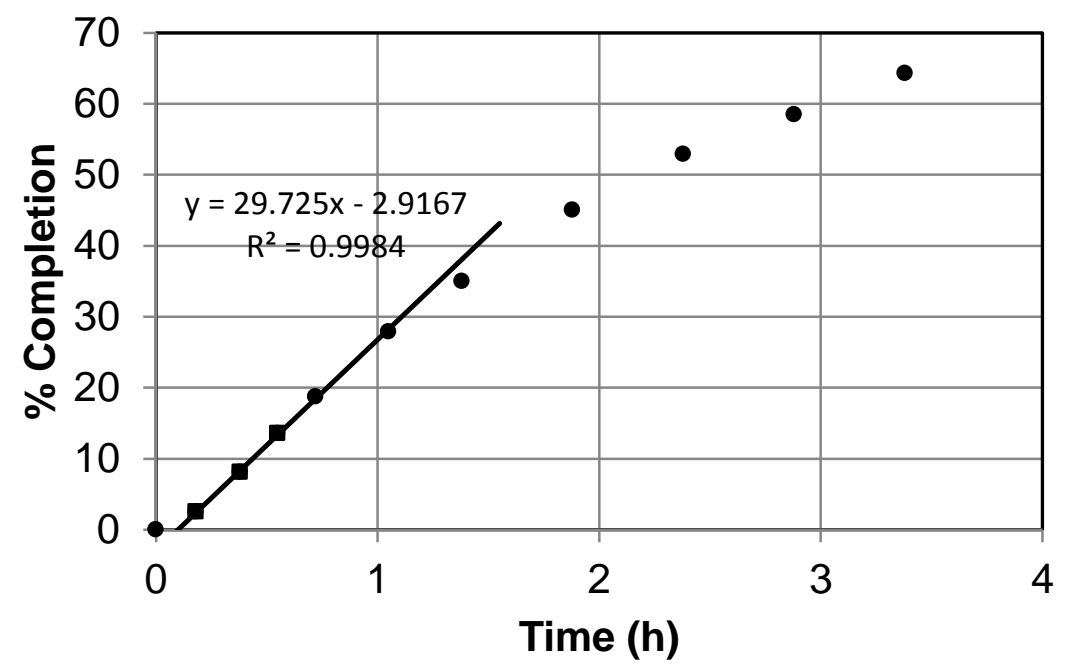

Figure S4. A plot of product formation against time for ( $\mathrm{IMes}) \mathrm{Pd}(\mathrm{acac}) \mathrm{Cl}$ mediated reaction between $\mathbf{2}$ and $\mathbf{1}$; the line shows the least square fit.

$$
\begin{gathered}
\text { [product }]=\mathrm{mt} \\
\mathrm{N}_{\mathrm{t}} \mathrm{h}^{-1}=\frac{\mathrm{m}}{[\text { catalyst }]_{0}}
\end{gathered}
$$

Reaction between $\mathbf{1 a}$ and $\mathbf{2 b}$ was studied to estimate the initial precatalyst concentration effect, where the [catalyst] $]_{0}$ concentration varied in the range $(0.4-16) \cdot 10^{-3} \mathrm{M}$ (Figure S5). Using larger precatalyst concentrations was limited with solubility of the palladium complex. To determine reaction order in [catalyst] the kinetic eq. (3) was 
plotted (Figure S5), where reaction order in catalyst was found as a diagram's slope (n). For the current reaction $\mathrm{n}=0.69$.

$$
\ln (\text { rate })=\mathrm{n} \cdot \ln \left([\text { catalyst }]_{0}\right)
$$

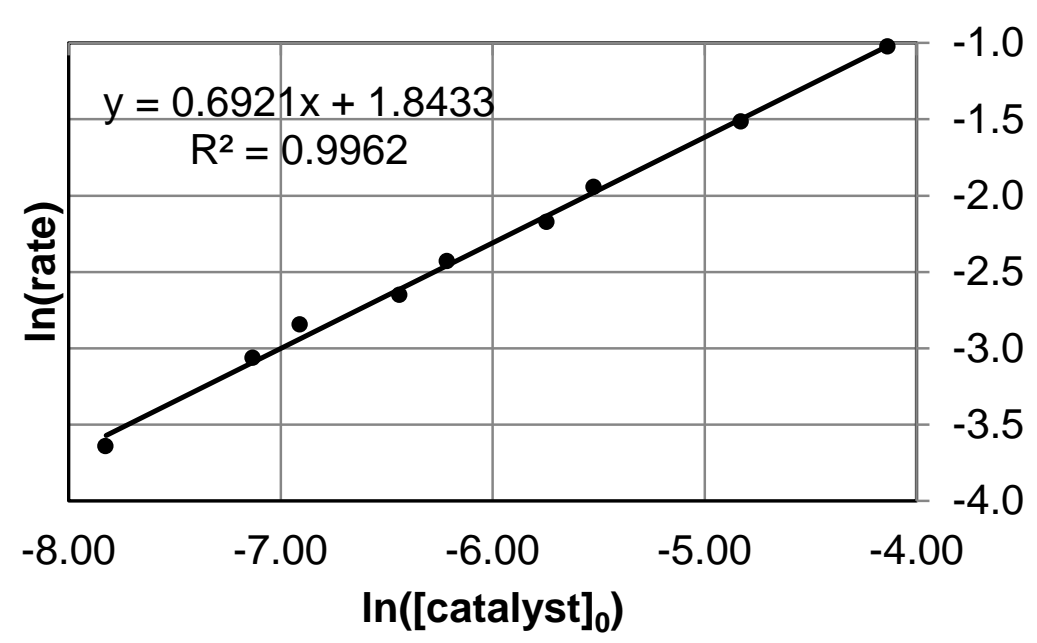

Figure S5. Graphic representation of reaction order in [catalyst] ; the line shows the least square fit.

The plot of [thiol] versus $\mathrm{N}_{t}$ over the concentration range of $0.05-1 \mathrm{M}$ demonstrated linear trend indicating first-order dependence of the reaction rate (Figure S6).

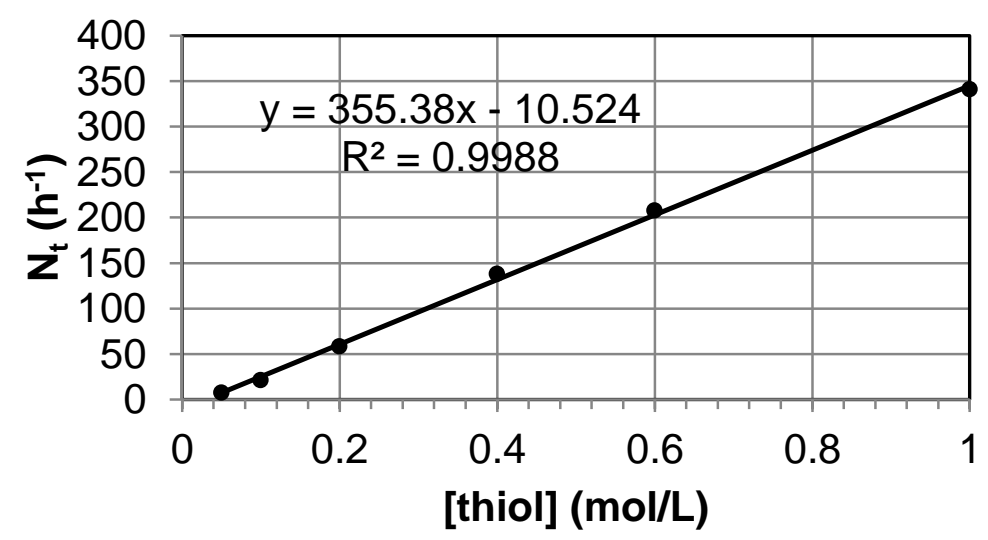

Figure S6. Plot of product formation rate versus [1a]; the line shows the least square fit. 


\section{S6. Deuterium Labeling Studies}

Hydrothiolation of 2-methyl-3-butyn-2-ol (2b) by PhSD (1 $\mathbf{b}-\boldsymbol{d})$ results in incorporation of deuterium in the $Z$-vinyl sulfide. The NMR yield of $Z-3 \mathbf{y}-\boldsymbol{d}$ is $38 \%$. The small amount of $E-3 \mathbf{y}-\boldsymbol{d}$ is connected with slow deuteriom exchange between the thiol and $\mathrm{CH}$ group of the alkyne. Fast deuterium/hydrogen scrambling between the thiol and $\mathrm{OH}$ group of the alkyne results in appearance of $\mathrm{PhSH}$ and furter formation of $3 \mathbf{y}(60 \%)$. Signal assignment in $\mathbf{3 y}$ was carried out using 2D NOESY NMR experiment.

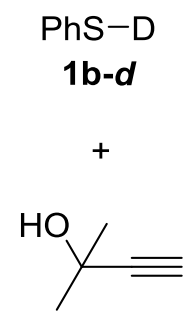

2b $\stackrel{\text { (IMes) } \mathrm{Pd}(\text { acac)Cl } 1 \mathrm{~mol} \%}{\longrightarrow}$

$\mathrm{Et}_{3} \mathrm{~N} 4 \mathrm{~mol} \%$ $\gamma$-terpinene 1 equiv $50{ }^{\circ} \mathrm{C} 1 \mathrm{~h} \mathrm{C}_{6} \mathrm{D}_{6}$
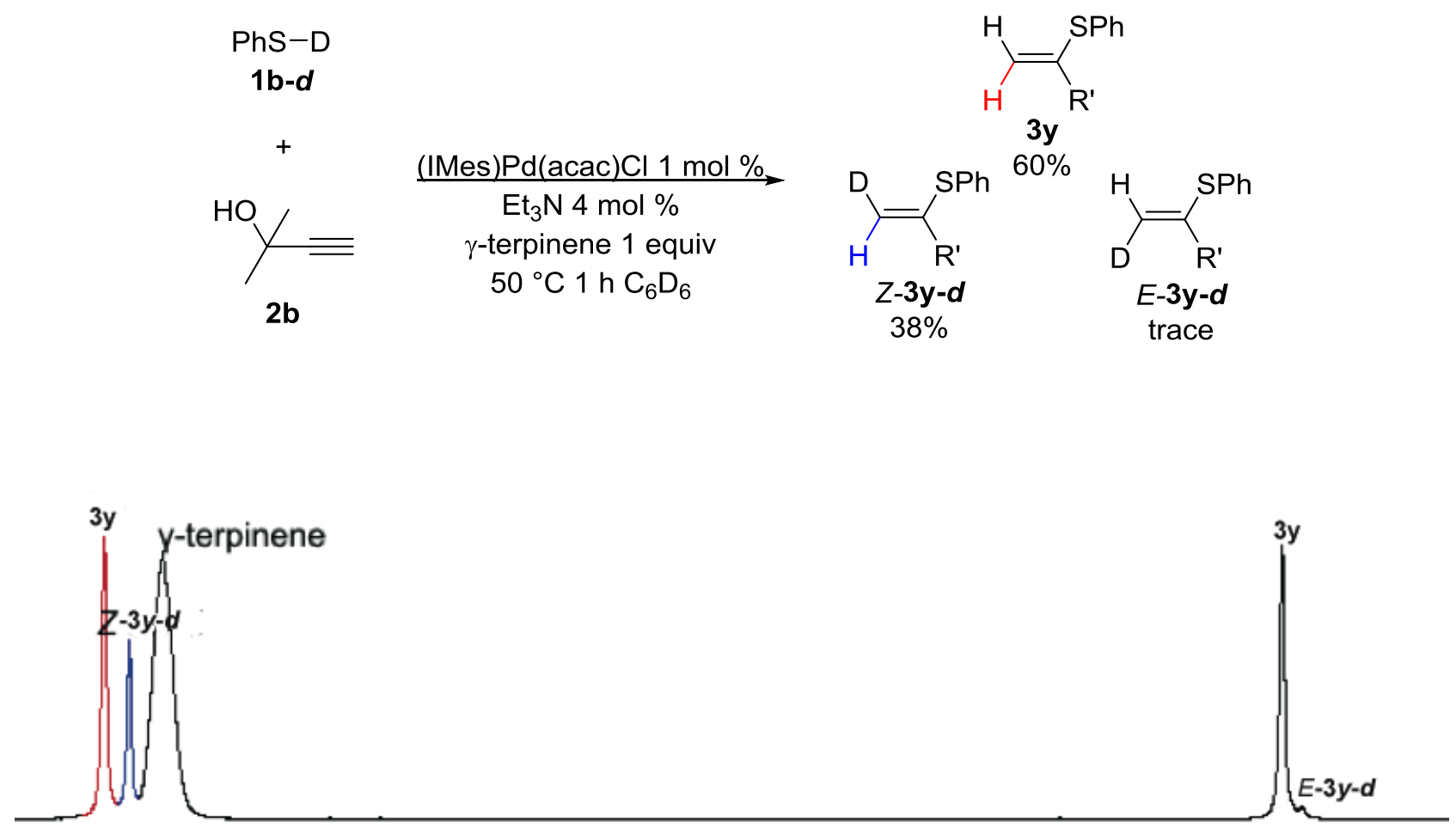

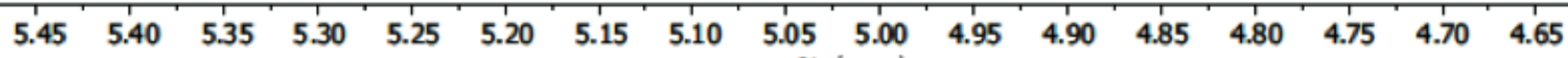

Figure S7. The ${ }^{1} \mathrm{H}$ NMR spectra of the reaction mixture in benzene- $d_{6}$ after $1 \mathrm{~h}$ at $50{ }^{\circ} \mathrm{C}$.

\section{S7. Theoretical Calculations of the Key Alkyne Insertion Stage}

To explain the experimentally observed reactivity differences between the binuclear and mononuclear palladium complexes theoretical calculations of the alkyne insertion into Pd-S bond was carried out at PBE1PBE/6-311G(d)\&SDD level of theory. The model system with the $R=M e, R^{\prime}=M e, L=1,3-$ dimethyl-1,3-dihydro-imidazole-2-ylidene was utilized in the calculations. 


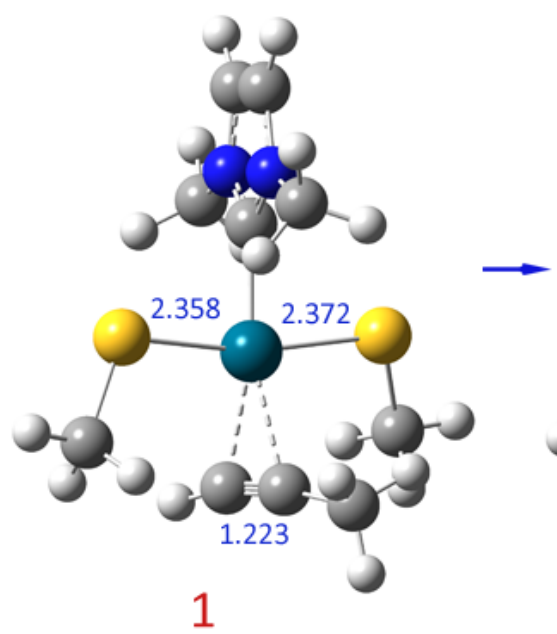

1

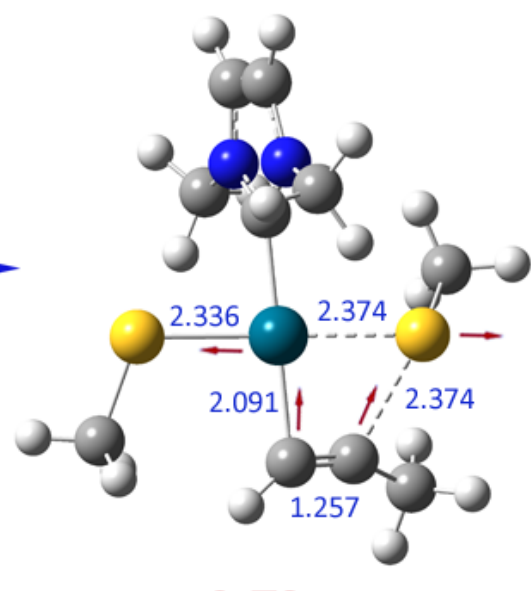

2-TS

(266i)

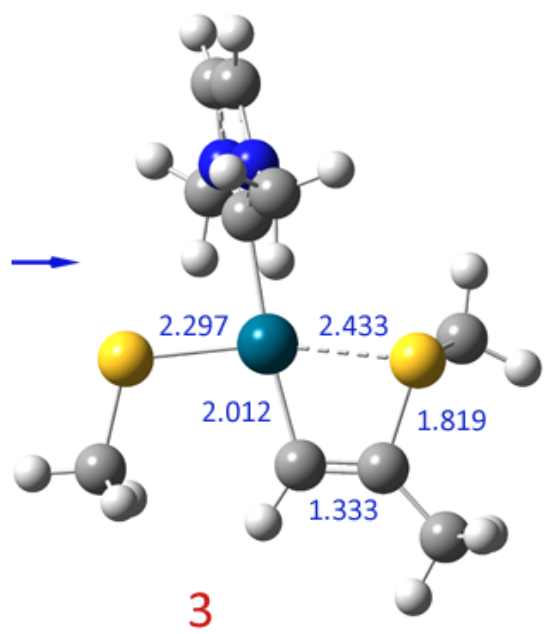

3

Figure S8. Optimized molecular structures of the intermediates, transition states and products of alkyne insertion reaction into Pd-S bond of the mononuclear complex at PBE1PBE/6$311 \mathrm{G}(\mathrm{d}) \&$ SDD level.

Palladium atom in the mononuclear complex has a vacant coordination site. In the first stage of the reaction the substrate molecule is coordinated to this vacancy and forms $\pi$-complex 1 , wherein the palladium atom is in square ligand environment. Favorable spatial location of the alkyne promotes insertion stage by rotation of the alkyne around L-Pd-C axis, which does not require a considerable amount of energy. The corresponding transition state 2-TS was located by geometry optimization procedure (Figure S8). Formation of $\mathrm{Pd}-\mathrm{C}$ and $\mathrm{C}-\mathrm{S}$ bonds in the transition state was accompanied by elongation of the Pd-S bond. In the product 3 the coordination of the sulfur atom with palladium was retained and additionally stabilized the reaction product. $\mathrm{A}$ small activation barrier of $\Delta \mathrm{G}^{\neq}=$ $12.9 \mathrm{kcal} / \mathrm{mol}$ and noticeable exothermic effect of $\Delta G=-10.7 \mathrm{kcal} / \mathrm{mol}$ were found in the calculations (Figure S9). 
<smiles>[R]S[S@]1([R])C([R])=C[Te]1(I)S[R]</smiles>

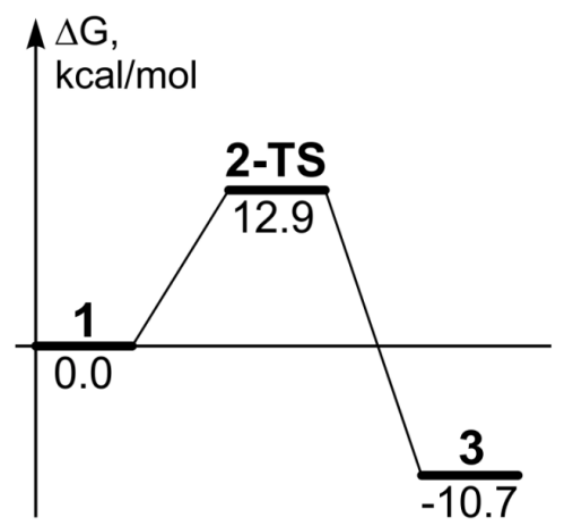

Figure S9. Potential energy surface of the alkyne insertion into the Pd-S bond of the mononuclear complex at PBE1PBE/6-311G(d)\&SDD level.
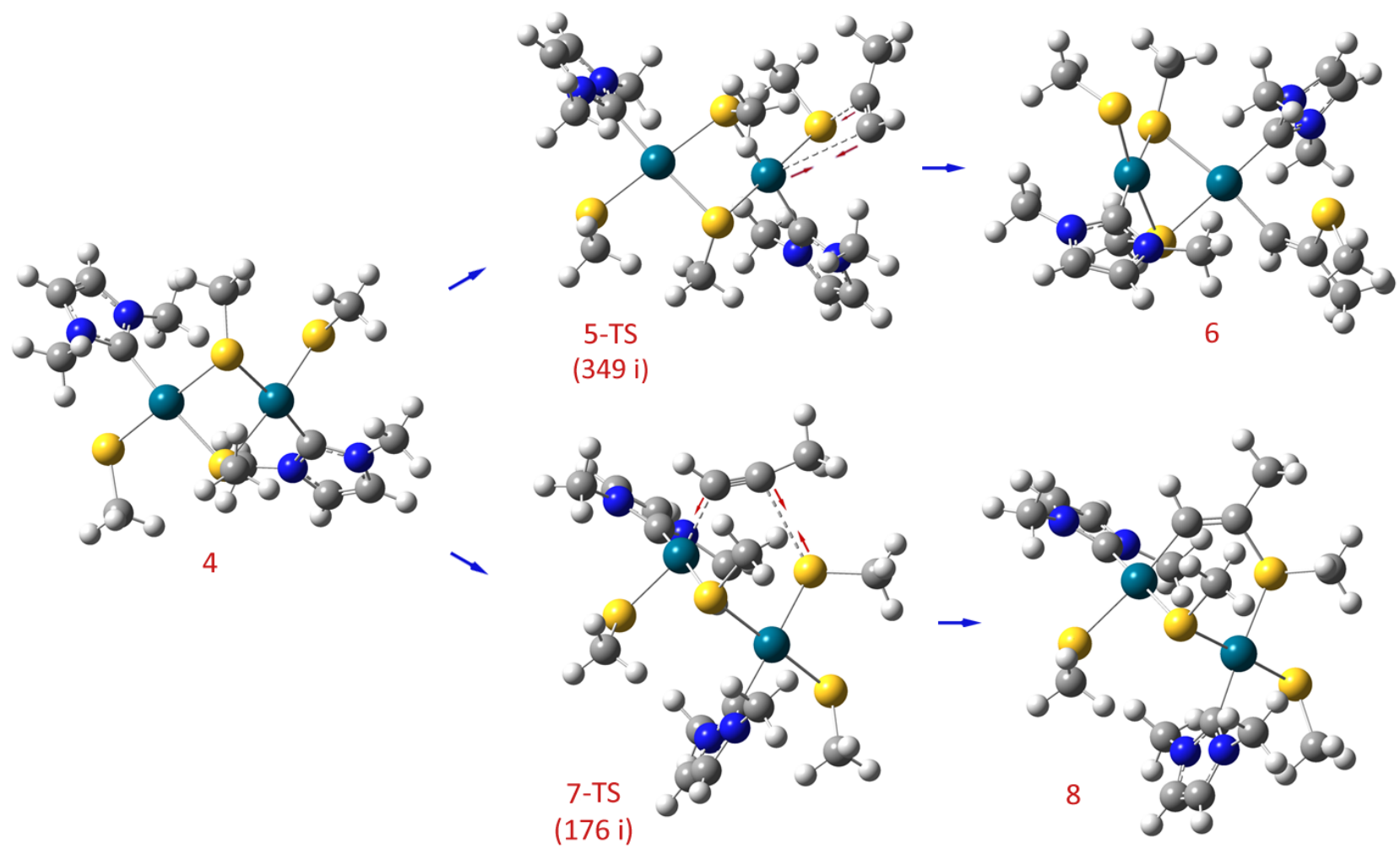

Figure S10. Optimized molecular structures of the intermediates, transition states and products of alkyne insertion into external $(5-T S, 6)$ and bridging $(7-T S, 8)$ Pd-S bonds of the binuclear complex, PBE1PBE/6-311G(d)\&SDD. 

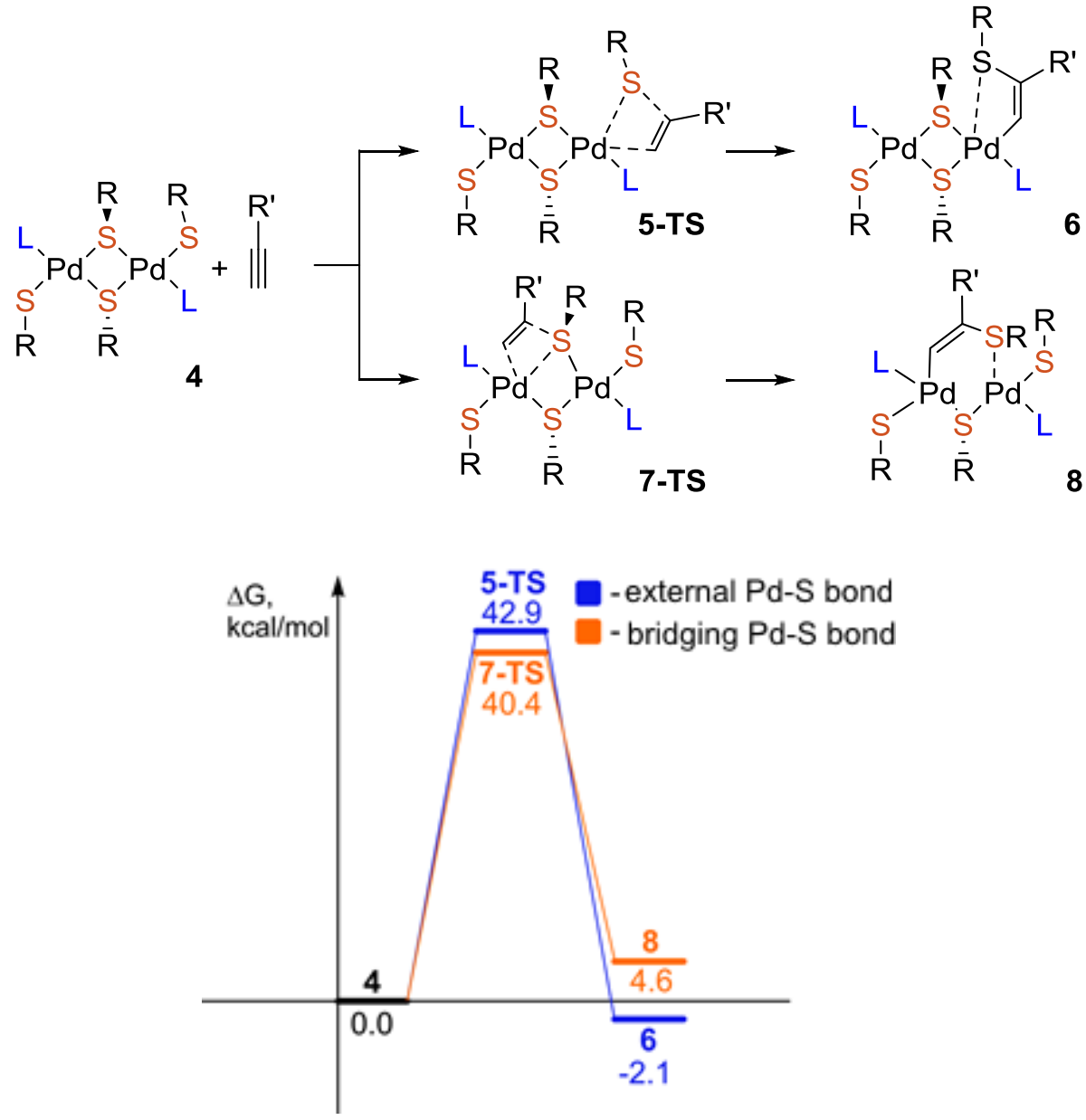

Figure S11. Potential energy surface of the alkyne insertion into external $(5-T S, 6)$ and bridging (7-TS, 8) Pd-S bonds of the binuclear complex at PBE1PBE/6-311G(d)\&SDD level.

In the binuclear complex there are two options for the alkyne insertion - into terminal or into bridging Pd-S bonds (Figure S10). Formation of a stable $\pi$-complex was not observed in contrast to complex 1 (Figure S8). The activation barrier of the insertion stages into the terminal $\mathrm{Pd}-\mathrm{S}$ bond $\left(\Delta \mathrm{G}^{\neq}=42.9 \mathrm{kcal} / \mathrm{mol}\right)$ and bridging $\mathrm{Pd}-\mathrm{S}$ bond $\left(\Delta \mathrm{G}^{\neq}=\right.$ $40.4 \mathrm{kcal} / \mathrm{mol}$ ) of binuclear complex were significantly higher as compared to the mononuclear complex (cf. Figure S9 and Figure S11). Combination of two factors (absence of coordination vacancy to bind the alkyne and higher intrinsic barrier of the insertion stage) apparently determines lower reactivity of the binuclear complex in the alkyne insertion reaction as compared to the mononuclear complex.

\section{Computational details}

The standard triple- $\zeta$ 6-311 $\mathrm{G}(\mathrm{d})$ basis set $^{1}$ for $\mathrm{H}, \mathrm{C}, \mathrm{N}, \mathrm{S}$ atoms and SDD basis set with Stuttgart/Dresden effective core potential ${ }^{2}$ for palladium atom were used for all 
calculations. The PBE1 $\mathrm{PBE}^{3}$ hybrid density functional was used for all optimizations and Hessian calculation procedures. All quantum-chemical calculations were performed using Gaussian 09 program. ${ }^{4}$ The model system with the $R=M e, R^{\prime}=M e, L=1,3-$ dimethyl-1,3dihydro-imidazole-2-ylidene was utilized in the calculations.

The normal coordinate analysis was performed for all optimized structures: reagents, intermediates, transition states and products and thermodynamic parameters were obtained for $298.15 \mathrm{~K}$ and $1 \mathrm{~atm}$.

The validation of the transition states structure was performed by IRC calculations ${ }^{5}$ and all the transition states (2-TS, 5-TS and 7-TS) correspond to the insertion of the alkyne molecule into the Pd-S bond. The initial geometry for binuclear complex 4 was obtained from the X-ray structure (Section S9).

\section{S8. Experimental Procedure}

\section{Catalytic Addition Reactions}

(IMes) $\mathrm{Pd}(\mathrm{acac}) \mathrm{Cl}\left(0.5 \times 10^{-5} \mathrm{~mol}, 0.0027 \mathrm{~g}\right), \mathrm{Et}_{3} \mathrm{~N}\left(2 \times 10^{-5} \mathrm{~mol}, 0.0020 \mathrm{~g}\right)$ and $\gamma^{-}$ terpinene $\left(0.5 \times 10^{-3} \mathrm{~mol}, 0.066 \mathrm{~g}\right)$ were added in a screw-capped test tube followed by the addition of alkyne $\left(0.5 \times 10^{-3} \mathrm{~mol}\right)$. Thiol $\left(0.5 \times 10^{-3} \mathrm{~mol}\right)$ was added to a stirred mixture and the vessel was flushed with argon to avoid thiol oxidation. The reaction temperature and time is given in the text (Scheme 2). After completion of the reaction, the reaction mixture was filtered through Celite 545 plug to remove the catalyst and washed with $\approx 5 \mathrm{~mL}$ of petroleum ether. Then the filtrate was evaporated under vacuum. In most of the studied cases the purity of the obtained products was $>90 \%$, additionally products can be purified with chromatography to obtain $\geq 98 \%$ purity. Dry column vacuum chromatography was made on silica gel 60-100 mesh with petroleum ether/EtOAc gradient elution. ${ }^{6}$ It is important to note that for several products (3a, 3c, 3d, 3f, 3j, 3k, 3I, 3an, 3ao, 3aq) even a trace amounts of acid initiated double bond isomerization.

NMR data for products $3 g_{,}{ }^{7} 3 k,{ }^{7} 3{ }^{8},{ }^{8} 3,{ }^{7} 3 q,{ }^{9} 3 y,{ }^{10} 3 z,{ }^{9} 3 a a^{11}$, 3af, ${ }^{12} 3 a o,{ }^{8} 3 a^{7}$ were in agreement with published literature data.

Hept-1-en-2-yl(pentyl)sulfane (3a). The product was obtained as light yellow oil after drying under vacuum.

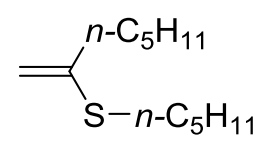

${ }^{1} \mathrm{H}$ NMR (500 MHz, $\left.\mathrm{C}_{6} \mathrm{D}_{6}\right) \delta$, ppm: $5.04(\mathrm{~s}, 1 \mathrm{H}), 4.73(\mathrm{~s}, 1 \mathrm{H}), 2.54$ (t, $J=7.4 \mathrm{~Hz}$, $2 \mathrm{H}), 2.25(\mathrm{t}, J=7.6 \mathrm{~Hz}, 2 \mathrm{H}), 1.64-1.57(\mathrm{~m}, 2 \mathrm{H}), 1.57-1.49(\mathrm{~m}, 2 \mathrm{H}), 1.28-1.11(\mathrm{~m}$, $8 \mathrm{H}), 0.85(\mathrm{t}, J=6.9 \mathrm{~Hz}, 3 \mathrm{H}), 0.80(\mathrm{t}, J=7.2 \mathrm{~Hz}, 3 \mathrm{H}) .{ }^{13} \mathrm{C}\left\{{ }^{1} \mathrm{H}\right\} \mathrm{NMR}\left(126 \mathrm{MHz}, \mathrm{C}_{6} \mathrm{D}_{6}\right) \delta$, 
ppm: 146.93, 104.95, 38.15, 31.57, 31.43, 29.06, 28.39, 22.86, 22.61, 14.24, 14.13. Anal. Calcd for $\mathrm{C}_{12} \mathrm{H}_{24} \mathrm{~S}, \%$ : C: 71.93, H: 12.07, S: 16.00; found, \%: C: 71.62, H: 12.05, S: 15.86.

2-methyl-3-(pentylthio)but-3-en-2-ol (3b). The product was obtained as light yellow oil after drying under vacuum.

${ }^{1} \mathrm{H}$ NMR $\left(500 \mathrm{MHz}, \mathrm{CDCl}_{3}\right) \delta$, ppm: $5.36(\mathrm{~s}, 1 \mathrm{H}), 4.75(\mathrm{~s}, 1 \mathrm{H}), 2.69$

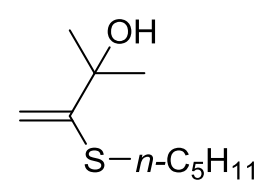
$(\mathrm{t}, \mathrm{J}=7.4 \mathrm{~Hz}, 2 \mathrm{H}), 2.00(\mathrm{~s}, 1 \mathrm{H}), 1.78-1.59(\mathrm{~m}, 2 \mathrm{H}), 1.44(\mathrm{~s}, 6 \mathrm{H}), 1.42-1.28(\mathrm{~m}, 4 \mathrm{H})$, 0.90 (t, J = 7.2 Hz, 3H). ${ }^{13} \mathrm{C}\left\{{ }^{1} \mathrm{H}\right\} \mathrm{NMR}\left(126 \mathrm{MHz}, \mathrm{CDCl}_{3}\right) \delta$, ppm: 154.67, 104.33, 73.89, $32.55,31.43,29.95,27.92,22.40,14.05$. HRMS (ESI) $\mathrm{m} / \mathrm{z}$ : $[\mathrm{M}+\mathrm{Na}]^{+}$calcd for $\mathrm{C}_{10} \mathrm{H}_{20} \mathrm{OS}$ 211.1127, found $211.1123(\Delta=1.9 \mathrm{ppm})$.

$\mathrm{N}, \mathrm{N}$-dimethyl-2-(pentylthio)prop-2-en-1-amine (3c). The product was obtained as light yellow oil after drying under vacuum.

${ }^{1} \mathrm{H}$ NMR $\left(500 \mathrm{MHz}, \mathrm{C}_{6} \mathrm{D}_{6}\right) \delta$, ppm: $5.25(\mathrm{~s}, 1 \mathrm{H}), 4.79(\mathrm{~s}, 1 \mathrm{H}), 3.01$

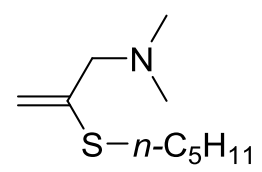
(s, 2H), $2.56(\mathrm{t}, \mathrm{J}=7.4 \mathrm{~Hz}, 2 \mathrm{H}), 2.13(\mathrm{~s}, 6 \mathrm{H}), 1.57-1.49(\mathrm{~m}, 2 \mathrm{H}), 1.26-1.11(\mathrm{~m}, 4 \mathrm{H})$, $0.79(\mathrm{t}, \mathrm{J}=7.2 \mathrm{~Hz}, 3 \mathrm{H}) .{ }^{13} \mathrm{C}\left\{{ }^{1} \mathrm{H}\right\}$ NMR (126 MHz, $\left.\mathrm{C}_{6} \mathrm{D}_{6}\right) \delta$, ppm: 145.66, 106.42, 66.33, 45.07, 31.62, 31.02, 28.44, 22.61, 14.13. HRMS (ESI) $\mathrm{m} / \mathrm{z}:[\mathrm{M}+\mathrm{H}]^{+}$calcd for $\mathrm{C}_{10} \mathrm{H}_{21} \mathrm{NS}$ 188.1467, found 188.1463 ( $\Delta=2.1 \mathrm{ppm})$. Anal. Calcd for $\mathrm{C}_{10} \mathrm{H}_{21} \mathrm{NS}, \%$ : C: $64.11, \mathrm{H}$ : 11.30, S: 17.11; found, \%: C: 64.06, H: 11.42, S: 17.04 .

5-(pentylthio)hex-5-enenitrile (3d). The product was obtained as brown oil after drying under vacuum.

${ }^{1} \mathrm{H}$ NMR $\left(500 \mathrm{MHz}, \mathrm{C}_{6} \mathrm{D}_{6}\right) \delta$, ppm: 4.87 (s, 1H), $4.62(\mathrm{~s}, 1 \mathrm{H}), 2.41$

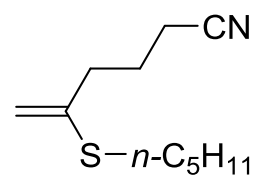
(t, J = 7.3 Hz, 2H), $2.02(\mathrm{t}, \mathrm{J}=7.2 \mathrm{~Hz}, 2 \mathrm{H}), 1.53-1.41(\mathrm{~m}, 4 \mathrm{H}), 1.39-1.32(\mathrm{~m}, 2 \mathrm{H}), 1.22$ - $1.10(\mathrm{~m}, 4 \mathrm{H}), 0.80(\mathrm{t}, \mathrm{J}=6.9 \mathrm{~Hz}, 3 \mathrm{H}) .{ }^{13} \mathrm{C}\left\{{ }^{1} \mathrm{H}\right\} \mathrm{NMR}\left(126 \mathrm{MHz}, \mathrm{C}_{6} \mathrm{D}_{6}\right) \delta, p p m: 144.14$, 119.02, 106.89, 36.29, 31.45, 31.32, 28.23, 24.54, 22.55, 15.63, 14.09. HRMS (ESI) $\mathrm{m} / \mathrm{z}$ : $[\mathrm{M}+\mathrm{H}]^{+}$calcd for $\mathrm{C}_{11} \mathrm{H}_{19} \mathrm{NS}$ 198.1311, found $198.1306(\Delta=2.5 \mathrm{ppm})$.

2-methyl-3-(pentylthio)but-3-en-2-yl acetate (3e). The product was obtained as yellow oil after drying under vacuum.

${ }^{1} \mathrm{H}$ NMR $\left(500 \mathrm{MHz}, \mathrm{C}_{6} \mathrm{D}_{6}\right) \delta$, ppm: $5.25(\mathrm{~s}, 1 \mathrm{H}), 4.76(\mathrm{~s}, 1 \mathrm{H}), 2.68(\mathrm{t}$,

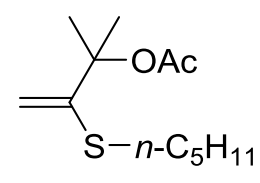
$J=7.4 \mathrm{~Hz}, 2 \mathrm{H}), 2.01(\mathrm{~s}, 3 \mathrm{H}), 1.67-1.60(\mathrm{~m}, 8 \mathrm{H}), 1.42-1.28(\mathrm{~m}, 4 \mathrm{H}), 0.89(\mathrm{t}, J=7.1 \mathrm{~Hz}$, $3 \mathrm{H}) .{ }^{13} \mathrm{C}\left\{{ }^{1} \mathrm{H}\right\} \mathrm{NMR}\left(126 \mathrm{MHz}, \mathrm{CDCl}_{3}\right) \delta$, ppm: 169.70, 151.32, 104.31, 82.35, 31.79, 31.45, 27.69, 27.66, 22.38, 22.14, 14.04. HRMS (ESI) $\mathrm{m} / \mathrm{z}$ : $[\mathrm{M}+\mathrm{H}]^{+}$calcd for $\mathrm{C}_{12} \mathrm{H}_{22} \mathrm{O}_{2} \mathrm{~S}$ 231.1413 , found 231.1410 ( $\Delta=1.3 \mathrm{ppm})$.

(6-chlorohex-1-en-2-yl)(pentyl)sulfane (3f). The product was obtained as yellow oil after drying under vacuum.

${ }^{1} \mathrm{H}$ NMR $\left(500 \mathrm{MHz}, \mathrm{C}_{6} \mathrm{D}_{6}\right) \delta$, ppm $4.94(\mathrm{~s}, 1 \mathrm{H}), 4.68(\mathrm{~s}, 1 \mathrm{H}), 3.07$ (t, J

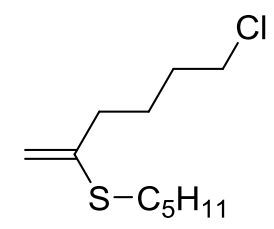


$=6.6 \mathrm{~Hz}, 2 \mathrm{H}), 2.49(\mathrm{t}, \mathrm{J}=7.4 \mathrm{~Hz}, 2 \mathrm{H}), 2.08(\mathrm{t}, \mathrm{J}=7.3 \mathrm{~Hz}, 2 \mathrm{H}), 1.57-1.41(\mathrm{~m}, 6 \mathrm{H}), 1.24-$ $1.10(\mathrm{~m}, 4 \mathrm{H}), 0.80(\mathrm{t}, \mathrm{J}=7.1 \mathrm{~Hz}, 3 \mathrm{H}) .{ }^{13} \mathrm{C}\left\{{ }^{1} \mathrm{H}\right\} \mathrm{NMR}\left(126 \mathrm{MHz}, \mathrm{C}_{6} \mathrm{D}_{6}\right) \delta, \mathrm{ppm}: 146.05$, 105.34, 44.63, 37.14, 32.03, 31.52, 31.36, 28.28, 26.33, 22.59, 14.13.

(1-cyclohexylvinyl)(pentyl)sulfane (3g). The product was obtained as yellow oil after drying under vacuum. Spectral data are in agreement with previously reported. ${ }^{7}$

${ }^{1} \mathrm{H}$ NMR $\left(500 \mathrm{MHz}, \mathrm{C}_{6} \mathrm{D}_{6}\right) \delta$, ppm: $5.07(\mathrm{~s}, 1 \mathrm{H}), 4.67(\mathrm{~s}, 1 \mathrm{H}), 2.53$

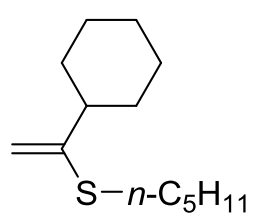
(t, $J=7.0 \mathrm{~Hz}, 2 \mathrm{H}), 2.14(\mathrm{t}, J=11.5 \mathrm{~Hz}, 1 \mathrm{H}), 1.98(\mathrm{~d}, J=12.5 \mathrm{~Hz}, 2 \mathrm{H}), 1.68(\mathrm{~d}, J=12.5$ $\mathrm{Hz}, 2 \mathrm{H}), 1.54$ (t, $J=7.5 \mathrm{~Hz}, 3 \mathrm{H}), 1.40(\mathrm{~m}, 2 \mathrm{H}), 1.25-1.02(\mathrm{~m}, 7 \mathrm{H}), 0.80(\mathrm{t}, J=7.0 \mathrm{~Hz}, 3 \mathrm{H})$.

1-(1-(hexylthio)vinyl)cyclohexan-1-ol (3h). The product was obtained as light yellow oil after dry column vacuum chromatography.

${ }^{1} \mathrm{H}$ NMR $\left(500 \mathrm{MHz}, \mathrm{CDCl}_{3}\right) \delta$, ppm: $5.39(\mathrm{~s}, 1 \mathrm{H}), 4.79(\mathrm{~s}, 1 \mathrm{H}), 2.68(\mathrm{t}$,

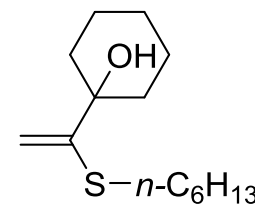
$\mathrm{J}=7.4 \mathrm{~Hz}, 2 \mathrm{H}), 1.80-1.59(\mathrm{~m}, 10 \mathrm{H}), 1.59-1.51(\mathrm{~m}, 2 \mathrm{H}), 1.46-1.36(\mathrm{~m}, 2 \mathrm{H}), 1.35-$ $1.16(\mathrm{~m}, 5 \mathrm{H}), 0.88(\mathrm{t}, \mathrm{J}=6.8 \mathrm{~Hz}, 3 \mathrm{H}) .{ }^{13} \mathrm{C}\left\{{ }^{1} \mathrm{H}\right\} \mathrm{NMR}\left(126 \mathrm{MHz}, \mathrm{CDCl}_{3}\right) \delta$, ppm: 154.92, 104.89, 74.59, 37.23, 32.59, 31.54, 28.96, 28.23, 25.62, 22.64, 22.14, 14.12. HRMS (ESI) $\mathrm{m} / \mathrm{z}:[\mathrm{M}+\mathrm{H}]^{+}$calcd for $\mathrm{C}_{14} \mathrm{H}_{26} \mathrm{OS} 243.1777$, found $243.1773(\Delta=1.6 \mathrm{ppm})$. Anal. Calcd for $\mathrm{C}_{14} \mathrm{H}_{26} \mathrm{OS}, \%$ : C: 69.36, H: 10.81, S: 13.22; found, \%: C: 69.40, H: 10.78, S: 13.31.

2-(pentylthio)-1,1-diphenylprop-2-en-1-ol (3i). The product was obtained as light brown oil after dry column vacuum chromatography.

${ }^{1} \mathrm{H}$ NMR $\left(500 \mathrm{MHz}, \mathrm{CDCl}_{3}\right) \delta$, ppm: 7.40 (d, $\left.J=7.4 \mathrm{~Hz}, 4 \mathrm{H}\right), 7.33$

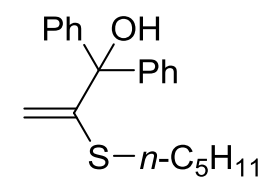
$-7.25(\mathrm{~m}, 6 \mathrm{H}), 5.27(\mathrm{~s}, 1 \mathrm{H}), 4.99(\mathrm{~s}, 1 \mathrm{H}), 2.73(\mathrm{t}, J=7.4 \mathrm{~Hz}, 2 \mathrm{H}), 1.67-1.59(\mathrm{~m}, 2 \mathrm{H})$, $1.37-1.27(\mathrm{~m}, 4 \mathrm{H}), 0.88(\mathrm{t}, J=7.0 \mathrm{~Hz}, 3 \mathrm{H}) .{ }^{13} \mathrm{C}\left\{{ }^{1} \mathrm{H}\right\} \mathrm{NMR}\left(126 \mathrm{MHz}, \mathrm{CDCl}_{3}\right) \delta$, ppm: $152.59,144.30,127.93,127.61,115.21,82.80,34.30,31.26,28.33,22.36,14.04$. HRMS (ESI) $\mathrm{m} / \mathrm{z}:[\mathrm{M}+\mathrm{Na}]^{+}$calcd for $\mathrm{C}_{20} \mathrm{H}_{24} \mathrm{OS} 335.1440$, found 335.1444 ( $\left.\Delta=1.2 \mathrm{ppm}\right)$.

4-(hexylthio)pent-4-en-2-ol (3j). The product was obtained as yellow oil after drying under vacuum.

${ }^{1} \mathrm{H}$ NMR $\left(500 \mathrm{MHz}, \mathrm{C}_{6} \mathrm{D}_{6}\right) \delta$, ppm: $4.98(\mathrm{~s}, 1 \mathrm{H}), 4.69(\mathrm{~s}, 1 \mathrm{H}), 4.01-$

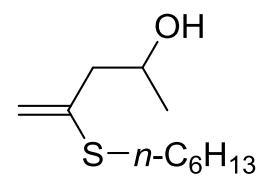
$3.97(\mathrm{~m}, 1 \mathrm{H}), 2.46(\mathrm{t}, J=7.3 \mathrm{~Hz}, 2 \mathrm{H}), 2.33-2.21(\mathrm{~m}, 2 \mathrm{H}), 1.60(\mathrm{~s}, 1 \mathrm{H}), 1.51-1.43(\mathrm{~m}$, $2 \mathrm{H}), 1.25-1.15(\mathrm{~m}, 4 \mathrm{H}), 1.14-1.07(\mathrm{~m}, 5 \mathrm{H}), 0.84(\mathrm{t}, J=7.2 \mathrm{~Hz}, 3 \mathrm{H}) .{ }^{13} \mathrm{C}\left\{{ }^{1} \mathrm{H}\right\}$ NMR $(126$ $\left.\mathrm{MHz}, \mathrm{C}_{6} \mathrm{D}_{6}\right) \delta$, ppm: 143.53, 107.37, 66.53, 48.14, 31.67, 31.54, 29.03, 28.43, 22.87, 22.85, 14.20. HRMS (ESI) $\mathrm{m} / \mathrm{z}:[\mathrm{M}+\mathrm{H}]^{+}$calcd for $\mathrm{C}_{11} \mathrm{H}_{22} \mathrm{OS} 203.1464$, found $203.1458(\Delta=$ $3.0 \mathrm{ppm})$.

Hex-1-en-2-yl(pentyl)sulfane (3k). The product was obtained as yellow oil after drying under vacuum. Spectral data are in agreement

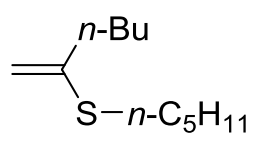


with previously reported. ${ }^{7}$

${ }^{1} \mathrm{H}$ NMR (500 MHz, $\left.\mathrm{C}_{6} \mathrm{D}_{6}\right) \delta$, ppm: $5.03(\mathrm{~s}, 1 \mathrm{H}), 4.73(\mathrm{~s}, 1 \mathrm{H}), 2.53$ (t, $J=7.2 \mathrm{~Hz}$, 2H), 2.25 (t, $J=7.6 \mathrm{~Hz}, 2 \mathrm{H}), 1.63-1.47(\mathrm{~m}, 4 \mathrm{H}), 1.34-1.08(\mathrm{~m}, 6 \mathrm{H}), 0.85$ (t, $J=7.4 \mathrm{~Hz}$, $3 \mathrm{H}), 0.80$ (t, $J=7.1 \mathrm{~Hz}, 3 \mathrm{H})$.

Pentyl(3-phenylprop-1-en-2-yl)sulfane (3I). The product was obtained as yellow oil after drying under vacuum. Spectral data are in agreement with previously reported. ${ }^{8}$

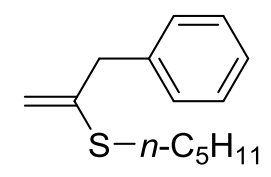

${ }^{1} \mathrm{H}$ NMR (500 MHz, $\left.\mathrm{C}_{6} \mathrm{D}_{6}\right) \delta$, ppm: 7.20-7.16 (m, 2H), 7.15-7.10 (m, $\left.2 \mathrm{H}\right)$, 7.06-7.01 (m, 1H), $4.96(\mathrm{~s}, 1 \mathrm{H}), 4.73(\mathrm{~s}, 1 \mathrm{H}), 3.44(\mathrm{~s}, 2 \mathrm{H}), 2.42(\mathrm{t}, J=7.5 \mathrm{~Hz}, 2 \mathrm{H}), 1.44-1.36(\mathrm{~m}$, $2 \mathrm{H}), 1.13-1.01(\mathrm{~m}, 4 \mathrm{H}), 0.75(\mathrm{t}, J=7.0 \mathrm{~Hz}, 3 \mathrm{H})$.

Pentyl(1-phenylvinyl)sulfane $(3 \mathrm{~m})$. The product was obtained as light brown oil after dry column vacuum chromatography. Spectral data are in agreement with previously reported. ${ }^{7}$

${ }^{1} \mathrm{H}$ NMR $\left(500 \mathrm{MHz}, \mathrm{CDCl}_{3}\right) \delta$, ppm: $7.56(\mathrm{~d}, J=6.6 \mathrm{~Hz}, 2 \mathrm{H}), 7.38-$

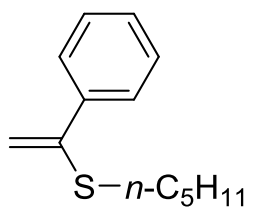
$7.29(\mathrm{~m}, 3 \mathrm{H}), 5.46(\mathrm{~s}, 1 \mathrm{H}), 5.17(\mathrm{~s}, 1 \mathrm{H}), 2.70(\mathrm{t}, J=7.4 \mathrm{~Hz}, 2 \mathrm{H}), 1.69-1.61(\mathrm{~m}, 2 \mathrm{H}), 1.44$ - $1.30(\mathrm{~m}, 4 \mathrm{H}), 0.91$ (t, $J=7.2 \mathrm{~Hz}, 3 \mathrm{H}) .{ }^{1} \mathrm{H}$ NMR $\left(500 \mathrm{MHz}, \mathrm{C}_{6} \mathrm{D}_{6}\right) \delta$, ppm: 7.63 (d, $J=7.5$ $\mathrm{Hz}, 2 \mathrm{H}$ ), 7.17-7.02 (m, 3H), 5.41 (s, 1H), $5.14(\mathrm{~s}, 1 \mathrm{H}), 2.48$ (t, $J=7.5 \mathrm{~Hz}, 2 \mathrm{H}), 1.48$ (t, $J=$ $7.5 \mathrm{~Hz}, 2 \mathrm{H}), 1.20-1.05(\mathrm{~m}, 4 \mathrm{H}), 0.77(\mathrm{t}, J=7.0 \mathrm{~Hz}, 3 \mathrm{H})$.

2-(hexylthio)-3-methylpent-1-en-3-ol (3n). The product was obtained as yellow oil after dry column vacuum chromatography.

${ }^{1} \mathrm{H}$ NMR $\left(500 \mathrm{MHz}, \mathrm{CDCl}_{3}\right) \delta$, ppm: $5.33(\mathrm{~s}, 1 \mathrm{H}), 4.80(\mathrm{~s}, 1 \mathrm{H}), 2.69(\mathrm{t}$,

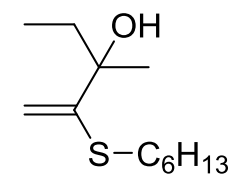
$\mathrm{J}=7.4 \mathrm{~Hz}, 2 \mathrm{H}), 1.87$ (br. s, $1 \mathrm{H}), 1.79-1.60(\mathrm{~m}, 4 \mathrm{H}), 1.46-1.36(\mathrm{~m}, 5 \mathrm{H}), 1.35-1.25(\mathrm{~m}$, $4 \mathrm{H}), 0.89(\mathrm{t}, \mathrm{J}=6.9 \mathrm{~Hz}, 3 \mathrm{H}), 0.85(\mathrm{t}, \mathrm{J}=7.4 \mathrm{~Hz}, 3 \mathrm{H}) .{ }^{13} \mathrm{C}\left\{{ }^{1} \mathrm{H}\right\} \mathrm{NMR}\left(126 \mathrm{MHz}, \mathrm{CDCl}_{3}\right) \delta$, ppm: 152.99, 104.97, 76.42, 34.33, 32.44, 31.54, 28.96, 28.20, 28.00, 22.65, 14.13, 8.22. HRMS (ESI) $\mathrm{m} / \mathrm{z}:[\mathrm{M}+\mathrm{H}]^{+}$calcd for $\mathrm{C}_{12} \mathrm{H}_{24} \mathrm{OS} 217.1621$, found $217.1628(\Delta=3.2 \mathrm{ppm}$ ). Anal. Calcd for $\mathrm{C}_{12} \mathrm{H}_{24} \mathrm{OS}$, \%: C: 66.61, H: 11.18, S: 14.82; found, \%: C: 66.61, H: 11.08, S: 14.65 .

3-(tert-butylthio)-2-methylbut-3-en-2-ol (30). The product was obtained as light brown oil after dry column vacuum chromatography.

${ }^{1} \mathrm{H}$ NMR $\left(600 \mathrm{MHz}, \mathrm{CDCl}_{3}\right) \delta$, ppm: $5.75(\mathrm{~s}, 1 \mathrm{H}), 5.39(\mathrm{~s}, 1 \mathrm{H}), 2.52$ (s,

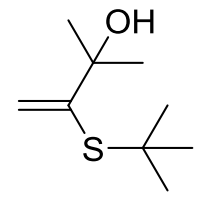
1H), 1.38 (s, 9H), 1.38 (s, 6H). ${ }^{13} \mathrm{C}\left\{{ }^{1} \mathrm{H}\right\}$ NMR (126 MHz, $\left.\mathrm{CDCl}_{3}\right) \delta$, ppm: 151.51, 117.97, 73.15, 46.73, 31.68, 29.51. HRMS (ESI) $\mathrm{m} / z$ : $[\mathrm{M}+\mathrm{Na}]^{+}$calcd for $\mathrm{C}_{9} \mathrm{H}_{18} \mathrm{OS} 197.0971$, found $197.0972(\Delta=0.5 \mathrm{ppm})$. 
3-(sec-butylthio)-2-methylbut-3-en-2-ol (3p). The reaction was conducted without $\mathrm{Y}$-terpinene. The product was obtained as yellow oil after drying under vacuum.

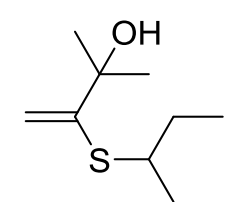

${ }^{1} \mathrm{H}$ NMR $\left(500 \mathrm{MHz}, \mathrm{CDCl}_{3}\right) \delta$, ppm: $5.49(\mathrm{~s}, 1 \mathrm{H}), 4.90(\mathrm{~s}, 1 \mathrm{H}), 3.07-2.98(\mathrm{~m}, 1 \mathrm{H})$, 2.11 (br. s, 1H), 1.78- $1.50(\mathrm{~m}, 2 \mathrm{H}), 1.44(\mathrm{~s}, 6 \mathrm{H}), 1.31(\mathrm{~d}, \mathrm{~J}=6.7 \mathrm{~Hz}, 3 \mathrm{H}), 1.00(\mathrm{t}, \mathrm{J}=$ $7.3 \mathrm{~Hz}, 3 \mathrm{H}) .{ }^{13} \mathrm{C}\left\{{ }^{1} \mathrm{H}\right\} \mathrm{NMR}\left(126 \mathrm{MHz}, \mathrm{CDCl}_{3}\right) \delta, \mathrm{ppm}: 153.34,107.34,73.84,43.06,29.75$, 29.27, 19.99, 11.60. HRMS (ESI) $\mathrm{m} / z$ : $[\mathrm{M}+\mathrm{Na}]^{+}$calcd for $\mathrm{C}_{9} \mathrm{H}_{18} \mathrm{OS} 197.0971$, found $197.0961(\Delta=5.1 \mathrm{ppm})$.

3-(cyclohexylthio)-2-methylbut-3-en-2-ol (3q). The product was obtained as yellow oil after dry column vacuum. Spectral data are in agreement with previously reported. ${ }^{9}$

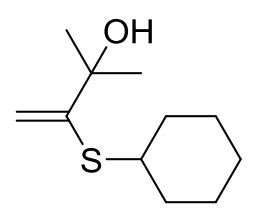

${ }^{1} \mathrm{H}$ NMR $\left(500 \mathrm{MHz}, \mathrm{CDCl}_{3}\right) \delta$, ppm: $5.48(\mathrm{~s}, 1 \mathrm{H}), 4.94(\mathrm{~s}, 1 \mathrm{H}), 3.01-2.93(\mathrm{~m}, 1 \mathrm{H})$, 2.23-2.17 (br. s, $1 \mathrm{H}), 2.10-2.00(\mathrm{~m}, 1 \mathrm{H}), 1.82-1.74(\mathrm{~m}, 2 \mathrm{H}), 1.67-1.59(\mathrm{~m}, 1 \mathrm{H}), 1.42(\mathrm{~s}$, $6 \mathrm{H}), 1.40-1.27(\mathrm{~m}, 5 \mathrm{H})$.

3-(hexylthio)-2-methylbut-3-en-2-ol (3r). The product was obtained as yellow oil after dry column vacuum chromatography.

${ }^{1} \mathrm{H}$ NMR $\left(500 \mathrm{MHz}, \mathrm{CDCl}_{3}\right)$ ס, ppm: $5.37(\mathrm{~s}, 1 \mathrm{H}), 4.76(\mathrm{~s}, 1 \mathrm{H}), 2.70$

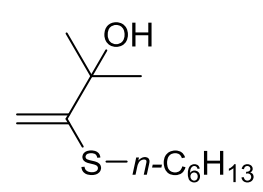
(t, J = 7.4 Hz, 2H), 2.02 (br. s, 1H), $1.77-1.59(\mathrm{~m}, 2 \mathrm{H}), 1.45(\mathrm{~s}, 6 \mathrm{H}), 1.45-1.36(\mathrm{~m}, 2 \mathrm{H})$, 1.34-1.27 (m, 4H), $0.88(\mathrm{t}, \mathrm{J}=6.9 \mathrm{~Hz}, 3 \mathrm{H}) .{ }^{13} \mathrm{C}\left\{{ }^{1} \mathrm{H}\right\} \mathrm{NMR}\left(126 \mathrm{MHz}, \mathrm{CDCl}_{3}\right) \delta$, ppm: $154.67,104.33,73.90,32.59,31.53,29.96,28.95,28.21,22.64,14.12$. HRMS (ESI) $\mathrm{m} / \mathrm{z}$ : $[\mathrm{M}+\mathrm{Na}]^{+}$calcd for $\mathrm{C}_{11} \mathrm{H}_{22} \mathrm{OS} 225.1284$, found $225.1280(\Delta=1.8 \mathrm{ppm})$.

3,3'-(pentane-1,5-diylbis(sulfanediyl))bis(2-methylbut-3-en2-ol) (3s). The product was obtained as yellow oil after drying under vacuum.

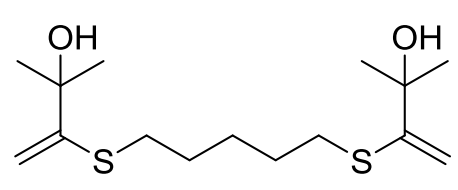

${ }^{1} \mathrm{H}$ NMR (600 MHz, $\mathrm{CDCl}_{3}$ ) $\delta$, ppm: 5.35 (s, 2H), $4.72(\mathrm{~s}, 2 \mathrm{H}), 2.68$ (t, $J=7.3 \mathrm{~Hz}$, $4 \mathrm{H}), 2.11(\mathrm{~s}, 2 \mathrm{H}), 1.71-1.62(\mathrm{~m}, 4 \mathrm{H}), 1.58-1.51(\mathrm{~m}, 2 \mathrm{H}), 1.42(\mathrm{~s}, 12 \mathrm{H}) .{ }^{13} \mathrm{C}\left\{{ }^{1} \mathrm{H}\right\}$ NMR (151 MHz, $\mathrm{CDCl}_{3}$ ) $\delta$, ppm: 154.35, 104.37, 73.83, 32.18, 29.89, 28.57, 27.70. HRMS (ESI) $\mathrm{m} / \mathrm{z}:[\mathrm{M}+\mathrm{Na}]^{+}$calcd for $\mathrm{C}_{15} \mathrm{H}_{28} \mathrm{O}_{2} \mathrm{~S}_{2} 327.1423$, found $327.1442(\Delta=5.8 \mathrm{ppm})$.

3,3'-((oxybis(ethane-2,1-diyl))bis(sulfanediyl))bis(2methylbut-3-en-2-ol) (3t). The product was obtained as yellow oil after drying under vacuum.

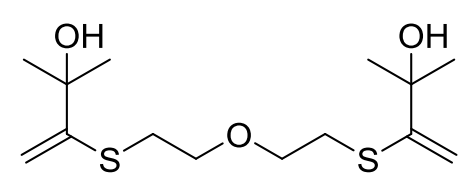

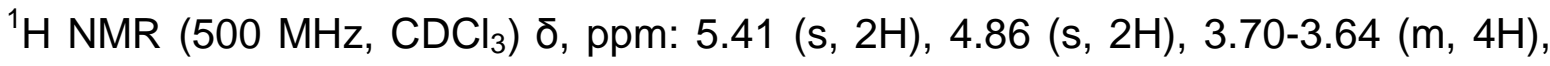
2.96-2.90 (m, 4H), 2.20 (br. s, 1H), 2.16 (br. s, 1H), 1.44 (s, 12H). ${ }^{13} \mathrm{C}\left\{{ }^{1} \mathrm{H}\right\} \mathrm{NMR}(126 \mathrm{MHz}$, $\left.\mathrm{CDCl}_{3}\right) \delta 153.65,105.94,73.84,68.80,32.23,29.83$. HRMS (ESI) $\mathrm{m} / \mathrm{z}:[\mathrm{M}+\mathrm{Na}]^{+}$calcd for $\mathrm{C}_{14} \mathrm{H}_{26} \mathrm{O}_{3} \mathrm{~S}_{2} 329.1216$, found $329.1222(\Delta=2.4 \mathrm{ppm})$. 
2-methyl-3-(propylthio)but-3-en-2-ol (3u). The product was obtained as light yellow oil after dry column vacuum chromatography.

${ }^{1} \mathrm{H}$ NMR $\left(500 \mathrm{MHz}, \mathrm{CDCl}_{3}\right) \delta$, ppm: $5.36(\mathrm{~s}, 1 \mathrm{H}), 4.76(\mathrm{~s}, 1 \mathrm{H}), 2.68$

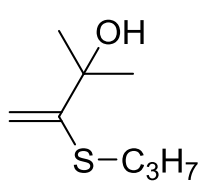
(t, J = 7.3 Hz, 2H), 1.99 (br. s, 1H), $1.73-1.64(\mathrm{~m}, 2 \mathrm{H}), 1.44(\mathrm{~s}, 6 \mathrm{H}), 1.02$ (t, J = 7.4 Hz, 3H). ${ }^{13} \mathrm{C}\left\{{ }^{1} \mathrm{H}\right\} \mathrm{NMR}\left(126 \mathrm{MHz}, \mathrm{CDCl}_{3}\right) \delta$, ppm: 154.57, 104.44, 73.90, 34.57, 29.94, 21.66, 13.83. HRMS (ESI) $\mathrm{m} / z$ : $[\mathrm{M}+\mathrm{H}]^{+}$calcd for $\mathrm{C}_{8} \mathrm{H}_{16} \mathrm{OS} 161.0995$, found $161.1010(\Delta=3.1$ ppm).

3-(ethylthio)-2-methylbut-3-en-2-ol (3v). The reaction was carried out without $y$-terpinene. The product was obtained as light yellow oil after drying under vacuum.

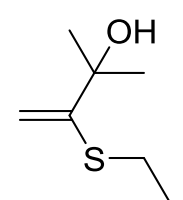

${ }^{1} \mathrm{H}$ NMR (500 MHz, $\mathrm{CDCl}_{3}$ ) $\delta$, ppm: $5.39(\mathrm{~s}, 1 \mathrm{H}), 4.78(\mathrm{~s}, 1 \mathrm{H}), 2.73$ (q, J = $7.4 \mathrm{~Hz}$, 2H), 2.02 (br. s, 1H), 1.45 (s, 6H), 1.32 (t, J = 7.4 Hz, 3H). ${ }^{13} \mathrm{C}\left\{{ }^{1} \mathrm{H}\right\} \mathrm{NMR}\left(151 \mathrm{MHz}, \mathrm{CDCl}_{3}\right)$ $\delta$, ppm: 154.30, 104.50, 73.82, 29.92, 26.46, 13.25. HRMS (ESI) $\mathrm{m} / \mathrm{z}:[\mathrm{M}+\mathrm{H}]^{+}$calcd for $\mathrm{C}_{7} \mathrm{H}_{14} \mathrm{OS} 147.0838$, found $147.0830(\Delta=5.4 \mathrm{ppm})$.

3-((2-ethylhexyl)thio)-2-methylbut-3-en-2-ol (3w). The product was obtained as light yellow oil after dry column vacuum chromatography.

${ }^{1} \mathrm{H}$ NMR $\left(500 \mathrm{MHz}, \mathrm{CDCl}_{3}\right) \delta$, ppm: $5.36(\mathrm{~s}, 1 \mathrm{H}), 4.76(\mathrm{~s}, 1 \mathrm{H})$, $2.66(\mathrm{~d}, \mathrm{~J}=6.3 \mathrm{~Hz}, 2 \mathrm{H}), 1.99$ (br. s, $1 \mathrm{H}), 1.66-1.54(\mathrm{~m}, 1 \mathrm{H}), 1.49-$

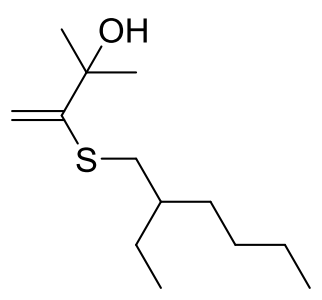
$1.34(\mathrm{~m}, 10 \mathrm{H}), 1.34-1.23(\mathrm{~m}, 4 \mathrm{H}), 0.92-0.86(\mathrm{~m}, 6 \mathrm{H}) .{ }^{13} \mathrm{C}\left\{{ }^{1} \mathrm{H}\right\} \mathrm{NMR}\left(126 \mathrm{MHz}, \mathrm{CDCl}_{3}\right) \delta$, ppm: 155.25, 104.32, 74.02, 38.43, 37.14, 32.90, 29.98, 28.99, 26.09, 23.08, 14.19, 10.93. HRMS (ESI) $\mathrm{m} / \mathrm{z}$ : $[\mathrm{M}+\mathrm{Na}]^{+}$calcd for $\mathrm{C}_{13} \mathrm{H}_{26} \mathrm{OS} 253.1597$, found $253.1607(\Delta=4.0$ ppm).

3-(isopentylthio)-2-methylbut-3-en-2-ol (3x). The product was obtained as light yellow oil after dry column vacuum chromatography.

${ }^{1} \mathrm{H}$ NMR $\left(500 \mathrm{MHz}, \mathrm{CDCl}_{3}\right) \delta$, ppm: $5.37(\mathrm{~s}, 1 \mathrm{H}), 4.76(\mathrm{~s}, 1 \mathrm{H}), 2.70(\mathrm{t}$, $\mathrm{J}=7.8 \mathrm{~Hz}, 2 \mathrm{H}), 1.97$ (br. s, 1H), $1.75-1.66(\mathrm{~m}, 1 \mathrm{H}), 1.54(\mathrm{~m}, 2 \mathrm{H}), 1.44(\mathrm{~s}$,

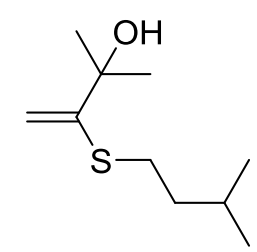
$6 \mathrm{H}), 0.92(\mathrm{~d}, \mathrm{~J}=6.6 \mathrm{~Hz}, 6 \mathrm{H}) .{ }^{13} \mathrm{C}\left\{{ }^{1} \mathrm{H}\right\} \mathrm{NMR}\left(126 \mathrm{MHz}, \mathrm{CDCl}_{3}\right) \delta$, ppm: 154.69, 104.32, 73.89, 37.13, 30.59, 29.97, 27.87, 22.40. HRMS (ESI) $\mathrm{m} / \mathrm{z}$ : $[\mathrm{M}+\mathrm{H}]^{+}$calcd for $\mathrm{C}_{10} \mathrm{H}_{20} \mathrm{OS}$ 189.1308, found 189.1305 ( $\Delta=1.6 \mathrm{ppm}$ ). Anal. Calcd for $\mathrm{C}_{10} \mathrm{H}_{20} \mathrm{OS}, \%$ : C: $63.78, \mathrm{H}$ : 10.70, S: 17.02; found, \%: C: 63.33, H: 10.64, S: 17.22.

2-methyl-3-(phenylthio)but-3-en-2-ol (3y). The product was obtained as brown oil after dry column vacuum chromatography. Spectral data are in agreement with previously reported. ${ }^{10}$

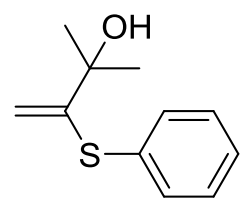


${ }^{1} \mathrm{H}$ NMR (270 MHz, $\left.\mathrm{CDCl}_{3}\right) \delta$, ppm: $7.47(\mathrm{~d}, \mathrm{~J}=7.3 \mathrm{~Hz}, 2 \mathrm{H}), 7.33(\mathrm{~m}, 3 \mathrm{H}), 5.47$ (s, $1 \mathrm{H}), 4.73$ (s, 1H), 2.06 (br. s, 1H), 1.51 (s, 6H).

3-(benzylthio)-2-methylbut-3-en-2-ol (3z). The product was obtained as orange oil after dry column vacuum chromatography. Spectral data are in agreement with previously reported. ${ }^{9}$

${ }^{1} \mathrm{H}$ NMR $\left(500 \mathrm{MHz} \mathrm{CDCl}_{3}\right) \delta$, ppm: 7.37-7.32 (m, 2H), 7.32-7.26 (m, 2H), 7.26-7.20 (m, 1H), $5.38(\mathrm{~s}, 1 \mathrm{H}), 4.81(\mathrm{~s}, 1 \mathrm{H}), 3.89$ (s, 2H), 2.26

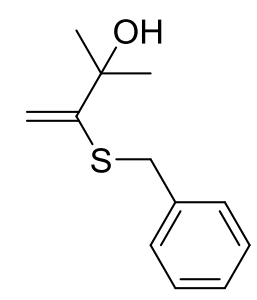
(br. s., 1H), 1.42 (s, 6H).

2-methyl-3-(phenylselanyl)but-3-en-2-ol (3aa) The product was obtained after drying under vacuum. Spectral data are in agreement with previously reported. ${ }^{11}$

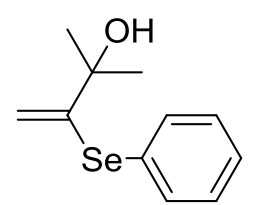

${ }^{1} \mathrm{H}$ NMR (500 MHz, $\mathrm{CDCl}_{3}$ ) $\delta$, ppm: 7.61-7.57 (m, 2H), 7.33-7.29 (m, 3H), 5.72 (s, $1 \mathrm{H}), 4.93(\mathrm{~s}, 1 \mathrm{H}), 2.28(\mathrm{~s}, 1 \mathrm{H}), 1.51(\mathrm{~s}, 6 \mathrm{H})$.

3-((2-methoxyphenyl)thio)-2-methylbut-3-en-2-ol (3ab) The product was obtained after drying under vacuum.

${ }^{1} \mathrm{H}$ NMR (500 MHz, $\mathrm{CDCl}_{3}$ ) $\delta$, ppm: 7.47 (dd, $\left.J=7.6,1.6 \mathrm{~Hz}, 1 \mathrm{H}\right), 7.31$ $(\mathrm{td}, \mathrm{J}=8.2,1.6 \mathrm{~Hz}, 1 \mathrm{H}), 6.94(\mathrm{td}, \mathrm{J}=7.5,1.0 \mathrm{~Hz}, 1 \mathrm{H}), 6.91(\mathrm{~d}, \mathrm{~J}=8.2 \mathrm{~Hz}$,

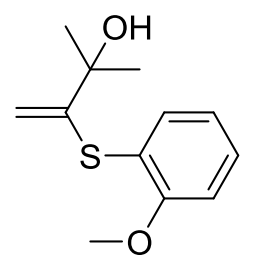
$1 \mathrm{H}), 5.43(\mathrm{~s}, 1 \mathrm{H}), 4.72(\mathrm{~s}, 1 \mathrm{H}), 3.85(\mathrm{~s}, 3 \mathrm{H}), 2.34(\mathrm{~s}, 1 \mathrm{H}), 1.62(\mathrm{~s}, 6 \mathrm{H}) .{ }^{13} \mathrm{C}\left\{{ }^{1} \mathrm{H}\right\}$ NMR $(126$ $\left.\mathrm{MHz}, \mathrm{CDCl}_{3}\right) \delta$, ppm: 158.75, 153.36, 135.42, 129.99, 122.05, 121.36, 111.48, 111.13, 73.92, 56.06, 29.55. HRMS (ESI) $\mathrm{m} / \mathrm{z}$ : $[\mathrm{M}+\mathrm{Na}]^{+}$calcd for $\mathrm{C}_{12} \mathrm{H}_{16} \mathrm{O}_{2} \mathrm{~S}$ 247.0763, found $247.0771(\Delta=3.2 \mathrm{ppm})$.

3-((3-methoxyphenyl)thio)-2-methylbut-3-en-2-ol (3ac). The product was obtained after drying under vacuum.

${ }^{1} \mathrm{H}$ NMR $\left(500 \mathrm{MHz}, \mathrm{CDCl}_{3}\right) \delta$, ppm: $7.25(\mathrm{t}, J=8.0 \mathrm{~Hz}, 1 \mathrm{H}), 7.05$

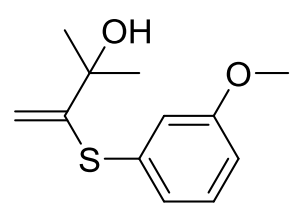
(d, $J=7.7 \mathrm{~Hz}, 1 \mathrm{H}), 7.03-7.00(\mathrm{~m}, 1 \mathrm{H}), 6.83$ (ddd, $J=8.3,2.5,0.7 \mathrm{~Hz}, 1 \mathrm{H}), 5.51(\mathrm{~s}, 1 \mathrm{H}$ ), 4.85 (s, $1 \mathrm{H}), 3.80$ (s, 3H), 2.09 (s, $1 \mathrm{H}), 1.51$ (s, 6H). ${ }^{13} \mathrm{C}\left\{{ }^{1} \mathrm{H}\right\} \mathrm{NMR}\left(126 \mathrm{MHz}^{\mathrm{CDCl}}\right)_{3} \delta$, ppm: 160.19, 154.29, 135.17, 130.14, 125.45, 118.38, 113.93, 111.94, 74.15, 55.45, 29.76. HRMS (ESI) $\mathrm{m} / \mathrm{z}$ : $[\mathrm{M}+\mathrm{Na}]^{+}$calcd for $\mathrm{C}_{12} \mathrm{H}_{16} \mathrm{O}_{2} \mathrm{~S} 247.0763$, found $247.0759(\Delta=1.6$ ppm).

3-((4-methoxyphenyl)thio)-2-methylbut-3-en-2-ol (3ad). The product was obtained after drying under vacuum.

${ }^{1} \mathrm{H}$ NMR $\left(500 \mathrm{MHz}, \mathrm{CDCl}_{3}\right) \delta$, ppm: $7.41(\mathrm{~d}, J=8.8 \mathrm{~Hz}, 2 \mathrm{H})$, $6.90(\mathrm{~d}, J=8.8 \mathrm{~Hz}, 2 \mathrm{H}), 5.30(\mathrm{~s}, 1 \mathrm{H}), 4.48(\mathrm{~s}, 1 \mathrm{H}), 3.82(\mathrm{~s}, 3 \mathrm{H}), 1.99$

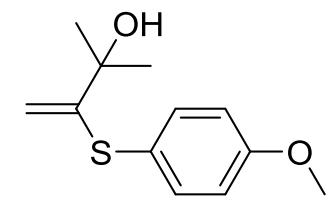


(br. s, 1H), 1.52 (s, 6H). ${ }^{13} \mathrm{C}\left\{{ }^{1} \mathrm{H}\right\}$ NMR (126 MHz, $\left.\mathrm{CDCl}_{3}\right) \delta$, ppm: 160.25, 156.85, 136.64, 123.42, 115.15, 107.29, 73.97, 55.48, 29.99. HRMS (ESI) $\mathrm{m} / \mathrm{z}:[\mathrm{M}+\mathrm{Na}]^{+}$calcd for $\mathrm{C}_{12} \mathrm{H}_{16} \mathrm{O}_{2} \mathrm{~S} 247.0763$, found 247.0776 ( $\left.\Delta=5.3 \mathrm{ppm}\right)$.

2-methyl-3-(o-tolylthio)but-3-en-2-ol (3ae). The product was obtained as light brown oil after evaporation of $\mathrm{Y}$-terpinene under vacuum.

${ }^{1} \mathrm{H}$ NMR $\left(500 \mathrm{MHz}, \mathrm{CDCl}_{3}\right) \delta$, ppm: $7.50(\mathrm{~d}, J=7.5 \mathrm{~Hz}, 1 \mathrm{H}), 7.30-$ $7.25(\mathrm{~m}, 2 \mathrm{H}), 7.22-7.16(\mathrm{~m}, 1 \mathrm{H}), 5.26(\mathrm{~s}, 1 \mathrm{H}), 4.30(\mathrm{~s}, 1 \mathrm{H}), 2.41(\mathrm{~s}, 3 \mathrm{H})$,

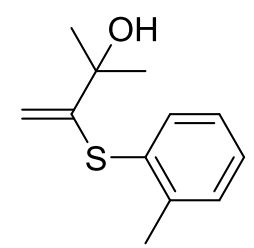
$2.00(\mathrm{~s}, 1 \mathrm{H}), 1.55$ (s, 6H). ${ }^{13} \mathrm{C}\left\{{ }^{1} \mathrm{H}\right\}$ NMR (126 MHz, $\left.\mathrm{CDCl}_{3}\right) \delta, \mathrm{ppm}: 154.24,142.16$, $136.07,131.62,130.90,129.22,126.96,106.59,74.10,29.93,20.51$. HRMS (ESI) $\mathrm{m} / \mathrm{z}$ : $[\mathrm{M}+\mathrm{Na}]^{+}$calcd for $\mathrm{C}_{12} \mathrm{H}_{16} \mathrm{OS} 231.0814$, found $231.0810(\Delta=1.7 \mathrm{ppm})$.

2-methyl-3-(p-tolylthio)but-3-en-2-ol (3af). The product was obtained as light brown oil after drying under vacuum. Spectral data are in agreement with previously reported. ${ }^{12}$

${ }^{1} \mathrm{H}$ NMR $\left(500 \mathrm{MHz}, \mathrm{CDCl}_{3}\right) \delta 7.37(\mathrm{~d}, J=7.9 \mathrm{~Hz}, 2 \mathrm{H}), 7.17(\mathrm{~d}, J=$

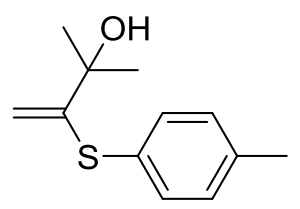
$7.9 \mathrm{~Hz}, 2 \mathrm{H}), 5.38(\mathrm{~s}, 1 \mathrm{H}), 4.63(\mathrm{~s}, 1 \mathrm{H}), 2.35(\mathrm{~s}, 3 \mathrm{H}), 2.04(\mathrm{~s}, 1 \mathrm{H}), 1.52(\mathrm{~s}, 6 \mathrm{H}) .{ }^{13} \mathrm{C} \mathrm{NMR}$ $\left(126 \mathrm{MHz}, \mathrm{CDCl}_{3}\right) \delta 155.76,138.49,134.28,130.25,129.74,109.16,74.03,29.87,21.32$.

3-((2-chlorophenyl)thio)-2-methylbut-3-en-2-ol (3ag) The product was obtained as light brown oil after drying under vacuum.

${ }^{1} \mathrm{H}$ NMR $\left(500 \mathrm{MHz}, \mathrm{CDCl}_{3}\right) \delta$, ppm: $7.59-7.52(\mathrm{~m}, 1 \mathrm{H}), 7.48-7.41$ (m, 1H), $7.27-7.21(\mathrm{~m}, 2 \mathrm{H}), 5.53(\mathrm{~s}, 1 \mathrm{H}), 4.71(\mathrm{~s}, 1 \mathrm{H}), 2.04(\mathrm{~s}, 1 \mathrm{H}), 1.53(\mathrm{~s}$,

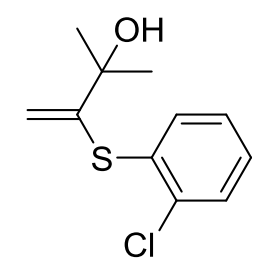
$6 \mathrm{H}) .{ }^{13} \mathrm{C}\left\{{ }^{1} \mathrm{H}\right\}$ NMR $\left(126 \mathrm{MHz}, \mathrm{CDCl}_{3}\right) \delta$, ppm: 152.26, 137.06, 134.97, 133.16, 130.36, 129.34, 127.50, 112.33, 74.24, 29.67. HRMS (ESI) $\mathrm{m} / \mathrm{z}$ : $[\mathrm{M}+\mathrm{Na}]^{+}$calcd for $\mathrm{C}_{11} \mathrm{H}_{13} \mathrm{ClOS}$ 251.0268 , found $251.0261(\Delta=2.7 \mathrm{ppm})$.

3-((3-chlorophenyl)thio)-2-methylbut-3-en-2-ol (3ah) The product was obtained as light brown oil after drying under vacuum.

${ }^{1} \mathrm{H}$ NMR $\left(500 \mathrm{MHz}, \mathrm{CDCl}_{3}\right) \delta$, ppm: $7.45(\mathrm{~s}, 1 \mathrm{H}), 7.37-7.31(\mathrm{~m}$,

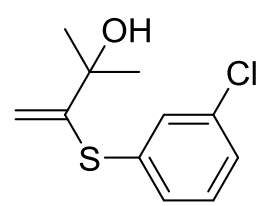
$1 \mathrm{H}), 7.27-7.23(\mathrm{~m}, 2 \mathrm{H}), 5.58(\mathrm{~s}, 1 \mathrm{H}), 4.88(\mathrm{~s}, 1 \mathrm{H}), 2.04(\mathrm{~s}, 1 \mathrm{H}), 1.50(\mathrm{~s}, 6 \mathrm{H}) .{ }^{13} \mathrm{C}\left\{{ }^{1} \mathrm{H}\right\}$ NMR (126 MHz, $\left.\mathrm{CDCl}_{3}\right) \delta$, ppm: 153.62, 136.39, 134.94, 132.39, 130.85, 130.35, 128.00 , 113.35, 74.18, 29.68. HRMS (ESI) $\mathrm{m} / z$ : $[\mathrm{M}+\mathrm{Na}]^{+}$calcd for $\mathrm{C}_{11} \mathrm{H}_{13} \mathrm{ClOS} 251.0268$, found $251.0265(\Delta=1.2 \mathrm{ppm})$.

3-((4-chlorophenyl)thio)-2-methylbut-3-en-2-ol (3ai) The product was obtained as light brown oil after drying under vacuum.

${ }^{1} \mathrm{H}$ NMR (400 MHz, $\left.\mathrm{CDCl}_{3}\right) \delta$, ppm: $7.42-7.37(\mathrm{~m}, 2 \mathrm{H}), 7.33-$

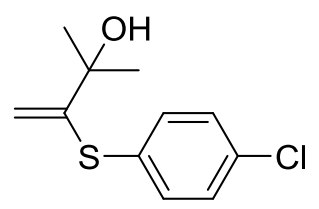
$7.28(\mathrm{~m}, 2 \mathrm{H}), 5.49(\mathrm{~d}, J=0.6 \mathrm{~Hz}, 1 \mathrm{H}), 4.74(\mathrm{~d}, J=0.6 \mathrm{~Hz}, 1 \mathrm{H}$ ), 1.97 (br. s, $1 \mathrm{H}), 1.50$ (s, $6 \mathrm{H}) .{ }^{13} \mathrm{C}\left\{{ }^{1} \mathrm{H}\right\} \operatorname{NMR}\left(101 \mathrm{MHz}, \mathrm{CDCl}_{3}\right) \delta$, ppm: 154.67, 134.78, 134.32, 132.52, 129.63, 
111.51, 74.13, 29.81. HRMS (ESI) $\mathrm{m} / \mathrm{z}$ : $[\mathrm{M}+\mathrm{Na}]^{+}$calcd for $\mathrm{C}_{11} \mathrm{H}_{13} \mathrm{ClOS} 251.0268$, found $251.0263(\Delta=2.0 \mathrm{ppm})$.

3-((4-bromophenyl)thio)-2-methylbut-3-en-2-ol (3aj). The product was obtained as light brown oil after drying under vacuum.

${ }^{1} \mathrm{H}$ NMR $\left(500 \mathrm{MHz}, \mathrm{CDCl}_{3}\right) \delta$, ppm: $7.46(\mathrm{~d}, J=8.5 \mathrm{~Hz}, 2 \mathrm{H}), 7.32$

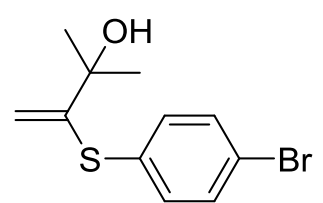
$(\mathrm{d}, J=8.5 \mathrm{~Hz}, 2 \mathrm{H}), 5.50(\mathrm{~s}, 1 \mathrm{H}), 4.77(\mathrm{~s}, 1 \mathrm{H}), 2.01(\mathrm{~s}, 1 \mathrm{H}), 1.50(\mathrm{~s}, 6 \mathrm{H}) \cdot{ }^{13} \mathrm{C}\left\{{ }^{1} \mathrm{H}\right\} \operatorname{NMR}(126$ $\mathrm{MHz}, \mathrm{CDCl}_{3}$ ) $\delta$, ppm: 154.39, 134.88, 133.23, 132.54, 122.31, 111.86, 74.12, 29.76. HRMS (ESI) $\mathrm{m} / z$ : [M+Na] ${ }^{+}$calcd for $\mathrm{C}_{11} \mathrm{H}_{13} \mathrm{BrOS} 294.9768$, found $294.9763(\Delta=1.0 \mathrm{ppm}$ ).

3-((4-fluorophenyl)thio)-2-methylbut-3-en-2-ol (3ak) The product was obtained as light brown oil after drying under vacuum.

${ }^{1} \mathrm{H}$ NMR $\left(500 \mathrm{MHz}, \mathrm{CDCl}_{3}\right) \delta$, ppm: 7.49-7.41 (m, 2H), 7.05 (t, $J=$ $8.6 \mathrm{~Hz}, 2 \mathrm{H}), 5.38(\mathrm{~s}, 1 \mathrm{H}), 4.56(\mathrm{~s}, 1 \mathrm{H}), 2.02(\mathrm{~s}, 1 \mathrm{H}), 1.51(\mathrm{~s}, 6 \mathrm{H}) .{ }^{13} \mathrm{C}\left\{{ }^{1} \mathrm{H}\right\}$

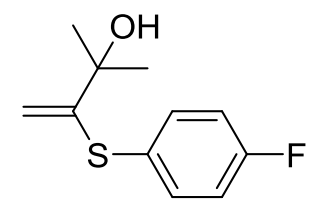
NMR $\left(126 \mathrm{MHz}, \mathrm{CDCl}_{3}\right) \delta$, ppm: $163.01\left(\mathrm{~d}, \mathrm{~J}_{\mathrm{C}-\mathrm{F}}=248.8 \mathrm{~Hz}\right.$ ), 155.81, $136.41\left(\mathrm{~d}, \mathrm{~J}_{\mathrm{C}-\mathrm{F}}=8.2\right.$ $\mathrm{Hz}), 128.50\left(\mathrm{~d}, J_{\mathrm{C}-\mathrm{F}}=0.4 \mathrm{~Hz}\right), 116.64\left(\mathrm{~d}, J_{\mathrm{C}-\mathrm{F}}=21.9 \mathrm{~Hz}\right), 109.16,74.04,29.88$. HRMS (ESI) $\mathrm{m} / z$ : $[\mathrm{M}+\mathrm{Na}]^{+}$calcd for $\mathrm{C}_{11} \mathrm{H}_{13} \mathrm{FOS} 235.0563$, found 235.0560 ( $\Delta=1.3 \mathrm{ppm}$ ).

4-((3-hydroxy-3-methylbut-1-en-2-yl)thio)phenol (3al). The product was obtained as light orange oil after drying under vacuum.

${ }^{1} \mathrm{H}$ NMR (500 MHz, Acetone- $d_{6}$ ) $\delta$, ppm: 8.66 (br. s, $1 \mathrm{H}$ ), 7.30 $(\mathrm{d}, \mathrm{J}=8.6 \mathrm{~Hz}, 2 \mathrm{H}), 6.89(\mathrm{~d}, \mathrm{~J}=8.6 \mathrm{~Hz}, 2 \mathrm{H}), 5.29(\mathrm{~s}, 1 \mathrm{H}), 4.31(\mathrm{~s}, 1 \mathrm{H})$,

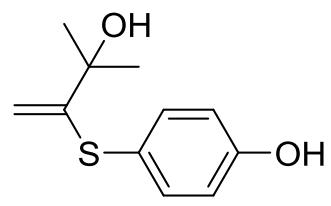
$4.00(\mathrm{~s}, 1 \mathrm{H}), 1.45(\mathrm{~s}, 6 \mathrm{H}) .{ }^{13} \mathrm{C}\left\{{ }^{1} \mathrm{H}\right\}$ NMR (126 MHz, Acetone- $\left.d_{6}\right) \delta$, ppm: 159.13, 159.04, 137.79, 122.95, 117.36, 105.65, 73.66, 30.57. HRMS (ESI) $\mathrm{m} / \mathrm{z}:[\mathrm{M}+\mathrm{Na}]^{+}$calcd for $\mathrm{C}_{11} \mathrm{H}_{14} \mathrm{O}_{2} \mathrm{~S} 233.0607$, found 233.0605 ( $\left.\Delta=0.9 \mathrm{ppm}\right)$.

3-((4-aminophenyl)thio)-2-methylbut-3-en-2-ol (3am).

${ }^{1} \mathrm{H}$ NMR $\left(400 \mathrm{MHz}, \mathrm{CDCl}_{3}\right) \delta$, ppm: $7.30-7.24(\mathrm{~m}, 2 \mathrm{H}), 6.72-$ $6.61(\mathrm{~m}, 2 \mathrm{H}), 5.24(\mathrm{~d}, J=0.5 \mathrm{~Hz}, 1 \mathrm{H}), 4.46(\mathrm{~d}, J=0.5 \mathrm{~Hz}, 1 \mathrm{H}), 1.51(\mathrm{~s}$,

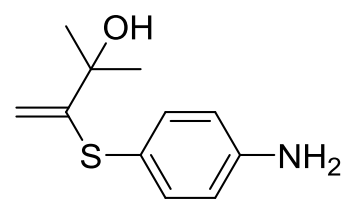
$6 \mathrm{H}) .{ }^{13} \mathrm{C}\left\{{ }^{1} \mathrm{H}\right\}$ NMR $\left(101 \mathrm{MHz}, \mathrm{CDCl}_{3}\right) \delta$, ppm: 157.42, 147.30, 136.89, 119.95, 115.99, 106.27, 73.92, 30.02. HRMS (ESI) $\mathrm{m} / z$ : $[\mathrm{M}+\mathrm{Na}]^{+}$calcd for $\mathrm{C}_{11} \mathrm{H}_{15} \mathrm{OSN} 232.0797$, found $232.0768(\Delta=0.4 \mathrm{ppm})$.

Pent-1-en-2-yl(pentyl)sulfane (3an). The product was obtained as colorless liquid after column chromatography in petroleum ether.

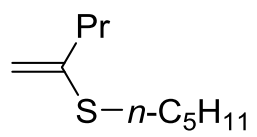

${ }^{1} \mathrm{H}$ NMR (500 MHz, $\mathrm{CDCl}_{3}$ ) $\delta$, ppm: $5.00(\mathrm{~s}, 1 \mathrm{H}), 4.68(\mathrm{~s}, 1 \mathrm{H}), 2.68$ (t, J = $7.4 \mathrm{~Hz}$, 2H), $2.19(\mathrm{t}, \mathrm{J}=7.5 \mathrm{~Hz}, 2 \mathrm{H}), 1.68-1.60(\mathrm{~m}, 2 \mathrm{H}), 1.60-1.52(\mathrm{~m}, 2 \mathrm{H}), 1.43-1.30(\mathrm{~m}$, $4 \mathrm{H}), 0.94-0.87(\mathrm{~m}, 6 \mathrm{H}) .{ }^{13} \mathrm{C}\left\{{ }^{1} \mathrm{H}\right\} \mathrm{NMR}\left(126 \mathrm{MHz}, \mathrm{CDCl}_{3}\right) \delta$, ppm: 146.01, 105.32, 39.80, $31.45,31.31,28.16,22.42,22.12,14.06,13.60$. 
2-(pentylthio)prop-2-en-1-amine (3ao). Spectral data are in agreement with previously reported. ${ }^{8}$

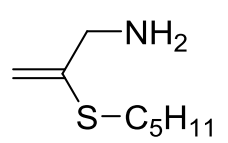

${ }^{1} \mathrm{H}$ NMR $\left(600 \mathrm{MHz}, \mathrm{C}_{6} \mathrm{D}_{6}\right) \delta$, ppm: $5.15(\mathrm{~s}, 1 \mathrm{H}), 4.74(\mathrm{~s}, 1 \mathrm{H}), 3.26(\mathrm{~s}, 2 \mathrm{H}), 2.50(\mathrm{t}$, $\mathrm{J}=7.5 \mathrm{~Hz}, 2 \mathrm{H}), 1.54-1.46(\mathrm{~m}, 2 \mathrm{H}), 1.26-1.06(\mathrm{~m}, 4 \mathrm{H}), 0.85-0.74(\mathrm{~m}, 5 \mathrm{H})$.

(1-(cyclohex-1-en-1-yl)vinyl)(pentyl)sulfane (3ap). Spectral data are in agreement with previously reported. ${ }^{7}$

${ }^{1} \mathrm{H}$ NMR $\left(600 \mathrm{MHz}, \mathrm{CDCl}_{3}\right) \delta$, ppm: $6.22(\mathrm{~m}, 1 \mathrm{H}), 5.26(\mathrm{~s}, 1 \mathrm{H}), 4.90(\mathrm{~s}$,

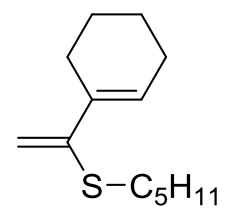
$1 \mathrm{H}), 2.68(\mathrm{t}, \mathrm{J}=7.4 \mathrm{~Hz}, 2 \mathrm{H}), 2.24(\mathrm{~m}, 2 \mathrm{H}), 2.15(\mathrm{~m}, 2 \mathrm{H}), 1.68(\mathrm{~m}, 2 \mathrm{H}), 1.64(\mathrm{~m}, 2 \mathrm{H}), 1.59$ $(\mathrm{m}, 2 \mathrm{H}), 1.40(\mathrm{~m}, 2 \mathrm{H}), 1.34(\mathrm{~m}, 2 \mathrm{H}), 0.90(\mathrm{t}, \mathrm{J}=7.2 \mathrm{~Hz}, 3 \mathrm{H}) .{ }^{13} \mathrm{C}\left\{{ }^{1} \mathrm{H}\right\} \mathrm{NMR}(151 \mathrm{MHz}$, $\left.\mathrm{CDCl}_{3}\right)$ $\delta$, ppm: 145.91, 135.64, 127.25, 106.77, 32.03, 31.41, 28.40, 27.12, 25.85, 23.02, $22.44,22.25,14.08$.

3-methoxyprop-1-en-2-yl)(pentyl)sulfane (3aq).

${ }^{1} \mathrm{H}$ NMR $\left(500 \mathrm{MHz}, \mathrm{C}_{6} \mathrm{D}_{6}\right) \delta$, ppm: $5.31(\mathrm{~s}, 1 \mathrm{H}), 4.89(\mathrm{~s}, 1 \mathrm{H}), 3.93(\mathrm{~s}$, $2 \mathrm{H}), 3.12(\mathrm{~s}, 3 \mathrm{H}), 2.51(\mathrm{t}, \mathrm{J}=7.4 \mathrm{~Hz}, 2 \mathrm{H}), 1.53-1.46(\mathrm{~m}, 2 \mathrm{H}), 1.22-1.10(\mathrm{~m}$,

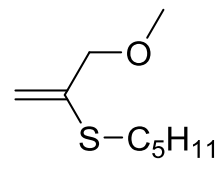
$4 \mathrm{H}), 0.79$ (t, J = 7.1 Hz, 3H). ${ }^{13} \mathrm{C}\left\{{ }^{1} \mathrm{H}\right\}$ NMR (126 MHz, $\left.\mathrm{C}_{6} \mathrm{D}_{6}\right) \delta, \mathrm{ppm}: 143.68,107.83$, 75.48, 57.50, 31.44, 31.09, 28.51, 22.56, 14.12. HRMS (ESI) $\mathrm{m} / \mathrm{z}:[\mathrm{M}+\mathrm{Na}]^{+}$calcd for $\mathrm{C}_{9} \mathrm{H}_{18} \mathrm{OS} 175.1151$, found $175.1156(\Delta=2.9 \mathrm{ppm})$.

\section{Thiophenol-d (1b- $d$ )}

Thiophenol- $d$ was obtained as a product of proton/deuterium exchange reaction of $\mathrm{PhSH}$ with $\mathrm{D}_{2} \mathrm{O}: 0.2 \mathrm{~mL}$ of $\mathrm{PhSH}$ was added to $0.4 \mathrm{~mL}$ of $\mathrm{D}_{2} \mathrm{O}$ in the screw-capped tube, the reaction was carried out in the ultrasound bath for $5 \mathrm{~h}$. Afterwards the solution was centrifuged and top layer was collected. The resting water was evaporated in vacuo and PhSD was obtained as colorless liquid. The deuterium incorporation was determined to be 95\% atom \% $D$ according to ${ }^{1} \mathrm{H}$ NMR.

\section{Preparation of (IMes)Pd(acac)Cl}

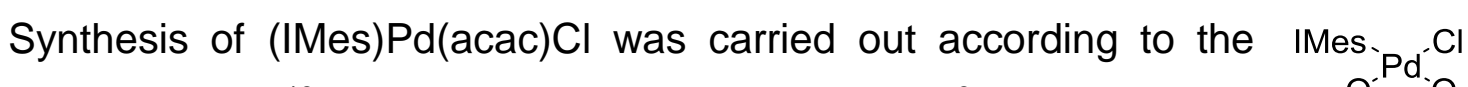
published procedure. ${ }^{13}$ Reaction of $\mathrm{Pd}(\mathrm{acac})_{2}\left(1.34 \times 10^{-3} \mathrm{~mol}, 0.408 \mathrm{~g}\right)$ with IMes $\cdot \mathrm{HCl}\left(1.47 \times 10^{-3} \mathrm{~mol}, 0.500 \mathrm{~g}\right)$ in 1,4-dioxane $(8 \mathrm{~mL})$ was carried<smiles>CC(=[Po+])C=C(C)O</smiles>
out under reflux for $24 \mathrm{~h}$. The color of reaction mixture changed from yellow to brown. 1.4dioxane was evaporated under vacuum and $\mathrm{Et}_{2} \mathrm{O}$ was added. The mixture was filtered off over the plug of Celite 545 and the solid residue was washed with $\mathrm{Et}_{2} \mathrm{O}(3 \times 5 \mathrm{~mL})$. The product was washed off from Celite with DCM $(15 \mathrm{~mL})$ affording yellow solution. DCM was 
evaporated under vacuum and $0.55 \mathrm{~g}(75 \%)$ of the complex was obtained. Spectral data are in agreement with the literature data. ${ }^{13}$

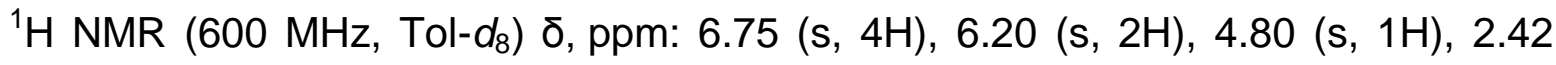
(br.s, 6H), 2.15 (br.s, 6H), 2.06 (s, 6H), $1.66(\mathrm{~s}, 3 \mathrm{H}), 1.31$ (s, 3H).

${ }^{1} \mathrm{H}$ NMR $\left(500 \mathrm{MHz}, \mathrm{C}_{6} \mathrm{D}_{6}\right) \delta$, ppm: $6.79(\mathrm{~s}, 4 \mathrm{H}), 6.17(\mathrm{~s}, 2 \mathrm{H}), 4.79(\mathrm{~s}, 1 \mathrm{H}), 2.48$ (br.s, 6H), 2.19 (br.s, 6H), 2.07 (s, 6H), 1.64 (s, 3H), 1.32 (s, 3H).

\section{Preparation of the ((IMes)Pd(SPh $\left.)_{2}\right)_{2}$ complex (Ila).}

(IMes) $\mathrm{Pd}(\mathrm{acac}) \mathrm{Cl}\left(3.6 \times 10^{-5} \mathrm{~mol}, 0.020 \mathrm{~g}\right)$ was dissolved in $0.6 \mathrm{~mL}$ of benzene in ultrasound bath. Triethylamine $\left(3.6 \cdot 10^{-5} \mathrm{~mol}, 0.004 \mathrm{~g}\right)$ was added and solution was shaken for a couple of minutes, followed by addition of 2 equiv $\mathrm{PhSH}\left(8 \times 10^{-5} \mathrm{~mol}, 0.009 \mathrm{~g}\right)$. The reaction mixture was stirred for $3 \mathrm{~h}$ at $\mathrm{rt}$ and the solution color changed from yellow to orange. The solution was then filtered through Celite 545 with benzene as eluent. Solvent and excess of thiol were evaporated under vacuum, and bright orange powder was obtained. The residue was dissolved in a minimum amount of DCM and left in an open air. X-ray quality crystals were formed upon evaporation, the crystals were collected and washed with petroleum ether and dried under vacuum.

${ }^{1} \mathrm{H}$ NMR $\left(500 \mathrm{MHz}, \mathrm{CD}_{2} \mathrm{Cl}_{2}\right) \delta$, ppm: $7.29(\mathrm{~d}, J=7.2 \mathrm{~Hz}, 4 \mathrm{H}), 7.09-7.01(\mathrm{~m}, 4 \mathrm{H})$, 6.94 (t, $J=7.3 \mathrm{~Hz}, 2 \mathrm{H}), 6.89-6.81(\mathrm{~m}, 6 \mathrm{H}), 6.75(\mathrm{~s}, 4 \mathrm{H}), 6.72-6.56(\mathrm{~m}, 12 \mathrm{H}), 2.31$ (br. s, 12H), 2.01 (br. s, $12 \mathrm{H}$ ), 1.62 (br. s, $12 \mathrm{H}$ ). ${ }^{13} \mathrm{C}\left\{{ }^{1} \mathrm{H}\right\} \mathrm{NMR}\left(126 \mathrm{MHz}, \mathrm{CD}_{2} \mathrm{Cl}_{2}\right) \delta$, ppm: $171.13,147.93,140.13,138.63,136.30,136.22,135.95,134.92,133.06,129.68,129.30$, $127.48,126.91,124.18,124.06,121.21,21.50,19.07,18.44$. HRMS (ESI) $\mathrm{m} / \mathrm{z}$ : [M] ${ }^{+}$calcd for $\mathrm{C}_{60} \mathrm{H}_{63} \mathrm{~N}_{4} \mathrm{Pd}_{2} \mathrm{~S}_{3} 1147.2285=\left[\mathrm{C}_{66} \mathrm{H}_{68} \mathrm{~N}_{4} \mathrm{Pd}_{2} \mathrm{~S}_{4}-\mathrm{PhS}\right]$, found 1147.2289; [M] ${ }^{+}$calcd for $\mathrm{C}_{54} \mathrm{H}_{57} \mathrm{~N}_{4} \mathrm{Pd}_{2} \mathrm{~S}_{2} 1038.2173=\left[\mathrm{C}_{66} \mathrm{H}_{68} \mathrm{~N}_{4} \mathrm{Pd}_{2} \mathrm{~S}_{4}-2 \mathrm{PhS}\right]$, found 1038.2178. 


\section{S9. X-Ray Crystal Structure Determination of Ila}

Data were collected on a Bruker SMART APEX-II CCD diffractometer $\left(\lambda\left(\mathrm{MoK}_{\alpha}\right)\right.$ radiation, graphite monochromator, $\omega$ and $\varphi$ scan mode) and corrected for absorption using the SADABS program. ${ }^{16}$ The structure was solved by direct methods and refined by full-matrix least squares technique on $F^{2}$ with anisotropic displacement parameters for non-hydrogen atoms. All hydrogen atoms were placed in calculated positions and refined within the riding model with fixed isotropic displacement parameters $\left(U_{\text {iso }}(H)=1.5 U_{\text {eq }}(C)\right.$ for the $\mathrm{CH}_{3}$-groups and $U_{\text {iso }}(\mathrm{H})=1.2 U_{\text {eq }}(\mathrm{C})$ for the other groups). All calculations were carried out using the SHELXTL program. ${ }^{17}$ Crystallographic data for lla have been deposited with the Cambridge Crystallographic Data Center. CCDC 1415292 contains the supplementary crystallographic data for this paper. These data can be obtained free of charge from the Director, CCDC, 12 Union Road, Cambridge CB2 1EZ, UK (Fax: +44 1223 336033; e-mail: deposit@ccdc.cam.ac.uk or www.ccdc.cam.ac.uk).

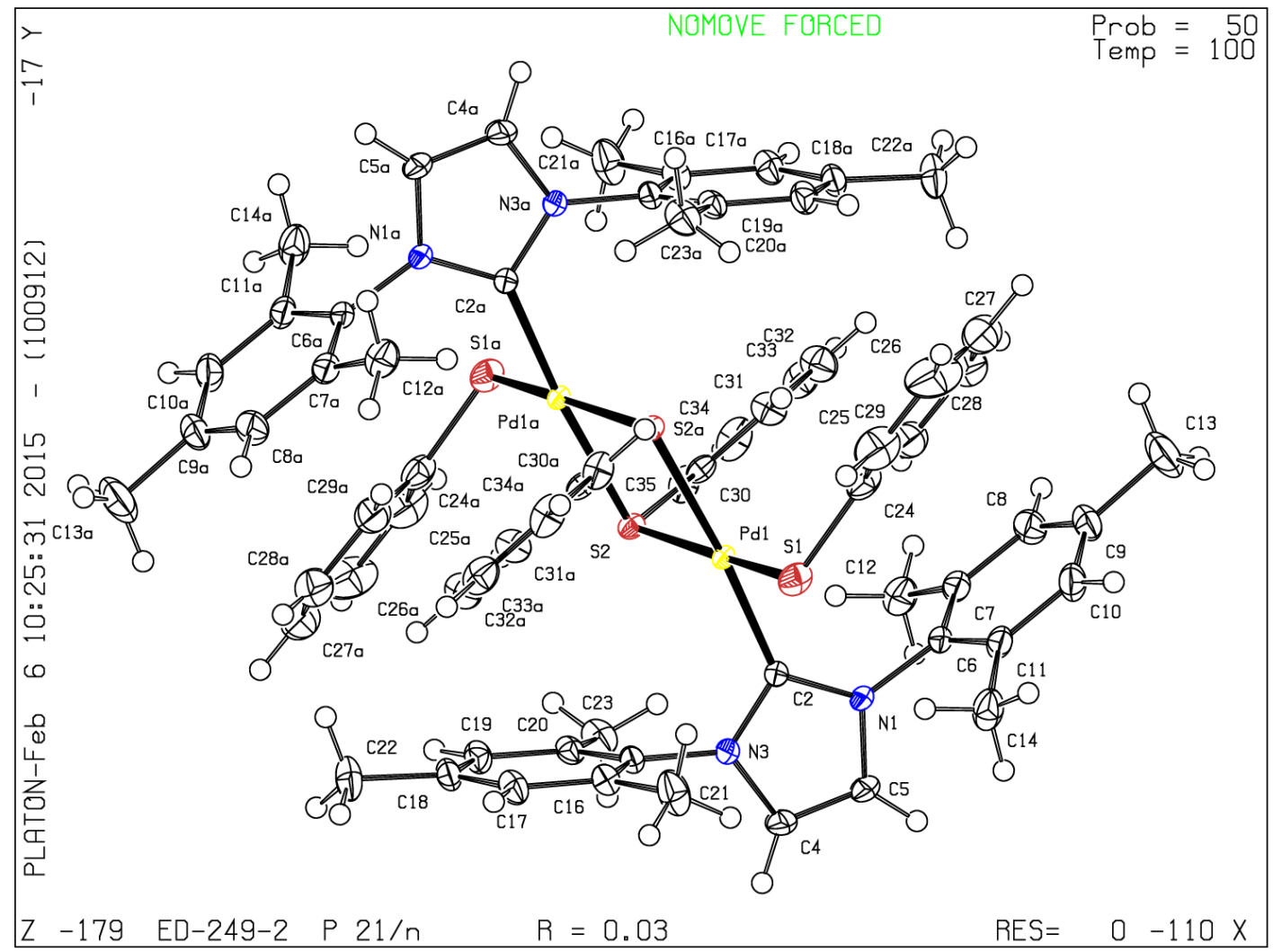


Table S3. Crystal data and structure refinement for Ila.

Identification code Ila

Empirical formula $\mathrm{C} 66 \mathrm{H} 68 \mathrm{~N} 4 \mathrm{Pd} 2 \mathrm{~S} 4$

Formula weight $\quad 1258.28$

Temperature $\quad 100(2) \mathrm{K}$

Wavelength $\quad 0.71073 \AA$

Crystal system Monoclinic

Space group $\quad P 2_{1} / \mathrm{n}$

Unit cell dimensions $a=15.6832(7) \AA \alpha=90^{\circ}$.

$$
\begin{array}{ll}
\mathrm{b}=11.3541(5) \AA & \beta=92.1890(10)^{\circ} . \\
\mathrm{c}=16.7870(7) \AA & \gamma=90^{\circ} .
\end{array}
$$

Volume 2987.1(2) $\AA^{3}$

Z 2

Density (calculated) $1.399 \mathrm{Mg} / \mathrm{m}^{3}$

Absorption coefficient $0.785 \mathrm{~mm}^{-1}$

$\mathrm{F}(000) 1296$

Crystal size $\quad 0.30 \times 0.20 \times 0.20 \mathrm{~mm}^{3}$

Theta range for data collection 1.744 to $32.709^{\circ}$.

Index ranges $\quad-23<=\mathrm{h}<=23,-17<=\mathrm{k}<=17,-24<=\mathrm{k}<=25$

Reflections collected $\quad 46245$

Independent reflections $10982[\mathrm{R}(\mathrm{int})=0.0518]$

Completeness to theta $=25.242^{\circ} 100.0 \%$

Absorption correction Semi-empirical from equivalents

Max. and min. transmission 0.855 and 0.778

Refinement method Full-matrix least-squares on $\mathrm{F}^{2}$

Data / restraints / parameters 10982 / 0 / 349

Goodness-of-fit on $\mathrm{F}^{2} \quad 1.021$

Final $R$ indices [for 8964 rflns with $I>2 \sigma(I)] \quad R 1=0.0349$, wR2 $=0.0759$

$R$ indices (all data) $R 1=0.0479, w R 2=0.0818$

Extinction coefficient $\quad \mathrm{n} / \mathrm{a}$

Largest diff. peak and hole 1.051 and -0.534 e. $\AA^{-3}$ 
Table S4. Atomic coordinates $\left(\times 10^{4}\right)$ and equivalent isotropic displacement parameters $\left(\AA^{2} \times 10^{3}\right)$

for Ila. $\mathrm{U}(\mathrm{eq})$ is defined as one third of the trace of the orthogonalized Uij tensor.

\begin{tabular}{llll}
\hline Atom $x$ & $y$ & $z$ & $U(e q)$
\end{tabular}

\begin{tabular}{|c|c|c|c|c|}
\hline $\mathrm{Pd}(1)$ & $4139(1)$ & $4717(1)$ & $4331(1)$ & $12(1)$ \\
\hline$S(1)$ & $3090(1)$ & 5974(1) & $3816(1)$ & $23(1)$ \\
\hline$S(2)$ & $5336(1)$ & $3716(1)$ & 4863(1) & $15(1)$ \\
\hline$N(1)$ & 3061(1) & 2714(1) & $3524(1)$ & $18(1)$ \\
\hline$C(2)$ & $3759(1)$ & $3428(2)$ & $3566(1)$ & $16(1)$ \\
\hline$N(3)$ & 4193(1) & $3132(1)$ & 2909(1) & $18(1)$ \\
\hline C(4) & $3774(1)$ & $2257(2)$ & 2469(1) & $27(1)$ \\
\hline$C(5)$ & $3069(1)$ & 1997(2) & $2856(1)$ & $28(1)$ \\
\hline C(6) & $2387(1)$ & 2697(2) & 4083(1) & $19(1)$ \\
\hline C(7) & 2484(1) & 1992(2) & $4760(1)$ & $22(1)$ \\
\hline C(8) & 1841(1) & 2042(2) & $5310(1)$ & $28(1)$ \\
\hline C(9) & 1118(1) & $2728(2)$ & 5182(1) & $30(1)$ \\
\hline$C(10)$ & $1018(1)$ & 3338(2) & 4472(1) & $27(1)$ \\
\hline$C(11)$ & $1643(1)$ & 3332(2) & $3904(1)$ & $23(1)$ \\
\hline$C(12)$ & $3227(1)$ & $1159(2)$ & 4871(1) & $29(1)$ \\
\hline$C(13)$ & $448(2)$ & $2807(3)$ & 5806(2) & $48(1)$ \\
\hline$C(14)$ & $1498(1)$ & 3954(2) & $3119(1)$ & $33(1)$ \\
\hline$C(15)$ & $4984(1)$ & $3671(2)$ & $2690(1)$ & $18(1)$ \\
\hline$C(16)$ & $4951(1)$ & $4707(2)$ & 2240(1) & $21(1)$ \\
\hline$C(17)$ & $5726(1)$ & $5200(2)$ & $2037(1)$ & $24(1)$ \\
\hline C(18) & $6504(1)$ & $4700(2)$ & $2276(1)$ & $22(1)$ \\
\hline C(19) & 6502(1) & $3654(2)$ & 2704(1) & $22(1)$ \\
\hline$C(20)$ & $5745(1)$ & 3111(2) & 2913(1) & $19(1)$ \\
\hline C(21) & $4124(1)$ & 5281(2) & 1980(2) & $35(1)$ \\
\hline C(22) & $7331(1)$ & $5290(2)$ & 2082(2) & $33(1)$ \\
\hline C(23) & $5768(1)$ & 1936(2) & 3330(1) & $27(1)$ \\
\hline C(24) & 2472(1) & $6354(2)$ & 4634(1) & $28(1)$ \\
\hline C(25) & 2112(2) & 7488(2) & 4670(2) & $40(1)$ \\
\hline$C(26)$ & $1595(2)$ & $7800(3)$ & $5267(2)$ & $55(1)$ \\
\hline C(27) & 1394(2) & 6954(3) & 5879(2) & $52(1)$ \\
\hline C(28) & $1745(2)$ & 5873(3) & 5841(2) & $41(1)$ \\
\hline C(29) & $2285(1)$ & $5559(2)$ & $5235(1)$ & $32(1)$ \\
\hline$C(30)$ & 4971(1) & $2793(2)$ & 5635(1) & 18(1) \\
\hline$C(31)$ & $4318(1)$ & $3119(2)$ & 6124(1) & $24(1)$ \\
\hline C(32) & $4080(2)$ & 2376(2) & 6735(1) & $29(1)$ \\
\hline C(33) & $4494(1)$ & $1307(2)$ & 6858(1) & $29(1)$ \\
\hline C(34) & $5142(1)$ & $975(2)$ & $6373(1)$ & $29(1)$ \\
\hline C(35) & $5383(1)$ & $1715(2)$ & 5762(1) & $23(1)$ \\
\hline
\end{tabular}




$\begin{array}{ll}\mathrm{Pd}(1)-\mathrm{C}(2) & 2.0212(17) \\ \mathrm{Pd}(1)-\mathrm{S}(1) & 2.3190(5) \\ \mathrm{Pd}(1)-\mathrm{S}(2) & 2.3431(4) \\ \mathrm{Pd}(1)-\mathrm{S}(2) \# 1 & 2.3648(4) \\ \mathrm{S}(1)-\mathrm{C}(24) & 1.764(2) \\ \mathrm{S}(2)-\mathrm{C}(30) & 1.7798(19) \\ \mathrm{S}(2)-\mathrm{Pd}(1) \# 1 & 2.3649(4) \\ \mathrm{N}(1)-\mathrm{C}(2) & 1.362(2) \\ \mathrm{N}(1)-\mathrm{C}(5) & 1.386(2) \\ \mathrm{N}(1)-\mathrm{C}(6) & 1.440(2) \\ \mathrm{C}(2)-\mathrm{N}(3) & 1.360(2) \\ \mathrm{N}(3)-\mathrm{C}(4) & 1.389(2) \\ \mathrm{N}(3)-\mathrm{C}(15) & 1.443(2) \\ \mathrm{C}(4)-\mathrm{C}(5) & 1.338(3) \\ \mathrm{C}(4)-\mathrm{H}(4) & 0.9500 \\ \mathrm{C}(5)-\mathrm{H}(5) & 0.9500 \\ \mathrm{C}(6)-\mathrm{C}(7) & 1.394(3) \\ \mathrm{C}(6)-\mathrm{C}(11) & 1.395(3) \\ \mathrm{C}(7)-\mathrm{C}(8) & 1.395(3) \\ \mathrm{C}(7)-\mathrm{C}(12) & 1.507(3) \\ \mathrm{C}(8)-\mathrm{C}(9) & 1.385(3) \\ \mathrm{C}(8)-\mathrm{H}(8) & 0.9500 \\ \mathrm{C}(9)-\mathrm{C}(10) & 1.382(3) \\ \mathrm{C}(9)-\mathrm{C}(13) & 1.514(3) \\ \mathrm{C}(10)-\mathrm{C}(11) & 1.394(3) \\ \mathrm{C}(10)-\mathrm{H}(10) & 0.9500 \\ \mathrm{C}(11)-\mathrm{C}(14) & 1.504(3) \\ \mathrm{C}(12)-\mathrm{H}(12 \mathrm{~A}) & 0.9800 \\ \mathrm{C}(12)-\mathrm{H}(12 \mathrm{~B}) & 0.9800 \\ \mathrm{C}(12)-\mathrm{H}(12 \mathrm{C}) & 0.9800 \\ \mathrm{C}(13)-\mathrm{H}(13 \mathrm{~A}) & 0.9800 \\ \mathrm{C}(13)-\mathrm{H}(13 \mathrm{~B}) & 0.9800 \\ \mathrm{C}(13)-\mathrm{H}(13 \mathrm{C}) & 0.9800 \\ \mathrm{C}(14)-\mathrm{H}(14 \mathrm{~A}) & 0.9800 \\ \mathrm{C}(14)-\mathrm{H}(14 \mathrm{~B}) & 0.9800 \\ \mathrm{C}(14)-\mathrm{H}(14 \mathrm{C}) & 0.9800 \\ \mathrm{C}(15)-\mathrm{C}(20) & 1.391(3) \\ \mathrm{C}(15)-\mathrm{C}(16) & 1.398(3) \\ \mathrm{C}(16)-\mathrm{C}(17) & 1.391(3) \\ \mathrm{C}(16)-\mathrm{C}(21) & 1.502(3) \\ \mathrm{C}(17)-\mathrm{C}(18) & 1.392(3) \\ \mathrm{C}(17)-\mathrm{H}(17) & 0.9500 \\ \mathrm{C}(18)-\mathrm{C}(19) & 1.388(3) \\ \mathrm{C}(18)-\mathrm{C}(22) & 1.506(3) \\ \mathrm{C}(19)-\mathrm{C}(20) & 1.393(3) \\ \mathrm{C}(19)-\mathrm{H}(19) & 0.9500 \\ & \end{array}$

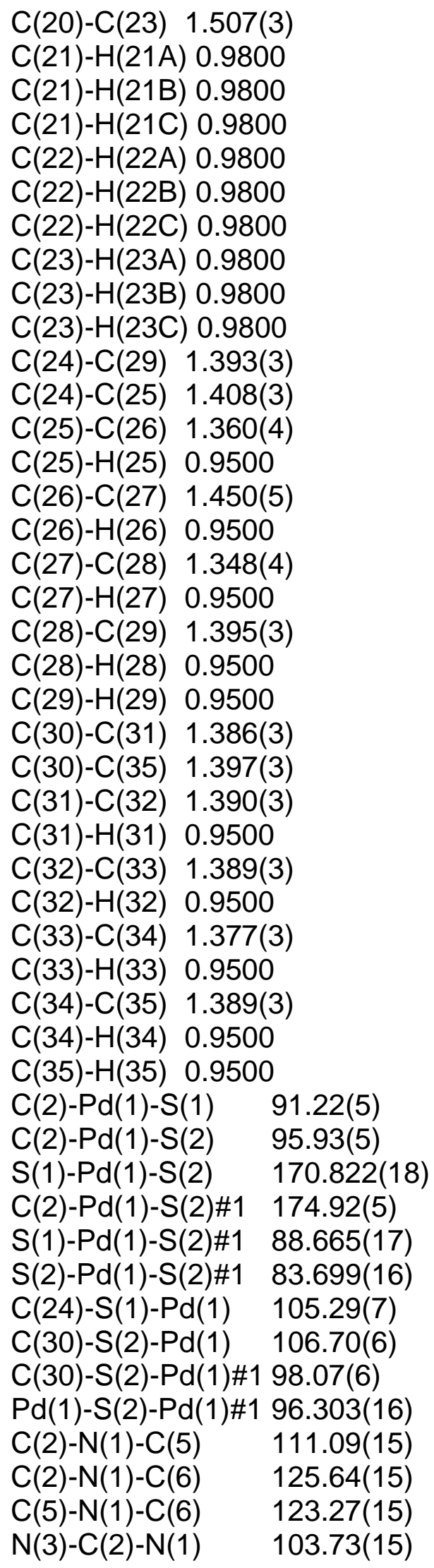




\begin{tabular}{|c|c|}
\hline$N(3)-C(2)-P d(1)$ & 123.32(12) \\
\hline$N(1)-C(2)-P d(1)$ & 132.88(13) \\
\hline$C(2)-N(3)-C(4)$ & 111.52(15) \\
\hline$C(2)-N(3)-C(15)$ & 124.37(15) \\
\hline$C(4)-N(3)-C(15)$ & 124.11(15) \\
\hline$C(5)-C(4)-N(3)$ & $106.41(17)$ \\
\hline $\mathrm{C}(5)-\mathrm{C}(4)-\mathrm{H}(4)$ & 126.8 \\
\hline $\mathrm{N}(3)-\mathrm{C}(4)-\mathrm{H}(4)$ & 126.8 \\
\hline$C(4)-C(5)-N(1)$ & $107.25(17)$ \\
\hline $\mathrm{C}(4)-\mathrm{C}(5)-\mathrm{H}(5)$ & 126.4 \\
\hline $\mathrm{N}(1)-\mathrm{C}(5)-\mathrm{H}(5)$ & 126.4 \\
\hline$C(7)-C(6)-C(11)$ & $122.45(17)$ \\
\hline$C(7)-C(6)-N(1)$ & 118.62(17) \\
\hline$C(11)-C(6)-N(1)$ & 118.83(17) \\
\hline$C(6)-C(7)-C(8)$ & 117.22(19) \\
\hline$C(6)-C(7)-C(12)$ & 121.38(18) \\
\hline$C(8)-C(7)-C(12)$ & 121.30(19) \\
\hline$C(9)-C(8)-C(7)$ & 121.93(19) \\
\hline $\mathrm{C}(9)-\mathrm{C}(8)-\mathrm{H}(8)$ & 119.0 \\
\hline $\mathrm{C}(7)-\mathrm{C}(8)-\mathrm{H}(8)$ & 119.0 \\
\hline$C(10)-C(9)-C(8)$ & 118.71(19) \\
\hline$C(10)-C(9)-C(13)$ & $120.5(2)$ \\
\hline$C(8)-C(9)-C(13)$ & $120.8(2)$ \\
\hline$C(9)-C(10)-C(11)$ & $121.9(2)$ \\
\hline $\mathrm{C}(9)-\mathrm{C}(10)-\mathrm{H}(10)$ & 119.1 \\
\hline$C(11)-C(10)-H(10)$ & 119.1 \\
\hline$C(10)-C(11)-C(6)$ & $117.37(18)$ \\
\hline$C(10)-C(11)-C(14)$ & 120.72(19) \\
\hline$C(6)-C(11)-C(14)$ & $121.88(18)$ \\
\hline $\mathrm{C}(7)-\mathrm{C}(12)-\mathrm{H}(12 \mathrm{~A})$ & 109.5 \\
\hline $\mathrm{C}(7)-\mathrm{C}(12)-\mathrm{H}(12 \mathrm{~B})$ & 109.5 \\
\hline $\mathrm{H}(12 \mathrm{~A})-\mathrm{C}(12)-\mathrm{H}(12$ & B) 109.5 \\
\hline $\mathrm{C}(7)-\mathrm{C}(12)-\mathrm{H}(12 \mathrm{C})$ & 109.5 \\
\hline$H(12 A)-C(12)-H(12$ & C) 109.5 \\
\hline $\mathrm{H}(12 \mathrm{~B})-\mathrm{C}(12)-\mathrm{H}(12$ & C) 109.5 \\
\hline $\mathrm{C}(9)-\mathrm{C}(13)-\mathrm{H}(13 \mathrm{~A})$ & 109.5 \\
\hline $\mathrm{C}(9)-\mathrm{C}(13)-\mathrm{H}(13 \mathrm{~B})$ & 109.5 \\
\hline$H(13 A)-C(13)-H(13$ & B) 109.5 \\
\hline $\mathrm{C}(9)-\mathrm{C}(13)-\mathrm{H}(13 \mathrm{C})$ & 109.5 \\
\hline$H(13 A)-C(13)-H(13$ & C) 109.5 \\
\hline $\mathrm{H}(13 \mathrm{~B})-\mathrm{C}(13)-\mathrm{H}(13$ & C) 109.5 \\
\hline$C(11)-C(14)-H(14 A$ & 109.5 \\
\hline $\mathrm{C}(11)-\mathrm{C}(14)-\mathrm{H}(14 \mathrm{~B}$ & 109.5 \\
\hline$H(14 A)-C(14)-H(14$ & B) 109.5 \\
\hline$C(11)-C(14)-H(14 C$ & 109.5 \\
\hline $\mathrm{H}(14 \mathrm{~A})-\mathrm{C}(14)-\mathrm{H}(14$ & C) 109.5 \\
\hline $\mathrm{H}(14 \mathrm{~B})-\mathrm{C}(14)-\mathrm{H}(14$ & C) 109.5 \\
\hline$C(20)-C(15)-C(16)$ & $122.90(17)$ \\
\hline$C(20)-C(15)-N(3)$ & 118.36(17) \\
\hline $\mathrm{C}(16)-\mathrm{C}(15)-\mathrm{N}(3)$ & 118.71(17) \\
\hline$C(17)-C(16)-C(15)$ & $117.15(18)$ \\
\hline$C(17)-C(16)-C(21)$ & $120.50(18)$ \\
\hline
\end{tabular}

$\mathrm{C}(15)-\mathrm{C}(16)-\mathrm{C}(21) \quad 122.36(17)$

$\mathrm{C}(16)-\mathrm{C}(17)-\mathrm{C}(18) \quad 121.98(18)$

$\mathrm{C}(16)-\mathrm{C}(17)-\mathrm{H}(17) \quad 119.0$

$\mathrm{C}(18)-\mathrm{C}(17)-\mathrm{H}(17) \quad 119.0$

C(19)-C(18)-C(17) 118.61(17)

C(19)-C(18)-C(22) 120.80(19)

$\mathrm{C}(17)-\mathrm{C}(18)-\mathrm{C}(22) \quad 120.59(19)$

C(18)-C(19)-C(20) 121.83(18)

$\mathrm{C}(18)-\mathrm{C}(19)-\mathrm{H}(19) \quad 119.1$

$\mathrm{C}(20)-\mathrm{C}(19)-\mathrm{H}(19) \quad 119.1$

C(15)-C(20)-C(19) 117.44(17)

$\mathrm{C}(15)-\mathrm{C}(20)-\mathrm{C}(23) \quad 122.28(17)$

$\mathrm{C}(19)-\mathrm{C}(20)-\mathrm{C}(23) \quad 120.22(18)$

$\mathrm{C}(16)-\mathrm{C}(21)-\mathrm{H}(21 \mathrm{~A}) 109.5$

$\mathrm{C}(16)-\mathrm{C}(21)-\mathrm{H}(21 \mathrm{~B}) 109.5$

$\mathrm{H}(21 \mathrm{~A})-\mathrm{C}(21)-\mathrm{H}(21 \mathrm{~B}) \quad 109.5$

$\mathrm{C}(16)-\mathrm{C}(21)-\mathrm{H}(21 \mathrm{C}) \quad 109.5$

$\mathrm{H}(21 \mathrm{~A})-\mathrm{C}(21)-\mathrm{H}(21 \mathrm{C}) \quad 109.5$

$\mathrm{H}(21 \mathrm{~B})-\mathrm{C}(21)-\mathrm{H}(21 \mathrm{C}) \quad 109.5$

$\mathrm{C}(18)-\mathrm{C}(22)-\mathrm{H}(22 \mathrm{~A}) \quad 109.5$

$\mathrm{C}(18)-\mathrm{C}(22)-\mathrm{H}(22 \mathrm{~B}) \quad 109.5$

$\mathrm{H}(22 \mathrm{~A})-\mathrm{C}(22)-\mathrm{H}(22 \mathrm{~B}) \quad 109.5$

$\mathrm{C}(18)-\mathrm{C}(22)-\mathrm{H}(22 \mathrm{C}) \quad 109.5$

$\mathrm{H}(22 \mathrm{~A})-\mathrm{C}(22)-\mathrm{H}(22 \mathrm{C}) \quad 109.5$

$\mathrm{H}(22 \mathrm{~B})-\mathrm{C}(22)-\mathrm{H}(22 \mathrm{C}) \quad 109.5$

$\mathrm{C}(20)-\mathrm{C}(23)-\mathrm{H}(23 \mathrm{~A}) \quad 109.5$

$\mathrm{C}(20)-\mathrm{C}(23)-\mathrm{H}(23 \mathrm{~B}) \quad 109.5$

$\mathrm{H}(23 \mathrm{~A})-\mathrm{C}(23)-\mathrm{H}(23 \mathrm{~B}) \quad 109.5$

$\mathrm{C}(20)-\mathrm{C}(23)-\mathrm{H}(23 \mathrm{C}) \quad 109.5$

$\mathrm{H}(23 \mathrm{~A})-\mathrm{C}(23)-\mathrm{H}(23 \mathrm{C}) \quad 109.5$

$\mathrm{H}(23 \mathrm{~B})-\mathrm{C}(23)-\mathrm{H}(23 \mathrm{C}) \quad 109.5$

$\mathrm{C}(29)-\mathrm{C}(24)-\mathrm{C}(25) \quad 117.7(2)$

$\mathrm{C}(29)-\mathrm{C}(24)-\mathrm{S}(1) \quad 122.82(17)$

$\mathrm{C}(25)-\mathrm{C}(24)-\mathrm{S}(1) \quad 119.4(2)$

$\mathrm{C}(26)-\mathrm{C}(25)-\mathrm{C}(24) \quad 121.5(3)$

$\mathrm{C}(26)-\mathrm{C}(25)-\mathrm{H}(25) \quad 119.3$

$\mathrm{C}(24)-\mathrm{C}(25)-\mathrm{H}(25) \quad 119.3$

$\mathrm{C}(25)-\mathrm{C}(26)-\mathrm{C}(27) \quad 120.0(2)$

$\mathrm{C}(25)-\mathrm{C}(26)-\mathrm{H}(26) \quad 120.0$

$\mathrm{C}(27)-\mathrm{C}(26)-\mathrm{H}(26) 120.0$

$\mathrm{C}(28)-\mathrm{C}(27)-\mathrm{C}(26) \quad 118.1(2)$

$\mathrm{C}(28)-\mathrm{C}(27)-\mathrm{H}(27) \quad 121.0$

$\mathrm{C}(26)-\mathrm{C}(27)-\mathrm{H}(27) \quad 121.0$

$\mathrm{C}(27)-\mathrm{C}(28)-\mathrm{C}(29) \quad 121.8(3)$

$\mathrm{C}(27)-\mathrm{C}(28)-\mathrm{H}(28) \quad 119.1$

$\mathrm{C}(29)-\mathrm{C}(28)-\mathrm{H}(28) \quad 119.1$

$\mathrm{C}(24)-\mathrm{C}(29)-\mathrm{C}(28) \quad 120.9(2)$

$\mathrm{C}(24)-\mathrm{C}(29)-\mathrm{H}(29) \quad 119.6$

$\mathrm{C}(28)-\mathrm{C}(29)-\mathrm{H}(29) \quad 119.6$

$\mathrm{C}(31)-\mathrm{C}(30)-\mathrm{C}(35) \quad 119.46(18)$

$\mathrm{C}(31)-\mathrm{C}(30)-\mathrm{S}(2) \quad 122.68(14)$

C(35)-C(30)-S(2) 117.84(15) 
$\mathrm{C}(30)-\mathrm{C}(31)-\mathrm{C}(32) \quad 120.00(19)$

$\mathrm{C}(30)-\mathrm{C}(31)-\mathrm{H}(31) \quad 120.0$

$\mathrm{C}(32)-\mathrm{C}(31)-\mathrm{H}(31) \quad 120.0$

$\mathrm{C}(33)-\mathrm{C}(32)-\mathrm{C}(31)$ 120.1(2)

$\mathrm{C}(33)-\mathrm{C}(32)-\mathrm{H}(32) \quad 119.9$

$\mathrm{C}(31)-\mathrm{C}(32)-\mathrm{H}(32) \quad 119.9$

$\mathrm{C}(34)-\mathrm{C}(33)-\mathrm{C}(32) \quad 120.2(2)$

$\mathrm{C}(34)-\mathrm{C}(33)-\mathrm{H}(33) \quad 119.9$
$\mathrm{C}(32)-\mathrm{C}(33)-\mathrm{H}(33) 119.9$

$\mathrm{C}(33)-\mathrm{C}(34)-\mathrm{C}(35) \quad 119.88(19)$

$\mathrm{C}(33)-\mathrm{C}(34)-\mathrm{H}(34) 120.1$

$\mathrm{C}(35)-\mathrm{C}(34)-\mathrm{H}(34) 120.1$

$\mathrm{C}(34)-\mathrm{C}(35)-\mathrm{C}(30) \quad 120.30(19)$

$\mathrm{C}(34)-\mathrm{C}(35)-\mathrm{H}(35) 119.8$

$\mathrm{C}(30)-\mathrm{C}(35)-\mathrm{H}(35) \quad 119.8$

Symmetry transformations used to generate equivalent atoms:

$\# 1-x+1,-y+1,-z+1$ 
Table S6. Anisotropic displacement parameters $\left(\AA^{2} \times 10^{3}\right)$ for lla.

The anisotropic displacement factor exponent takes the form: $-2 \pi^{2}\left[h^{2} a^{*} U^{11}+\right.$ $\left.\ldots+2 h \mathrm{~h} \mathrm{a}^{*} \mathrm{~b}^{*} \mathrm{U}^{12}\right]$

\begin{tabular}{|c|c|c|c|c|c|c|}
\hline Atom & $\mathrm{U}^{11}$ & $\mathrm{U}^{22}$ & $\mathrm{U}^{33}$ & $\mathrm{U}^{23}$ & $U^{13}$ & $\mathrm{U}^{12}$ \\
\hline $\operatorname{Pd}(1)$ & 11(1) & $13(1)$ & $13(1)$ & $-1(1)$ & $-1(1)$ & $-1(1)$ \\
\hline$S(1)$ & $20(1)$ & $26(1)$ & $23(1)$ & 1(1) & $-7(1)$ & $6(1)$ \\
\hline S(2) & 15(1) & 13(1) & $17(1)$ & $-2(1)$ & $-2(1)$ & $2(1)$ \\
\hline$N(1)$ & $17(1)$ & $24(1)$ & $15(1)$ & $-4(1)$ & $1(1)$ & $-9(1)$ \\
\hline C(2) & 13(1) & $20(1)$ & $14(1)$ & $-1(1)$ & $1(1)$ & $-2(1)$ \\
\hline$N(3)$ & 16(1) & 21(1) & $17(1)$ & $-5(1)$ & $3(1)$ & $-7(1)$ \\
\hline C(4) & $30(1)$ & $30(1)$ & $21(1)$ & $-12(1)$ & $6(1)$ & $-12(1)$ \\
\hline C(5) & $28(1)$ & $34(1)$ & $21(1)$ & $-11(1)$ & $3(1)$ & $-16(1)$ \\
\hline C(6) & $16(1)$ & $25(1)$ & $15(1)$ & $-1(1)$ & 2(1) & $-7(1)$ \\
\hline$C(7)$ & 19(1) & $26(1)$ & $20(1)$ & $1(1)^{\prime}$ & $0(1)$ & $-6(1)$ \\
\hline C(8) & $28(1)$ & $37(1)$ & $20(1)$ & $8(1)$ & 2(1) & $-5(1)$ \\
\hline C(9) & $22(1)$ & $42(1)$ & $25(1)$ & $3(1)$ & $7(1)$ & $-4(1)$ \\
\hline$C(10)$ & $16(1)$ & $36(1)$ & $30(1)$ & $4(1)$ & 1(1) & $-2(1)$ \\
\hline$C(11)$ & $18(1)$ & 29(1) & $20(1)$ & $4(1)$ & $-2(1)$ & $-8(1)$ \\
\hline$C(12)$ & $26(1)$ & $29(1)$ & $32(1)$ & $3(1)$ & $-4(1)$ & $-2(1)$ \\
\hline$C(13)$ & $35(1)$ & $72(2)$ & $37(1)$ & $8(1)$ & $19(1)$ & $0(1)$ \\
\hline$C(14)$ & $24(1)$ & $45(1)$ & $29(1)$ & $14(1)$ & $-5(1)$ & $-8(1)$ \\
\hline$C(15)$ & $16(1)$ & $20(1)$ & $18(1)$ & $-3(1)$ & $5(1)$ & $-4(1)$ \\
\hline$C(16)$ & $19(1)$ & 22(1) & $21(1)$ & $1(1)$ & 4(1) & $0(1)$ \\
\hline$C(17)$ & $23(1)$ & $22(1)$ & $26(1)$ & $2(1)$ & $7(1)$ & $-2(1)$ \\
\hline$C(18)$ & $18(1)$ & $23(1)$ & $26(1)$ & $-4(1)$ & $8(1)$ & $-4(1)$ \\
\hline$C(19)$ & $17(1)$ & $22(1)$ & $27(1)$ & $-3(1)$ & $5(1)$ & $1(1)$ \\
\hline C(20) & $21(1)$ & 15(1) & $21(1)$ & $-3(1)$ & $6(1)$ & $0(1)$ \\
\hline$C(21)$ & $22(1)$ & $45(1)$ & $38(1)$ & $14(1)$ & $6(1)$ & $6(1)$ \\
\hline C(22) & $20(1)$ & $33(1)$ & $45(1)$ & $3(1)$ & $9(1)$ & $-7(1)$ \\
\hline$C(23)$ & $31(1)$ & $16(1)$ & $33(1)$ & $-2(1)$ & $9(1)$ & $2(1)$ \\
\hline$C(24)$ & $20(1)$ & $28(1)$ & $34(1)$ & $-9(1)$ & $-8(1)$ & $4(1)$ \\
\hline$C(25)$ & $38(1)$ & $34(1)$ & $49(2)$ & $-6(1)$ & $-10(1)$ & $11(1)$ \\
\hline$C(26)$ & $54(2)$ & 49(2) & $60(2)$ & $-28(2)$ & $-15(1)$ & $28(1)$ \\
\hline C(27) & $25(1)$ & 72(2) & $59(2)$ & $-40(2)$ & $3(1)$ & $7(1)$ \\
\hline C(28) & $34(1)$ & $48(2)$ & $42(1)$ & $-13(1)$ & $3(1)$ & $-4(1)$ \\
\hline C(29) & $27(1)$ & $33(1)$ & $35(1)$ & $-7(1)$ & $-1(1)$ & $2(1)$ \\
\hline$C(30)$ & $20(1)$ & 12(1) & $20(1)$ & $-1(1)$ & $-4(1)$ & $-2(1)$ \\
\hline$C(31)$ & $30(1)$ & $18(1)$ & $25(1)$ & $1(1)$ & $4(1)$ & $1(1)$ \\
\hline$C(32)$ & $36(1)$ & $22(1)$ & $29(1)$ & $1(1)$ & $7(1)$ & $-4(1)$ \\
\hline C(33) & $35(1)$ & $23(1)$ & $29(1)$ & $7(1)$ & $-5(1)$ & $-10(1)$ \\
\hline$C(34)$ & $29(1)$ & $16(1)$ & $41(1)$ & $9(1)$ & $-9(1)$ & $-2(1)$ \\
\hline C(35) & $20(1)$ & $16(1)$ & $33(1)$ & $2(1)$ & $-3(1)$ & $0(1)$ \\
\hline
\end{tabular}


Table S7. Hydrogen coordinates $\left(x 1^{4}\right)$ and isotropic displacement parameters $\left(\AA^{2} \times 10^{3}\right)$ for Ila.

\begin{tabular}{|c|c|c|c|c|}
\hline Atom & $x$ & $y$ & $z$ & U(iso) \\
\hline & & 3953 & 1911 & $1988 \quad 32$ \\
\hline$H(5)$ & 2651 & 1426 & 2702 & 33 \\
\hline $\mathrm{H}(8)$ & 1900 & 1593 & 5786 & 34 \\
\hline $\mathrm{H}(10)$ & 508 & 3773 & 4368 & 33 \\
\hline$H(12 A)$ & 3251 & 862 & 5419 & 44 \\
\hline$H(12 B)$ & 3758 & 1577 & 4766 & 44 \\
\hline $\mathrm{H}(12 \mathrm{C})$ & 3156 & 498 & 4499 & 44 \\
\hline$H(13 A)$ & 301 & 2013 & 5983 & 72 \\
\hline$H(13 B)$ & -63 & 3194 & 5576 & 72 \\
\hline$H(13 C)$ & 673 & 3266 & 6262 & 72 \\
\hline$H(14 A)$ & 1001 & 4473 & 3147 & 49 \\
\hline$H(14 B)$ & 1396 & 3371 & 2696 & 49 \\
\hline$H(14 C)$ & 2003 & 4423 & 3003 & 49 \\
\hline $\mathrm{H}(17)$ & 5723 & 5900 & 1726 & 28 \\
\hline $\mathrm{H}(19)$ & 7031 & 3298 & 2858 & 26 \\
\hline$H(21 A)$ & 3681 & 4677 & 1905 & 52 \\
\hline$H(21 B)$ & 4196 & 5699 & 1477 & 52 \\
\hline$H(21 C)$ & 3953 & 5840 & 2389 & 52 \\
\hline$H(22 A)$ & 7755 & 4690 & 1959 & 49 \\
\hline$H(22 B)$ & 7539 & 5757 & 2540 & 49 \\
\hline$H(22 C)$ & 7237 & 5807 & 1619 & 49 \\
\hline$H(23 A)$ & 6292 & 1875 & 3664 & 40 \\
\hline$H(23 B)$ & 5754 & 1303 & 2933 & 40 \\
\hline$H(23 C)$ & 5272 & 1866 & 3664 & 40 \\
\hline$H(25)$ & 2234 & 8048 & 4270 & 48 \\
\hline$H(26)$ & 1364 & 8574 & 5283 & 66 \\
\hline $\mathrm{H}(27)$ & 1025 & 7155 & 6294 & 62 \\
\hline$H(28)$ & 1621 & 5309 & 6238 & 49 \\
\hline $\mathrm{H}(29)$ & 2529 & 4794 & 5232 & 38 \\
\hline$H(31)$ & 4033 & 3850 & 6041 & 29 \\
\hline$H(32)$ & 3632 & 2601 & 7069 & 35 \\
\hline $\mathrm{H}(33)$ & 4331 & 803 & 7277 & 35 \\
\hline $\mathrm{H}(34)$ & 5423 & 242 & 6457 & 35 \\
\hline$H(35)$ & 5831 & 1486 & 5429 & 28 \\
\hline
\end{tabular}


Table S8. Torsion angles $\left[^{\circ}\right]$ for lla.

\begin{tabular}{|c|c|c|c|}
\hline$C(5)-N(1)-C(2)-N(3)$ & $0.0(2)$ & $N(3)-C(15)-C(16)-C(17)$ & $-179.88(17)$ \\
\hline$C(6)-N(1)-C(2)-N(3)-$ & $179.10(17)$ & $C(20)-C(15)-C(16)-C(21)$ & $177.97(19)$ \\
\hline$C(5)-N(1)-C(2)-P d(1)$ & $177.11(16)$ & $N(3)-C(15)-C(16)-C(21)$ & $0.3(3)$ \\
\hline $\mathrm{C}(6)-\mathrm{N}(1)-\mathrm{C}(2)-\mathrm{Pd}(1)$ & $-2.0(3)$ & $C(15)-C(16)-C(17)-C(18)$ & $-0.7(3)$ \\
\hline$N(1)-C(2)-N(3)-C(4)$ & $0.1(2)$ & $C(21)-C(16)-C(17)-C(18)$ & $179.1(2)$ \\
\hline$P d(1)-C(2)-N(3)-C(4)$ & $-177.33(14)$ & $C(16)-C(17)-C(18)-C(19)$ & $2.5(3)$ \\
\hline$N(1)-C(2)-N(3)-C(15)$ & $179.30(17)$ & $C(16)-C(17)-C(18)-C(22)$ & $-177.0(2)$ \\
\hline $\operatorname{Pd}(1)-C(2)-N(3)-C(15)$ & $1.8(3)$ & $C(17)-C(18)-C(19)-C(20)$ & $-1.5(3)$ \\
\hline$C(2)-N(3)-C(4)-C(5)$ & $-0.2(3)$ & $C(22)-C(18)-C(19)-C(20)$ & $177.98(19)$ \\
\hline$C(15)-N(3)-C(4)-C(5)$ & $-179.39(19)$ & $C(16)-C(15)-C(20)-C(19)$ & $3.1(3)$ \\
\hline$N(3)-C(4)-C(5)-N(1)$ & $0.2(3)$ & $N(3)-C(15)-C(20)-C(19)$ & $-179.15(16)$ \\
\hline$C(2)-N(1)-C(5)-C(4)$ & $-0.1(3)$ & $C(16)-C(15)-C(20)-C(23)$ & $-174.06(18)$ \\
\hline$C(6)-N(1)-C(5)-C(4)$ & $178.98(19)$ & $N(3)-C(15)-C(20)-C(23)$ & $3.7(3)$ \\
\hline$C(2)-N(1)-C(6)-C(7)$ & $-86.7(2)$ & $C(18)-C(19)-C(20)-C(15)$ & $-1.2(3)$ \\
\hline $\mathrm{C}(5)-\mathrm{N}(1)-\mathrm{C}(6)-\mathrm{C}(7)$ & $94.3(2)$ & $C(18)-C(19)-C(20)-C(23)$ & $176.00(18)$ \\
\hline$C(2)-N(1)-C(6)-C(11)$ & $96.9(2)$ & $P d(1)-S(1)-C(24)-C(29)$ & $-37.17(19)$ \\
\hline$C(5)-N(1)-C(6)-C(11)$ & $-82.0(3)$ & $\mathrm{Pd}(1)-\mathrm{S}(1)-\mathrm{C}(24)-\mathrm{C}(25)$ & $145.97(17)$ \\
\hline$C(11)-C(6)-C(7)-C(8)$ & $-7.0(3)$ & $C(29)-C(24)-C(25)-C(26)$ & $-0.5(4)$ \\
\hline$N(1)-C(6)-C(7)-C(8)$ & $176.73(17)$ & $S(1)-C(24)-C(25)-C(26)$ & $176.5(2)$ \\
\hline$C(11)-C(6)-C(7)-C(12)$ & 169.61(19) & $C(24)-C(25)-C(26)-C(27)$ & $-0.7(4)$ \\
\hline$N(1)-C(6)-C(7)-C(12)$ & $-6.6(3)$ & $C(25)-C(26)-C(27)-C(28)$ & $1.1(4)^{\prime}$ \\
\hline$C(6)-C(7)-C(8)-C(9)$ & $2.3(3)$ & $C(26)-C(27)-C(28)-C(29)$ & $-0.2(4)$ \\
\hline$C(12)-C(7)-C(8)-C(9)$ & $-174.4(2)$ & $C(25)-C(24)-C(29)-C(28)$ & $1.4(3)$ \\
\hline$C(7)-C(8)-C(9)-C(10)$ & $2.9(3)$ & $S(1)-C(24)-C(29)-C(28)$ & $-175.50(18)$ \\
\hline$C(7)-C(8)-C(9)-C(13)$ & $-177.3(2)$ & $C(27)-C(28)-C(29)-C(24)$ & $-1.1(4)$ \\
\hline $\mathrm{C}(8)-\mathrm{C}(9)-\mathrm{C}(10)-\mathrm{C}(11)$ & $-3.6(3)$ & $P d(1)-S(2)-C(30)-C(31)$ & $-35.21(17)$ \\
\hline$C(13)-C(9)-C(10)-C(11)$ & 176.6(2) & $\mathrm{Pd}(1) \# 1-\mathrm{S}(2)-\mathrm{C}(30)-\mathrm{C}(31)$ & $63.90(16)$ \\
\hline$C(9)-C(10)-C(11)-C(6)$ & $-0.9(3)$ & $P d(1)-S(2)-C(30)-C(35)$ & $146.80(13)$ \\
\hline$C(9)-C(10)-C(11)-C(14)$ & $176.9(2)$ & $\mathrm{Pd}(1) \# 1-\mathrm{S}(2)-\mathrm{C}(30)-\mathrm{C}(35)$ & $-114.09(14)$ \\
\hline$C(7)-C(6)-C(11)-C(10)$ & $6.4(3)$ & $C(35)-C(30)-C(31)-C(32)$ & $0.1(3)$ \\
\hline$N(1)-C(6)-C(11)-C(10)$ & $-177.40(18)$ & $S(2)-C(30)-C(31)-C(32)$ & $-177.83(16)$ \\
\hline$C(7)-C(6)-C(11)-C(14)$ & $-171.39(19)$ & $C(30)-C(31)-C(32)-C(33)$ & $0.0(3)$ \\
\hline$N(1)-C(6)-C(11)-C(14)$ & $4.8(3)$ & $C(31)-C(32)-C(33)-C(34)$ & $-0.2(3)$ \\
\hline$C(2)-N(3)-C(15)-C(20)$ & $96.2(2)$ & $C(32)-C(33)-C(34)-C(35)$ & $0.3(3)$ \\
\hline$C(4)-N(3)-C(15)-C(20)$ & $-84.8(2)$ & $C(33)-C(34)-C(35)-C(30)$ & $-0.2(3)$ \\
\hline$C(2)-N(3)-C(15)-C(16)$ & $-86.0(2)$ & $C(31)-C(30)-C(35)-C(34)$ & $0.0(3)$ \\
\hline$C(4)-N(3)-C(15)-C(16)$ & $93.0(2)$ & $S(2)-C(30)-C(35)-C(34)$ & $178.02(15)$ \\
\hline$C(20)-C(15)-C(16)-C(17)$ & $-2.2(3)$ & & \\
\hline
\end{tabular}

Symmetry transformations used to generate equivalent atoms:

$\# 1-x+1,-y+1,-z+1$ 


\section{S10. References}

(1) (a) Krishnan, R.; Binkley, J. S.; Seeger, R.; Pople, J. A. J. Chem. Phys. 1980, 72, 650-654. (b) Mclean, A. D.; Chandler, G. S. J. Chem. Phys. 1980, 72, 5639-5648.

(2) (a) Schwerdtfeger, P.; Dolg, M.; Schwarz, W. H. E.; Bowmaker, G. A.; Boyd, P. D. W. J. Chem. Phys. 1989, 91, 1762-1774. (b) Andrae, D.; Haußermann, U.; Dolg, M.; Stoll, H.; Preuß, H. Theor. Chim. Acta 1990, 77, 123-141. (c) Bergner, A.; Dolg, M.; Kuchle, W.; Stoll, H.; Preuß, H. Mol. Phys. 1993, 80, 1431-1441.

(3) (a) Perdew, J.P., Burke, K., Ernzerhof, M. Phys. Rev. Lett. 1996, 77, 3865-3868. (b) Adamo, C.; Barone, V. J. Chem. Phys. 1999, 110, 6158-6170.

(4) Frisch, M. J.; Trucks, G. W.; Schlegel, H. B.; Scuseria, G. E.; Robb, M. A.; Cheeseman, J. R.; Scalmani, G.; Barone, V.; Mennucci, B.; Petersson, G. A.; Nakatsuji, H.; Caricato, M.; Li, X.; Hratchian, H. P.; Izmaylov, A. F.; Bloino, J.; Zheng, G.; Sonnenberg, J. L.; Hada, M.; Ehara, M.; Toyota, K.; Fukuda, R.; Hasegawa, J.; Ishida, M.; Nakajima, T.; Honda, Y.; Kitao, O.; Nakai, H.; Vreven, T.; Montgomery, J. A., Jr.; Peralta, J. E.; Ogliaro, F.; Bearpark, M.; Heyd, J. J.; Brothers, E.; Kudin, K. N.; Staroverov, V. N.; Kobayashi, R.; Normand, J.; Raghavachari, K.; Rendell, A.; Burant, J. C.; lyengar, S. S.; Tomasi, J.; Cossi, M.; Rega, N.; Millam, N. J.; Klene, M.; Knox, J. E.; Cross, J. B.; Bakken, V.; Adamo, C.; Jaramillo, J.; Gomperts, R.; Stratmann, R. E.; Yazyev, O.; Austin, A. J.; Cammi, R.; Pomelli, C.; Ochterski, J. W.; Martin, R. L.; Morokuma, K.; Zakrzewski, V. G.; Voth, G. A.; Salvador, P.; Dannenberg, J. J.; Dapprich, S.; Daniels, A. D.; Farkas, O.; Foresman, J. B.; Ortiz, J. V.; Cioslowski, J.; Fox, D. J. Gaussian 09, Revision D.01; Gaussian, Inc.: Wallingford, CT, 2009.

(5) (a) Hratchian, H.P.; Schlegel, H.B. J. Chem. Phys. 2004, 120, 9918-9924. (b) Hratchian, H.P.; Schlegel, H.B. J. Chem. Theory Comput. 2005, 1, 61-69.

(6) Pedersen, D.; Rosenbohm, C. Synthesis 2004, 2431-2434.

(7) Weiss, C. J.; Wobser, S. D.; Marks, T. J. J. Am. Chem. Soc. 2009, 131, 20622063.

(8) Weiss, C. J.; Marks, T. J. J. Am. Chem. Soc. 2010, 132, 10533-10546.

(9) Ananikov, V. P.; Orlov, N. V; Beletskaya, I. P.; Khrustalev, V. N.; Antipin, M. Y.; Timofeeva, T. V. J. Am. Chem. Soc. 2007, 129, 7252-7253.

(10) Kuniyasu, H.; Ogawa, A.; Sato, K.; Ryu, I.; Kambe, N.; Sonoda, N. J. Am. Chem. Soc. 1992, 114, 5902-5903.

(11) Ananikov, V. P.; Orlov, N. V.; Beletskaya, I. P. Organometallics 2007, 26, 740750.

(12) Satoh, T.; Takahashi, Y.; Shirai, Y.; Yamada, Y. Chem. Pharm. Bull. 2006, 54, 1734-1738.

(13) Marion, N.; de Frémont, P.; Puijk, I. M.; Ecarnot, E. C.; Amoroso, D.; Bell, A.; Nolan, S. P. Adv. Synth. Catal. 2007, 349, 2380-2384.

(14) Hintermann, L. Beilstein J. Org. Chem. 2007, 3, №22.

(15) Arduengo, A. J.; Krafczyk, R.; Schmutzler, R.; Craig, H. A.; Goerlich, J. R.; Marshall, W. J.; Unverzagt, M. Tetrahedron 1999, 55, 14523-14534.

(16) Sheldrick G. M., SADABS, v. 2.03, Bruker/Siemens Area Detector Absorption Correction Program, Bruker AXS, Madison, Wisconsin, 2003.

(17) Sheldrick G. M., Acta Cryst., 2008, A64, 112-122.

Acknowledgements. The authors thank Dr. Mikhail Nechaev for providing samples of expanded-ring $\mathrm{N}$-heterocyclic carbine complexes. 
S11. NMR Spectra 
Hept-1-en-2-yl(pentyl)sulfane (3a)

${ }^{1} \mathrm{H}$ NMR $\left(500 \mathrm{MHz}, \mathrm{C}_{6} \mathrm{D}_{6}\right)$
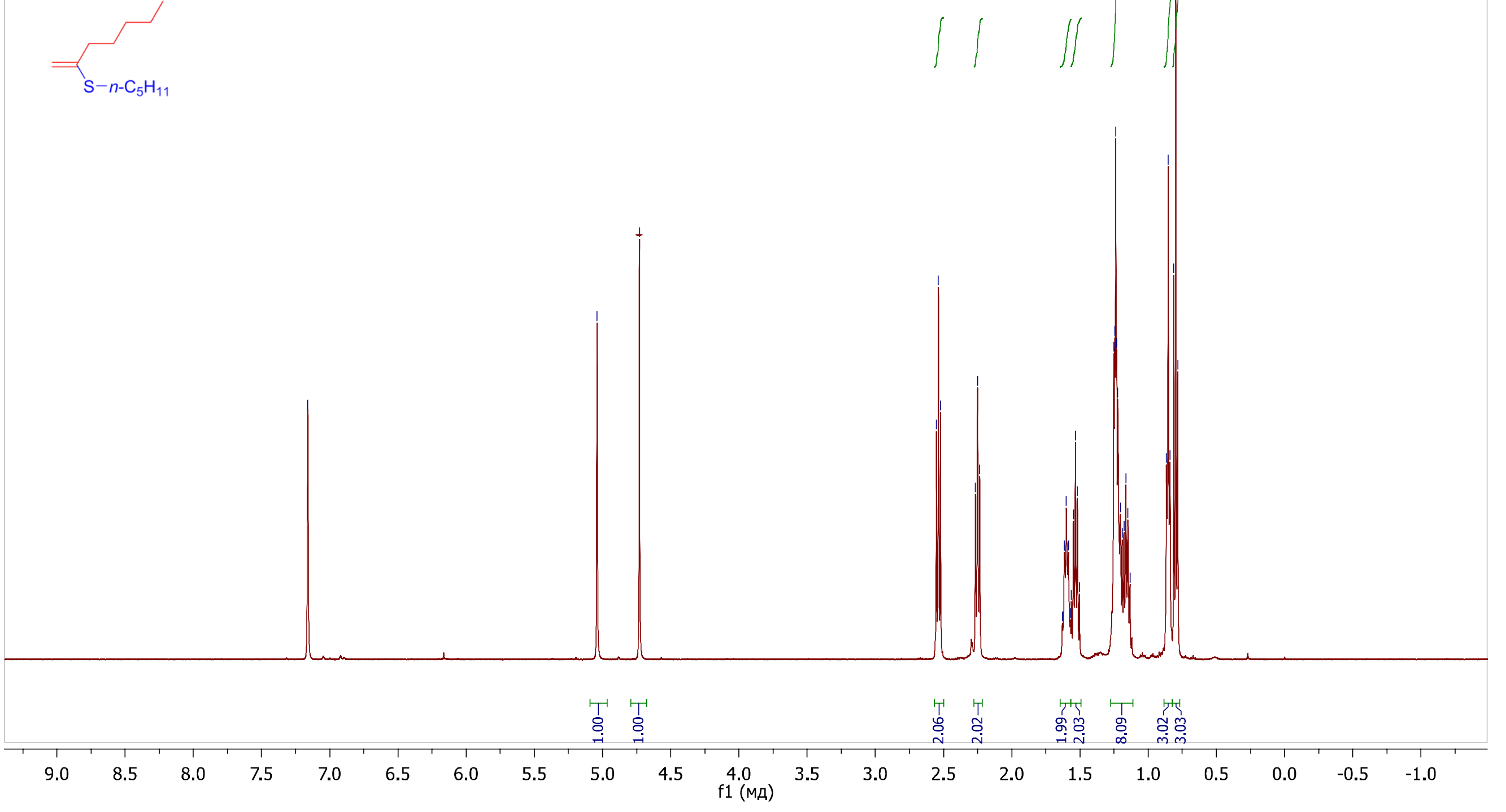


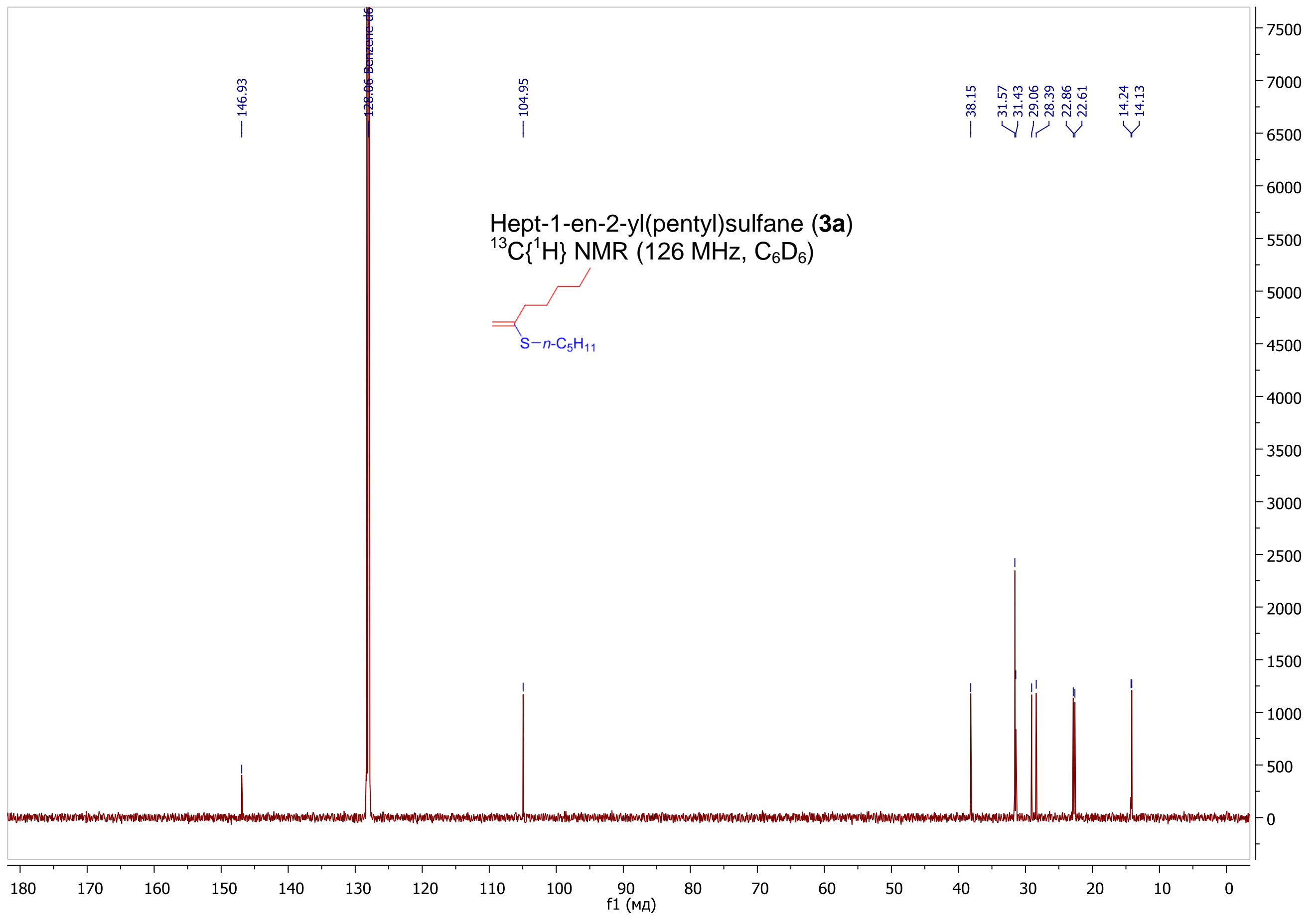




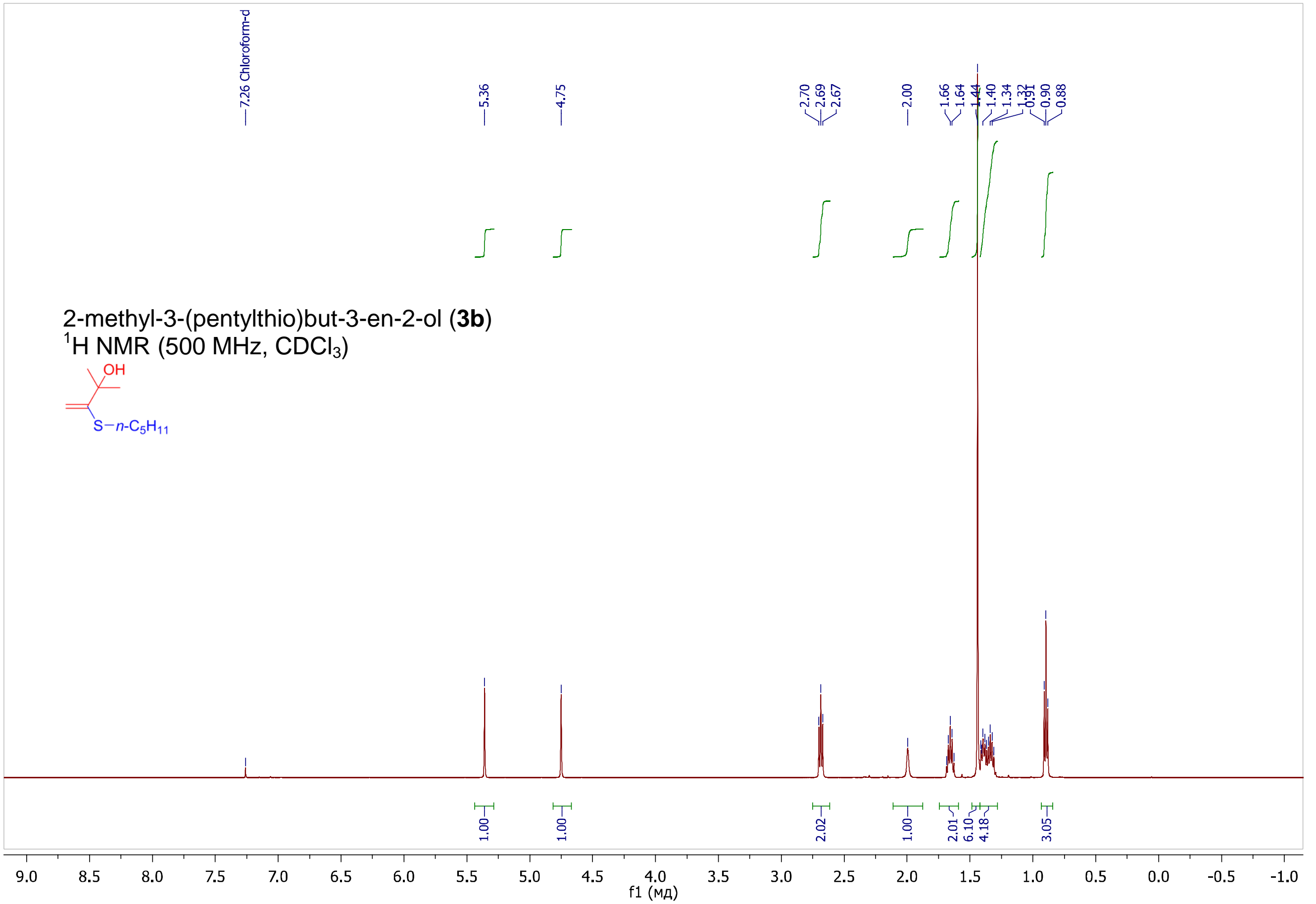


2-methyl-3-(pentylthio)but-3-en-2-ol (3b)

${ }^{13} \mathrm{C}\left\{{ }^{1} \mathrm{H}\right\} \mathrm{NMR}\left(126 \mathrm{MHz}, \mathrm{CDCl}_{3}\right)$

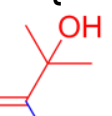

$\mathrm{S}-n-\mathrm{C}_{5} \mathrm{H}_{11}$ 


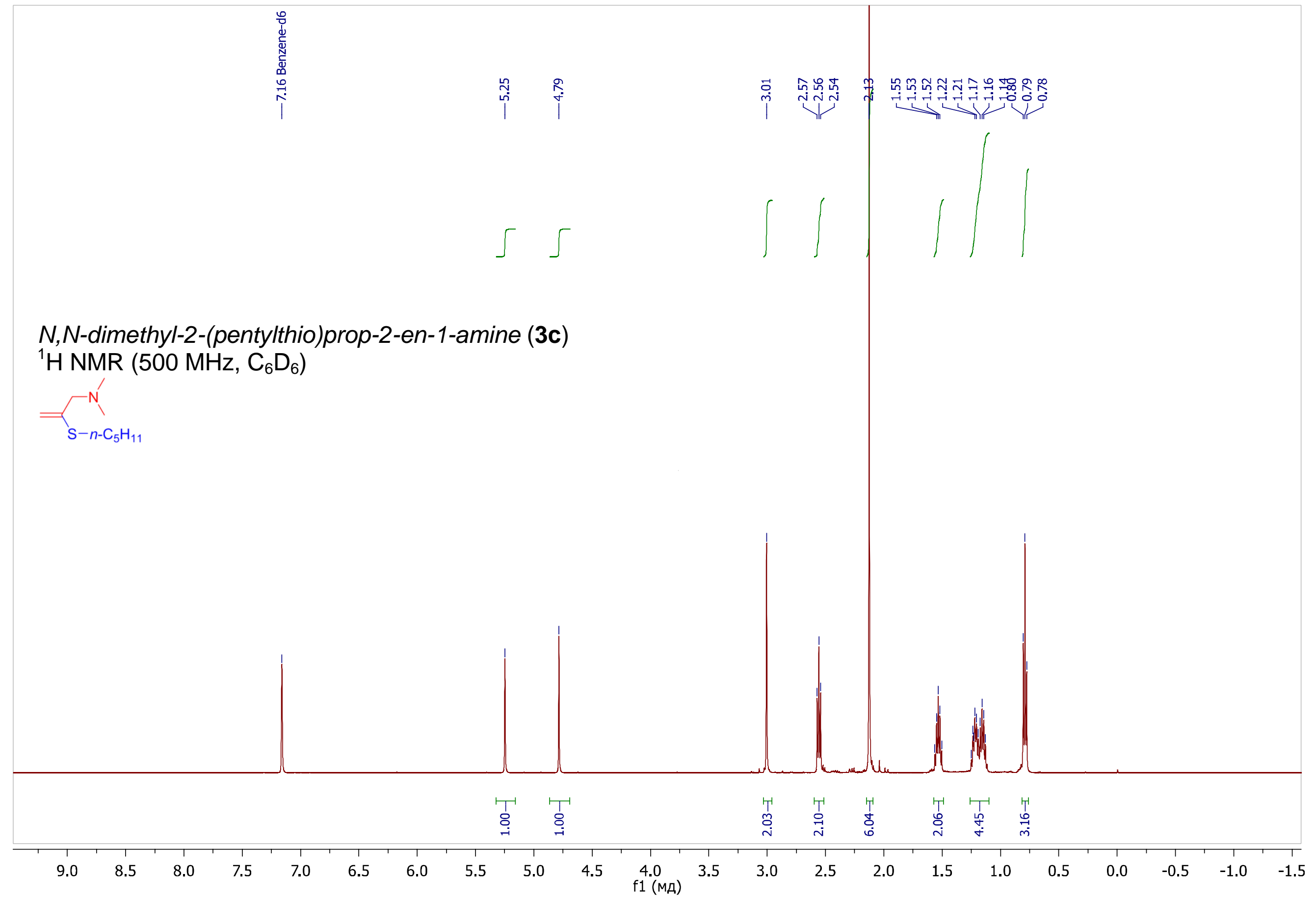




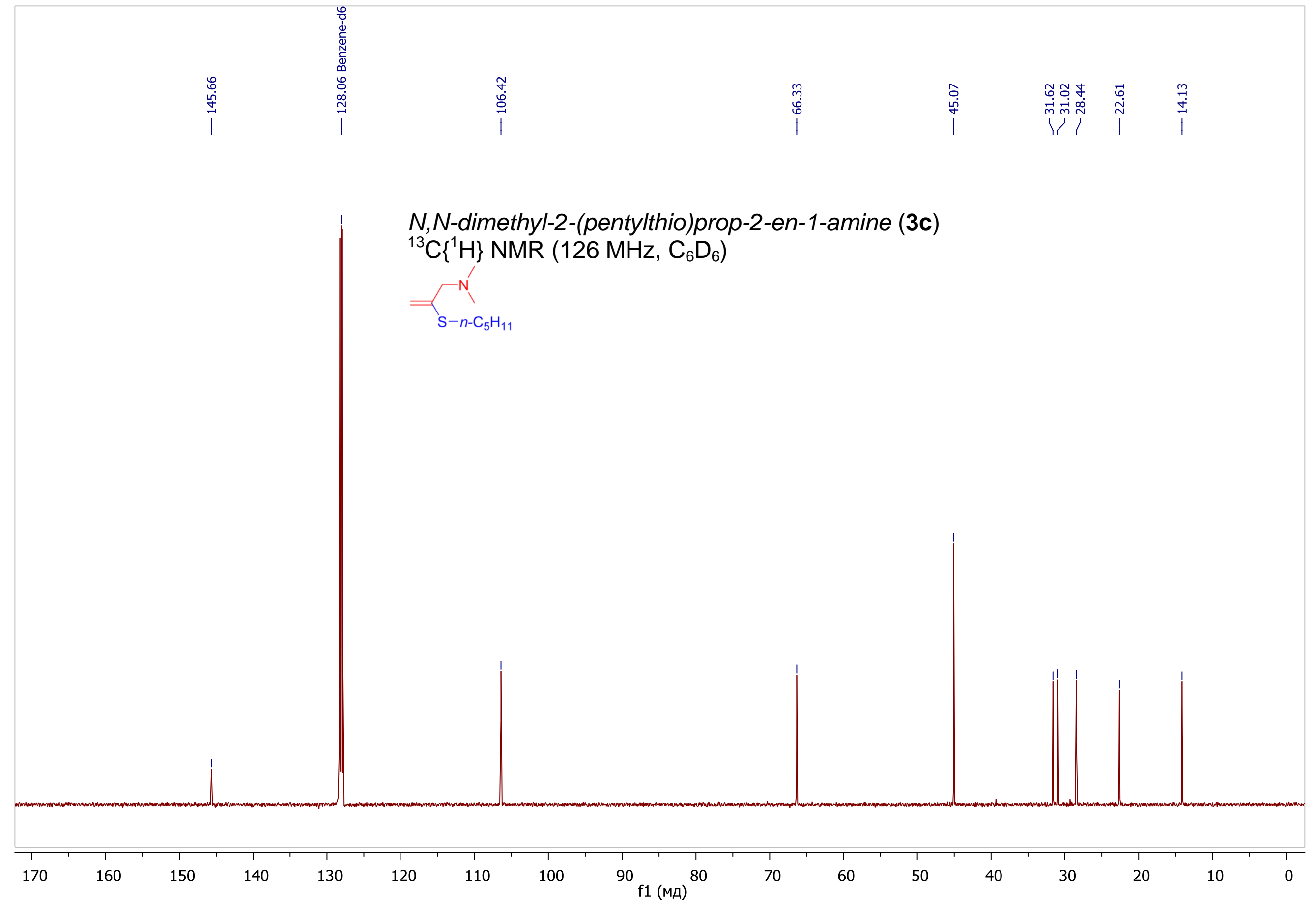




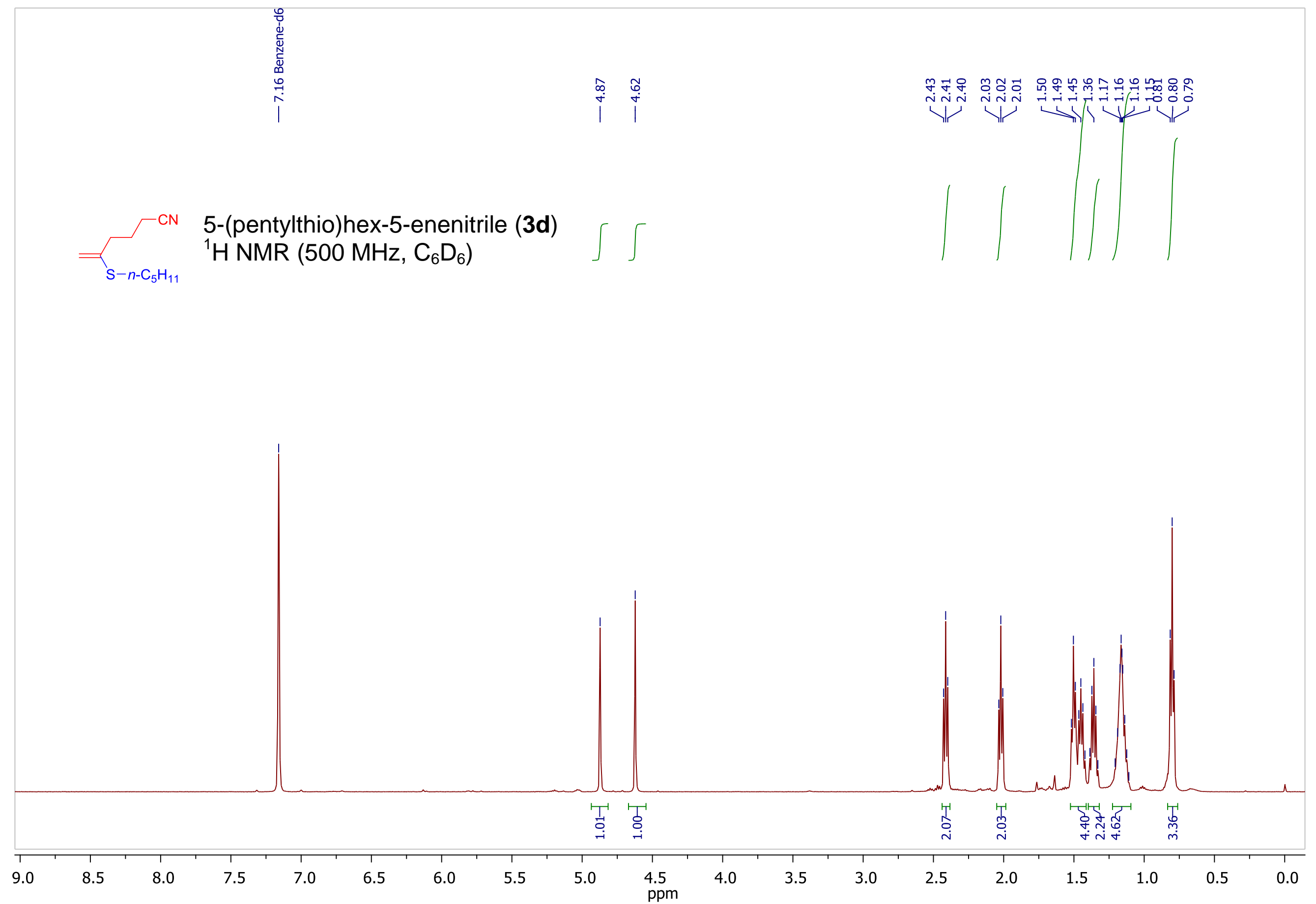




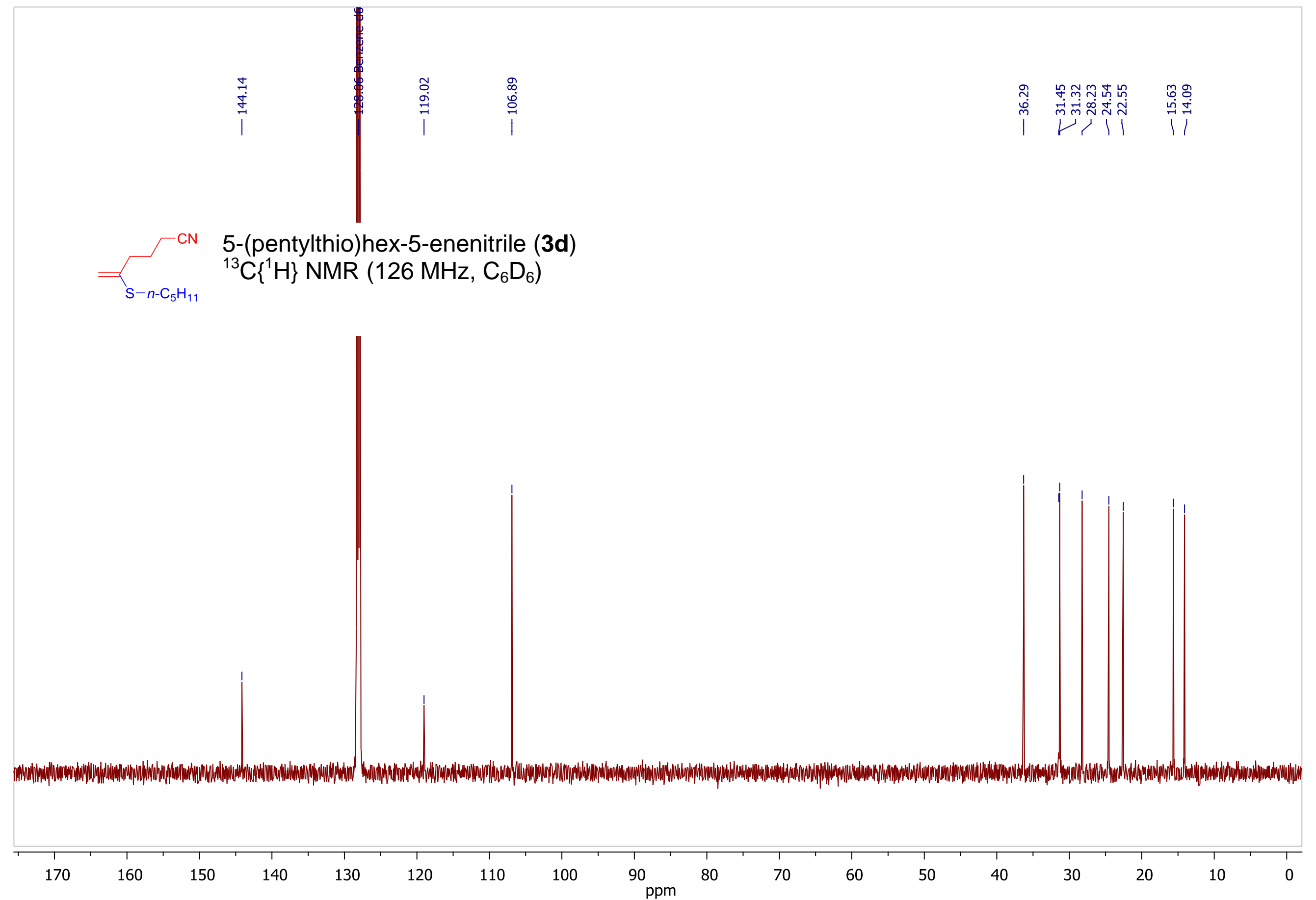




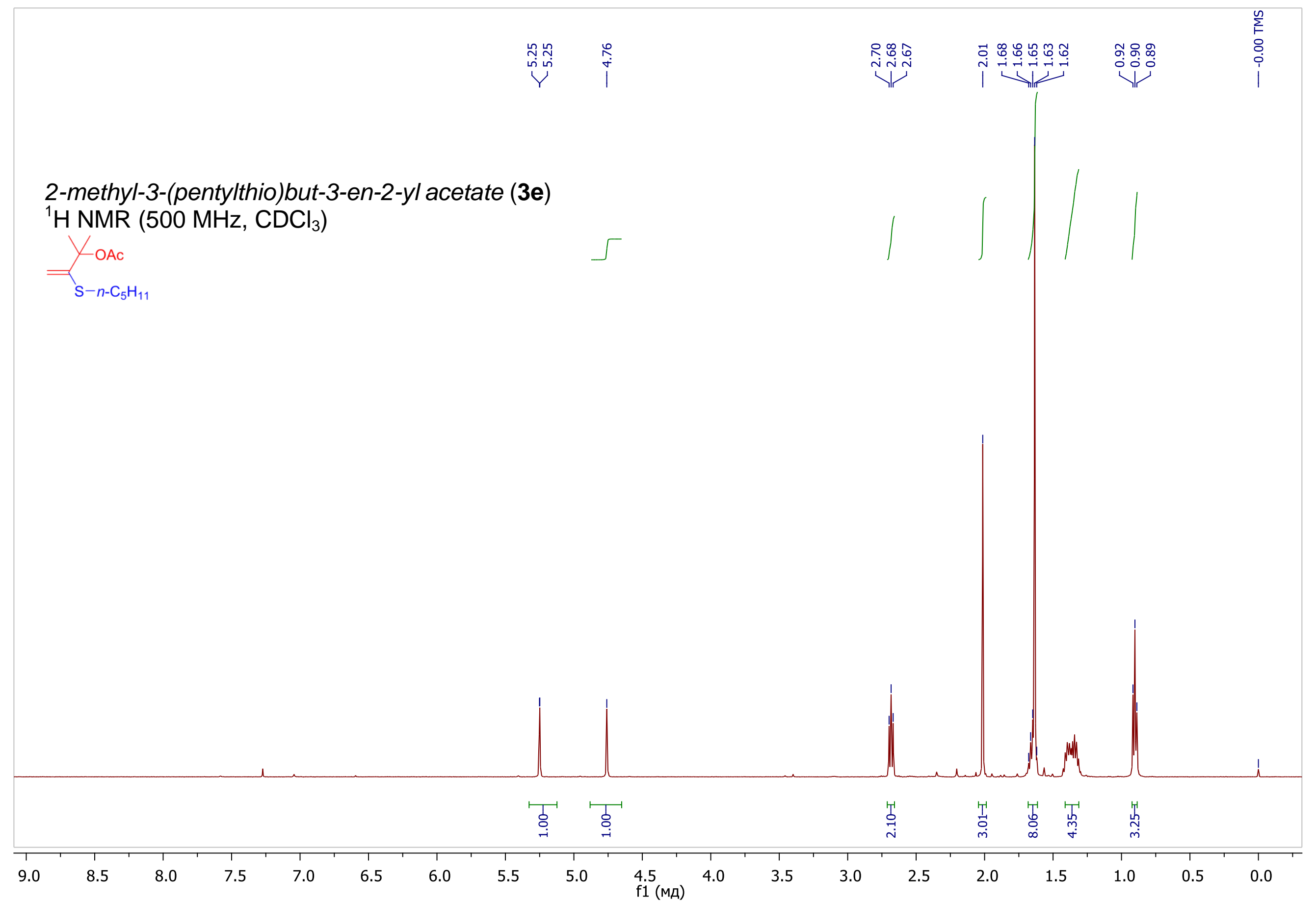


2-methyl-3-(pentylthio)but-3-en-2-yl acetate (3e) ${ }^{13} \mathrm{C}\left\{{ }^{1} \mathrm{H}\right\} \operatorname{NMR}\left(126 \mathrm{MHz}, \mathrm{CDCl}_{3}\right)$

$=\sum_{S-n-\mathrm{C}_{5} \mathrm{H}_{11}}^{\mathrm{OAc}}$

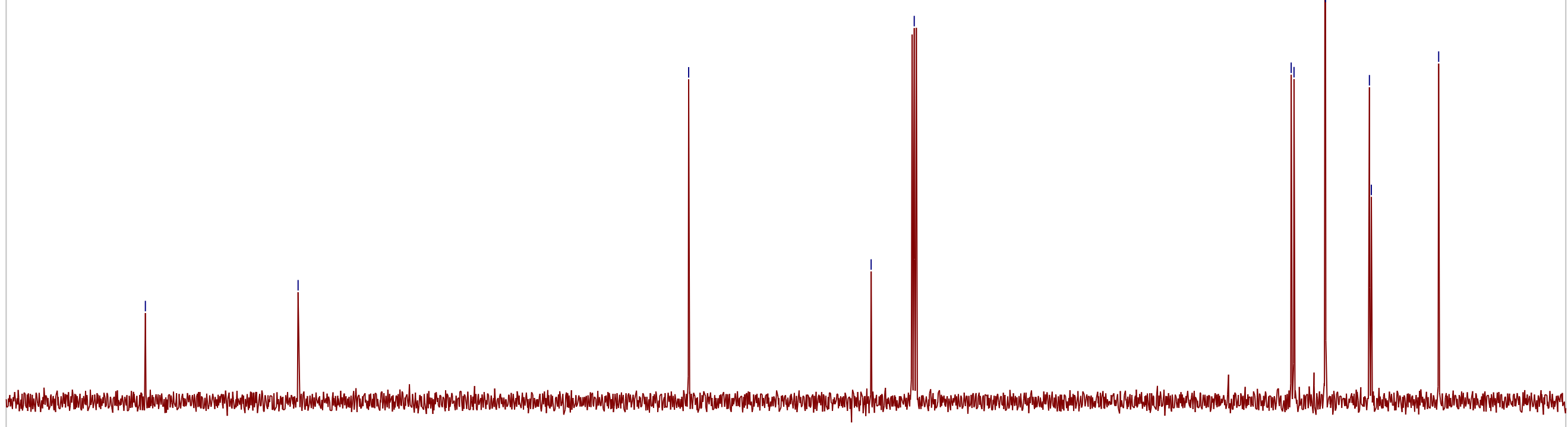

\begin{tabular}{|c|c|c|c|c|c|c|c|c|c|c|c|c|c|c|c|c|c|c|}
\hline 1 & 1 & 1 & 1 & 1 & 1 & 1 & $T$ & 1 & 1 & 1 & 1 & 1 & 1 & $T$ & 1 & 1 & 1 & $T$ \\
\hline 180 & 170 & 160 & 150 & 140 & 130 & 120 & 110 & 100 & $\begin{array}{r}90 \\
\text { f1 (мд) }\end{array}$ & 80 & 70 & 60 & 50 & 40 & 30 & 20 & 10 & 0 \\
\hline
\end{tabular}




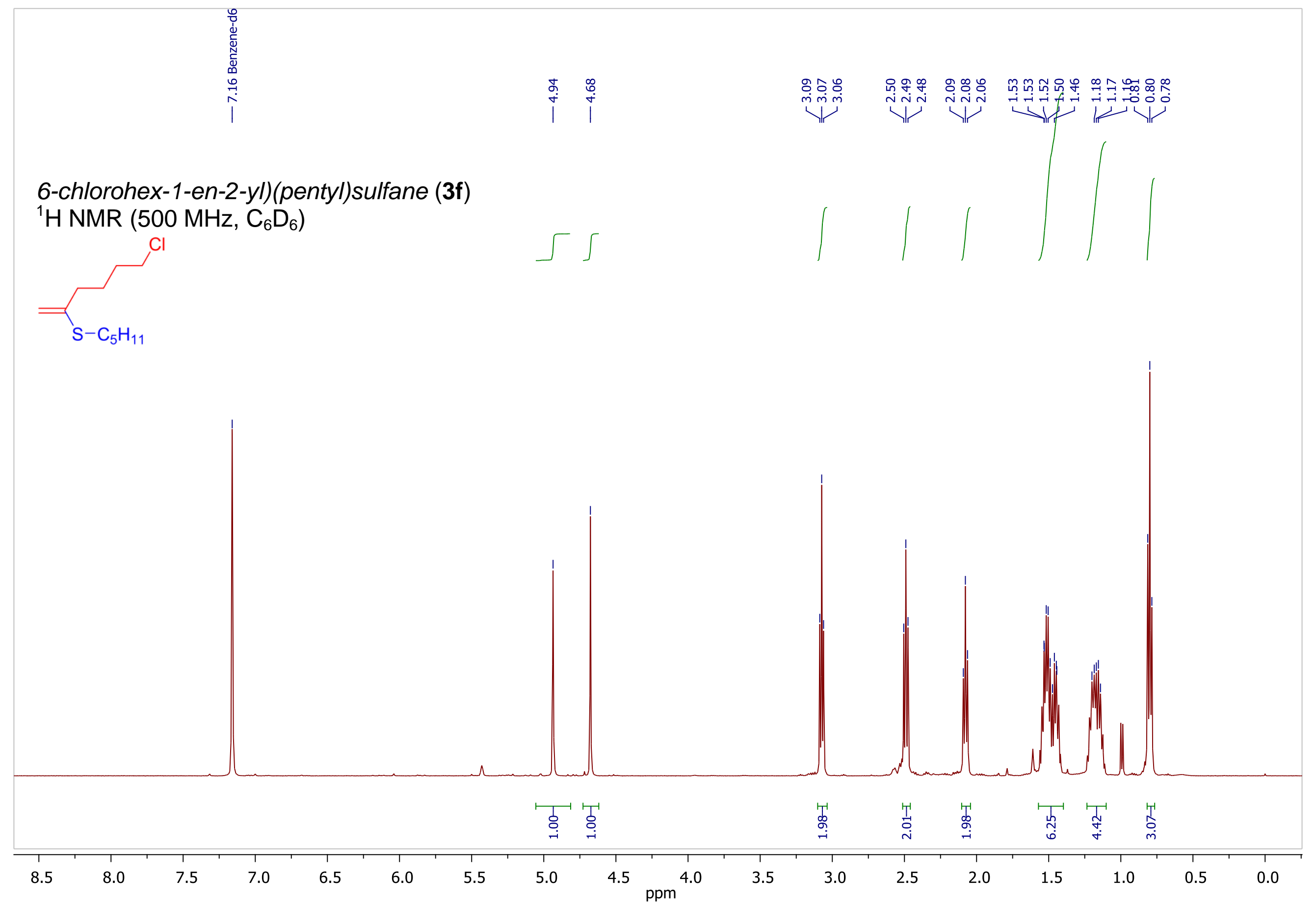




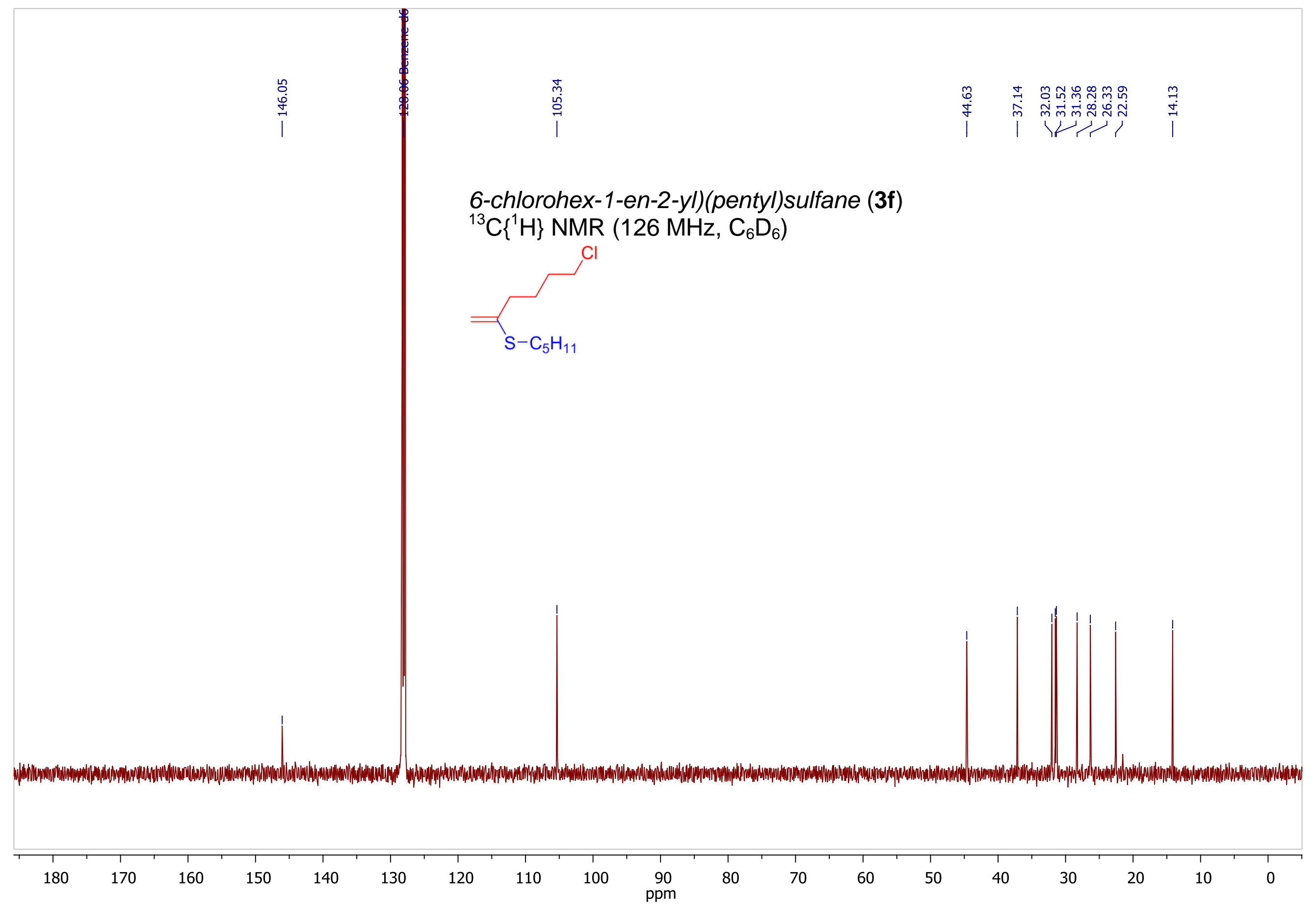




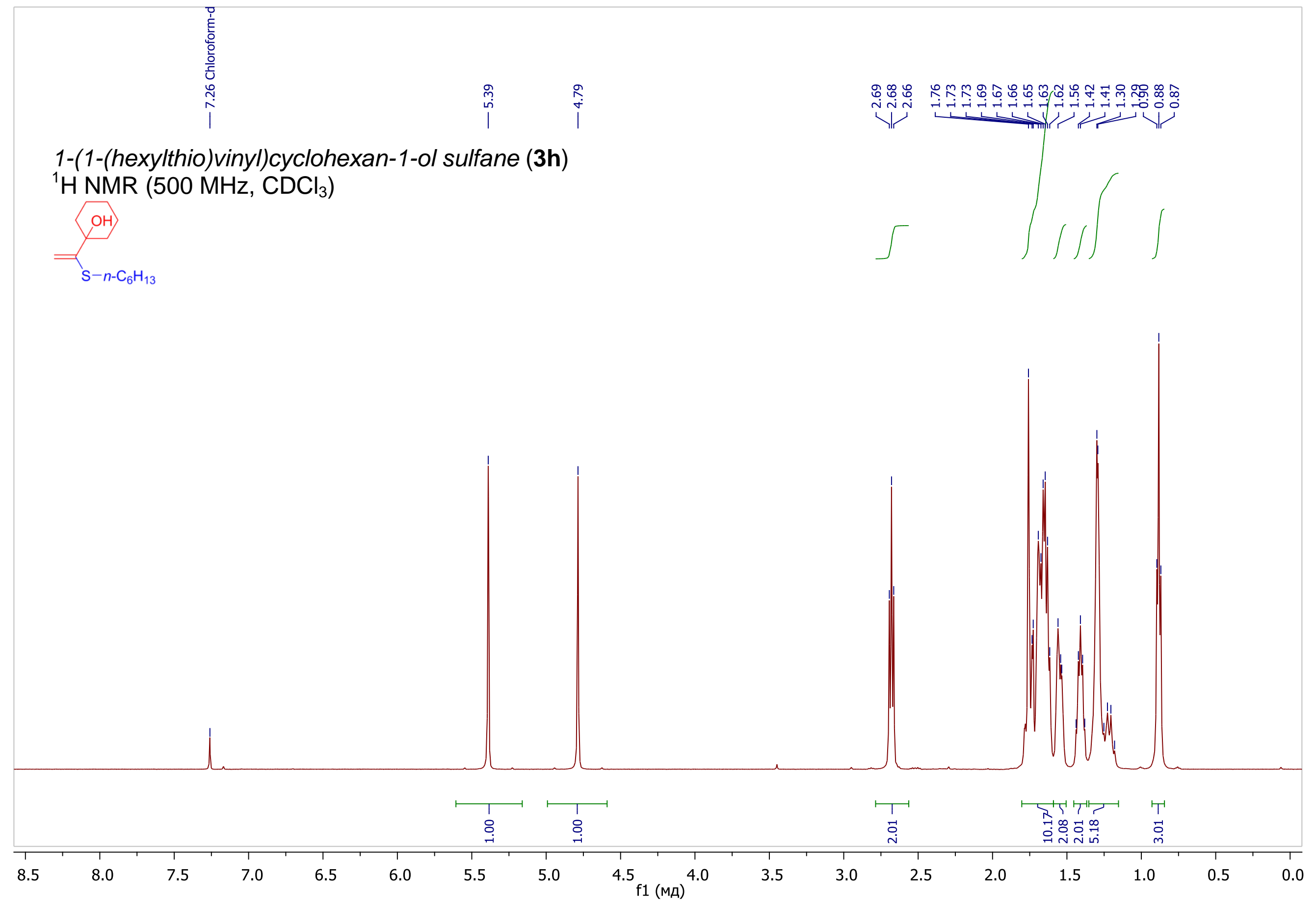




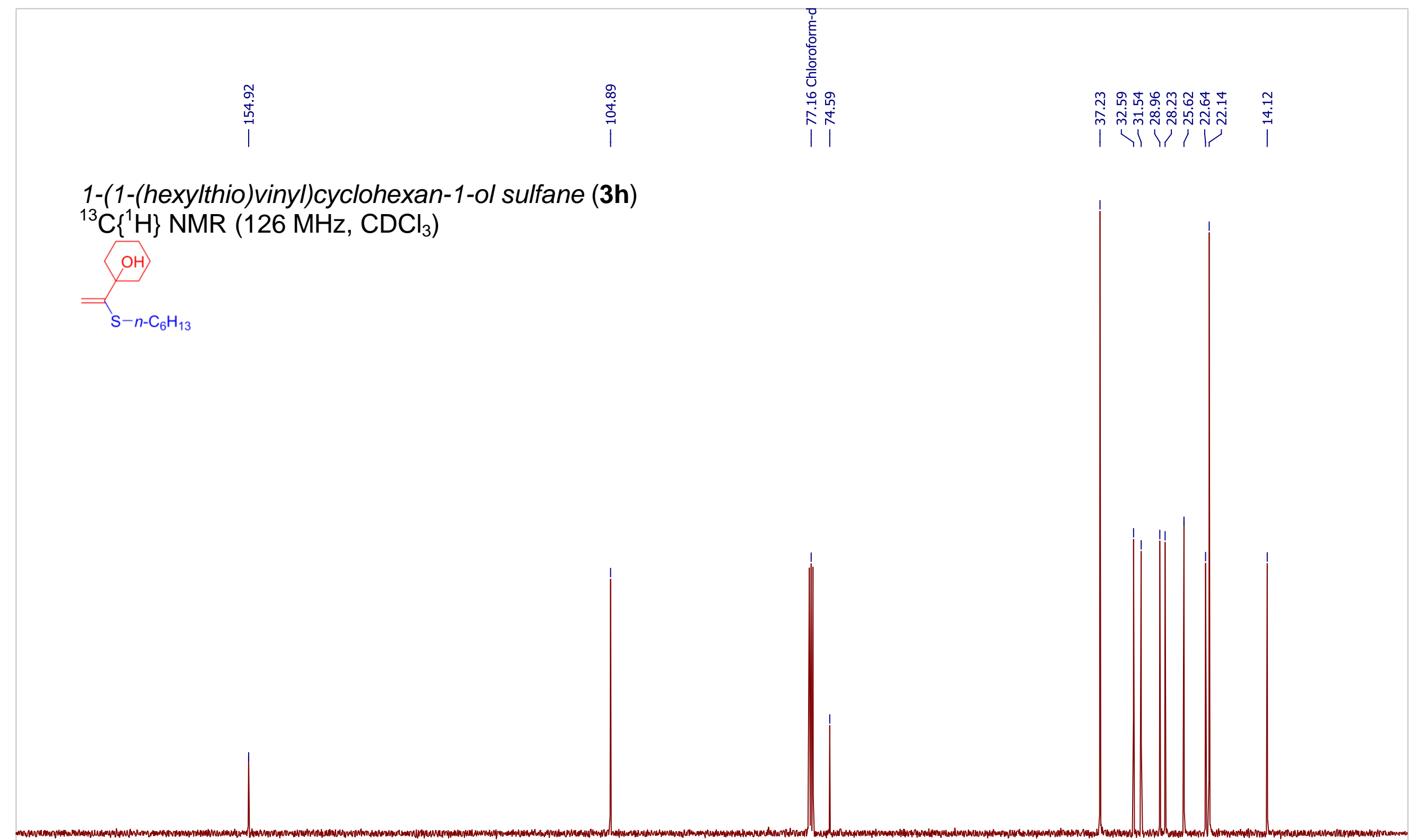

\begin{tabular}{|c|c|c|c|c|c|c|c|c|c|c|c|c|c|c|c|c|c|c|}
\hline 1 & 1 & 1 & 1 & 1 & $T$ & $T$ & 1 & $T$ & 1 & $T$ & $T$ & $T$ & $T$ & $T$ & $T$ & $T$ & $T$ & $T$ \\
\hline 180 & 170 & 160 & 150 & 140 & 130 & 120 & 110 & 100 & $\begin{array}{c}90 \\
\mathrm{f} 1 \stackrel{(\mathrm{MA})}{ }\end{array}$ & 80 & 70 & 60 & 50 & 40 & 30 & 20 & 10 & 0 \\
\hline
\end{tabular}




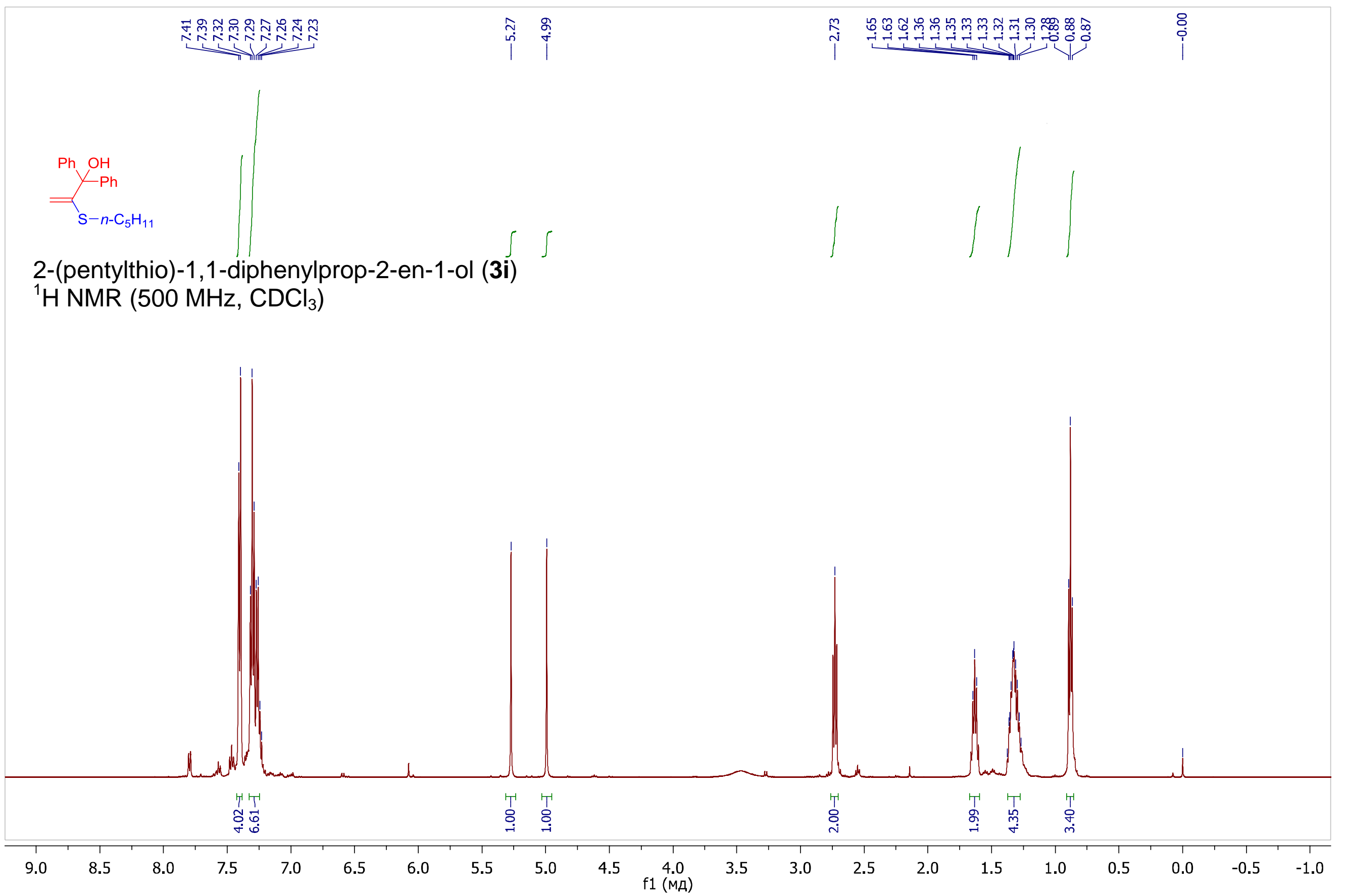




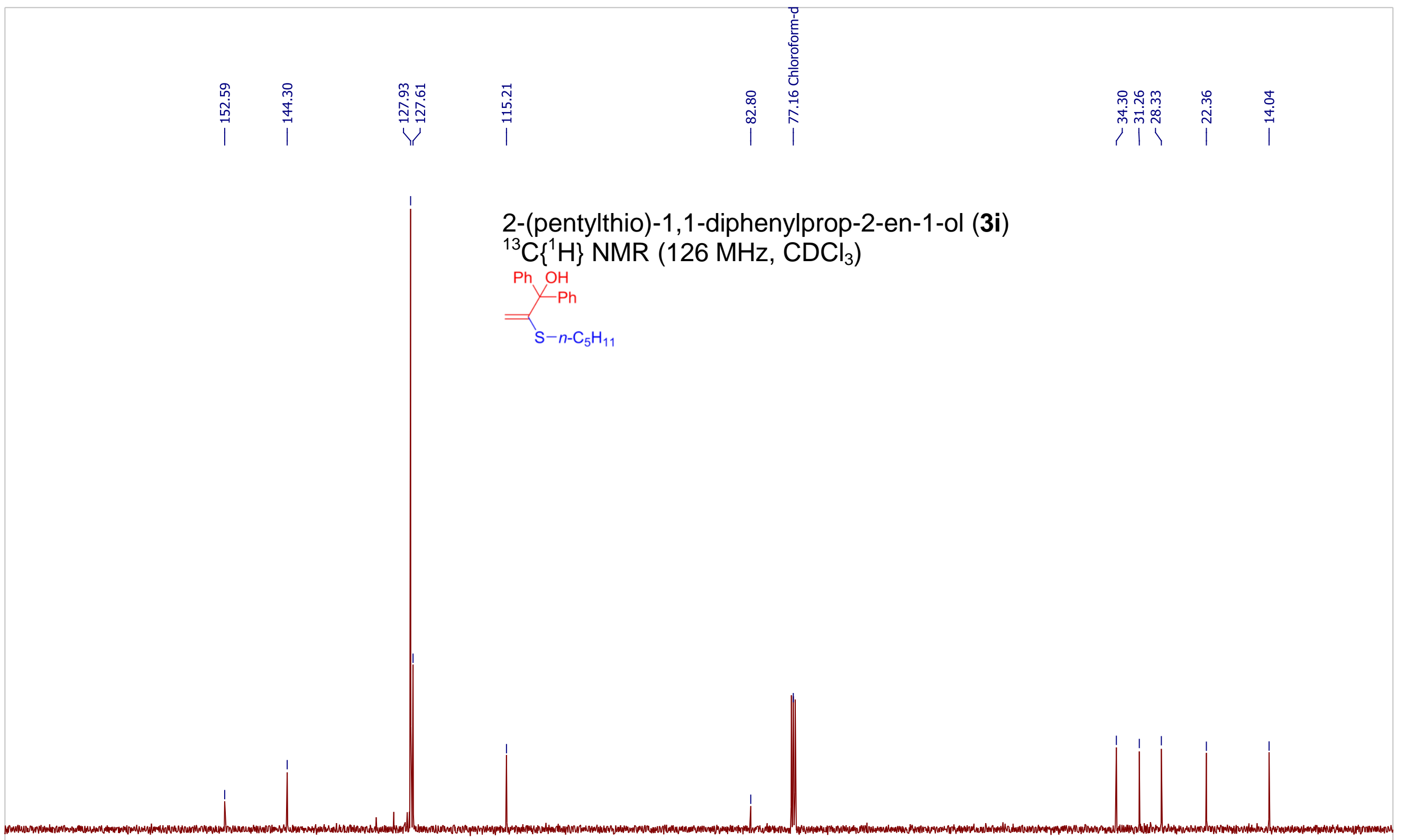

\begin{tabular}{|c|c|c|c|c|c|c|c|c|c|c|c|c|c|c|c|c|c|}
\hline \multirow{2}{*}{${ }_{180}$} & 1 & 1 & 1 & $T$ & 1 & $T$ & 1 & 1 & 1 & $T$ & $T$ & $T$ & $T$ & $T$ & $T$ & $T$ & $T$ \\
\hline & 170 & 160 & 150 & 140 & 130 & 120 & 110 & 100 & $\begin{array}{c}90 \\
\mathrm{ppm}\end{array}$ & 80 & 70 & 60 & 50 & 40 & 30 & 20 & 10 \\
\hline
\end{tabular}


4-(pentylthio)pent-4-en-2-ol (3j)

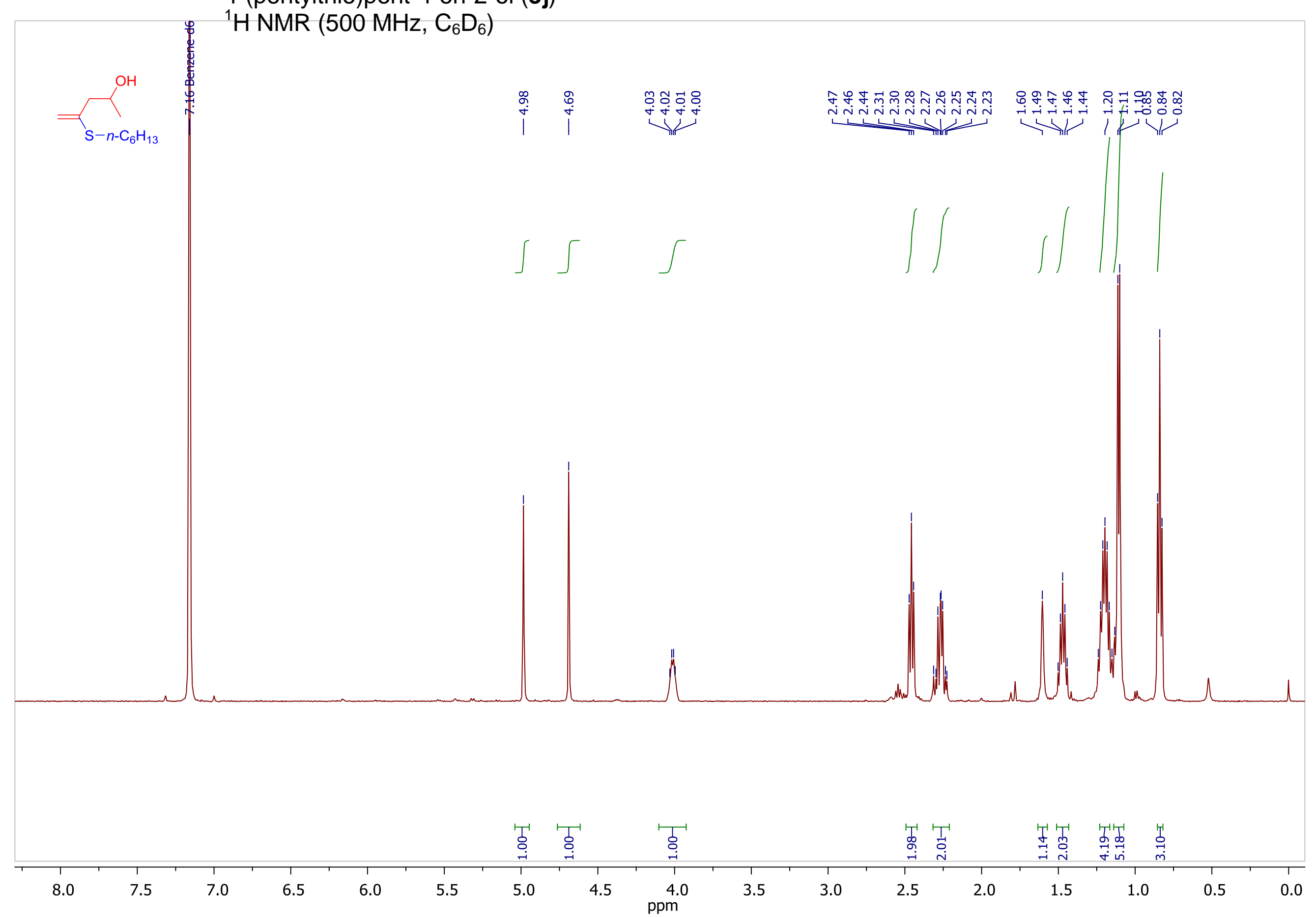




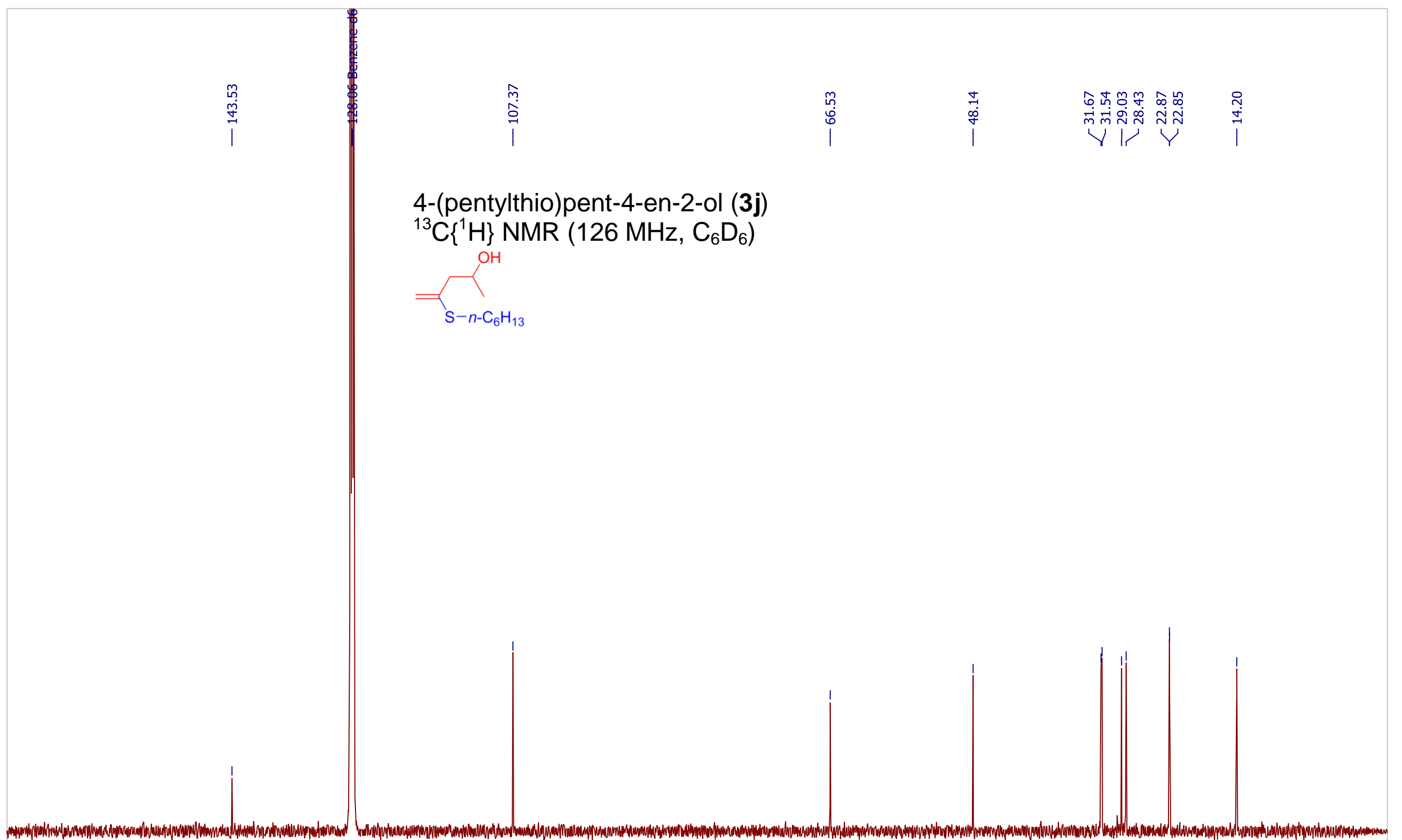

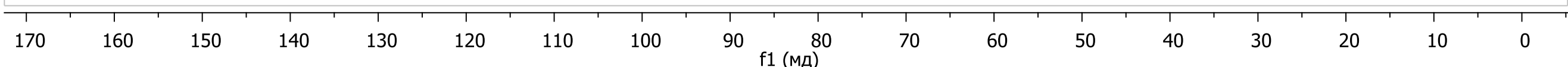




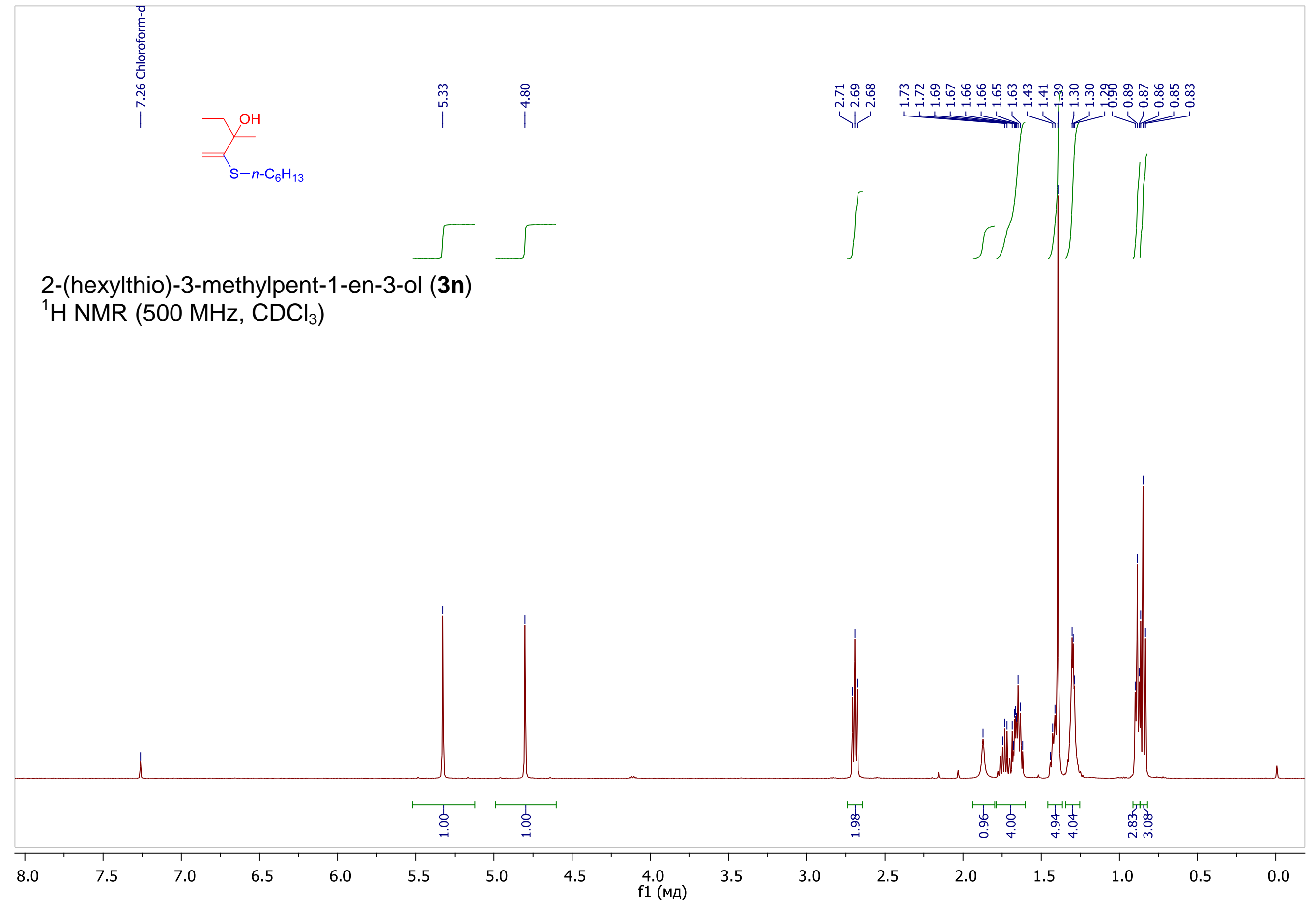


2-(hexylthio)-3-methylpent-1-en-3-ol (3n)

${ }^{13} \mathrm{C}\left\{{ }^{1} \mathrm{H}\right\}$ NMR $\left(126 \mathrm{MHz}, \mathrm{CDCl}_{3}\right)$
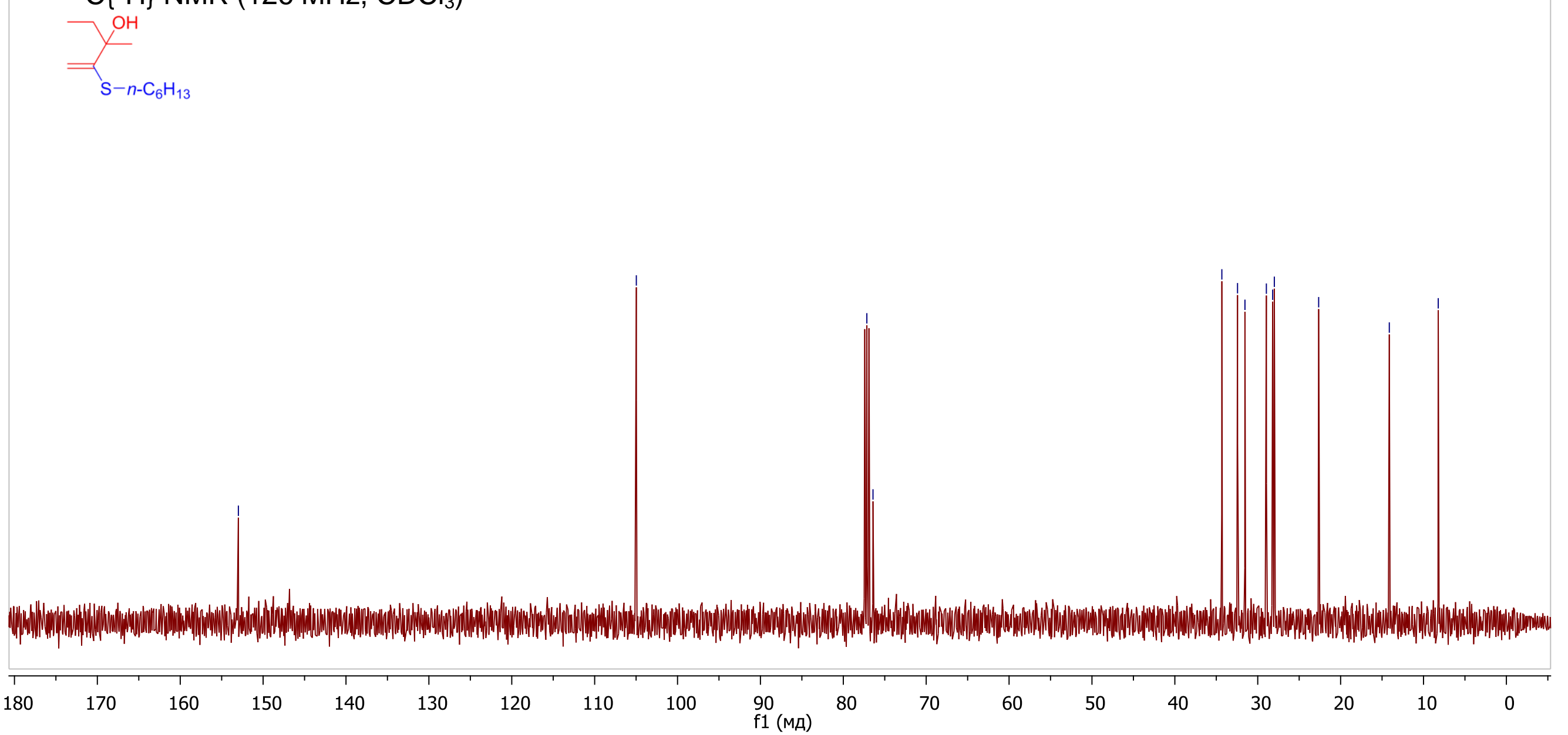


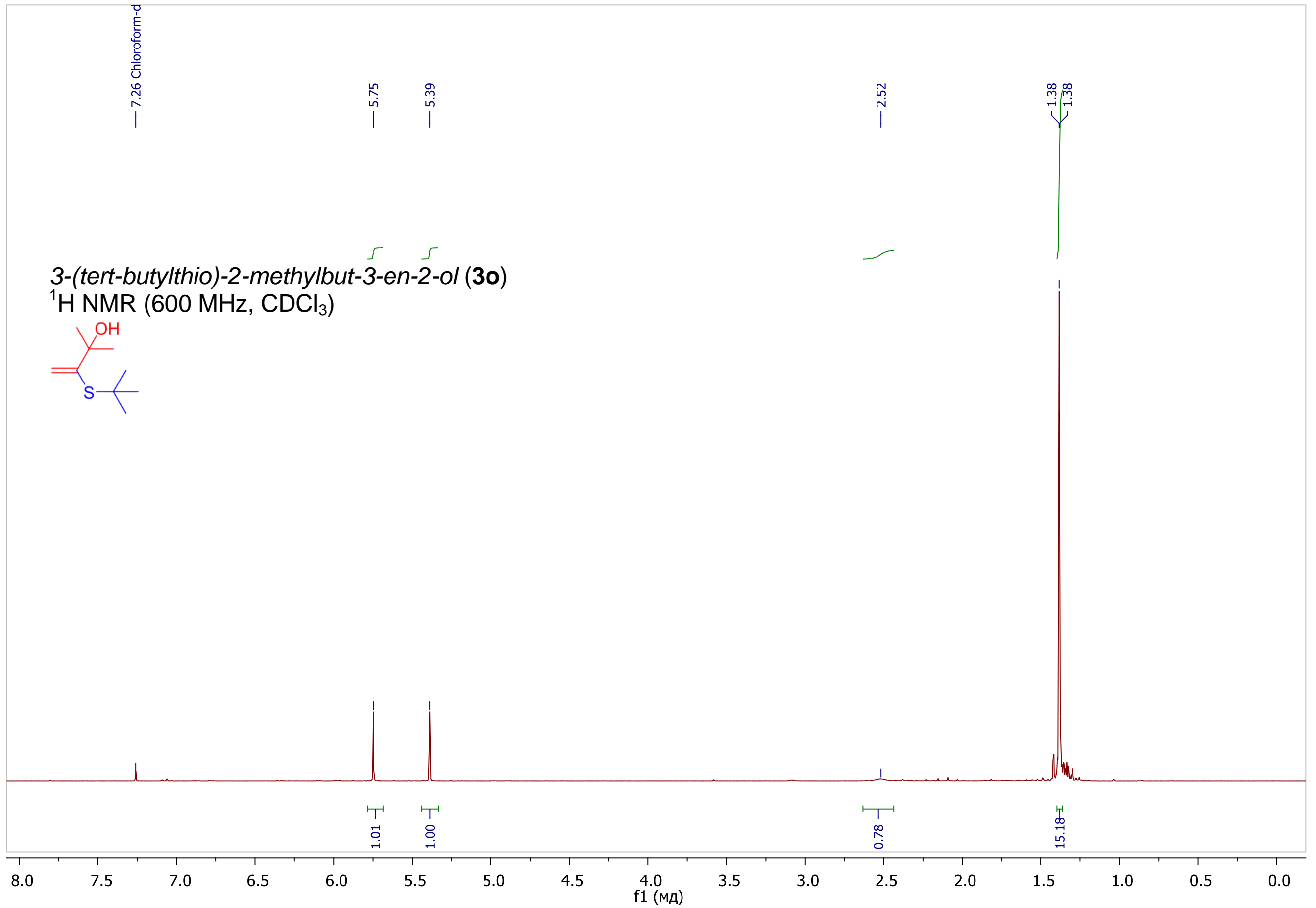




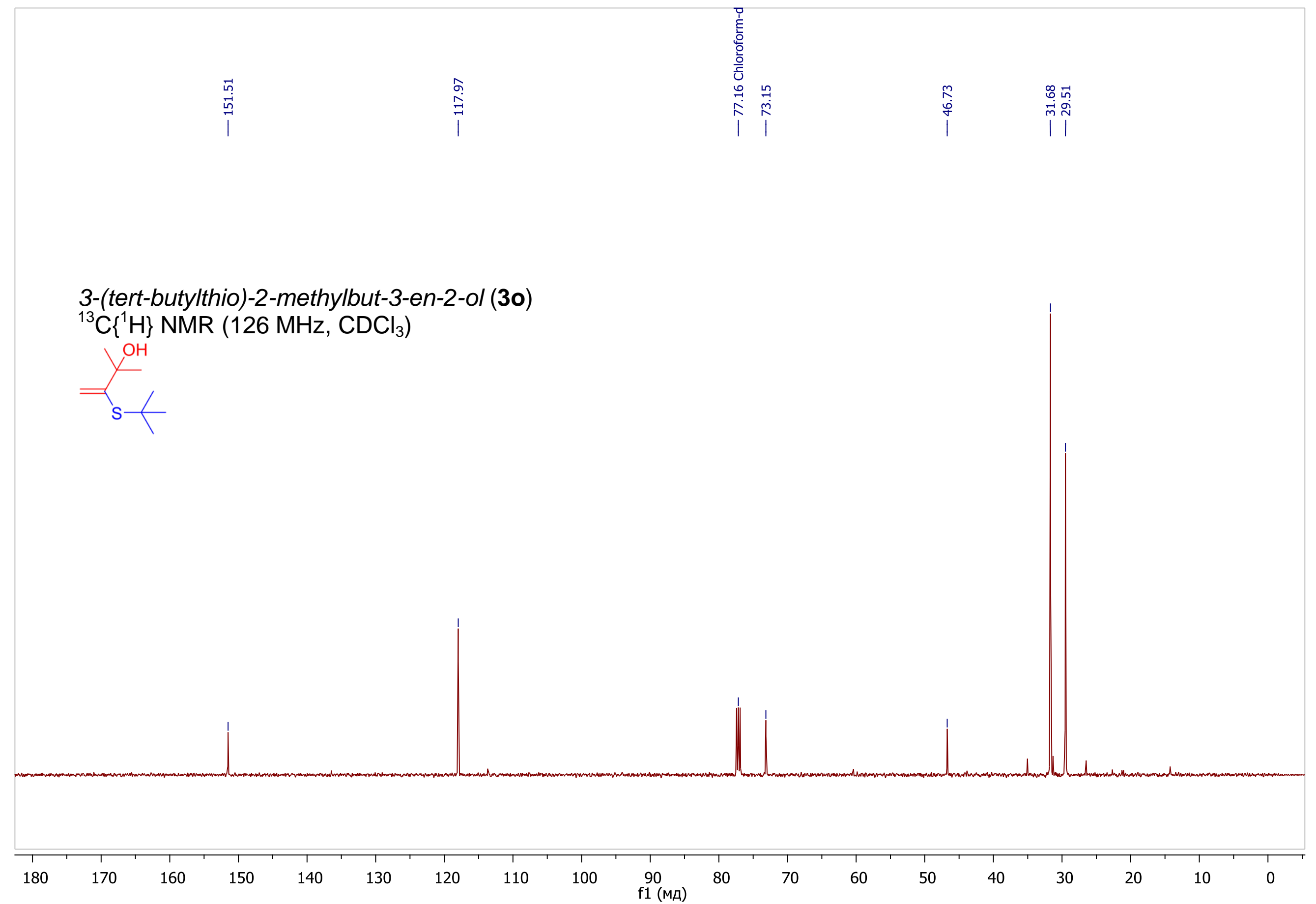




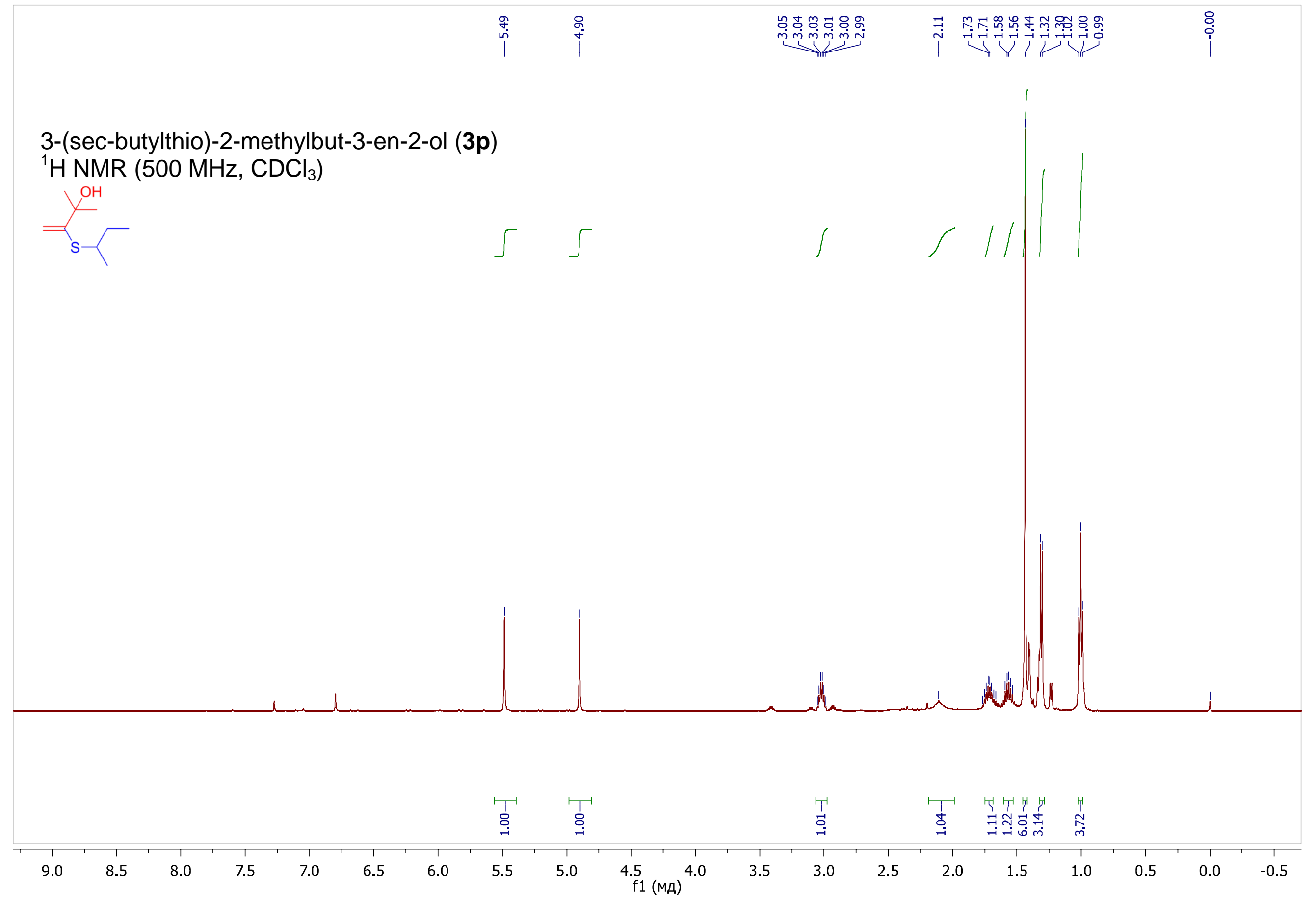




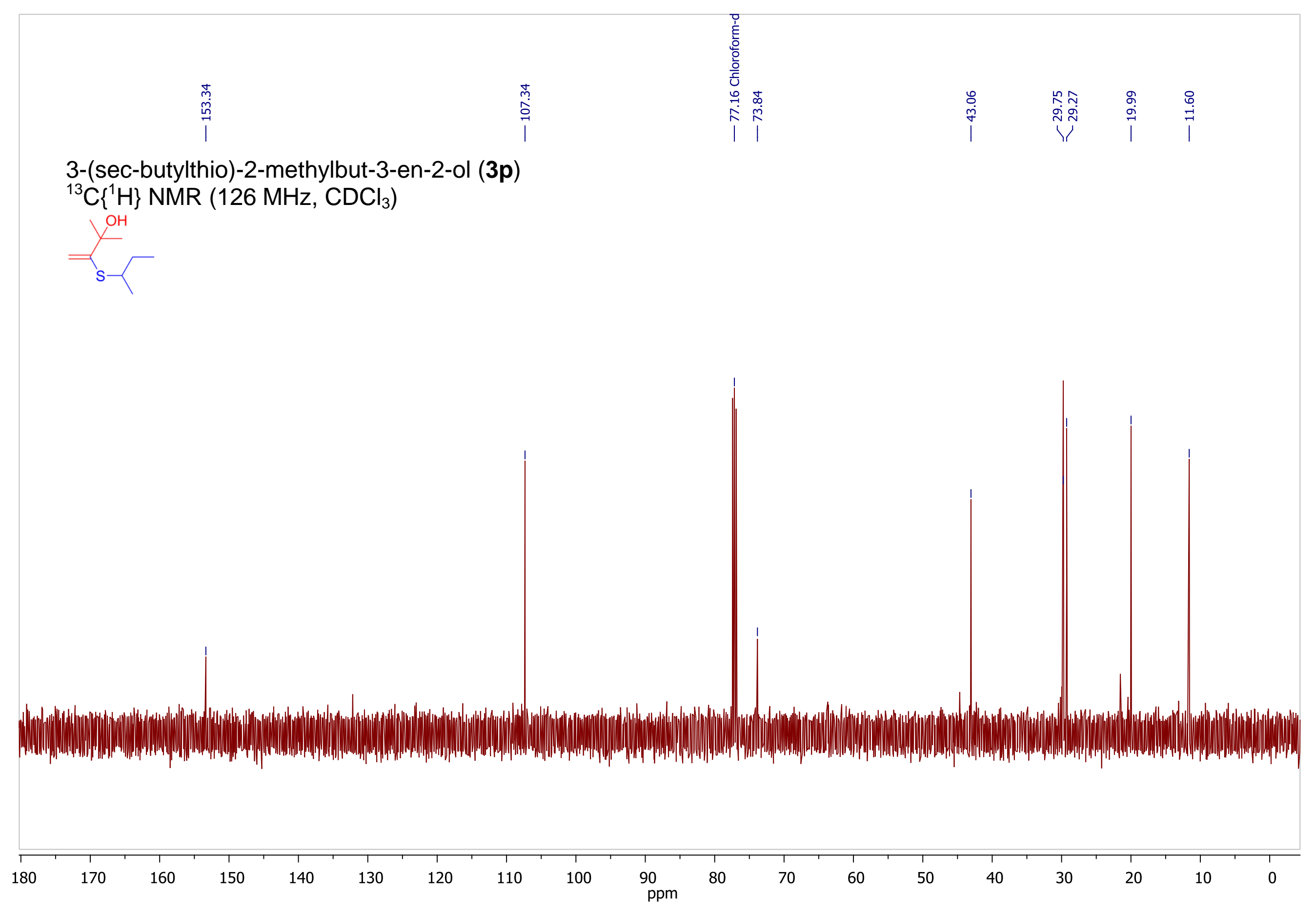




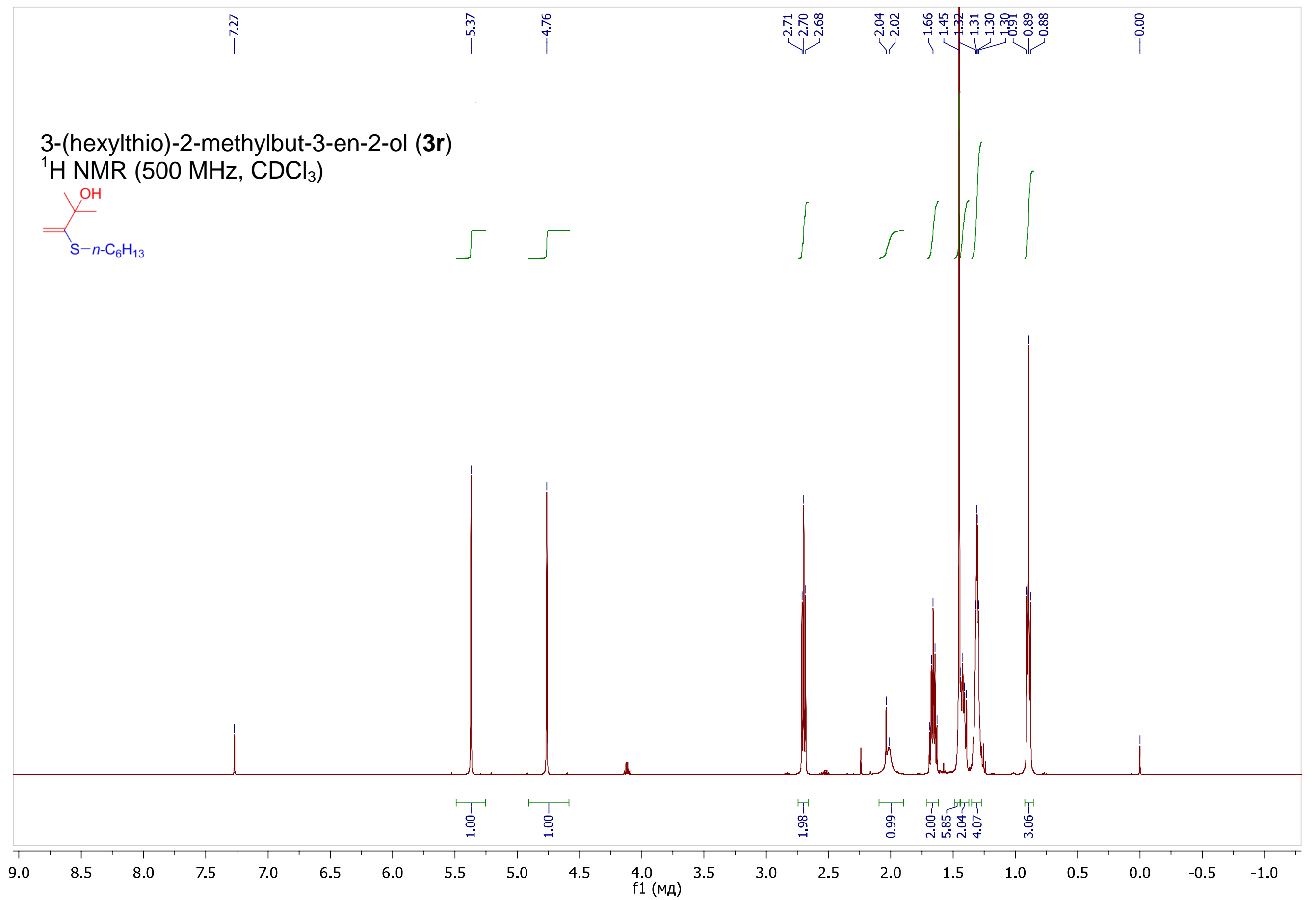


3-(hexylthio)-2-methylbut-3-en-2-ol (3r)

${ }^{13} \mathrm{C}\left\{{ }^{1} \mathrm{H}\right\} \mathrm{NMR}\left(126 \mathrm{MHz}, \mathrm{CDCl}_{3}\right)$

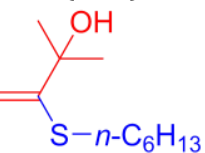

| 


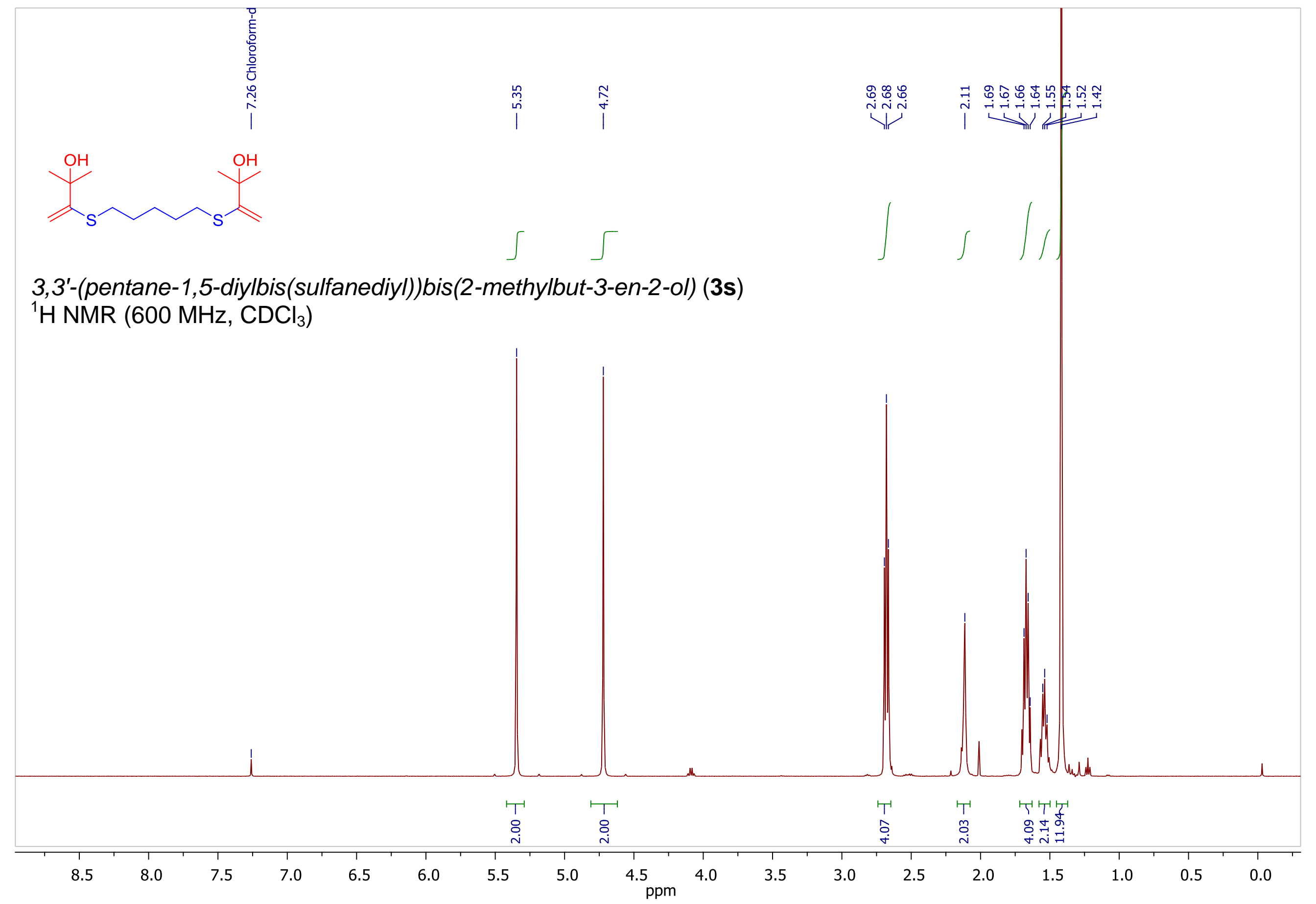




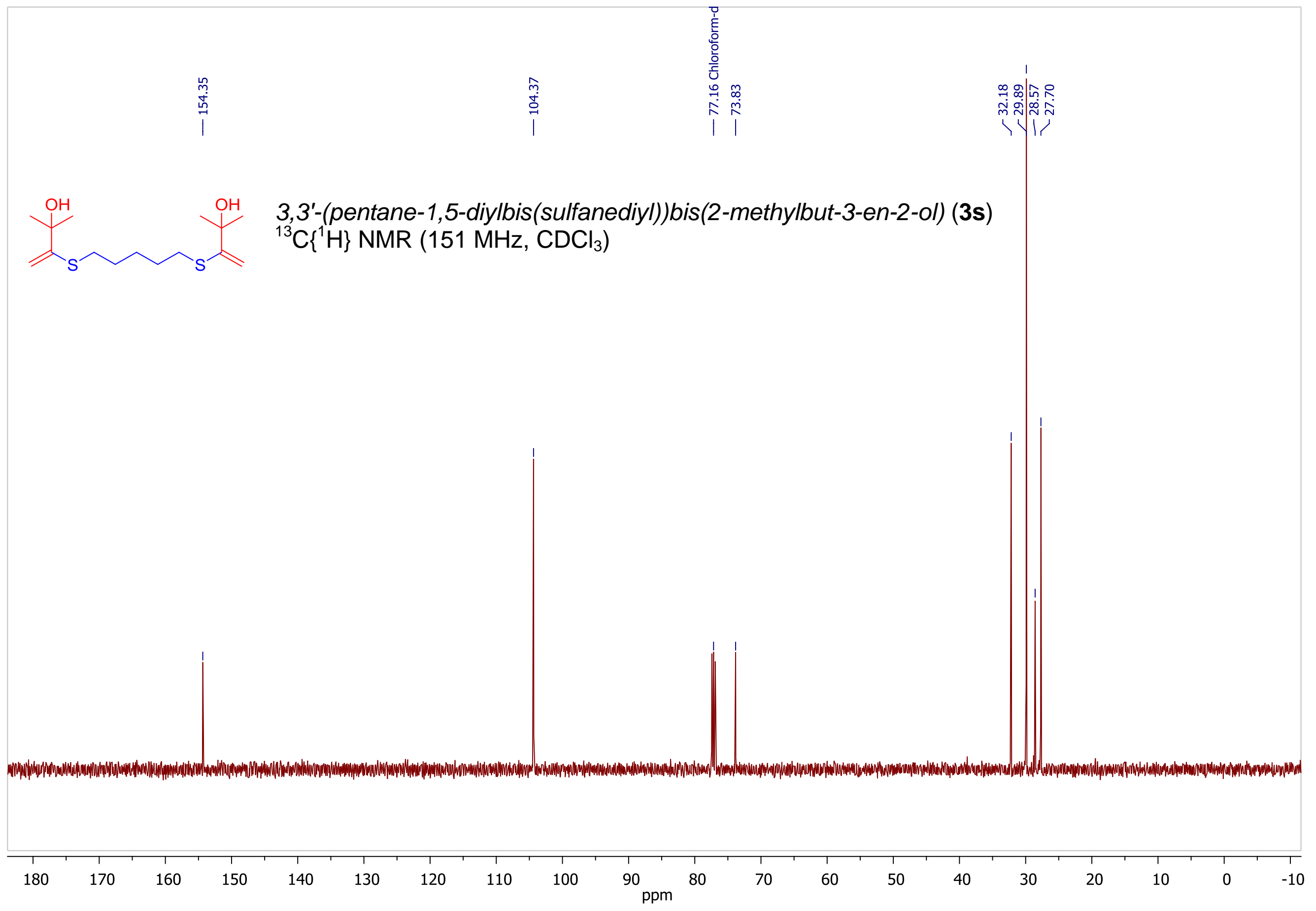




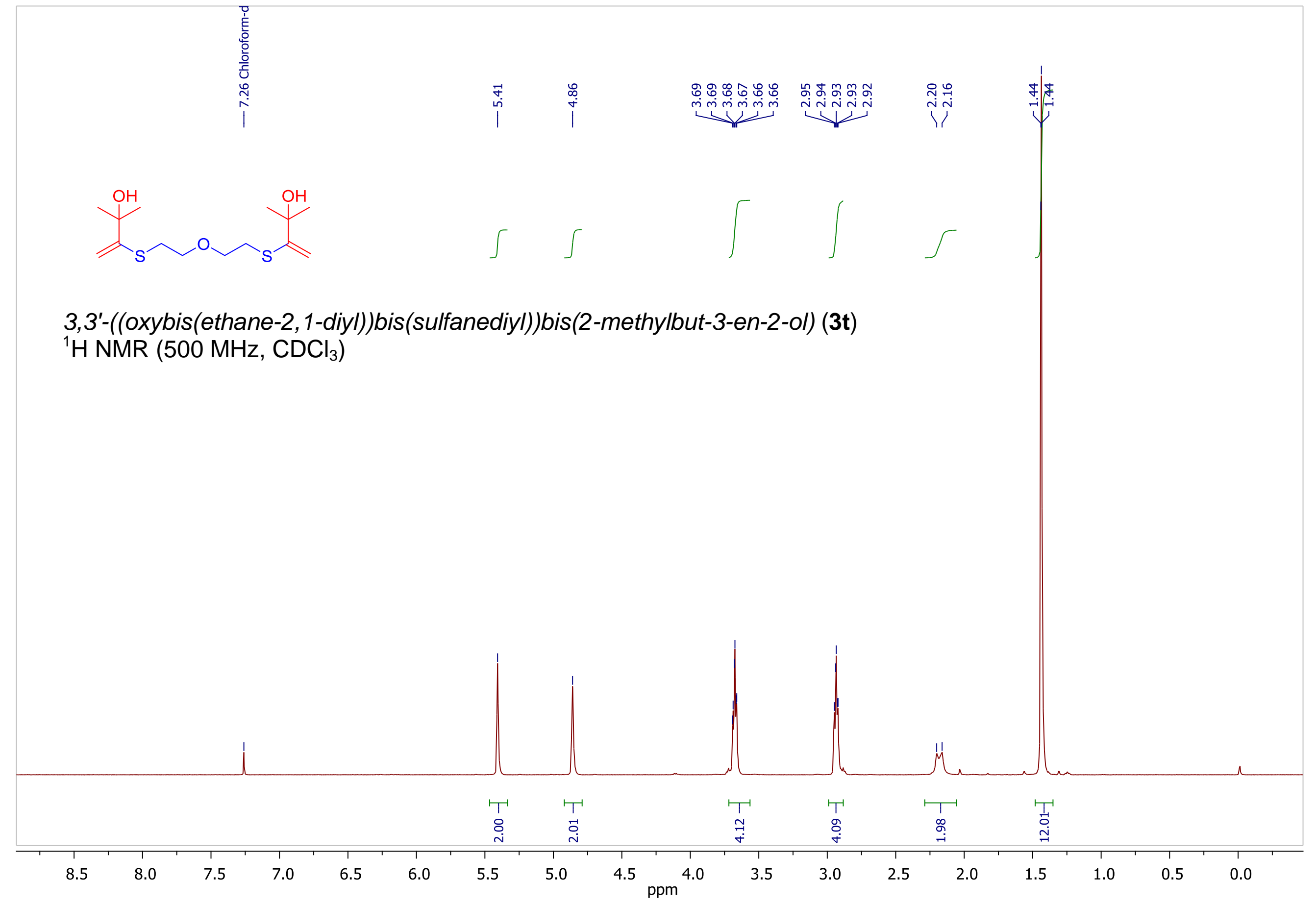




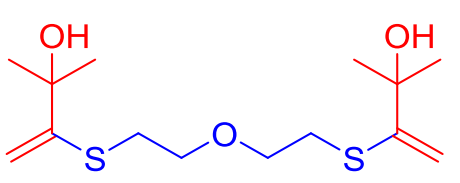

3,3'-((oxybis(ethane-2,1-diyl))bis(sulfanediyl))bis(2-methylbut-3-en-2-ol) (3t) ${ }^{13} \mathrm{C}\left\{{ }^{1} \mathrm{H}\right\} \mathrm{NMR}\left(126 \mathrm{MHz}, \mathrm{CDCl}_{3}\right)$

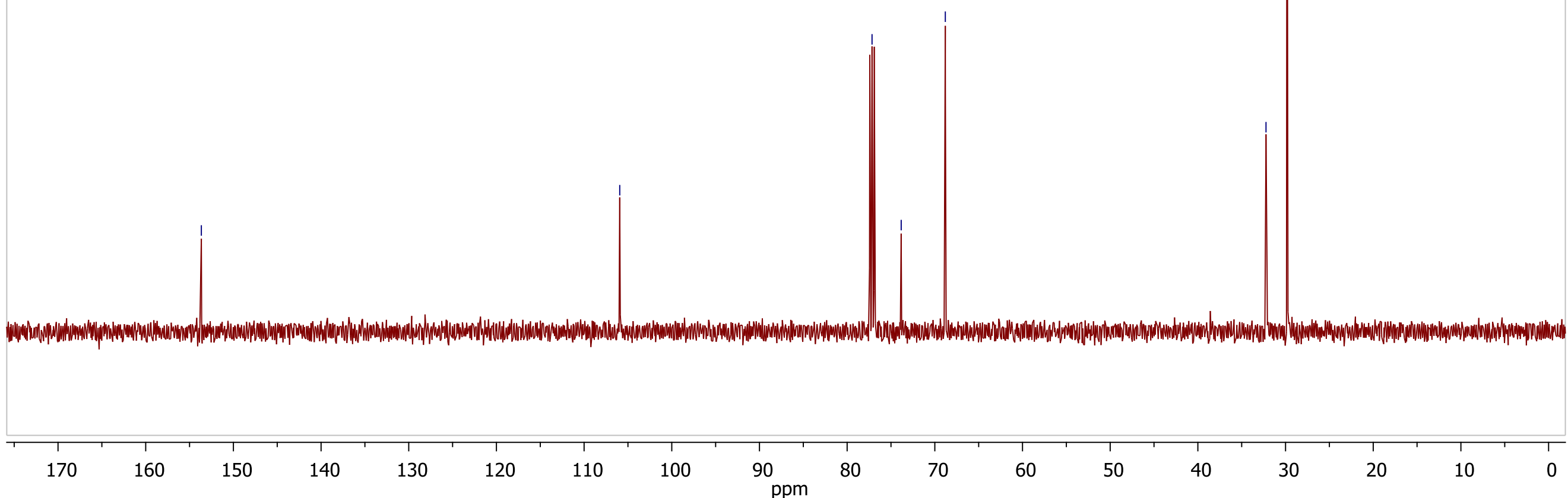




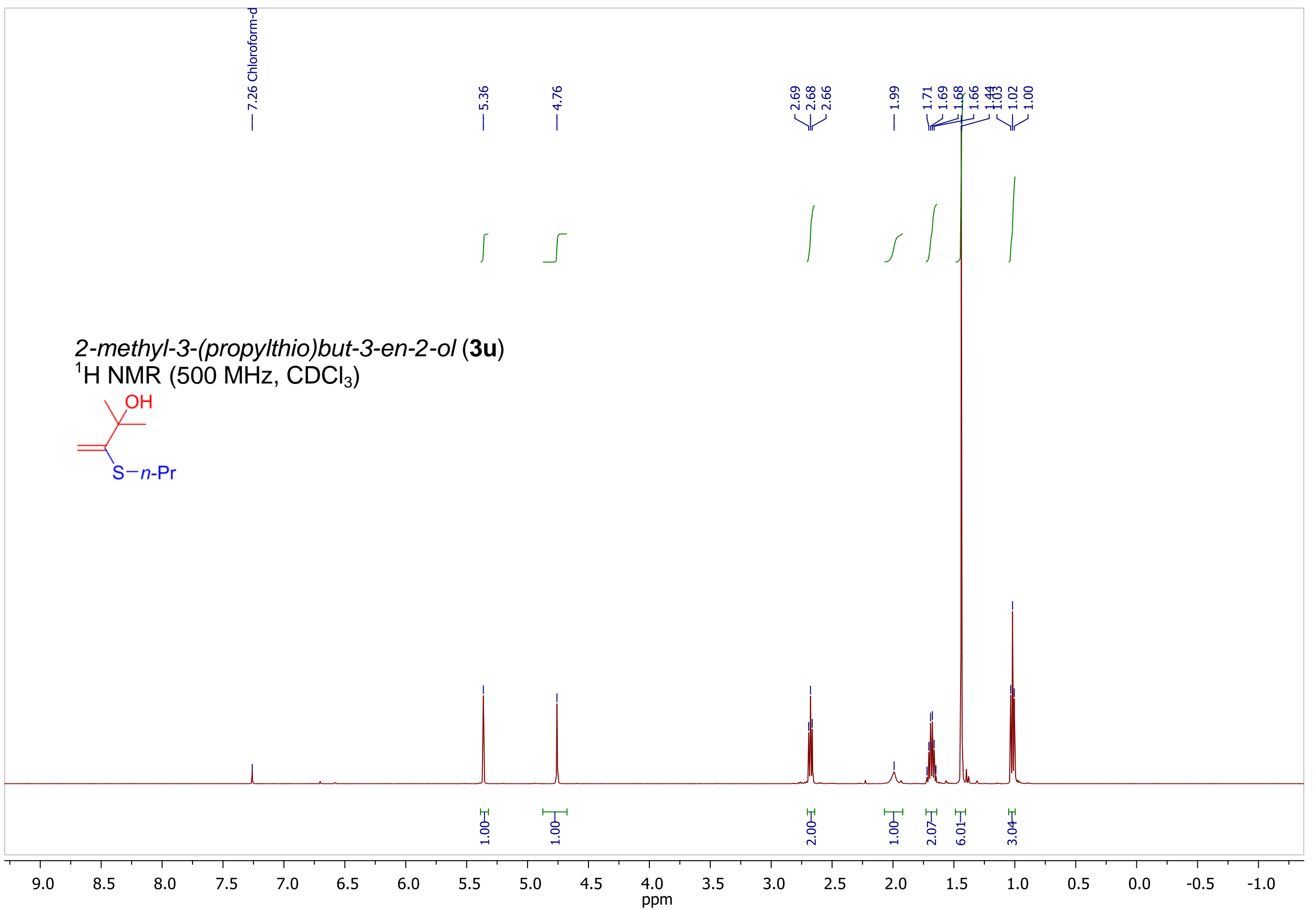


2-methyl-3-(propylthio)but-3-en-2-ol (3u)

${ }^{13} \mathrm{C}\left\{{ }^{1} \mathrm{H}\right\}$ NMR $\left(126 \mathrm{MHz}, \mathrm{CDCl}_{3}\right)$
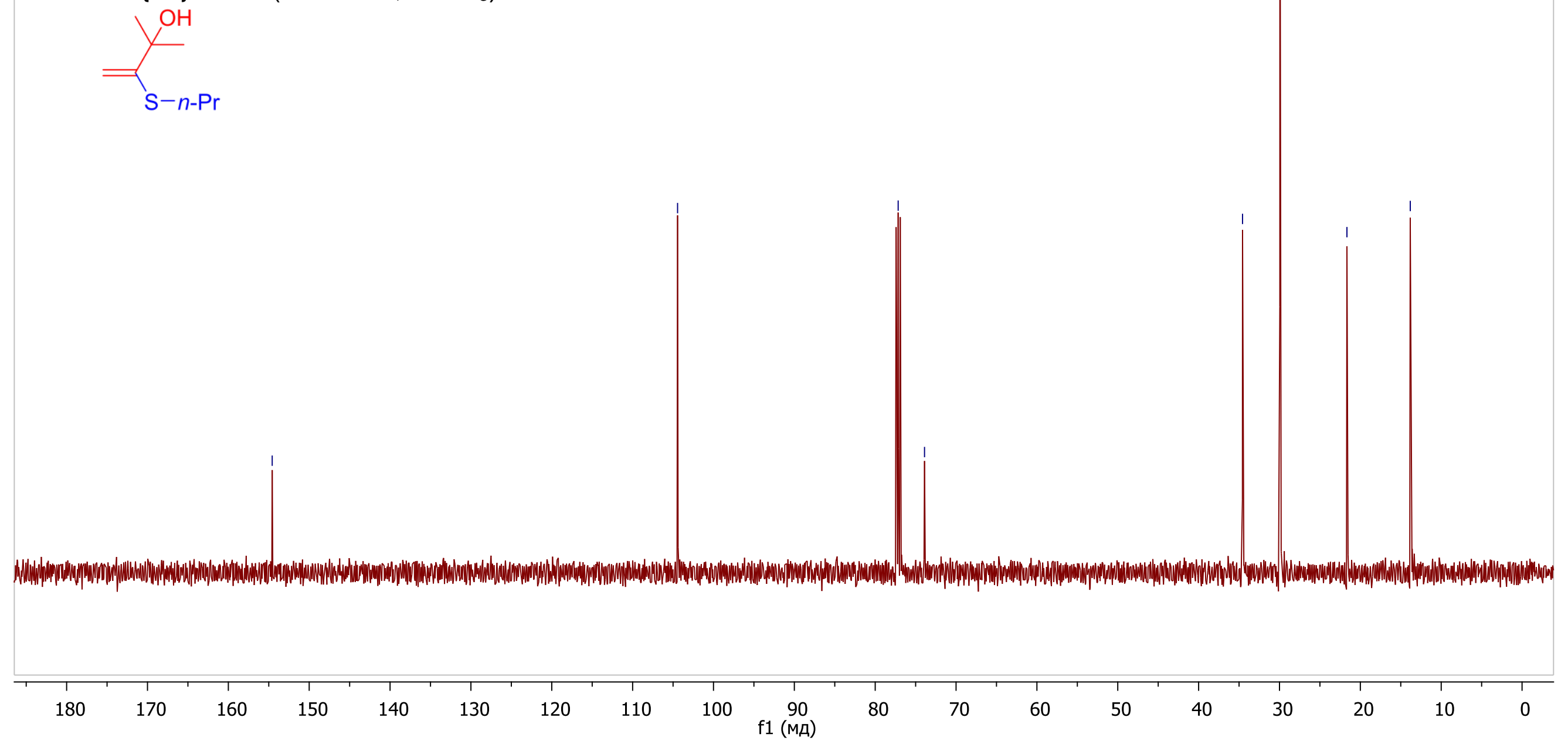

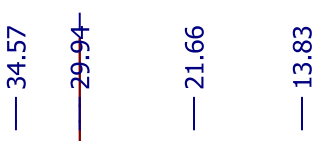




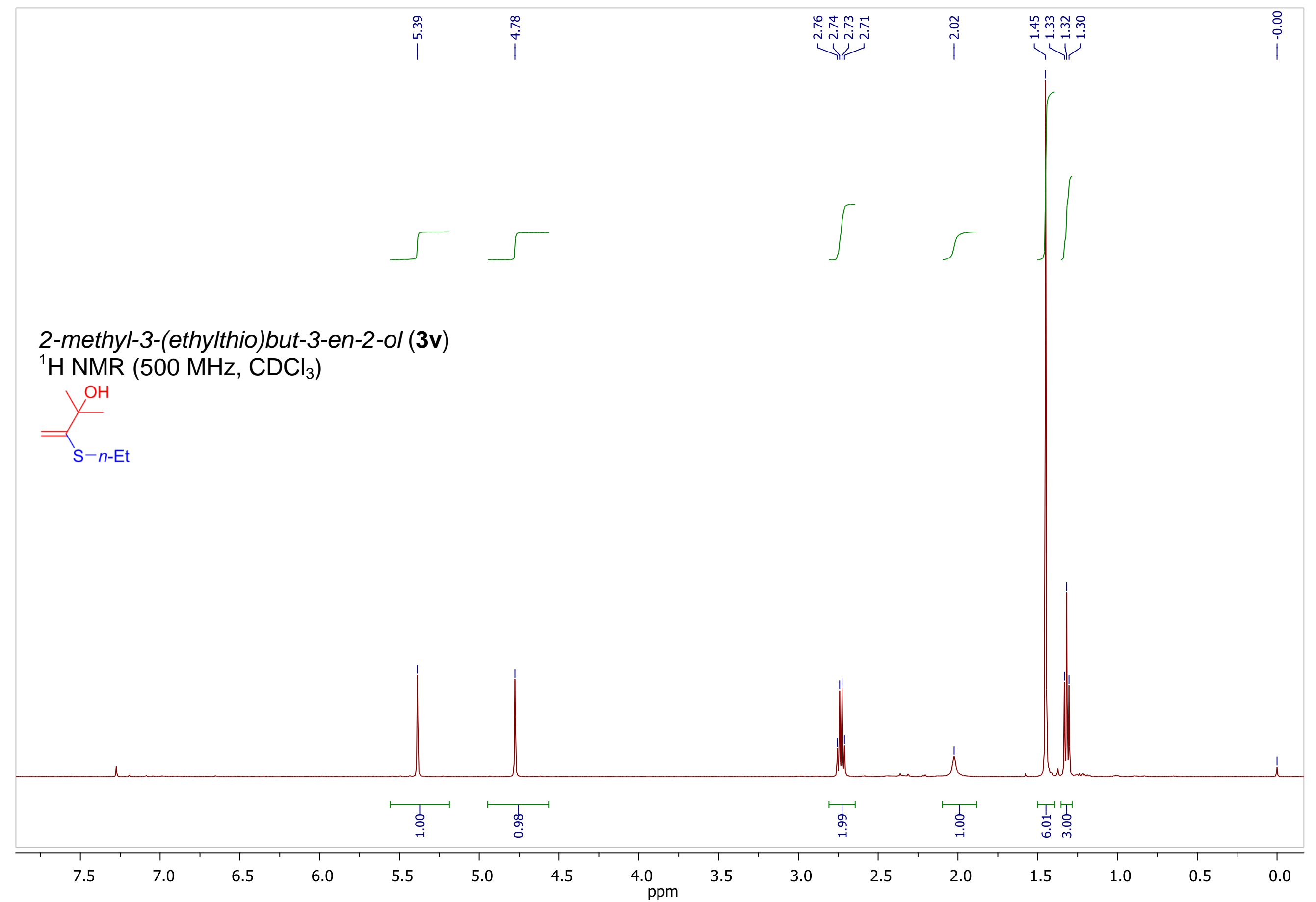


2-methyl-3-(ethylthio)but-3-en-2-ol (3v)

${ }^{13} \mathrm{C}\left\{{ }^{1} \mathrm{H}\right\} \mathrm{NMR}\left(151 \mathrm{MHz}, \mathrm{CDCl}_{3}\right)$
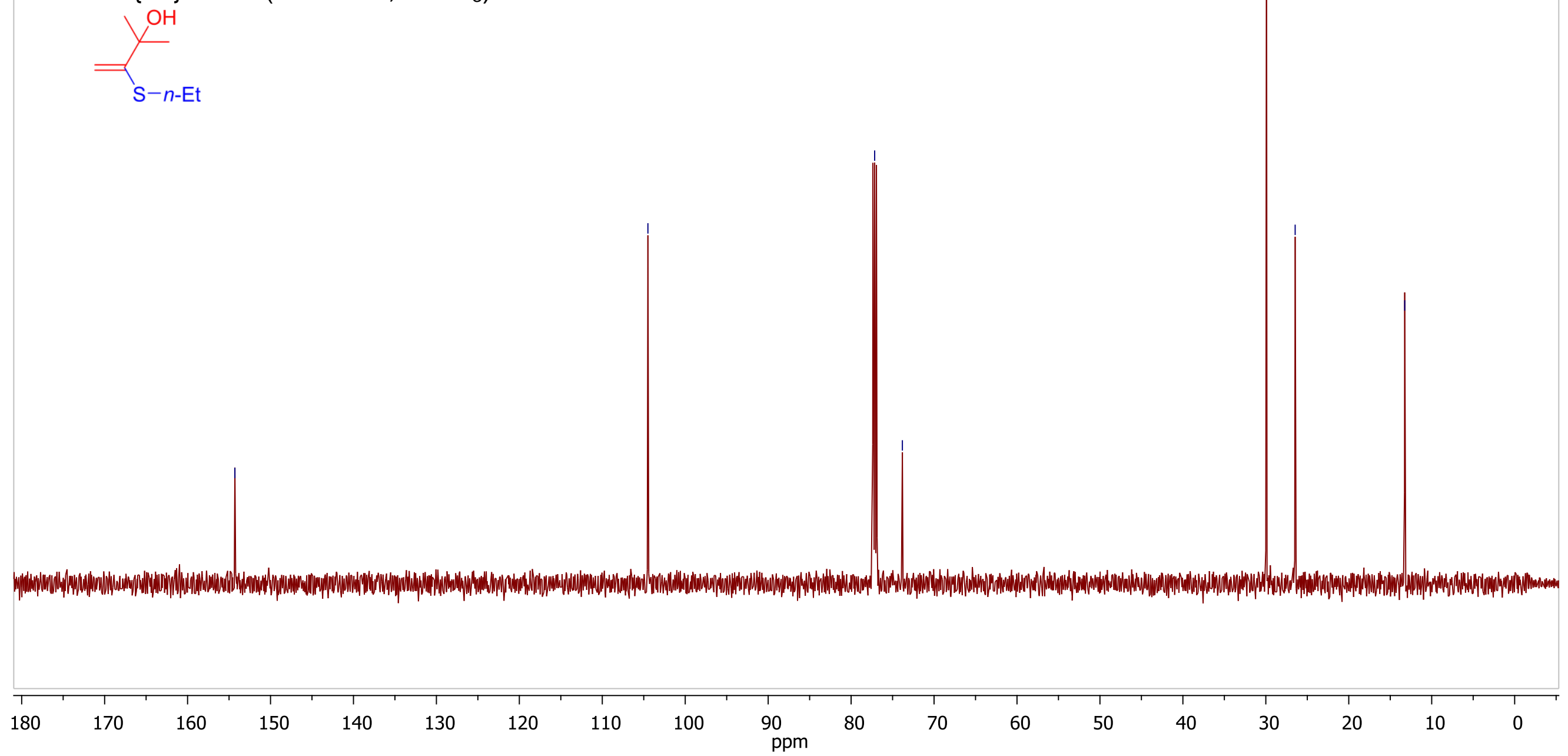

$-n-\mathrm{Et}$

2.

ก

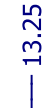




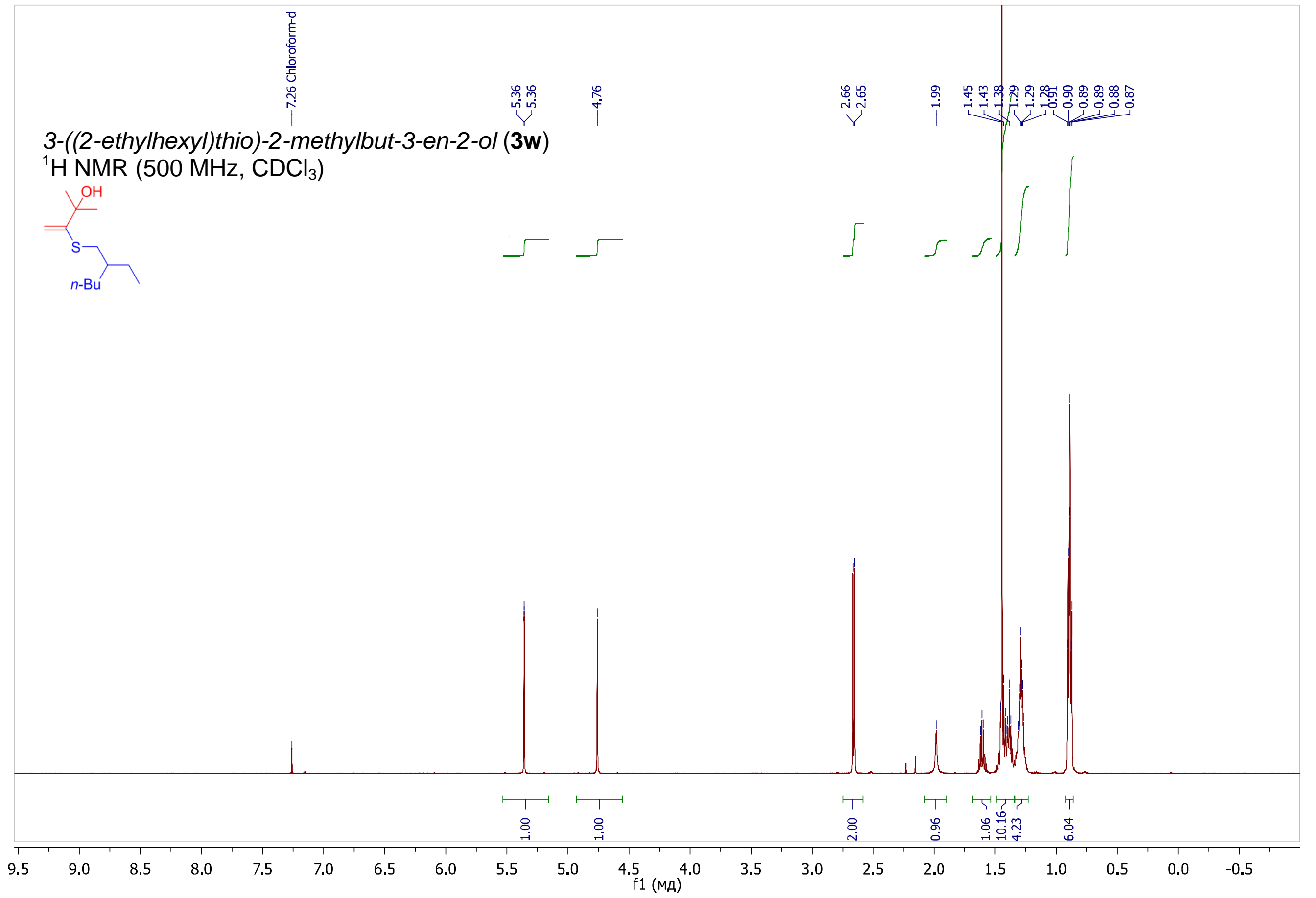




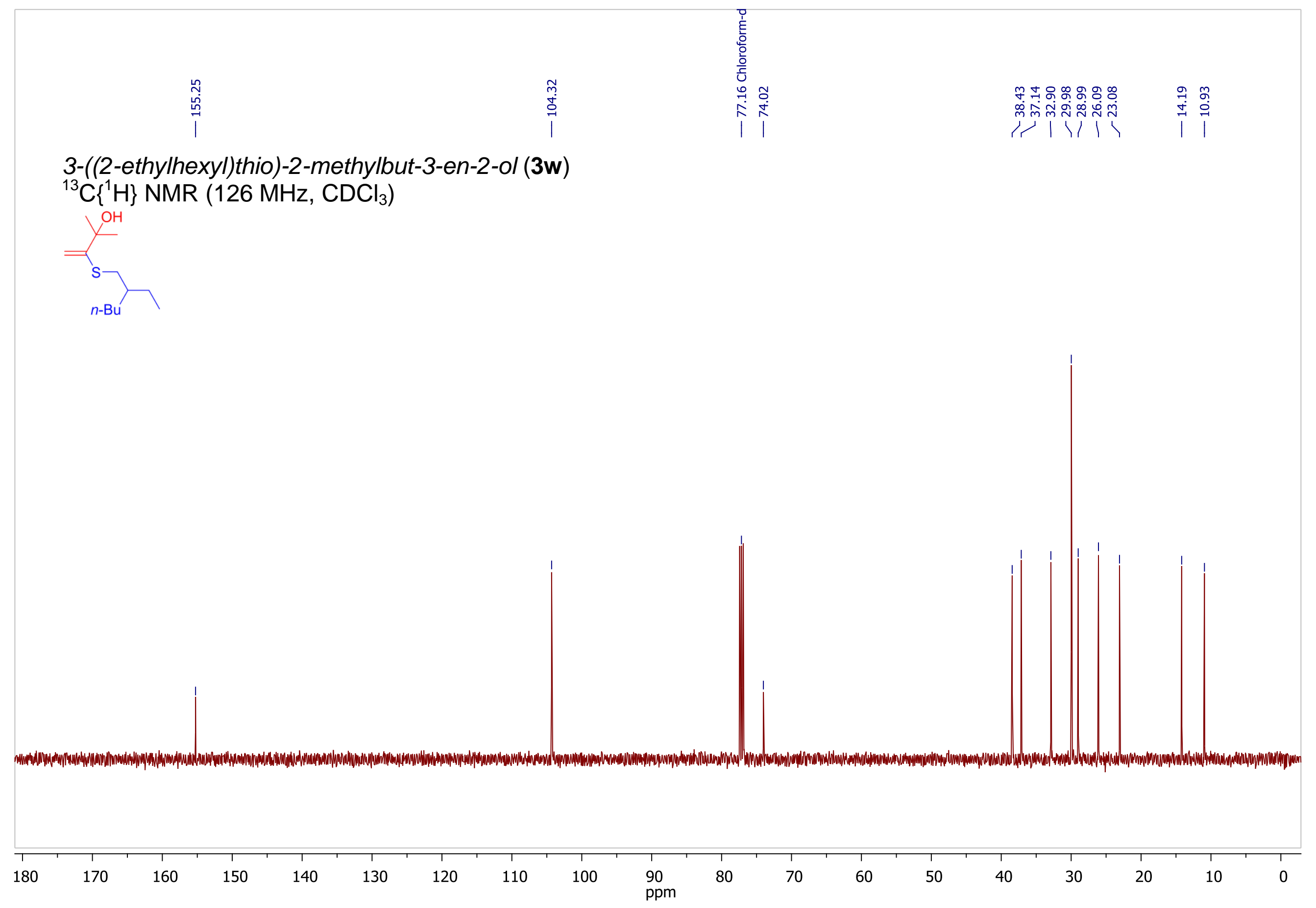




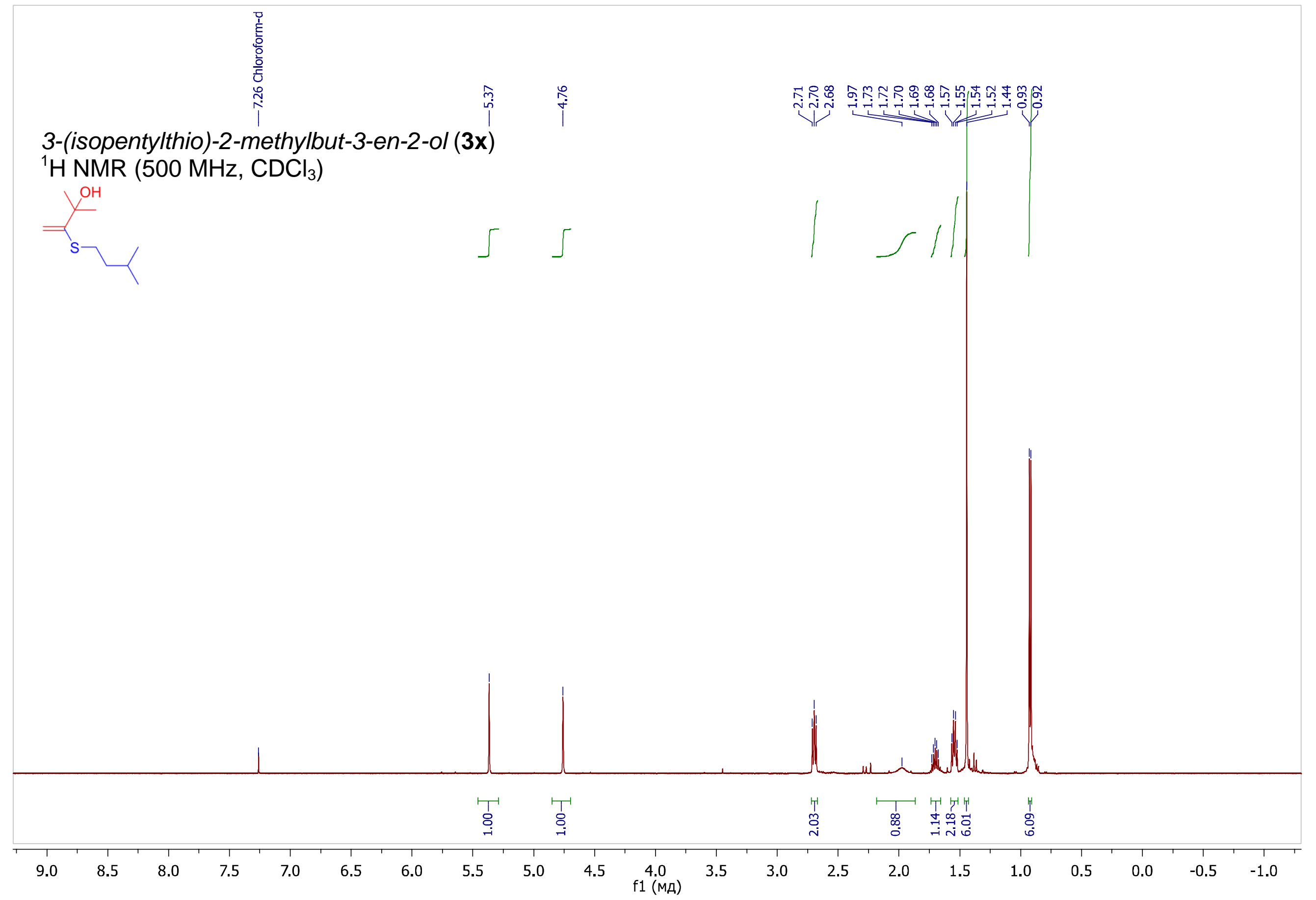




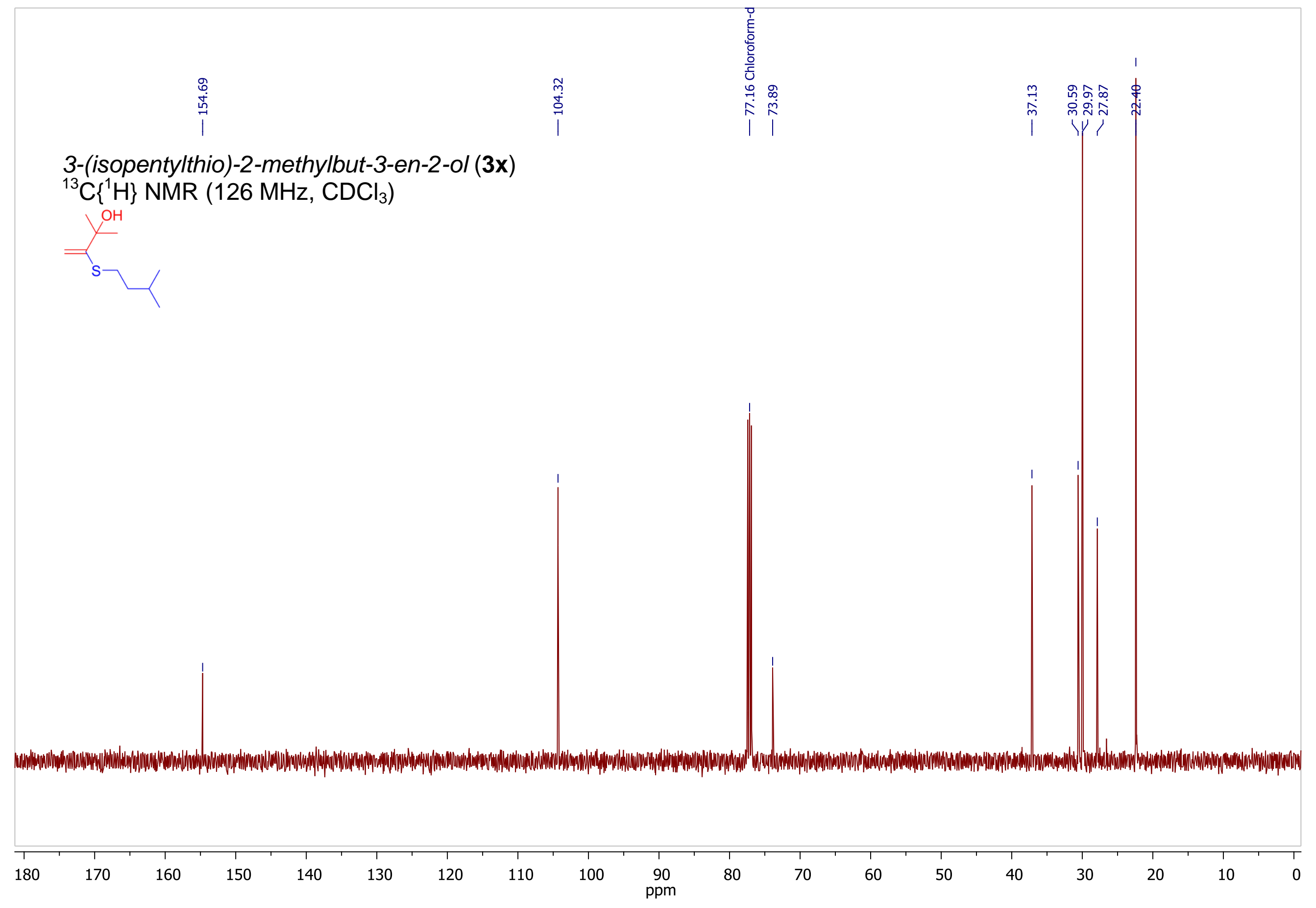




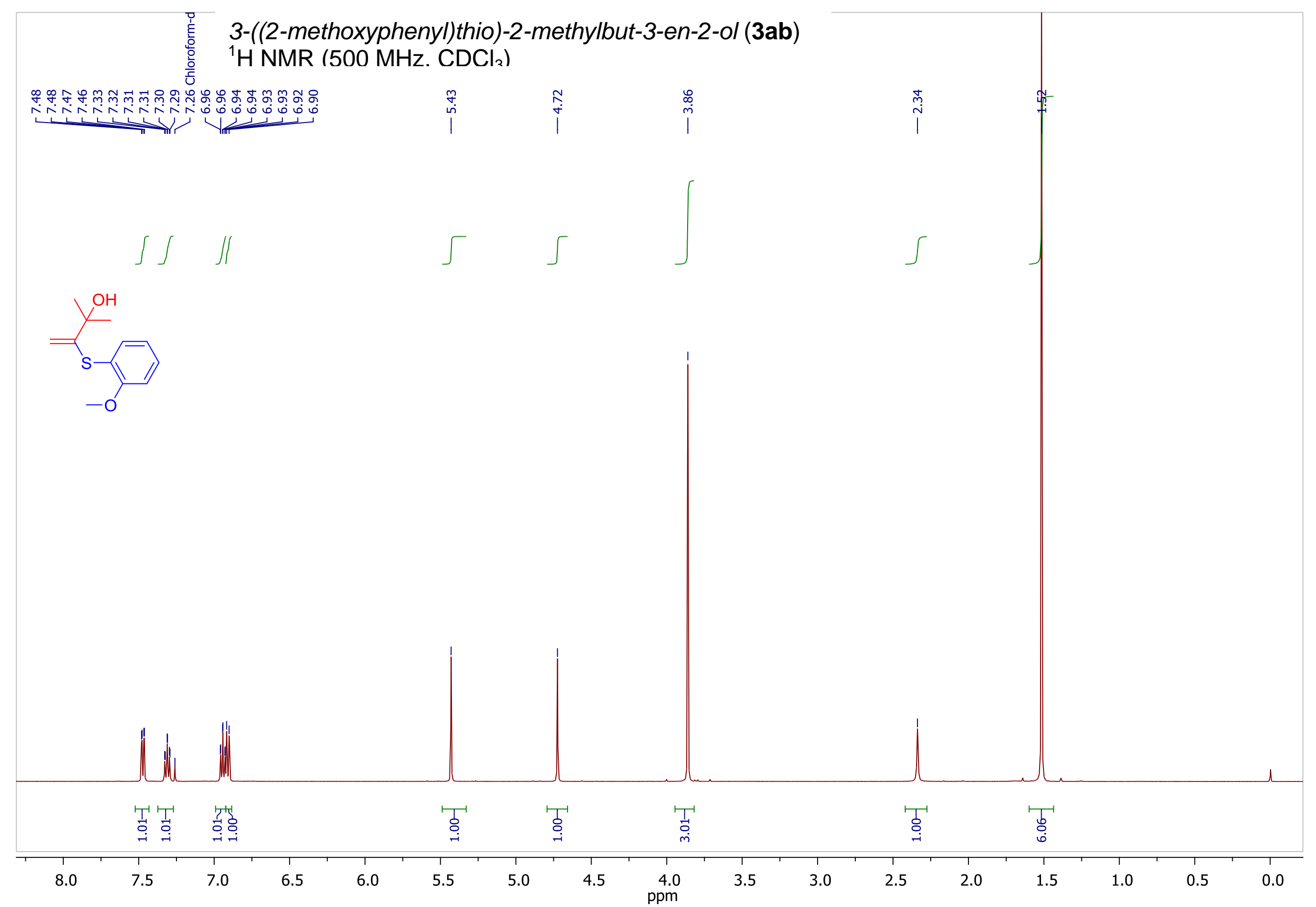




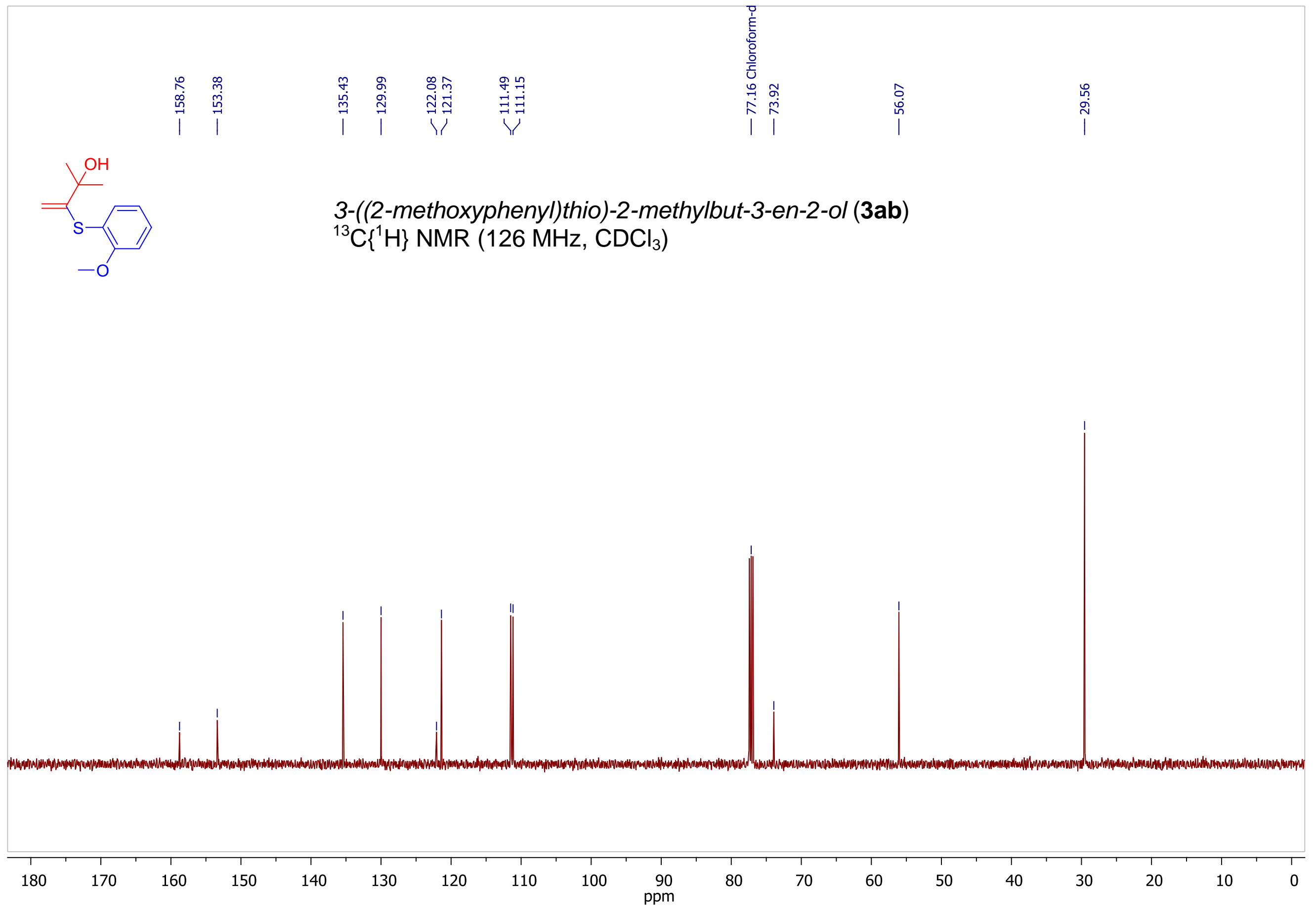




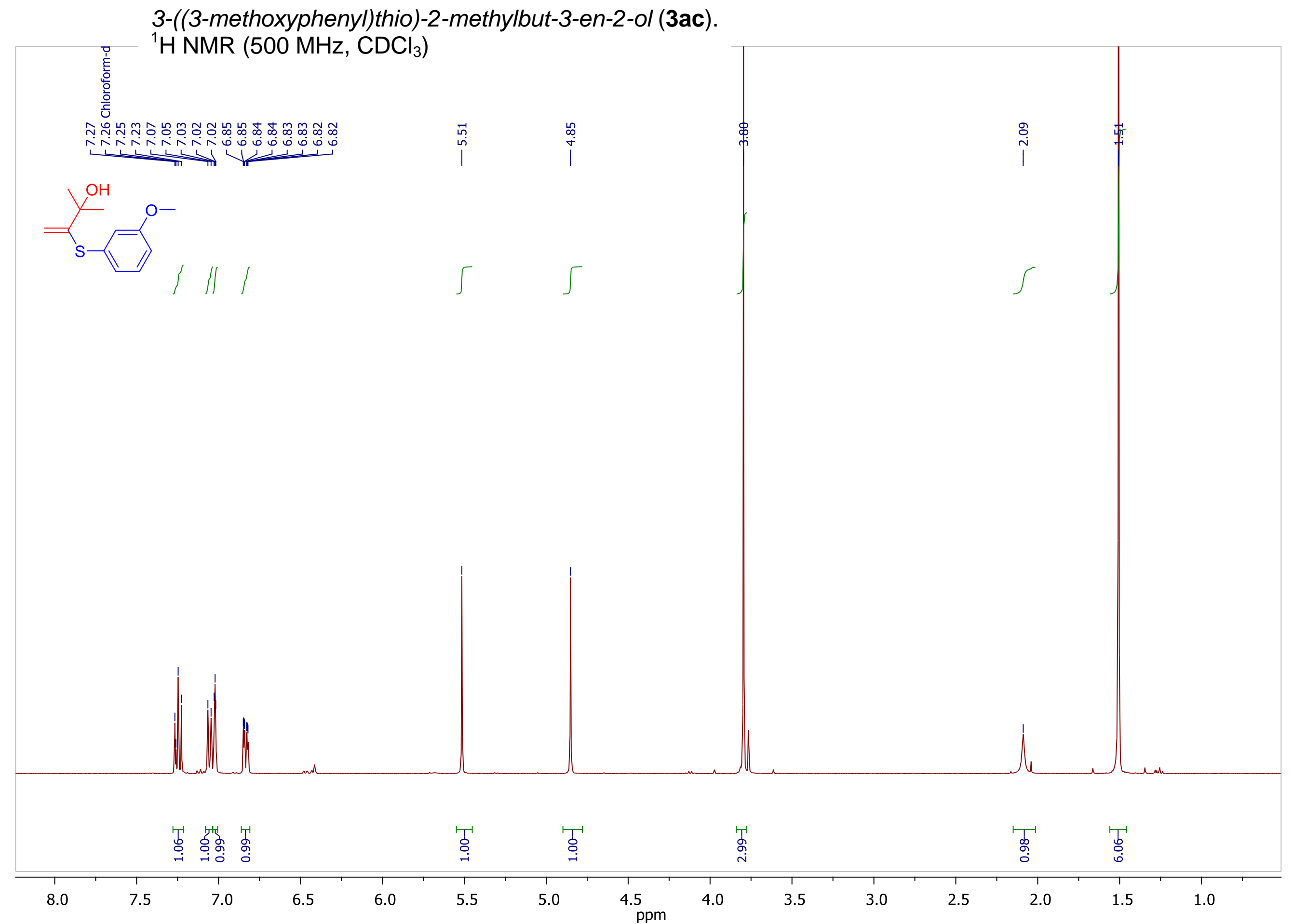


3-((3-methoxyphenyl)thio)-2-methylbut-3-en-2-ol (3ac). ${ }^{13} \mathrm{C}\left\{{ }^{1} \mathrm{H}\right\}$ NMR $\left(126 \mathrm{MHz}, \mathrm{CDCl}_{3}\right)$

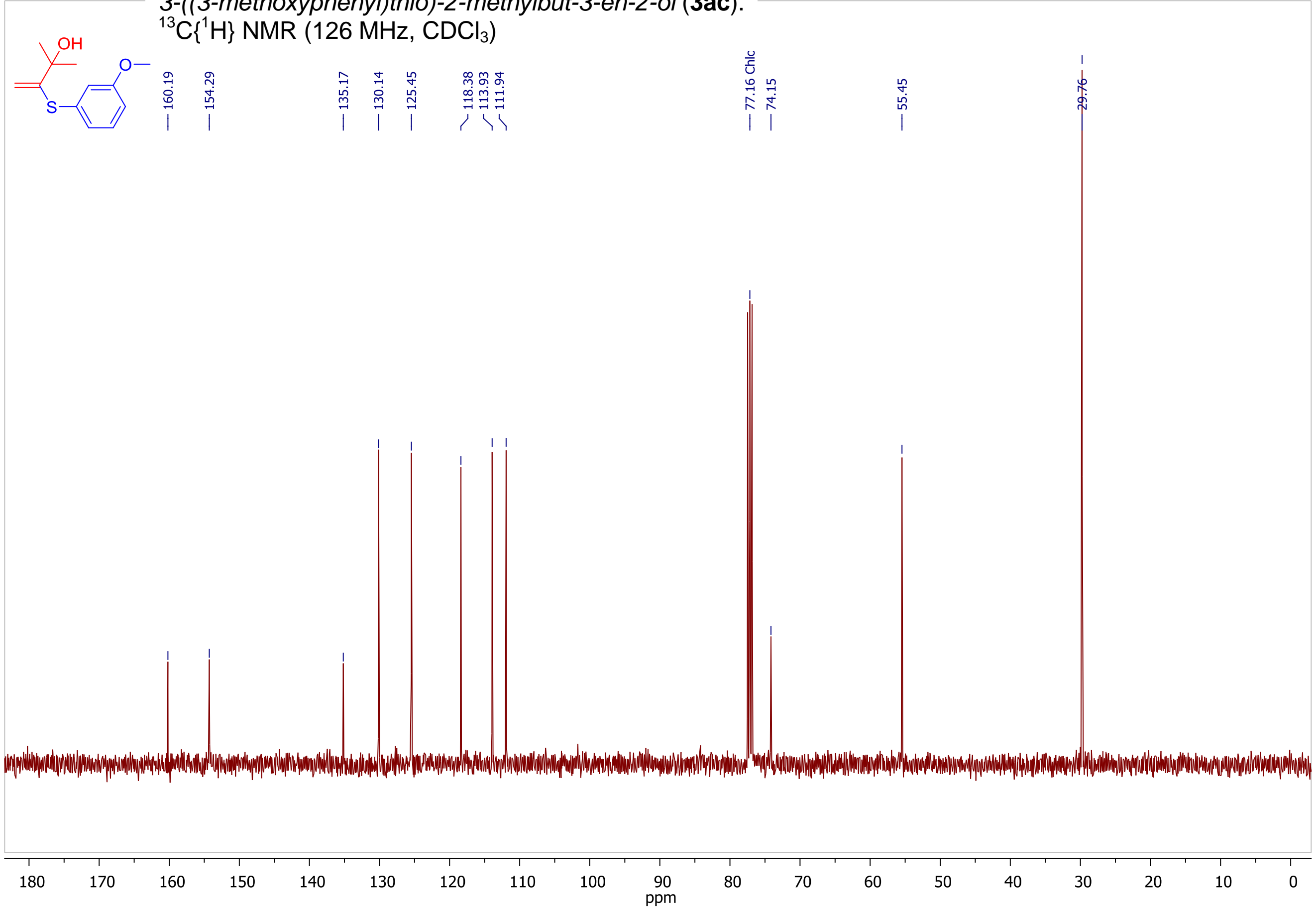


3-((4-methoxyphenyl)thio)-2-methylbut-3-en-2-ol (3ad).

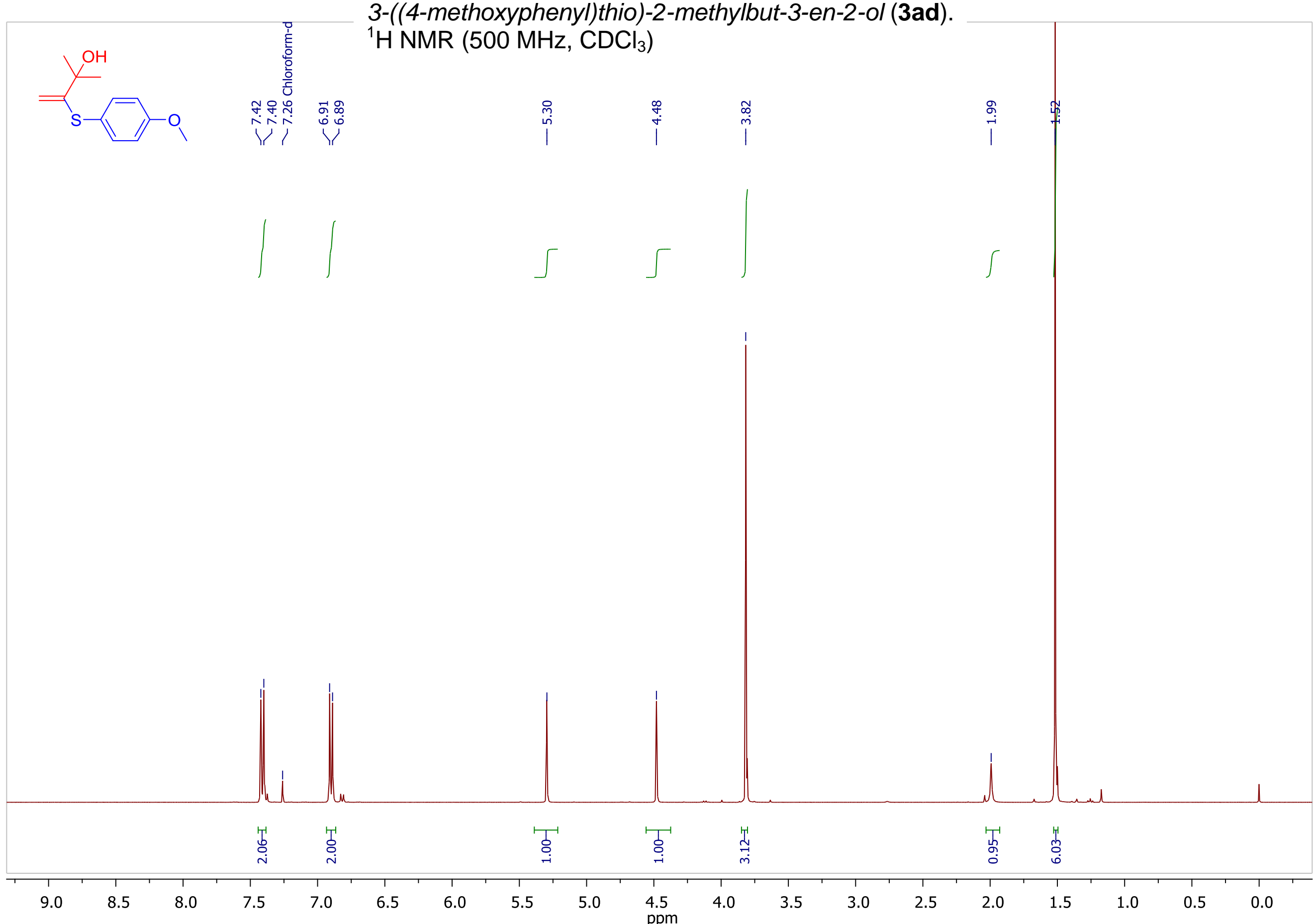




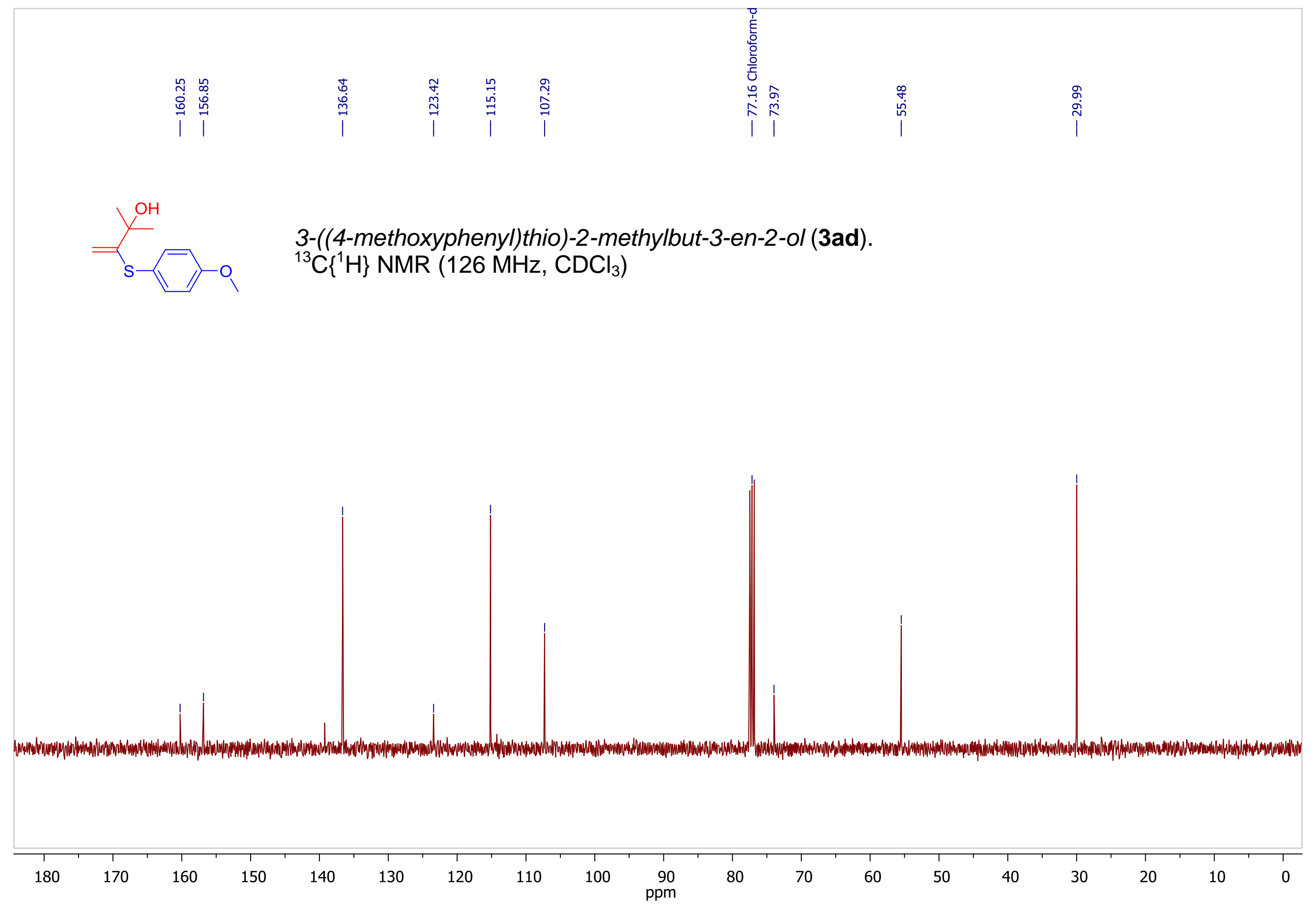




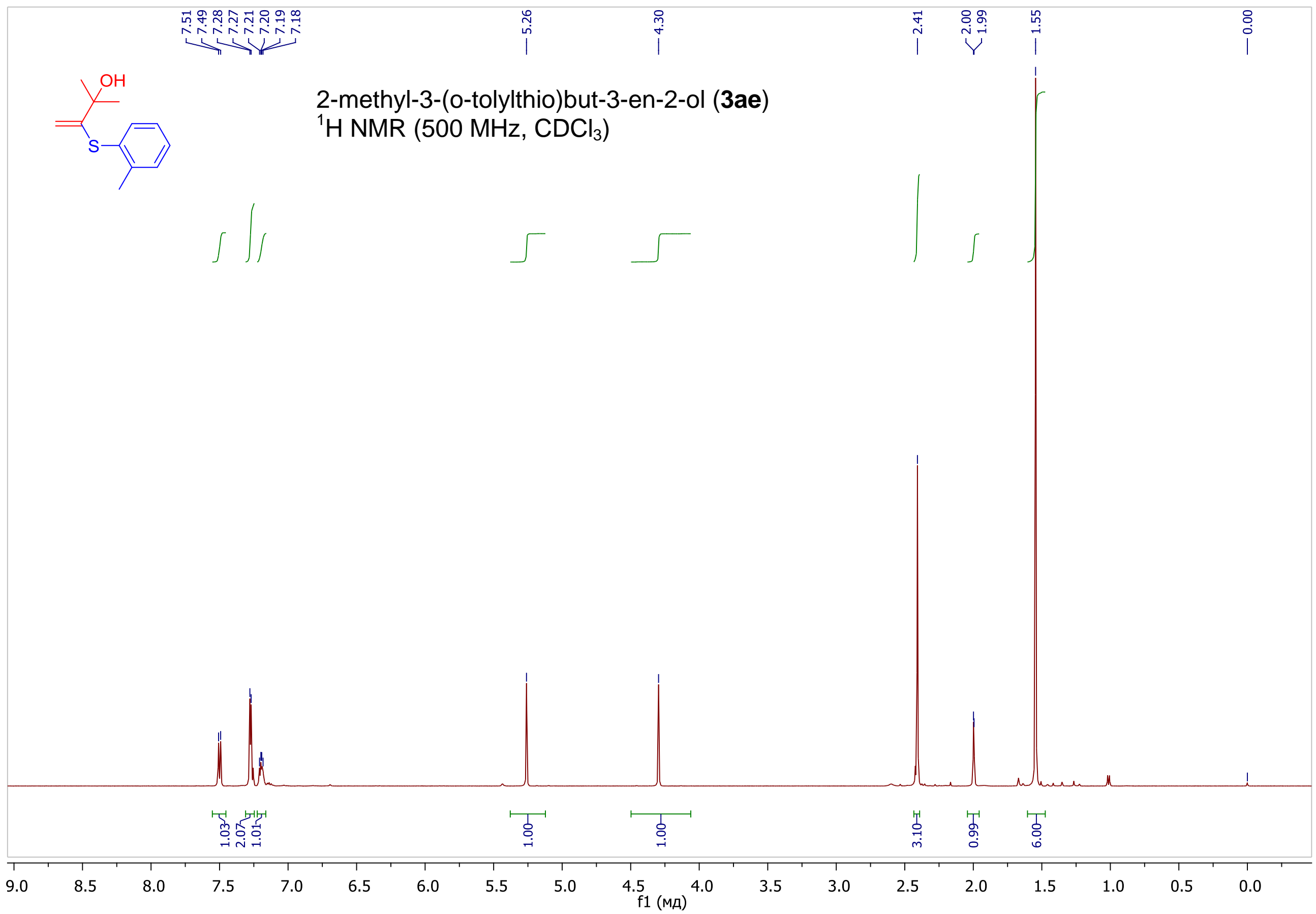




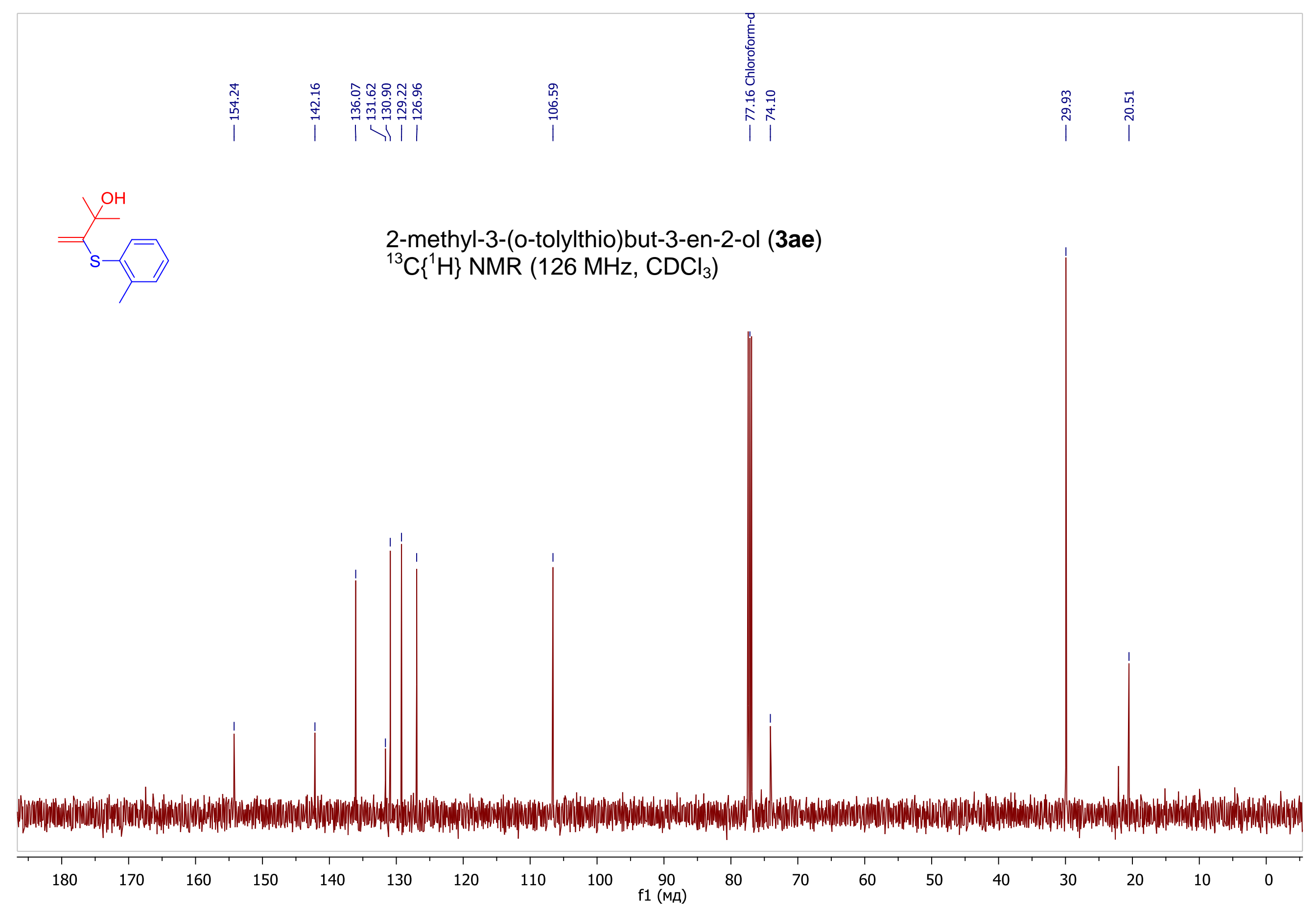




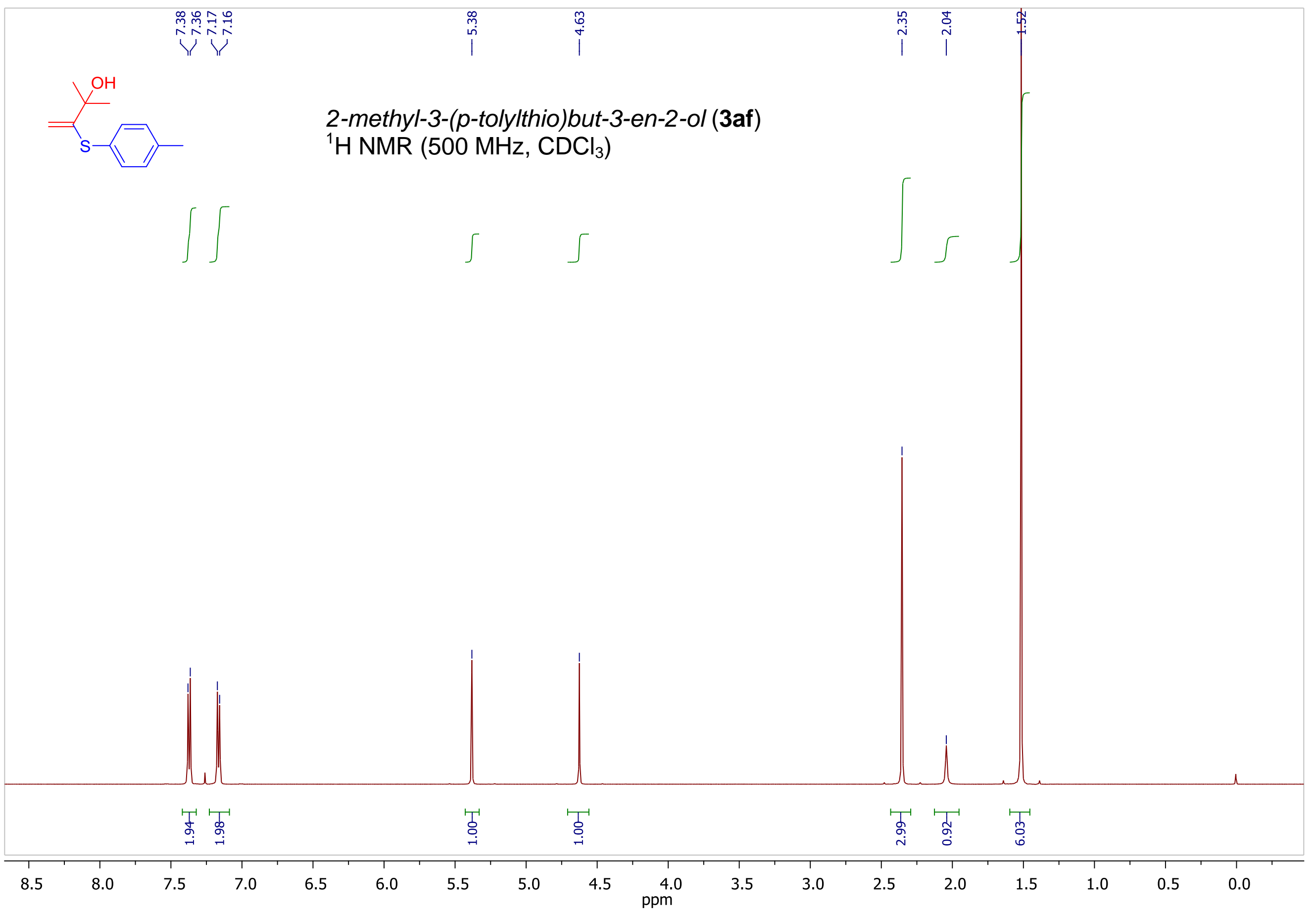




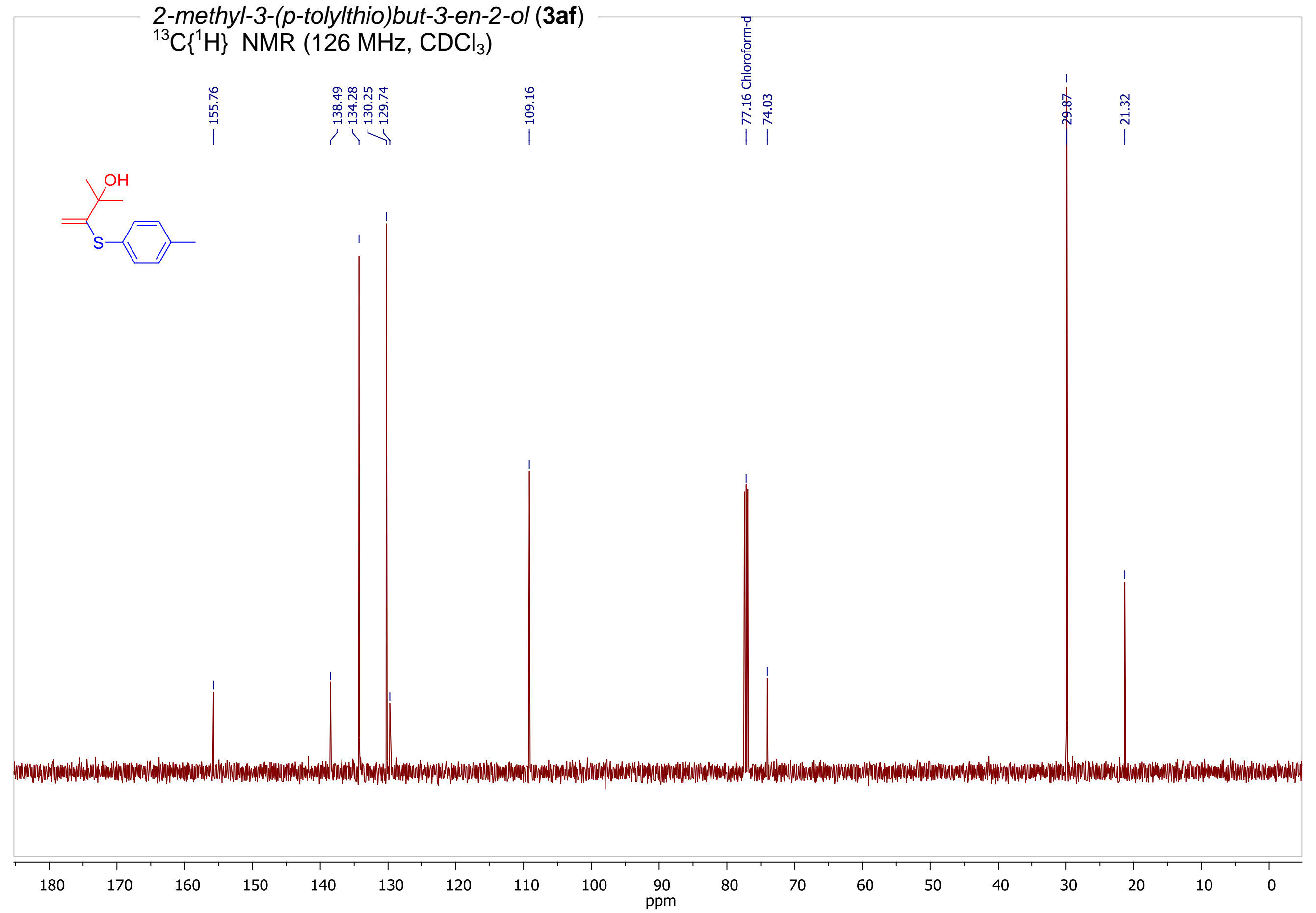




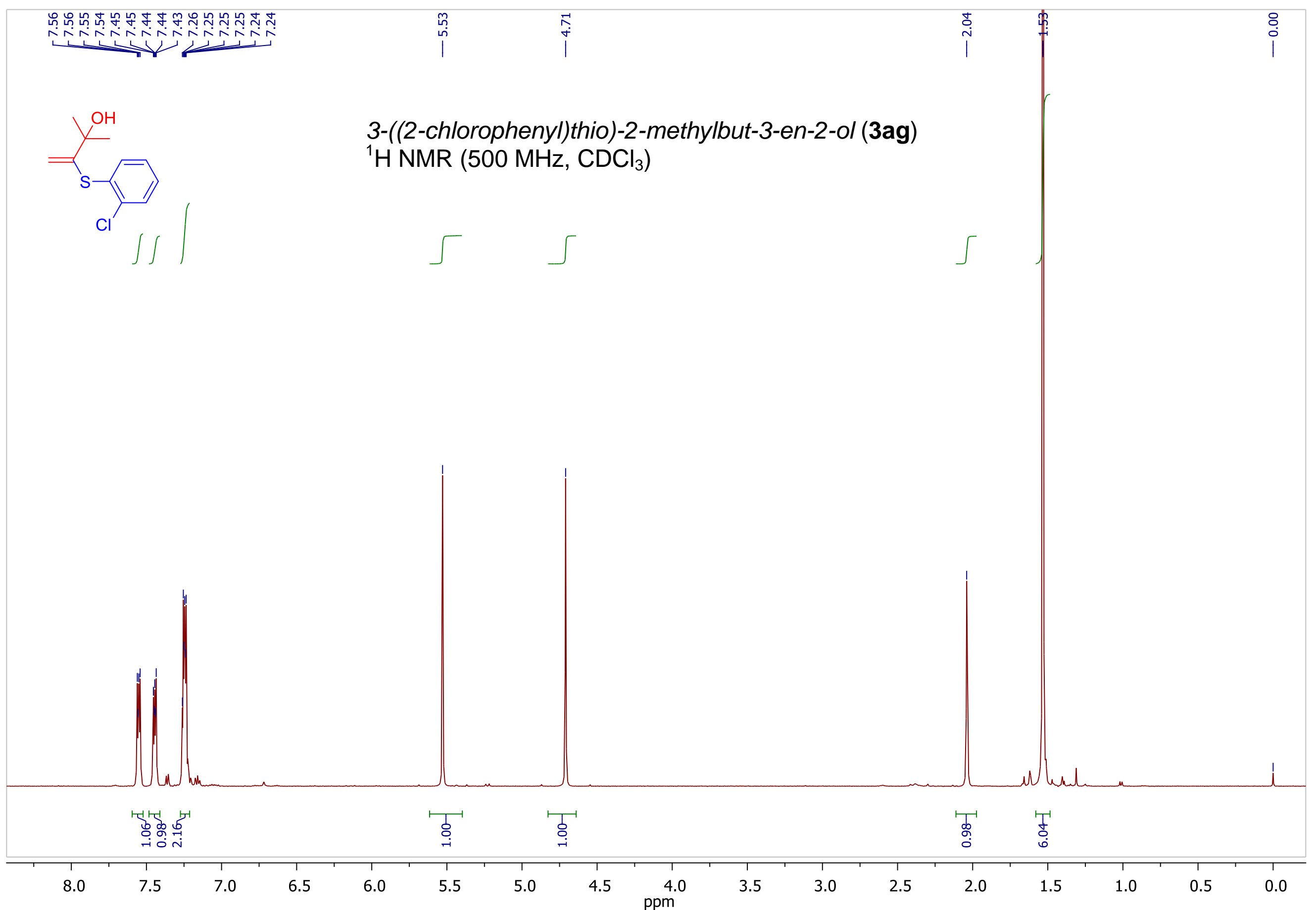




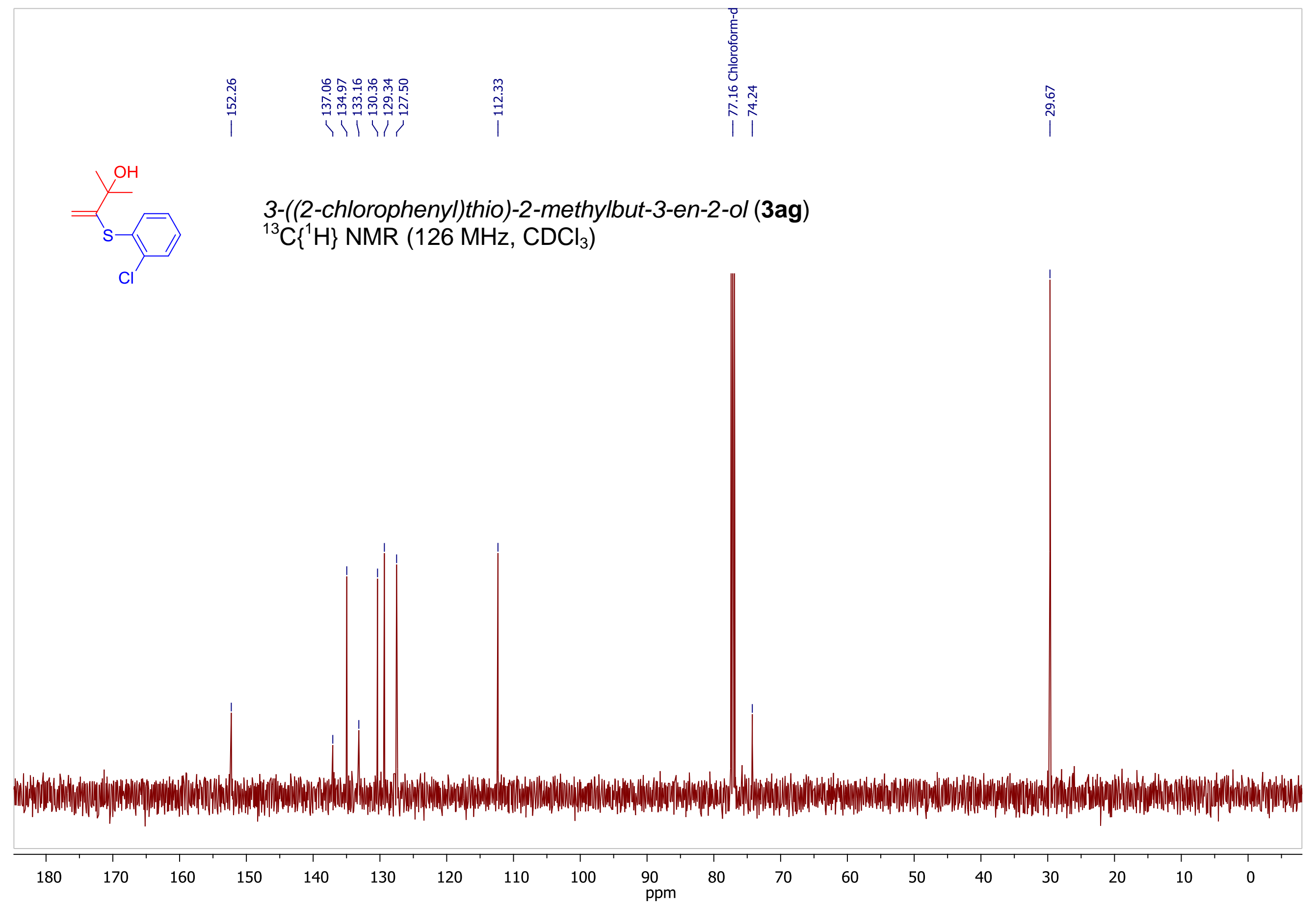




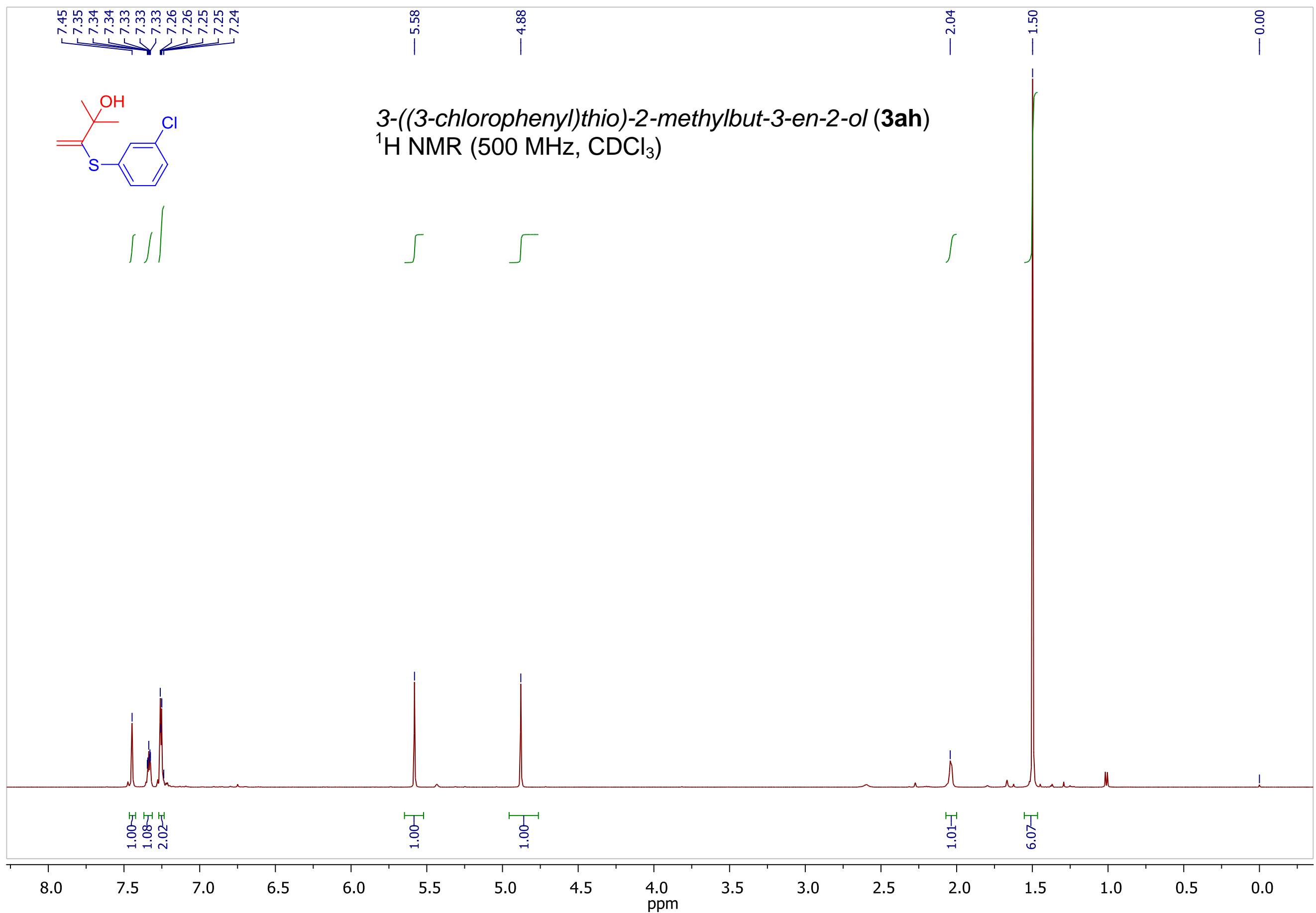




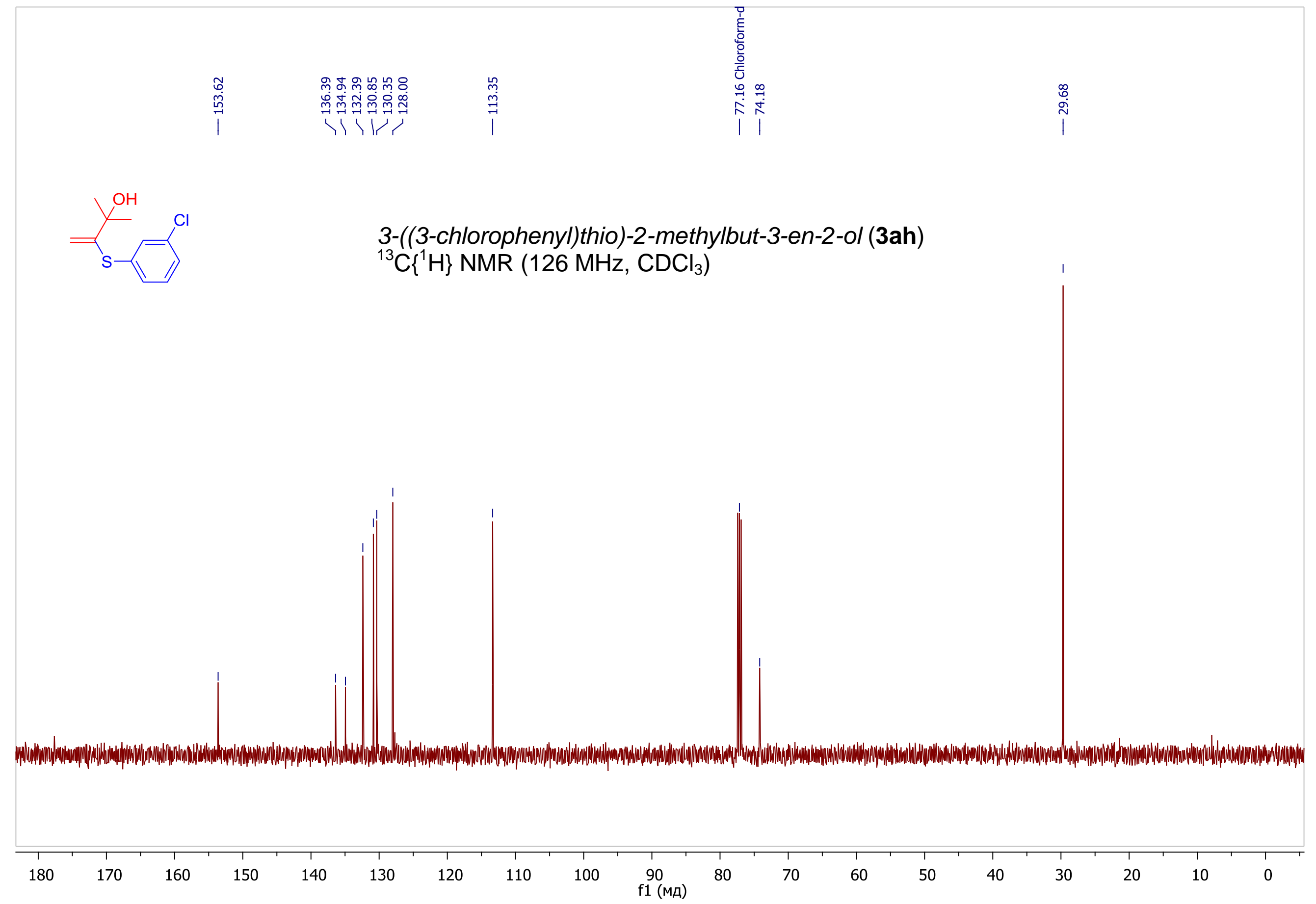


3-((4-chlorophenyl)thio)-2-methylbut-3-en-2-ol (3ai)

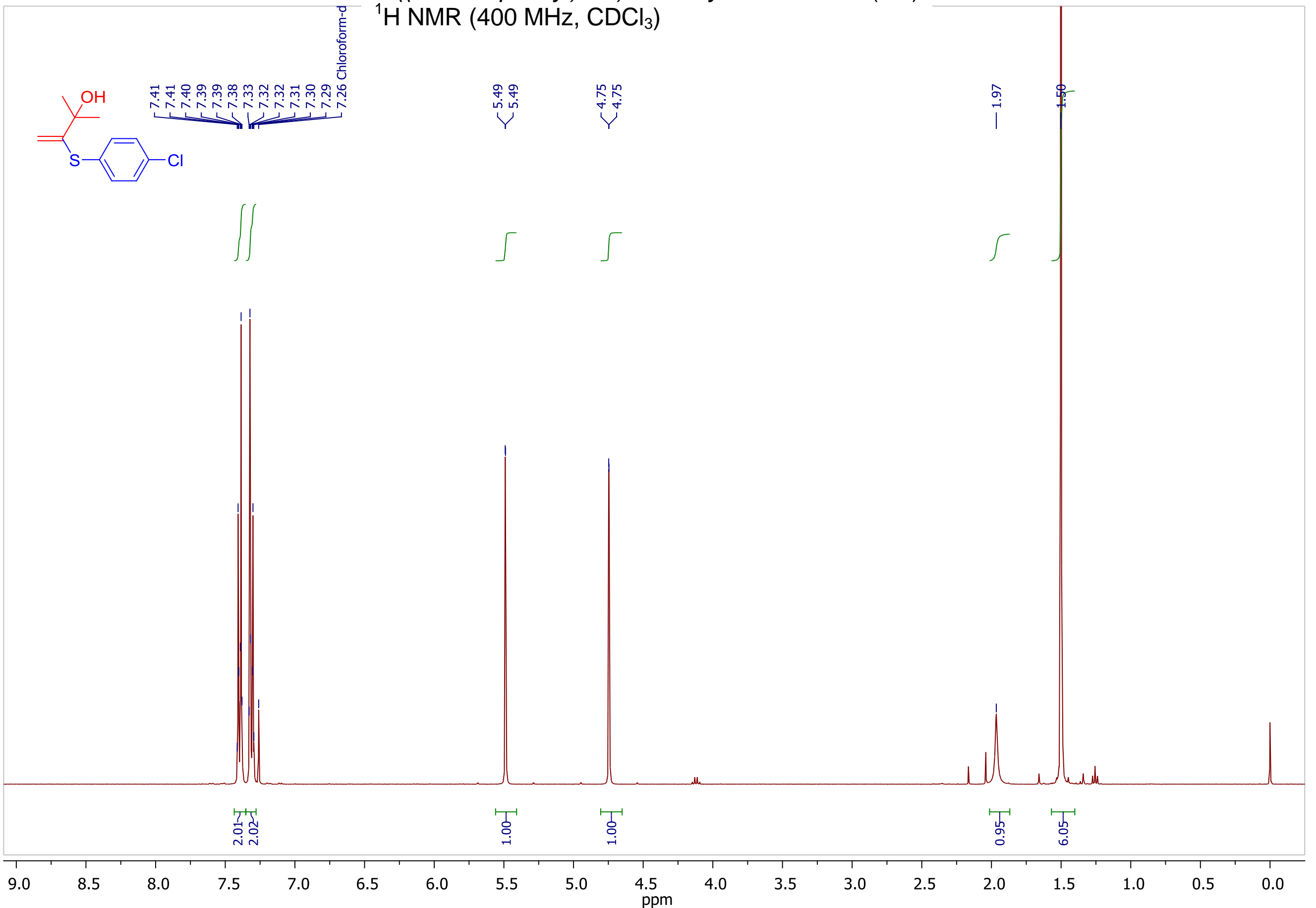




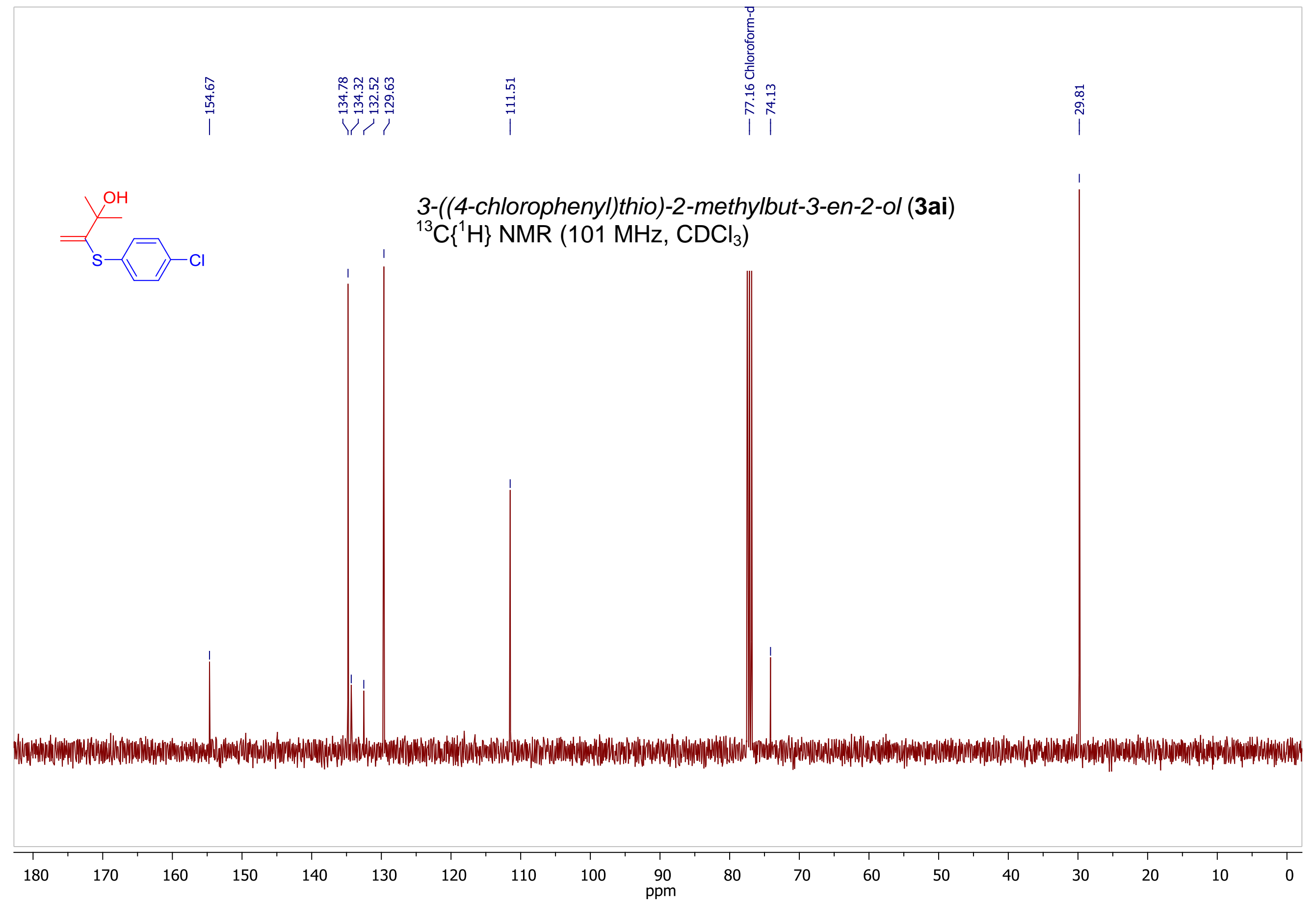




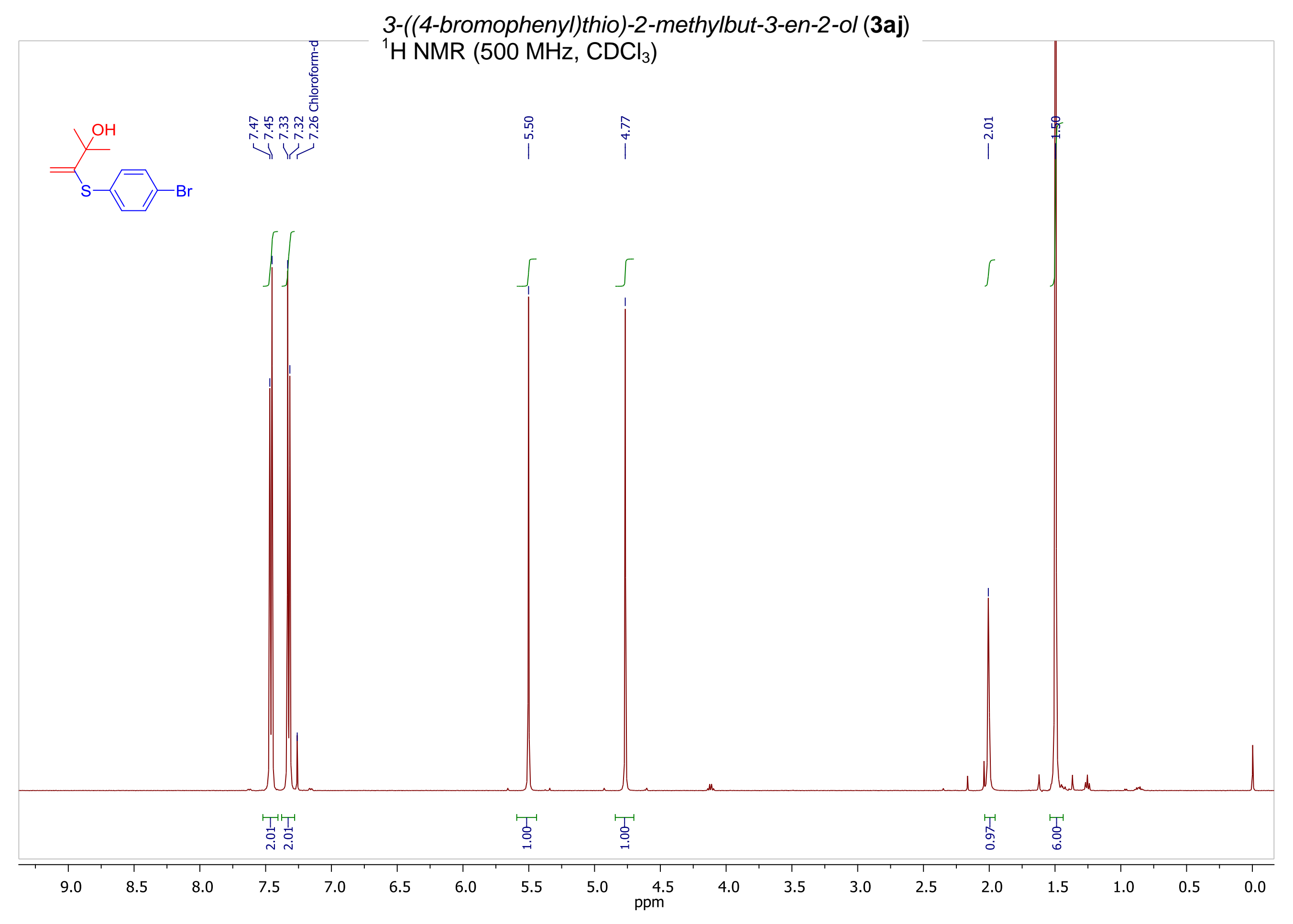




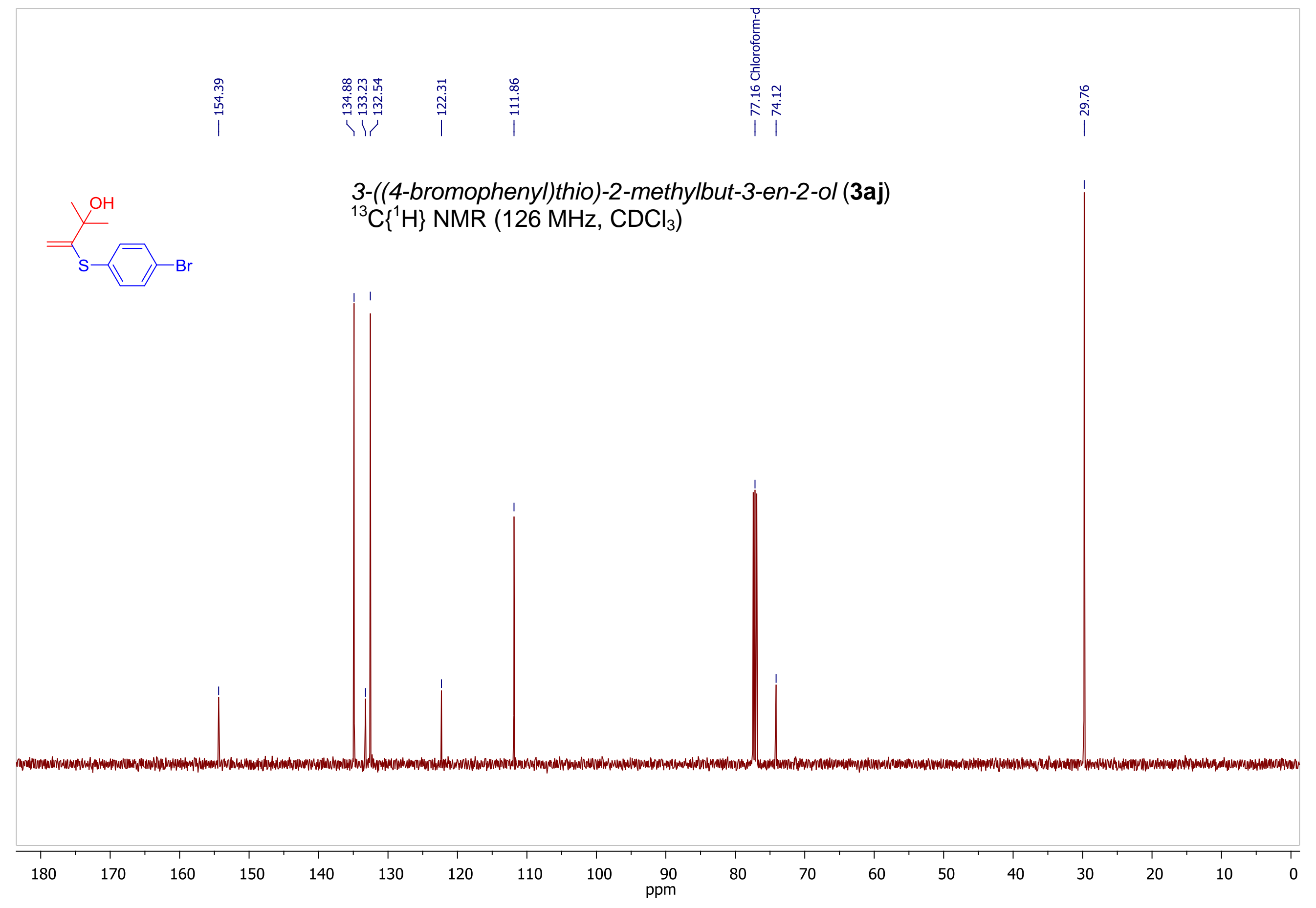




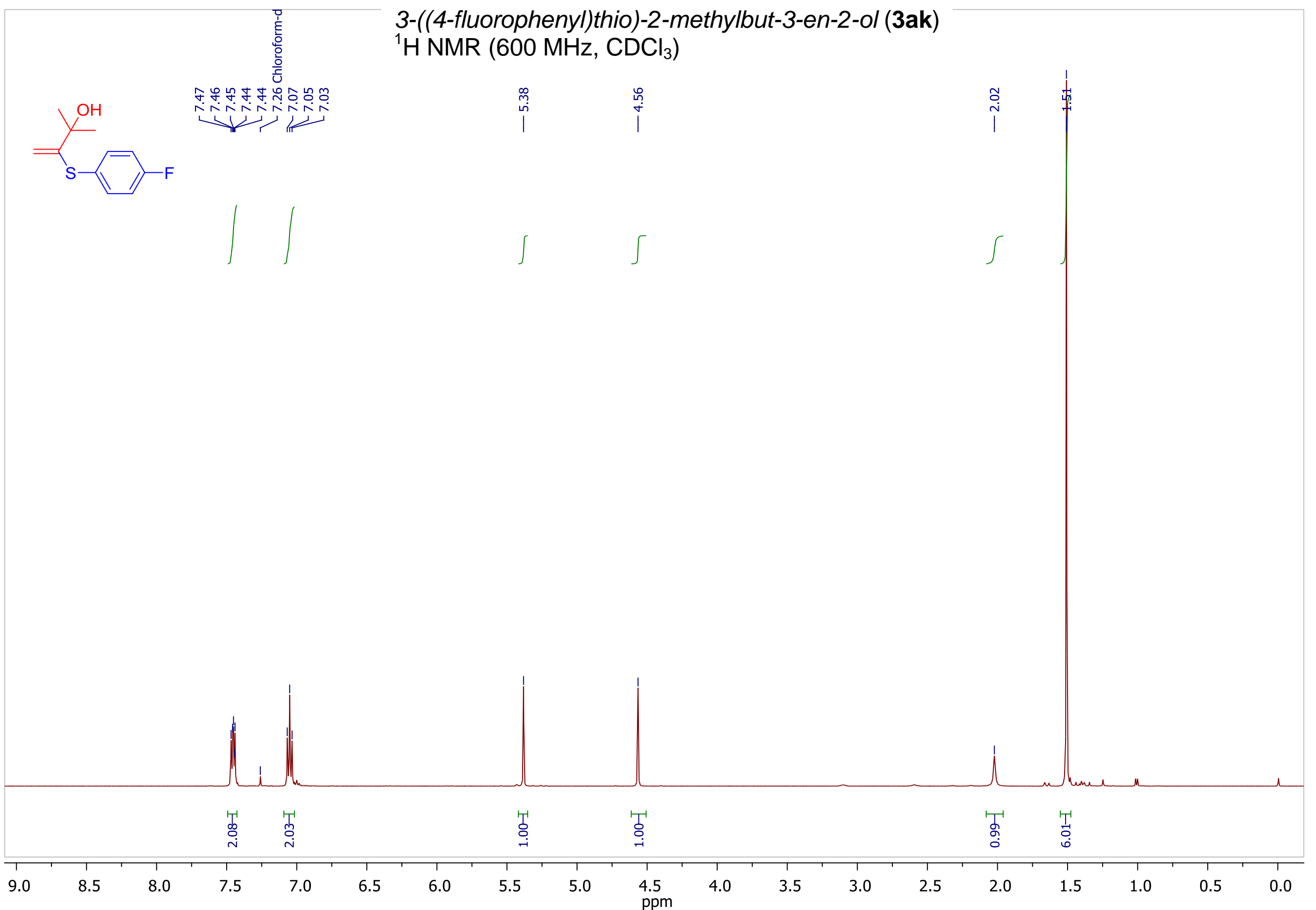




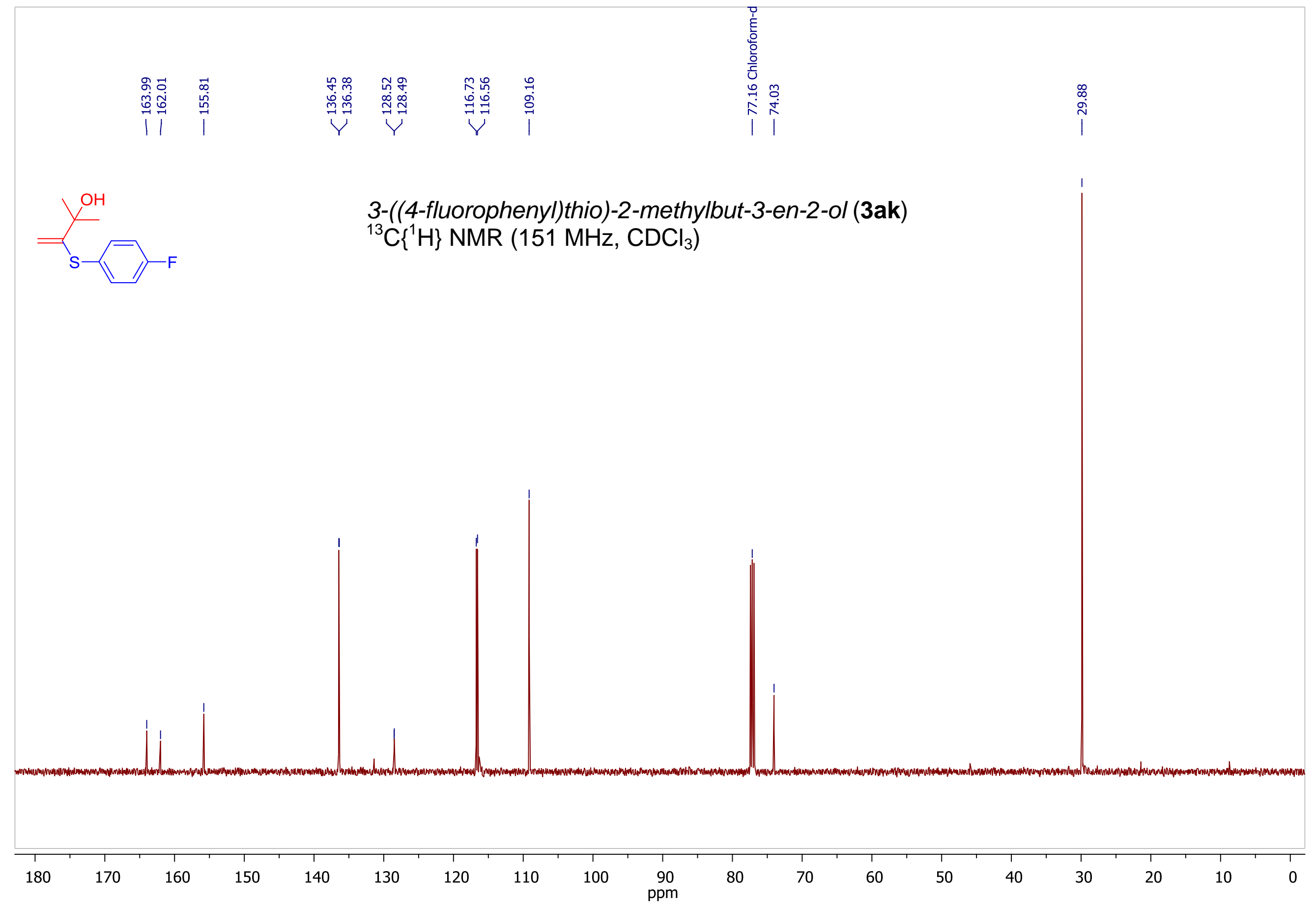




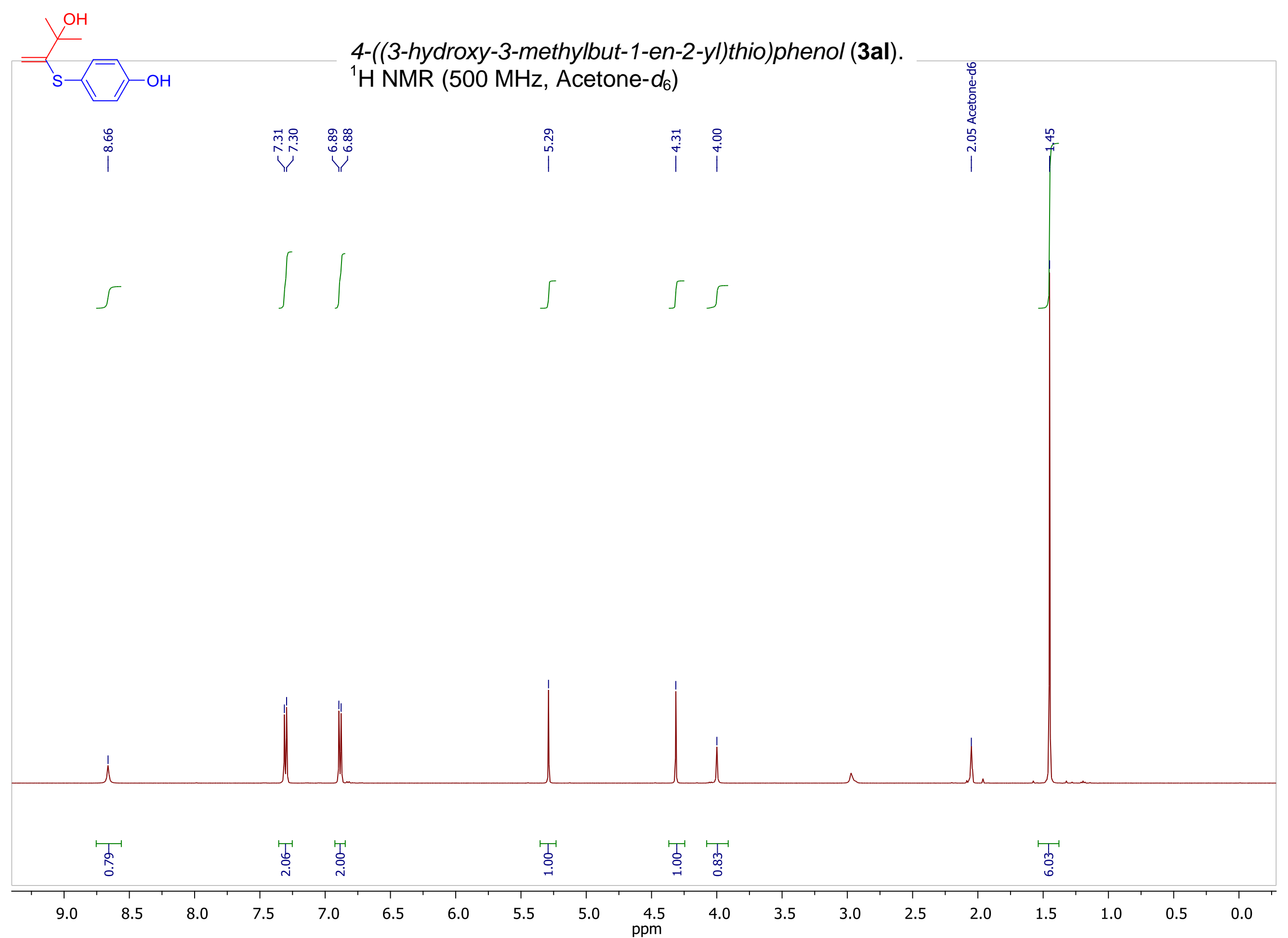




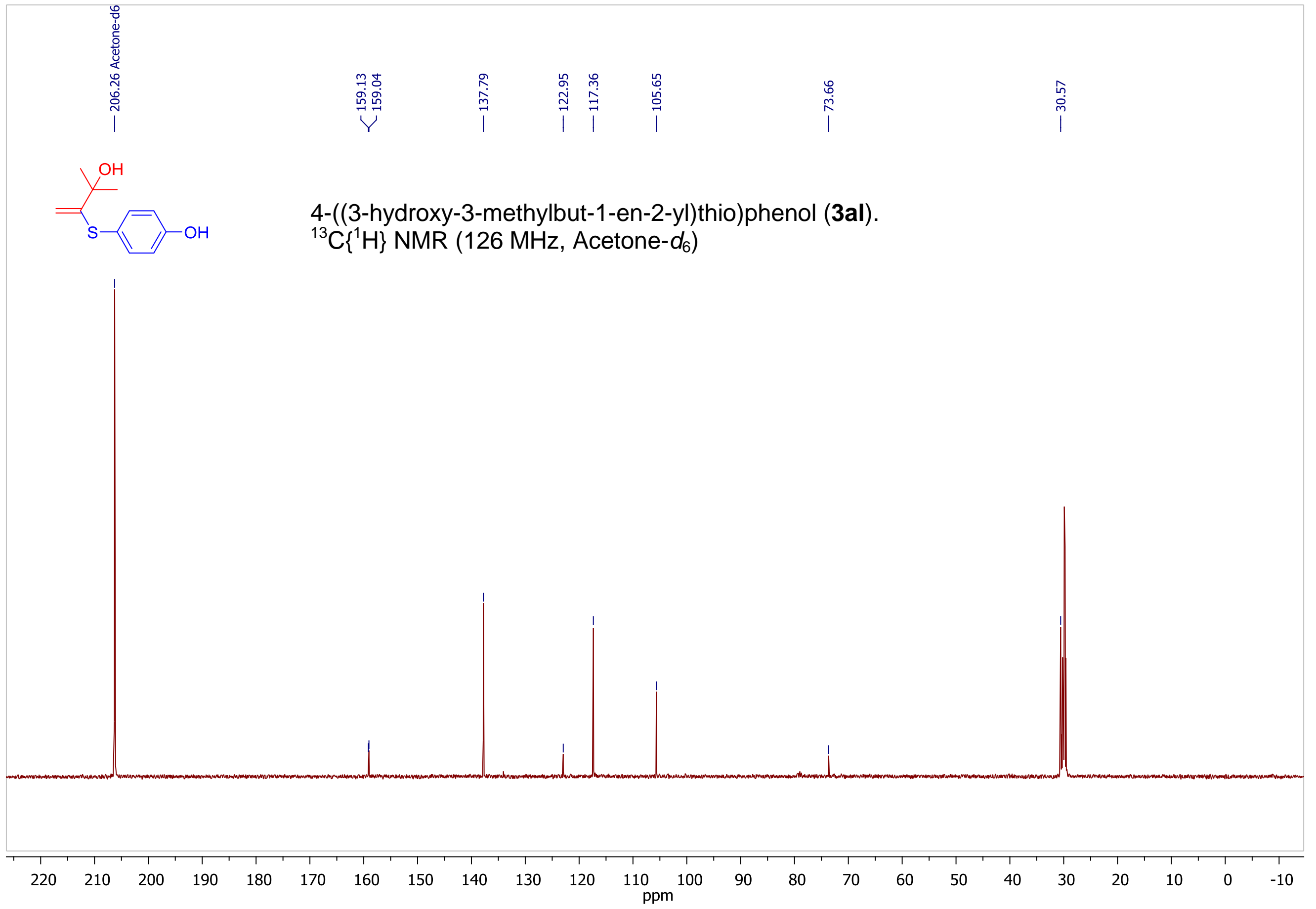




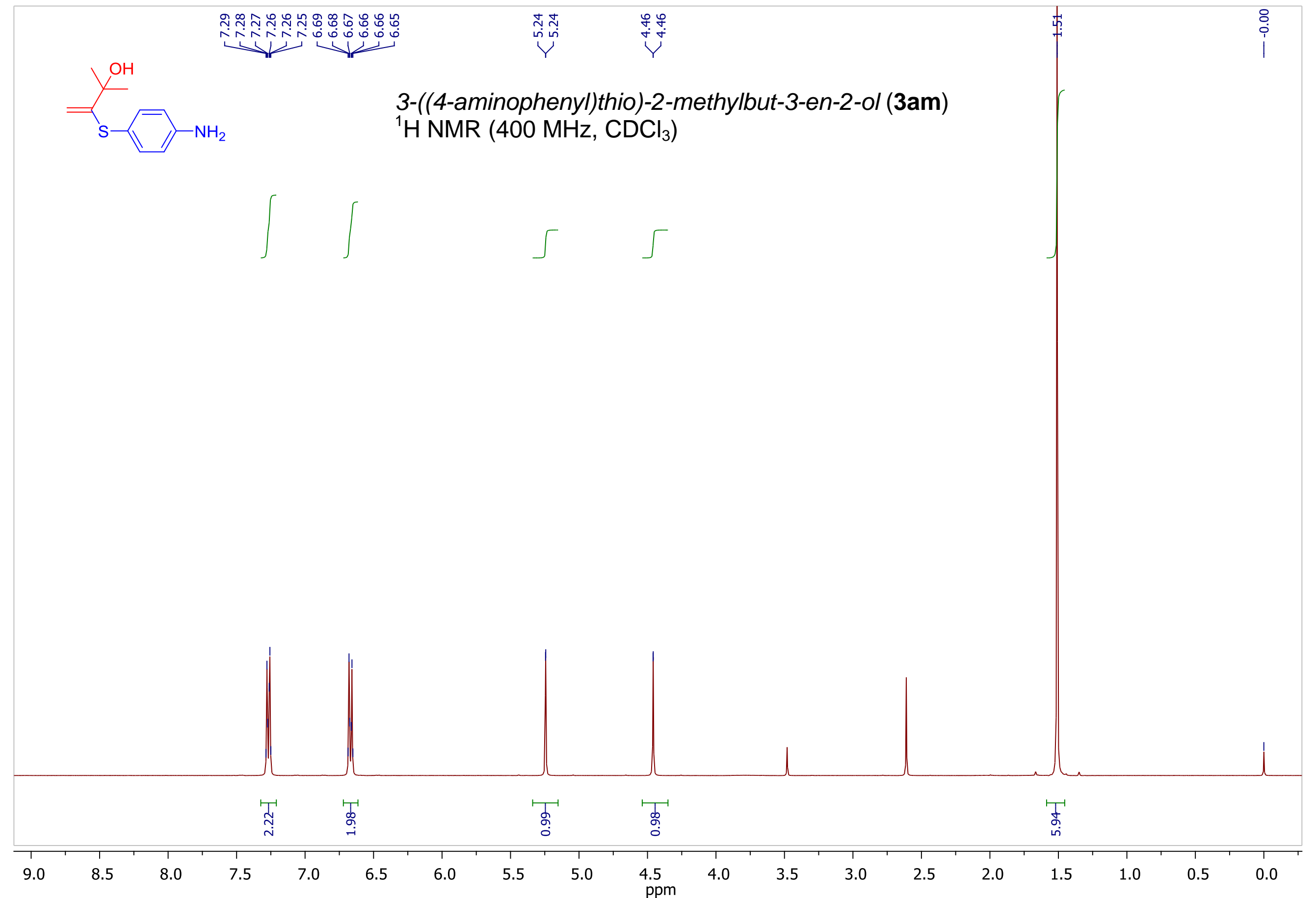




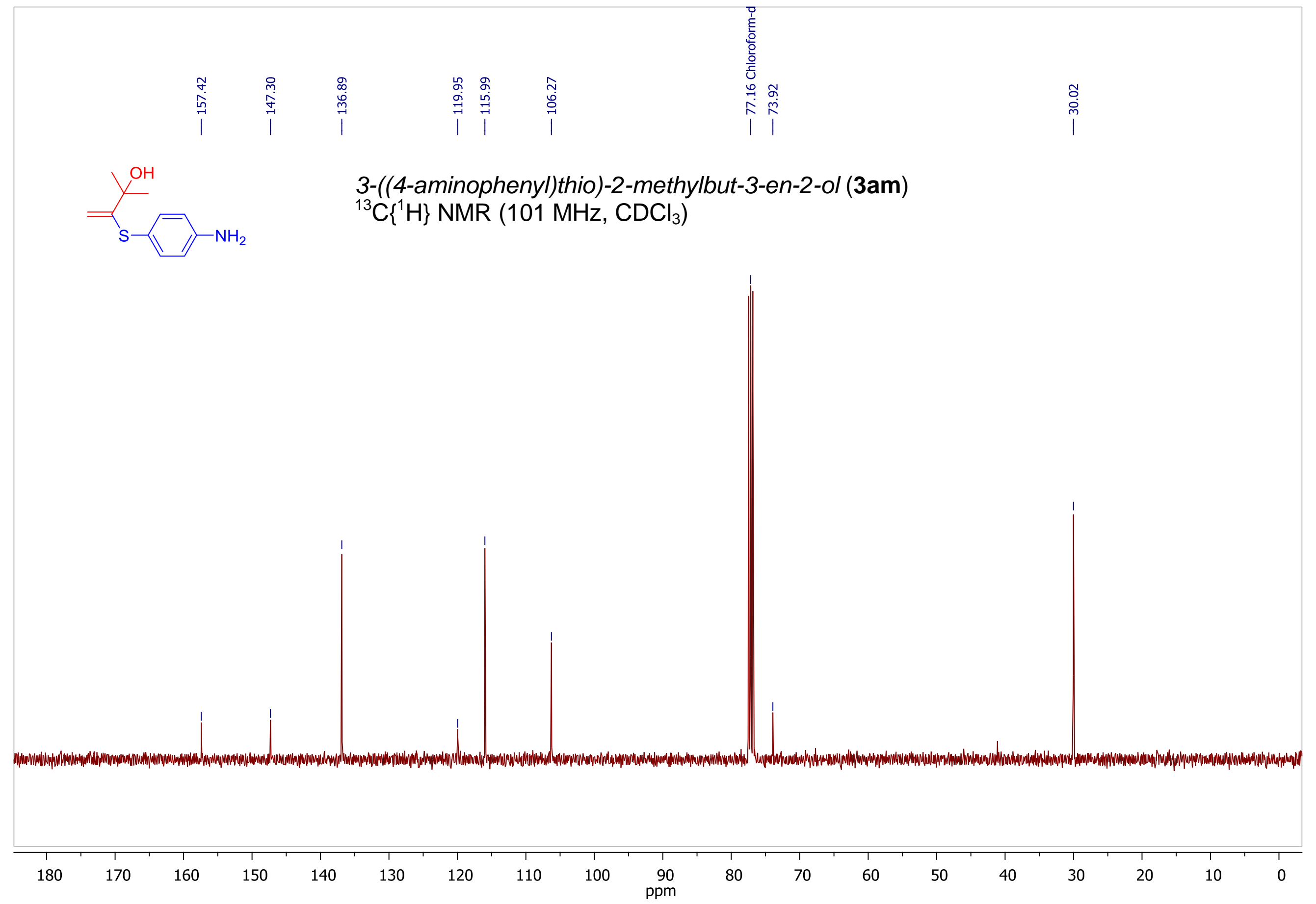




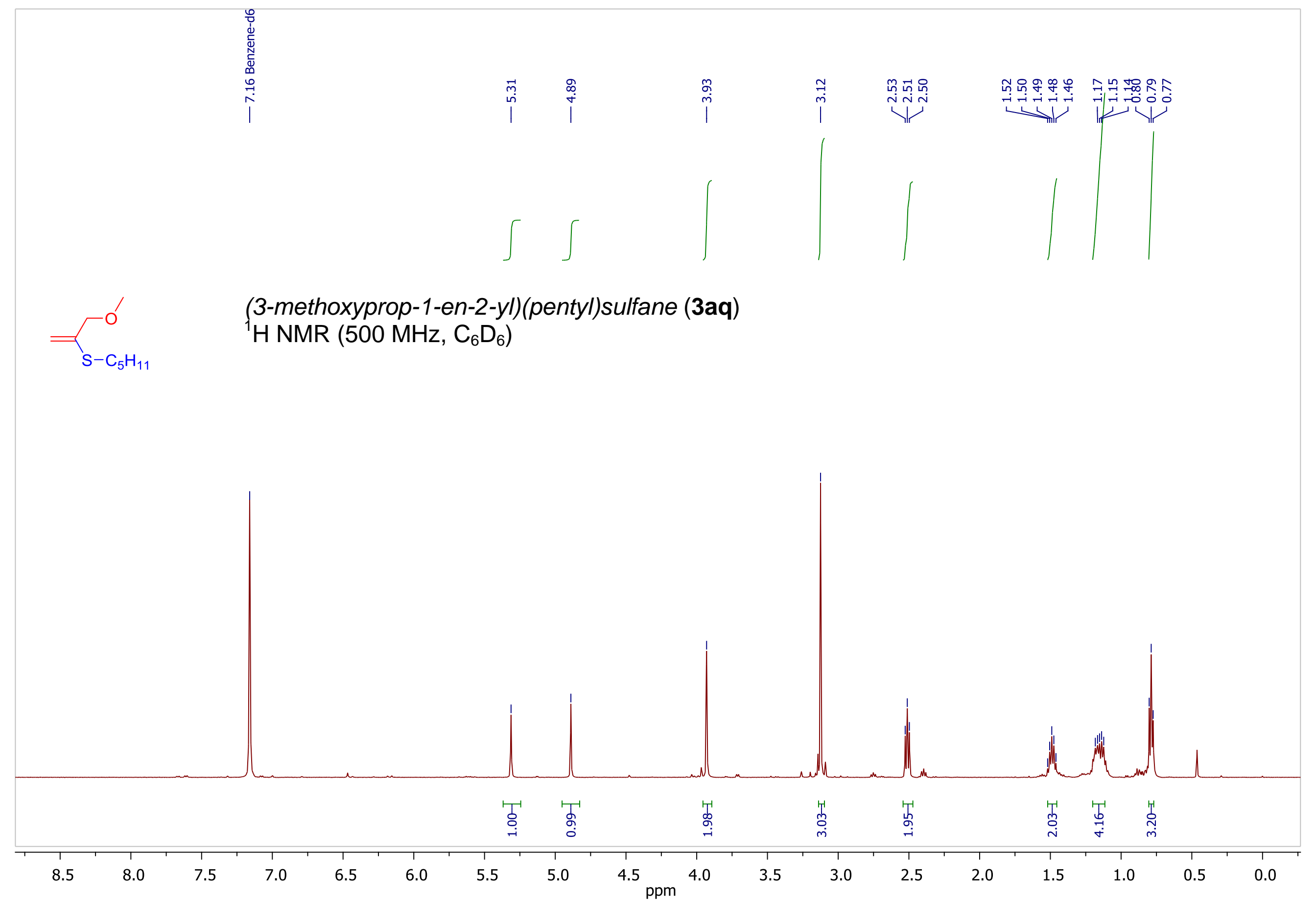




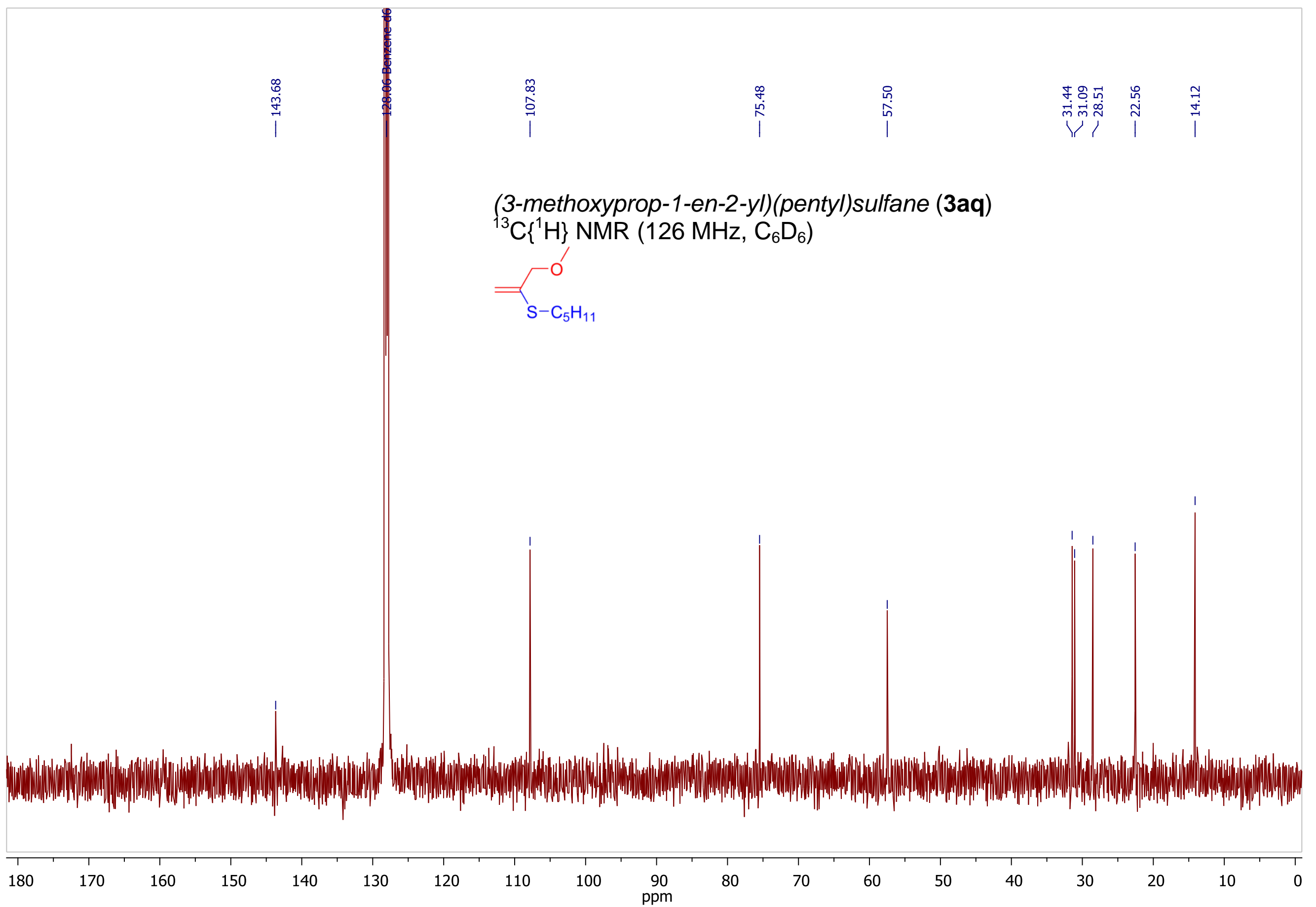




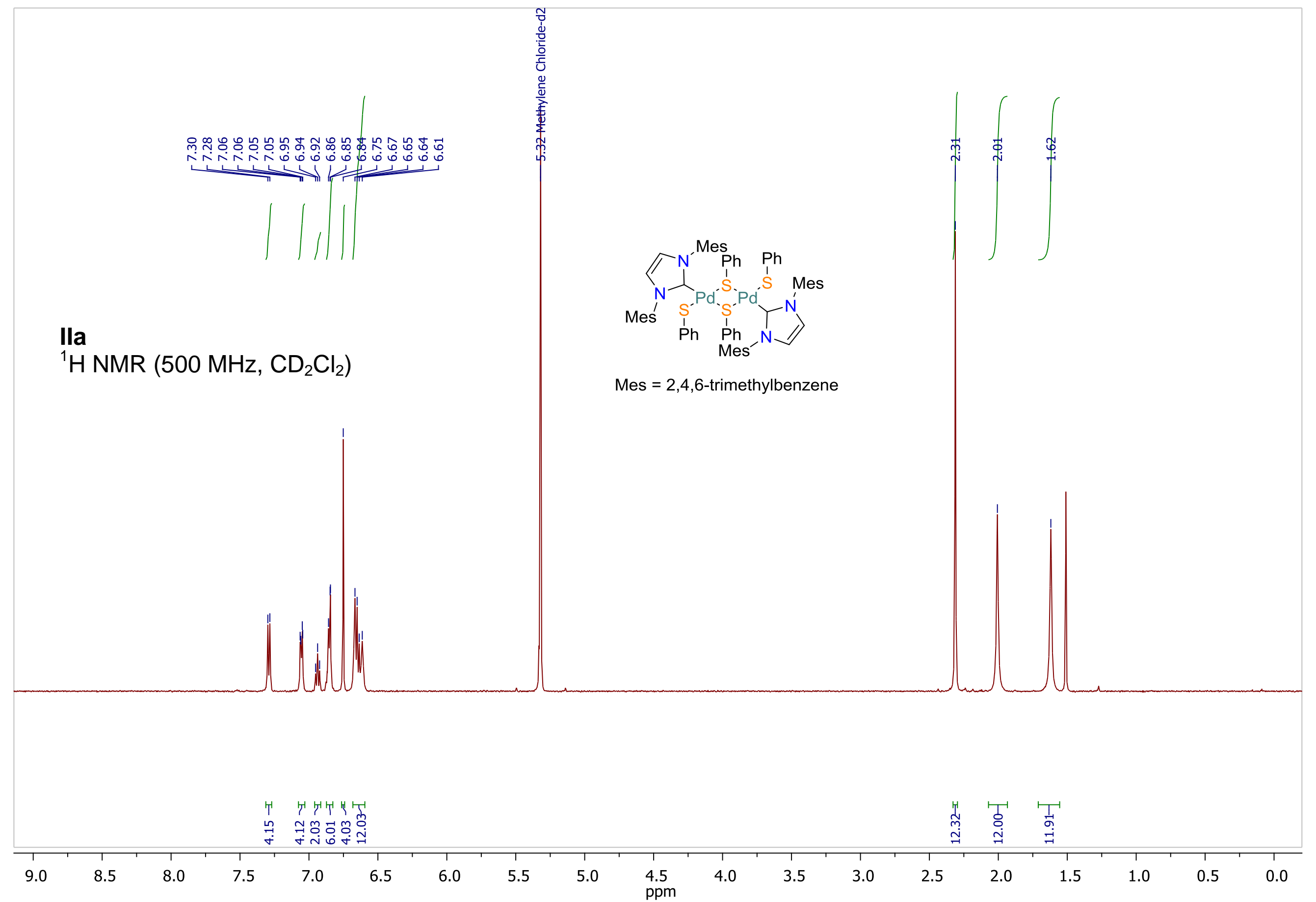



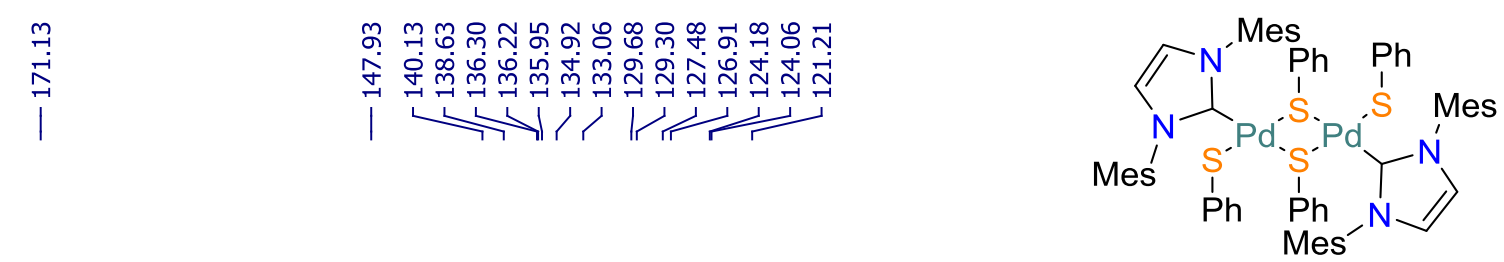

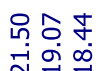

Mes $=$ 2,4,6-trimethylbenzene

Ila

${ }^{13} \mathrm{C}\left\{{ }^{1} \mathrm{H}\right\}$ NMR $\left(126 \mathrm{MHz}, \mathrm{CD}_{2} \mathrm{Cl}_{2}\right)$

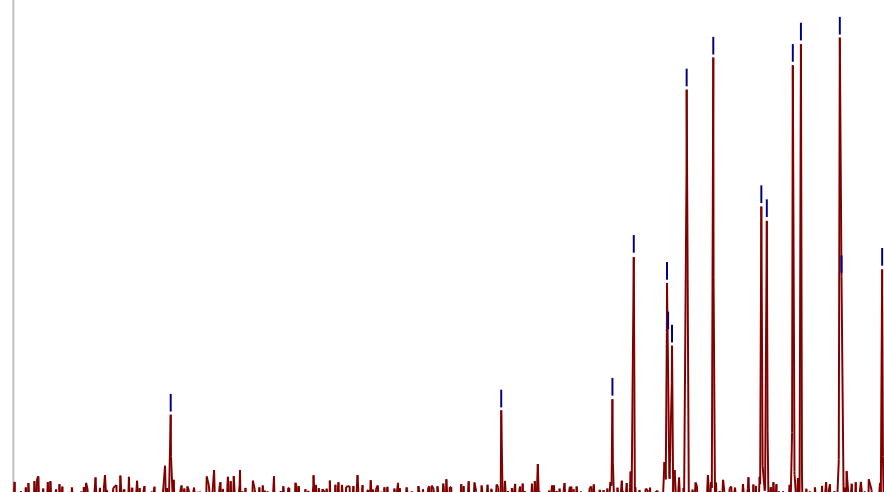

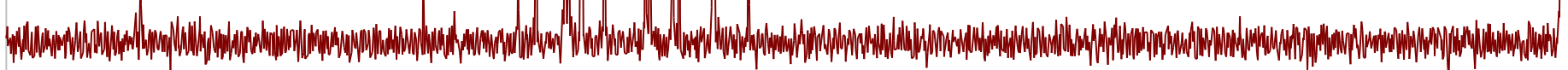

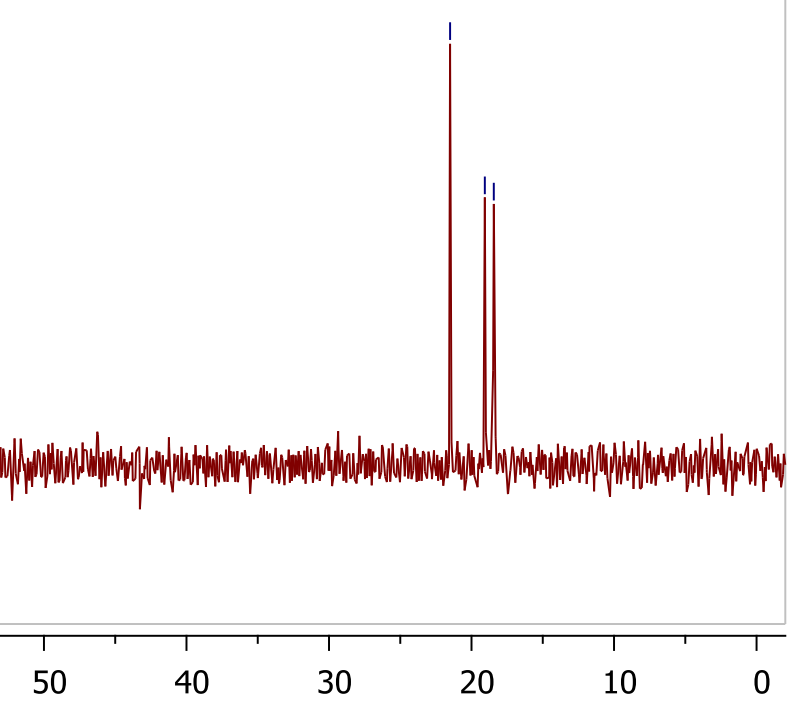

\begin{tabular}{|c|c|c|c|c|c|c|c|c|c|c|c|c|c|c|c|c|c|}
\hline \multirow{2}{*}{180} & 1 & $T$ & 1 & $T$ & $T$ & 1 & $T$ & 1 & 1 & $T$ & $T$ & $T$ & $T$ & $T$ & $T$ & $T$ & 10 \\
\hline & 170 & 160 & 150 & 140 & 130 & 120 & 110 & 100 & 90 & 80 & 70 & 60 & 50 & 40 & 30 & 20 & 10 \\
\hline
\end{tabular}

